\title{
Functional analysis of embryonic brain development in Tribolium castaneum
}

Dissertation submitted in partial fulfillment of the requirements for the degree of "doctor rerum naturalium (Dr. rer. nat.)"

of the Georg-August-University Göttingen

Nikolaus Dieter Bernhard Koniszewski

from

Erlangen,

Germany

Göttingen, 2011 
First Referee / Advisor: $\quad$ Prof. Dr. Gregor Bucher Department of Developmental Biology, Georg-August-University Göttingen

Second Referee:

Prof. Dr. Ernst Wimmer

Department of Developmental Biology, Georg-August-University Göttingen

Submission of the dissertation:

June 2011

Date of the oral examination: August 22, 2011 
Herewith I declare, that I prepared the dissertation

"Functional analysis of embryonic brain development in Tribolium castaneum" on my own and with no other sources and aids than quoted.

Göttingen, June 29, 2011

Nikolaus Koniszewski 


\section{Danksagung}

Als erstes bedanke ich mich bei Herrn Professor Bucher, der mir sein Vertrauen entgegenbrachte, so dass ich diese Arbeit selbständig durchführen durfte. Besonders danke ich ihm für die hilfreichen Diskussionen, die das schnelle voranbringen der Projekte ermöglichte. Und schließlich danke ich ihm für die häufigen Möglichkeiten, meine Ergebnisse auf internationalen Tagungen präsentieren zu dürfen. Letztlich habe ich sehr viel bei Dir gelernt, wofür ich Dir sehr dankbar bin.

Als nächstes und nicht weniger Dank gebührt Herrn Professor Wimmer, der durch seine kritischen und hilfreichen Fragen einen erheblichen Einfluss auf den Verlauf und den Ausgang dieser Arbeit nahm. Auch dafür möchte ich mich bei ihm bedanken, dass er immer ein offenes Ohr und stets Ideen parat hatte um mögliche methodischen Probleme auszumerzen.

Herrn Professor Averof und Hendrikje Hein danke ich für die mir zur Verfügung gestellten Linien. Außerdem möchte ich Martin Kollmann und Herrn Professor Joachim Schachtner für die unkomplizierte Unterstützung und Kooperation danken. In diesem Kontext möchte ich auch Stefan danken.

Recht herzlich möchte ich allen Mitarbeitern des Instituts Entwicklungsbiologie danken, die mir alle immer hilfsbereit mit Rat und Tat zur Seite standen, wenn es nötig war. Besonderer Dank gilt Elke, die es immer wieder schaffte tot geglaubte Käfer wieder zu beleben, und Beate, die immer wieder versuchte mir die Welt der Computer näher zu bringen. Auch danke ich Birgit, Angelika, Helma, Barbara, Kathrin und Sabine, die immer zu ein kleinen netten Gespräch aufgelegt waren (oder auch zu andere Scherzchen), und damit ein wunderbare Arbeitsatmosphäre schafften.

Ein weiterer Dank gilt allen Bewohnern des Labor 3, auch jenen, die nur kurz vorbeikamen. Sie alle haben zu einem lebhaften, lustigen und turbulenten Arbeitsalltag beigetragen. Dabei möchte ich besonders Matthias, Georg, Inga, Sebastian und auch Monique danken.

Schließlich danke ich Jonas und Chris, die mich immer gut sicherten, wenn es darauf ankam.

Außerdem danke ich meinen Eltern und meiner Familie, die mich immer unterstützt haben und ohne die ich dies alles niemals erreicht hätte.

Aber der größte Dank gehört meiner Frau Sabine, einfach weil Du immer für mich da bist! Vielen Dank! 
List of Tables 8

List of Figures .8

Summary .10

\section{Introduction.} .12

1.1 Conservation of the bilaterian brain .12

1.2. From neuroectoderm to neuropile. .14

1.2.1. Specification of neuroblast identity .16

1.2.2. Neuroblast lineages...

1.3. The insect brain.

1.4. Tribolium castaneum as a model organism for studies of brain development 21

1.5. The larval brain of Tribolium castaneum. .22

1.6. Candidate genes for NB identity specification in the insect brain. .24

1.7. Aims of the study. .26

2. Material and Methods .27

2.1. Tribolium castaneum strains used as source of genomic DNA and for embryonic transgenesis 27

2.2. Amplification of the 5'UTR by RACE-PCR .27

2.3. Amplification and cloning of regulatory regions .28

2.4. Whole mount in situ hybridization .29

2.5. Embryonic and pupal knock down of gene function by RNAi...

2.6. Embryonic transgenesis .31

2.7. Larval brain in vivo imaging...

2.8. Immunohistochemistry on larval brains .33

2.9. Heat shock in water bath. 34

2.10. Documentation 34 
2.11. Used computer programs 34

3. Results and Discussion Part I: Generation of transgenic reporter lines for the analysis of brain development in Tribolium castaneum .....................................................................................35

3.1. The heat shock Tc-six 3 overexpression construct is not functional............................................35

3.1.1. Characterization of positive transgenic animals by in situ hybridization and cuticle phenotype.

3.1.2. Genomic DNA analysis reveals errors in construct sequence 37

3.2. Generation of reporter lines regulated by the native upstream regions of Tc-elav and Tc-repo..38

3.2.1. The transgenic line for the regulatory region of Tc-elav revealed a muscle expression pattern

3.2.2. The transgenic line for the regulatory region of Tc-repo shows a glial expression pattern 40

3.3. Generation of Tc-six 3 and Tc-rx reporter lines

3.3.1. Identification of Tc-six 3 and Tc-rx positive NBs in the anterior neuroectoderm

3.3.2. Tc-six 3 reporter lines

3.3.2.1. Generation of Tc-six 3 reporter lines 47

3.3.2.2. Analysis of transgenic animals carrying for the Tc-six 3 reporter line construct

3.3.3. Tc-rx reporter lines .59

3.3.3.1. Generation of the Tc-rx reporter lines

3.3.3.2. Analysis of transgenic animals carrying the Tc-rx reporter line constructs

3.3.5. Verification of transgenic reporter lines by PCR and schematic summary of the characterization of the transgenic animals.

3.4. Analysis of the fluorescence signal of the 'Mushroom Body' line and the 'Brainy' line .72

4. Results Part II: The genetic network of Central Body development .77

4.1. Tc-rx knock down leads to a split Central Body .77

4.2. Tc-otd1 is not the antagonist of Tc-six3 in Central Body growth...... .78

4.3. Tc-six 3 and Tc-chx are required for Central Body formation. .81 
4.3.1 Tc-chx and Tc-six3 coexpression analysis. .82

4.3.2 At least 4 NBs delaminate from the Tc-chx positive anterior neuroectoderm. .85

4.3.3 pRNAi of Tc-six3 and Tc-chx in brain imaging lines led to loss of the Central Body..... .87

4.3.4 Immunohistochemistry analysis of the brain phenotypes resulting from Tc-six 3 and Tc-chx knock down. .90

4.3.5 Temporal separation of the epidermal and neural functions of Tc-six3.

5. Discussion .97

5.1. Reporter systems in Tribolium castaneum......

5.1.1 Improving the Tc-repo and Tc-elav reporter lines

5.1.2. Low efficiency of the Tc-six 3 reporter constructs .98

5.2. The 'Tc-rx 5up' line for studies of axon guidance and brain midline cues

5.3. The 'Brainy' reporter line allows analyses of embryonic brain development regarding the function of Tribolium homologues to vertebrate neural plate genes

5.4. Results regarding the embryonic brain formation in Tribolium castaneum.....

5.4.1. Tc-otd1 has a conserved function in specifying the anterior part of the brain. 103

5.4.2. The role of Tc-rx in Central Body formation. 105

5.4.3. Tc-six 3 and Tc-chx are required for Central Body formation. 107

5.4.4. Personal assumption of a hierarchical function of the studied genes in the context of Central Body development in Tribolium castaneum

5.6. Outlook 116

6. References

7. Appendix 135

8. Abbreviations 156

9. Curriculum vitae 157 


\section{List of Tables}

Table 1: Primers used for 5'UTR amplification

Table 2: Primers used for the amplification of regulatory regions including restriction sites

Table 3: Primers for the amplification of reporter genes

Table 4: Sequencing primers of the pre-reporter gene regions

Table 5: Antisense probes of genes used for in situ hybridization

Table 6: Embryonic (emb.) and pupal (pup.) RNAi of different genes

Table 7: Injected constructs and their positive selection marker

\section{List of Figures}

Figure 1: Combinations of marker gene expression reflect individual identities of brain NBs

Figure 2: Scheme of an adult insect brain

Figure 3: The larval brain of Tribolium castaneum

Figure 4: In situ hybridization for Tc-six3 in the Tc-six3 overexpression line after heat shock for 10 min at $46^{\circ} \mathrm{C} \quad 36$

Figure 5: Sequence analysis of the linker between the hsp5 -UTR and the $T c$-six3 ORF reveals additional translation start sites

Figure 6: The line 'reg. Tc-elav::mRFP' drives reporter gene expression in muscles

Figure 7: The reporter gene expression pattern of the line 'reg. Tc-repo::tGFP' mimics the endogenous Tc-repo expression in WT-embryos with delay

Figure 8: Expression analysis of tGFP in L1 larva of the line 'reg. Tc-repo::tGFP' 42

Figure 9: At least 8 NBs delaminate from the Tc-six3 positive region 44

Figure 10: At least 9 NBs delaminate from the Tc-rx positive region 45

Figure 11: 5 'UTR and cloning strategy for the transgenic reporter lines of Tc-six3 48

Figure 12: In situ hybridization with an antisense probe for tGFP in the transgenic line 6 containing the construct ${ }^{`} T c$ six3 10up'

Figure 13: In situ hybridization with an antisense probe for tGFP in the transgenic line 7 containing the construct ${ }^{`} T c$ six3 10up' 52

Figure 14: tGFP expression analysis in larvae of the transgenic line 7 carrying the construct 'Tc-six3 10up' 54 Figure 15: In situ hybridization with an antisense probe for tGFP in the transgenic line 9 containing the construct ${ }^{`} T c$ $\operatorname{six} 3$ Sup' $^{\prime}$

Figure 16: In situ hybridization with an antisense probe for tGFP in the transgenic line 10 containing the construct `Tcsix3 5 up $^{\prime}$

Figure 17: In situ hybridization with an antisense probe for tGFP in the transgenic line 3 containing the construct ' $T c$ six3 5down'

Figure 18: The 5'UTR and the cloning strategy for the transgenic reporter lines of $T c-r x$

Figure 19: In situ hybridization with an antisense probe for DsRedEx in the transgenic line 1 containing the construct

'Tc-rx 5up'

Figure 20: Analysis of reporter gene expression in the brain of a larva transgenic for 'Tc-rx 5up'

Figure 21: In situ hybridization with an antisense probe for DsRedEx in the transgenic line 11 containing the construct 'Tc-rx Intron'

Figure 22: Analysis of reporter gene expression in the brain of a transgenic larva for 'Tc-rx Intron'

Figure 23: PCR analysis of $T c-r x$ and $T c-s i x 3$ reporter lines by screening gDNA for reporter gene insertion

Figure 24: Summary of the analyses of transgenic reporter lines 
Figure 25: Characterization of the Mushroom Body (MB) line by crossing with the glial marker line 6xP3::DsRedEx

Figure 26: Embryonic developmental series of the fluorescence signal in the 'Brainy' (BA) line 75

Figure 27: Knock down of Tc-rx leads to a split Central Body in first larval instar brains 78

Figure 28: Knock down of Tc-otdl leads to a reduction of brain size 79

Figure 29: Correlation between the point in time of Tc-otd1 RNAi and brain phenotypes - early knock down leads to strong phenotypes 80

Figure 30: Correlation between head capsule and brain size after Tc-otdl knock down 81

Figure 31: Double in situ hybridization of $T c-c h x$ and $T c$-six3 demonstrates an overlapping expression pattern in up to $24 \mathrm{~h}$ old embryos

Figure 32: Tc-chx expression pattern 84

Figure 33: The number of NBs positive for Tc-chx varies in similarly aged embryos 86

Figure 34: Tc-six3 cuticle RNAi phenotypes 87

Figure 35: Tc-six3 and Tc-chx knock down leads to a loss of the Central Body 88

Figure 36: Tc-six3 and Tc-chx knock down leads to loss or cleavage of the Central Body (CB) 91

Figure 37:The epidermal and neural function of Tc-six3 is not separable by late embryonic RNAi 94 


\section{Summary}

The brain is one of the most complex organs in animals. It allows the coordination of complex operations like orientation and directed movements. In insects, a structure in the brain has been identified that represents the superordinate center where such operations are processed. This center is called the Central Complex and encompasses five different subneuropilar structures in the adult insect brain. In Schistocerca embryos and Drosophila pupae, the respective progenitor cells, so called neuroblasts, were identified. However, only little is known about the molecular genetic background that enables the formation of the Central Complex. Respective embryonic genetic studies are not possible in Drosophila, since the Central Complex develops later during larval stages. In contrast, Tribolium castaneum larvae form a reduced Central Complex during embryogenesis which is easily accessible for genetic functional studies. Therefore, Tribolium was chosen to establish a new system to investigate embryonic insect brain development.

As a prerequisite for this aim, transgenic reporter lines were established, that allow studies of brain development in Tribolium castaneum. A transgenic line, where the regulatory regions of the Tribolium homologue to reversed polarity drives the reporter gene tGFP, was generated (reg. Tcrepo::tGFP). This line 'reg. Tc-repo::tGFP' appears to mark a subset of glial cells in the larval brain. Additionally, transgenic reporter lines, which should enable tracing of neuroblast development from the onset to the respective fully developed structure in the brain were generated. For this, various putative regulatory regions of the genes $T c$-six 3 and $T c-r x$ were used to drive the reporter genes tGFP and DsRedExpress, respectively. The resulting transgenic lines where analyzed regarding embryonic expression as well as larval fluorescence patterns of the respective reporter genes. Thereby two lines were identified, which will be useful to study the development of a certain lineage. Furthermore, existing lines were characterized regarding their larval brain expression pattern. The promotor 6xP3 was shown to mark glial cells. Another line was shown to mark neural cells. Finally, a 'Mushroom Body' line derived from the GEKU screen marks the Mushroom Body neuropile. These lines were used in the second part of this work, where candidate genes were tested for a role in embryonic Central Complex formation in Tribolium. These analyses revealed that the genes $T c-r x, T c-c h x$, and $T c-s i x 3$ play important roles in different steps of the larval brain midline specification and Central Complex development in Tribolium.

Taken together, valuable tools, which allow investigations of the complex genetic network, which is needed for embryonic brain development in Tribolium castaneum were established in this work. Further, this system will allow identification of genes and studies of their function in embryonic 
Central Complex formation in Tribolium. This is especially valuable, since the Central Complex develops only at later stages in Drosophila. 


\section{Introduction}

\subsection{Conservation of the bilaterian brain}

The brain is the organ which filters, reacts to, and processes the stimuli of the environment as well as of the own body. Additionally, this organ enables complex activities like orientation, generalization, creation of strategies, and also communication.

The evolutionary origin of the central nervous system (CNS) in bilaterians has been controversially discussed. Its first appearance and its structure and function are not known. Even whether there is an independent evolutionary origin of vertebrates' and invertebrates' CNS has been debated (Arendt and Nübler-Jung, 1999; Holland, 2003; Lowe et al., 2003; Arendt et al., 2008). Recent data revealed a high degree of similarity of the molecular machinery building the brain of all bilaterian animals. This strongly indicates, that an Urbilaterian CNS precursor existed (Finkelstein and Booncinelli, 1994; Leuzinger et al., 1998; Loosli et al., 1998; Eggert et al., 1998; Acompora et al., 1999; Hirth and Reichert, 1999; Utting et al., 2000; Kammermeier and Reichert, 2001; Arendt et al., 2002; Sprecher and Reichert, 2003; Lichtneckert and Reichert, 2005; Tessmar-Raible et al., 2007; Denes et al., 2007; Arendt et al., 2008, Reichert, 2009; Hirth, 2010; Tomer et al., 2010; Steinmetz et al., 2010; Posnien et al., 2011).

The opinion that bilaterians CNS are homologous is based upon genetic patterning systems, which are conserved between vertebrates and invertebrates. There is the dorsoventral $(\mathrm{dv})$ patterning mechanism, that specifies neurogenic and nonneurogenic tissue along the dv-axis. Vertebrates exhibit a dorsally located nerve cord, while invertebrates are characterized by a ventral nerve cord. Nevertheless, genes involved in dv-axis formation are homologues, like short gastrulation (sog, Drosophila melanogaster) and Chordin (vertebrates), decapentaplegic (dpp, Drosphila melanogaster) and bone morphogenetic protein 4 (BMP4, vertebrates), and feature conserved functions. The homologue genes sog/Chordin are expressed in the early embryonic neuroectoderm by inhibiting the antineural function of the $d p p / B M P 4$ signal. Thus, the functions of the homologue groups are conserved, albeit inverted regarding the dv-axis, which is thought to be the consequence of a body axis inversion in one of the two animal groups, Deuterostomia or Protostomia (Arendt and Nübler-Jung, 1994). Even the establishment of dorsal ventral polarity within the developing CNS is conserved. The Drosophila columnar genes ventral nerve cord defective (vnd), intemediate neuroblast defective (ind), and muscle specific homeobox ( $m s h)$ are essential for the formation and specification of medial, intermedial, and lateral neuroblasts (NBs) along the anterior posterior axis (reviewed in Skeath and Thor, 2003). In vertebrates, the homologue genes $N k x 2$, Gsh, and Msx are involved in specifying the respective columns in the developing neural plate (reviewed in Arendt 
and Nübler-Jung, 1999), which could be also shown for homologues in Platynereis (Denes et al., 2007).

Not only the developing nerve cord features conserved mechanisms, but also the anterior brain development of Drosophila and mouse. Empty spiracles (ems) is a cephalic gap gene in Drosophila and is broadly expressed in blastodermal stages during embryogenesis. Loss-of-function of the ems gene results in a gap-like phenotype in the brain (Hirth et al., 1995; Hartmann et al., 2000). Finally, this gene is required for the specification and formation of the anterior part of the embryonic brain in Drosophila. However, vertebrates have two orthologues Emx1 and Emx2, which are expressed in the anterior region of the developing brain in the mouse. While a dysfunction of Emx 1 causes minor defects, a loss of Emx2 leads to immediate death after birth (Bishop et al., 2002). In Emx2 mutants, the anterior motor and sensory cortical areas are expanded caudally, whereas the posterior visual cortical areas are reduced in size (Hartmann et al., 2000; Bishop et al., 2002). Strikingly, rescue experiments with ubiquitous overexpression of Emx2 in ems mutant flies demonstrated the conserved function of these genes among the phyla (Hartmann et al., 2000).

An additional functional conservation is demonstrated by the otd/Otx genes in embryonic brain development of Drosophila and mice. In Drosophila, otd is expressed anteriorily in the brain, while the anteriormost part of the neuraxis is devoid of otd expression (Younossi-Hartenstein, 1997). Mutation analyses revealed that most of the protocerebral and deuterocerbral neuroblasts are absent, which causes the absence of the entire anterior brain (Hirth et al., 1995; Younossi-Hartenstein et al., 1997). In mice, two orthologues have been identified, Otxl and Otx2. Interestingly, these are expessed in the brain, but the most anterior part of the embryonic brain as well lacks Otx2 expression (Simeone et al., 1992). A loss-of-function of Otxl leads to minor effects (Acampora et al., 1997), while Otx2 mutant mice lack the rostral neurocetoderm (Acampora et al., 1995). Again, cross-phylum rescue experiments with the Drosophila otd cDNA sequence embedded by the $5^{\prime}$ and 3' UTR of mouse Otx2 restored the rostral brain in Otx2 null mutant mice (Acampora et al., 2001). This supports the idea that otd/Otx function is conserved and derived from a common ancestor of vertebrates and insects (Lichtneckert and Reichert, 2005).

Apparently, many mechanisms important for the brain development are conserved between mice and insects. Thus it is very likely, that additional processes and molecular mechanisms in regionalization, specification, and formation of the embryonic brain are conserved. An additional argument for insect studies is the complexity of the vertebrate brain. The highly complex structure of the vertebrate brain complicates the research of genetic mechanisms of brain development. For example, the mammalian brain consists of billions neurons (reviewed in Luo et al., 2008), $1 \mathrm{~mm}^{3}$ 
consists of 1000000 neurons, more than $4 \mathrm{~km}$ of axons, $500 \mathrm{~m}$ of dendrites, and more than 700 million synapses (Breitberg et al., 1998; Cardona et al., 2010). Compared to this, insect brains are not that large, for example Drosophila melanogaster has a small brain which develops from $~ 100$ NBs per hemisphere. Finally, 200000 neurons form the adult brain in Drosophila melanogaster (Younossi-Hartenstein et al., 1996; Urbach and Technau, 2003; Younossi-Hartenstein et al., 2006). Due to the lower complexity, it is much easier to study the general developmental processes of brain regionalization and specification in insect model organisms. Hence, in insect research it is easier to gain insight into the genetic network which is necessary for building such complex organs as the brain.

\subsection{From neuroectoderm to neuropile}

The development of the CNS in insects is a highly stereotyped process, starting with the sorting of neural and non-neural progenitors, which takes place during early embryogenesis. In Drosophila, the dorsoventral borders of the neuroectoderm get determined by the ventral to dorsal protein gradient of Dorsal (reviewed in Egger et al., 2008). On the one hand, the Dorsal-protein inhibits the expression of the gene $d p p$ ventrally and on the other hand, low levels of this protein activate the expression of the gene $\operatorname{sog}$, which outlines the presumptive neuroectoderm (reviewed in Egger et al., 2008).

The next step in CNS development is the determination of the neural stem cells which derive from neuroectodermal cells. Due to its relatively simple structure, the trunk neuroectoderm of Drosophila was chosen for studies of the processes and the underlying mechanisms regarding selection of single cells to become neuroblasts during the last decades. The neuroectoderm encompassing the ventral neurogenic region is structured by genetic patterning and segmentation cascades. I.e. the expression of pair rule, segment polarity, and hox genes determine the anterior posterior positions of NBs, while the dorsoventral axis is specified by the extracellular opponent protein gradients of Dpp and Sog, which together with EGFR signaling lead to the activation of the columnar genes (reviewed in Egger et al., 2008; Gilbert, 2003; Skeath and Thor, 2003, Stollewerk and Simpson, 2005). Eventually, the positioning of pronerual clusters and the specification of NB fate is finally based on the interaction of the pair rule, the segment polarity, and the columnar genes (reviewed in Stollewerk and Simpson, 2005).

The proneural clusters are groups of 5-7 equipotent cells, and are specified by the expression of the proneural genes acheate and scute. By this, all cells of such a cluster achieve neural competence (Skeath, 1999; Wheeler et al., 2003). However, only one cell of such a cluster is selected to become 
a NB. This selection is enabled by juxtaposed cell-cell signaling and is called the lateral inhibition (e.g. reviewed in Skeath and Thor, 2003; Urbach and Technau, 2004; Pi and Chien, 2007). This is performed by the Notch pathway, which is involved in many other developmental processes, all of which have the decision between two different fates in common (reviewed in Beatus, 1998; Portin, 2002; Wheeler et al., 2008; Axelrod, 2010). The receptor Notch and its ligands Delta and Serrat are present at the cell surface of the equipotent proneural cluster cells. Stochastic changes in the representation of the levels of ligand and receptor among these cells enable the differentiation of the epidermal and neural fate. Meaning, high levels of Notch signaling inhibit the expression of the ligand and of the proneural genes in the surrounding and therefore lateral cells, which leads to an epidermal fate. At the same time, the selected proneural cell, which expresses high levels of the ligands of Notch, forms the neuroblast (e.g. Beatus and Lendahl, 1998; Pi and Chien, 2007; Axelrod, 2010).

In the ventral neurogenic region, after selection of neuronal fate, NBs delaminate in five temporal waves. Thus, $30 \mathrm{NBs}$ derive per hemisegment, each forming a nearly invariant and unique cell lineage. The NB fate is specified by the position and the time of formation in the neuroectoderm (Urbach and Technau, 2004; Technau et al, 2005; Stollewerk and Simpson, 2006; Hartenstein et al, 2008). Shortly after delamination, these neuronal stem cells initiate asymmetrical and invariant cell division, thereby renewing themselves and creating a smaller daughter cell, the so called ganglion mother cell (GMC). The primary NBs divide 5-8 times in a stem-cell like manner, while the GMCs divide only once, 60-90 min after birth. By this final symmetrical division, the two daughter cells are differentiated postmitotically into glia and/or neuronal cells (Hartenstein et al., 1987; Hartenstein et al., 2008).

Similar to the trunk NBs, the formation of the brain NBs follows a reproducible pattern, where each NB is generated at a characteristic time (Urbach and Technau, 2004). For Drosophila melanogaster, it is known that 106 NBs are forming the brain. The protocerebrum takes the largest portion of NBs with 72, and gives rise to various neuropiles (see below). The deutocerebrum is associated with the antennal segment, where $21 \mathrm{NBs}$ arise. Finally, the tritocerebrum encompasses $13 \mathrm{NBs}$ in Drosophila, and represents the neuromere of the intercalary segment (reviewed in Urbach and Technau, 2003). While in the ventral neurogenic regions 30 NBs arise per hemisegment, 100 NBs derive from the procephalic neurogenic region and form the brain (Younossi-Hartenstein et al., 1996; Urbach and Technau, 2003; Urbach et al., 2003). The ratio of cells with ectodermal and neuronal fate differs significantly between head and trunk neuroectoderm (Schmidt-Ott and Technau, 1994; Urbach and Technau, 2004). This is due to a less stringent lateral inhibition in the 
procephalic neuroectoderm, where NBs, that derived from one pronerual cluster were identified (Urbach et al 2003; Urbach and Technau, 2003; Urbach and Technau, 2004;Technau et al., 2006). A further difference between ventral and proecephalic neurogenic region is that the brain NBs do not appear in waves, but rather continuously (Younossi-Hartenstein et al., 2006).

\subsubsection{Specification of neuroblast identity}

In Drosophila, the individual identity of the $30 \mathrm{NBs}$ per hemisegment in the ventral neurogenic region is given by the spatial and temporal pattern of their delamination. The grid-like expression pattern of genes along the anterioposterior (segment polarity) and dorsoventral (columnar genes) axes subdivides the trunk hemisegment. Thus, NBs which arise in the respective quadrant of each hemisegment are serial homologues. Additionally, such serial homologues express the same combination of molecular markers and give rise to almost identical cell lineages (reviewed in Urbach and Technau, 2003; Urbach and Technau, 2004; Sprecher et al., 2007). Taken together, segment polarity and dorsoventral patterning genes in combination with temporal signals in the neuroectoderm explain how each arising truncal NB acquires an individual fate (Doe and Goodman, 1985; Technau et al., 2006)

However, the mechanisms of determining the different identities of the brain NBs are still unknown. Analogous expression of segment polarity genes and dorsoventral patterning genes occurs in specific domains in the procephalic neuroectoderm before NBs delaminate. Therefore it is discussed, that these genes are required for providing positional information and specification of brain NBs in analogy to the specification of trunk NBs (reviewed in Urbach and Technau, 2004). However, the segmental character, i.e. the expression of segment polarity genes and dorsoventral patterning genes, is less conserved for the procephalic neuromeres. Especially the protocerebrum comprises very little homology to truncal neuromeres compared to the deuterocerebrum and tritocerebrum. Due to this, the existence of serially homologous NBs in the anterior protocerebrum is less evident (Urbach and Technau, 2004; Technau et al., 2006). Nevertheless, the formation of NBs in the procephalic neuroectoderm follows a reproducible pattern. Urbach and Technau (2003) analyzed the expression pattern of more than 40 molecular markers, including proneural genes, segment polarity genes, dorsoventral patterning genes, and others, in the procephalic neuroectoderm of Drosophila (Figure 1). This study revealed that each of the $\sim 100$ brain NBs express a unique combination of transcription factors. This complex expression pattern is strongly suggested to support the machinery leading to the specification of individual NB fate, but the genetically relevant 
network has not been identified yet (Urbach and Technau, 2003; Urbach and Technau 2004; Technau et al., 2006).

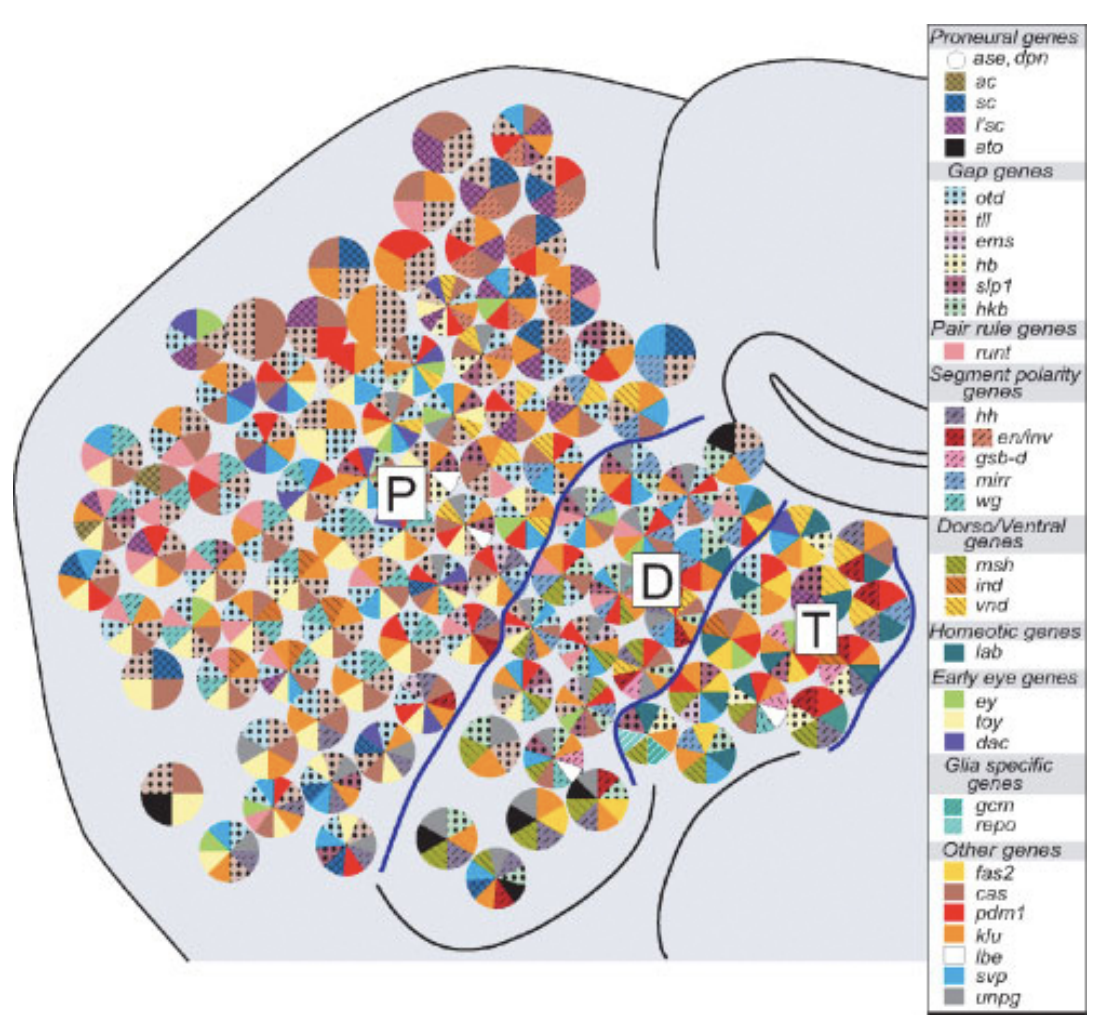

Urbach and Technau, 2003; Urbach and Technau, 2004)

Figure 1: Combinations of marker gene expression reflect individual identities of brain NBs in Drosophila. Schematic presentation of about 100 NB populations of a Drosophila embryo. NBs are depicted as equal-sized circles at positions roughly corresponding to their positions in situ. More than 40 different molecular markers have been found to be specifically expressed in subsets of brain NBs. Depicted are 34 different genes, listed in the box on the right. Each brain NB reveals an individual combinatorial code of marker gene expression, which uniquely identifies each NB. Blue lines indicate the segmental boundaries between the tritocerebrum $(\mathrm{T})$, deutocerebrum (D) and protocerebrum $(\mathrm{P})$.(modified from

\subsubsection{Neuroblast lineages}

Tracing the development from NBs in the neuroectoderm of embryos to the respective neuropile in the adult brain was possible only in few cases so far. Younossi-Hartenstein and colleagues (2006) were able to establish a map of primary axon bundles of NBs and their progenies within the brain cortex of Drosophila embryos of stage 15 and 16. The progenies of NBs remain together in the cortex of the developing brain and form small cell clusters. Upon differentiation, they start axogenesis in a collective manner, therefore the neurons of one cone form a joint primary axon bundle (Younossi-Hartenstein et al., 2006). By mapping all bundles, it was possible to reveal which primary axon bundle contributed to which neuropile and partially from which NB in the cortex such bundles derived. This study enabled an investigation of the complex development of 'macrocircuits' in the developing embryonic brain, as well as the development from the larval to the adult brain of Drosophila (Younossi-Hartenstein et al., 2006; Hartenstein et al., 2008, Fung et al., 2009; Larsen et al., 2009; Spindler et al., 2009; Kumar et al., 2009; Cardona et al., 2009; Cardona et al., 2010). The first identified NBs which were localized in the neuroectoderm and functionally analyzed, were those of the Mushroom Body (MB). In previous studies, the NBs and their progenies could be 
localized in the embryonic and larval brain, and it was shown that there were 4 NBs per hemisphere (e.g. Technau and Heisenberg, 1982; Ito and Hotta, 1992; Tettmani et al., 1997). Noveen and colleagues (2000) identified an important function of the genes dachshund and eyeless for the proper development of the Mushroom Body. Finally, they were able to trace the Mushroom Body NBs from the embryonic neuroectoderm to the adult Mushroom Body neuropile.

An additional NB lineage which could be traced from the embryo to the adult brain in Drosophila, was positive for engrailed. Kumar and colleagues (2009) could trace and analyze the engrailed positive serial homologue NBs within the procephalic neuromeres. By tracing these NBs to adulthood, they showed that engrailed-positive neurons of differing neuromeric origin do not project into neuromerically different compartments in the brain. Additionally, in embryonic, larval and adult stages, these neurons innervate the same neuropile compartments.

Thus, only few NBs could be traced from their origin within the procephalic neuroectoderm to the architectural structure within the adult brain so far. Especially the question of the genetic regulation of the protocererbral NB identity is still incompletely understood.

\subsection{The insect brain}

Already during the nineteenth century, the insect brain was studied and described based on investigations by bright field microscopy (e.g. Flögel 1876 and 1878; Weismann, 1884; Johansen, 1892; Wheeler, 1893; Kenyon, 1896). Here, the brain architecture was described morphologically. In the last decades, Drosophila melanogaster and Schistocerca gregaria were the most studied organisms regarding brain development and especially regarding the question which function can be attributed to the brain compartments (Olsen and Wilson, 2008; Hong et al., 2008; Johard et al., 2008; Pan et al., 2009; Heinze and Homberg, 2009; Young and Armstrong, 2010; Perenau et al., 2010; El Jundi et al., 2010; Zill, 2010; Ayali and Lange, 2010; Yu et al., 2010; Perenau et al., 2011; Träger and Homberg 2011).

The insect CNS derives from cells of the neuroectoderm, which consists of two major parts, the anterior procephalic neurogenic region and the ventral neurogenic region. While the ventral nerve cord derives from the ventral neurogenic region, the brain derives from the procephalic neurogenic region (reviewed in Technau et al., 2006). The procephalic neurogenic region comprises the pregnathal and gnathal segment part of the neuroectoderm. The brain is composed of two ganglions, the supraesophageal and the subesophageal ganglion, each consisting of three fused neuromeres. The subesophageal ganglion consists of the fused mandibular, maxillary and labial neuromeres. These neuromeres are structured similarly to those of the segmental ganglia of the ventral nerve 
cord. The supraesophageal ganglion consists of the three neuromeres protocererbrum, deuterocerebrum, and tritocerebrum, which derive from the preocular and ocular region in case of the protocerebrum and from the antennal and intercalary segment for deutero- and tritocerebrum, respectively (Figure 1 and 2; Snodgrass, 1935; Bullock and Horridge, 1965; Burrows, 1996; Reichert and Boyan, 1997; Dettner et al., 2003). During head developmental processes in embryonic stages, the anterior most part of the neuroectoderm shifts to a dorsal position. As a consequence the anterior protocerebrum is located, relative to the body axis, dorsally in the head (reviewed in Reichert and Boyan, 1997), i.e. the neruaxis is bent with respect to the body axis (Figure 2; reviewed in Snodgras, 1935; Reichert and Boyan, 1997; Urbach and Technau, 2003).

As described above, the supraesophageal ganglion consists of three fused neuromeres, while the protocerebrum takes the largest portion of the brain. It includes the Optical Lobes, the Mushroom Bodies, and the Central Complex and various areas in the inferior, ventral, and superior protocerebrum (reviewed in Kurylas et al., 2008).

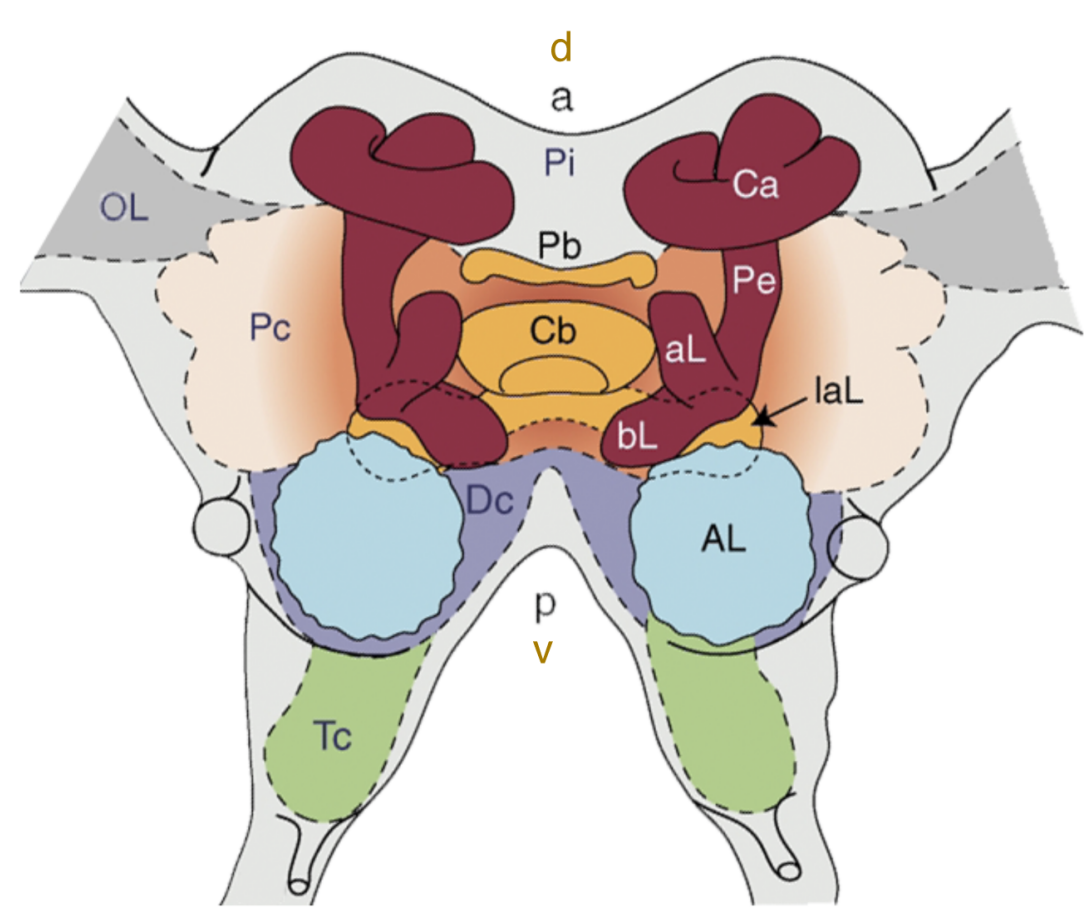

Figure 2: Scheme of an adult insect brain. The protocerebrum $(\mathrm{Pc})$ encompasses the Mushroom Bodies (dark red) with the Calyx $(\mathrm{Ca})$, Pedunculus $(\mathrm{Pe})$, with median lobes ending in the beta lobe $(\mathrm{bL})$ and the vertical lobe ending in the alpha lobus (aL). An additional unpaired neuropile in the Pc is the Central Complex (orange), which consists of the of the Protocerebral Bridge $(\mathrm{Pb})$, the Central Body $(\mathrm{Cb})$ and the lateral accessory lobes (laL). The Central Complex is mainly formed by neuroblasts located in the pars intercerebralis $(\mathrm{Pi})$. The deutocerebrum (Dc) includes the Antennal Lobe (AL; light blue). The last neuromere is the tritocerebrum (Tc). The Optical Lobes (OL; dark grey) have been indicated, but are omitted in this scheme. Relative to the neuraxis anterior (a) is top and posterior ( $\mathrm{p}$ ) is bottom; relative to the body axis (depicted in brown) dorsal (d) is top and ventral (v) is bottom. (modified from Homberg, 1994; Urbach and Technau 2003).

The two Optical Lobes are the primary visual centers of the brain. Each Optical Lobe is divided into the lamina, medulla, and lobula complex. The lobula complex is subdivided into an anterior, a dorsal, an inner, and an outer lobe (Elphick et al. 1996). Visual projection areas in the median 
protocerebrum include the anterior optic tubercles, consisting of upper and lower units. The lower unit is part of the polarization vision pathway (Homberg et al. 2003; Pfeiffer et al. 2005). The Mushroom Bodies represent a paired protocerebral brain structure which is found in all insects (Strausfeld et al., 2009). These neuropiles consist of axons of thousands of Kenyon cell fibers, which project through the peduncle $(\mathrm{Pe})$ into the distinct lobes (median and vertical lobes). The calyces $(\mathrm{Ca})$ of the Mushroom Bodies are mainly formed by dendrites of the Kenyon cells (Figure 2). The Mushroom Bodies are essential for olfactory learning and memory (reviewed in Fahrbach, 2006; Strausfeld 2009). The according NBs to this neuropile could be localized to the neuroectoderm as well as genetically characterized (see below).

The second prominent protocerebral neuropile is the Central Complex (CC; Figure 2). It is also the only neuropile complex within the insect brain, which spans the midline of the two hemispheres. The Central Complex comprises a group of neuropile modules: the Protocerebral Bridge (PB), the Central Body (CB) which consists of an upper and a lower unit, the noduli, and the lateral accessory lobes (laL) (reviewed in Homberg, 2008; Boyan and Reichert, 2011). The Central Complex is generated at different developmental stages in different arthropods. Hemimetabolous insects comprise a fully matured and developed Central Complex featuring all neuropilear modules at the end of embryogenesis (Boyan and Williams, 1997; Williams et al., 2005). Holometabolous insects, such as Tenebrio molitor possess a partially developed Central Complex at the end of embryogenesis, which successively develops postembryonically until the pupation (Wegerhoff and Breidbach, 1992). Only for the grasshopper Schistocerca and the fruit fly Drosophila, neuroblasts involved in the development of the Central Complex were identified (Boyan and Williams, 1997; Williams et al., 2005; Izergia et al., 2009). These NBs are located anterior median to the Mushroom Body, hence in the pars intercerebralis (Pi). In Schistocerca, 8 NBs, bilaterally in the brain, form clusters of progeny, which by their tracts contribute to the Central Complex (Boyan and Williams, 1997; Williams et al., 2005; Williams and Boyan, 2008; Boyan et al., 2008; Boyan and Reichert, 2011). Functionally, the Central Complex serves a role in sky compass orientation (Heinze and Homberg 2007), fine control of motoric behaviour (Martin et al., 1999; Loesel et al., 2002; Strauss, 2002; Homberg 2008, Poeck et al., 2008) and visual memory (Liu et al. 2006).

The neuromere of the antennal segment is the deutocerebrum. The Antennal Lobes are organized in globular structures, the so called glomeruli and serve as an olfactory integration center. The Antennal Lobes are connected to the calyces of the Mushroom Body and to the lateral horn of the protocerebrum. The tritocerebrum derives from the intercalary segment and is located posterior and ventral to the deutocerebrum. It connects the brain to the stomatogastric nervous system (reviewed 
in Kurylas et al., 2008)

\subsection{Tribolium castaneum as a model organism for studies of brain development}

Currently, there are mainly two insect model organisms in which the development of the neural stem cells of the brain have been studied in detail, Schistocerca gregaria and Drosophila melanogaster. In Schistocerca, embryonic brain development was analyzed by morphological studies like immunohistochemistry, dye injection, BrdU incorporation, and Wigglesworth's osmium tetroxideethyl gallate histology (cell death; e.g. Boyan et al., 1995; Boyan and Williams 1997; Boyan et al., 2002; Ludwig et al., 2002; Wiliams et al., 2005; Boyan et al., 2010). Molecular and genetical investigations are difficult, since so far Schistocerca lack a sequenced genome. However, recently the transcriptome of the central nervous system in Schistocerca gregaria was analyzed and described (Badisco et al., 2011). Nevertheless, gene function analysis is still at the beginning and was first tested by RNAi in nymphal stages (Dong and Friedrich, 2005; Dong and Friedrich, 2010). A very promising and highly developed morphological, molecular, and genetic model organism is Drosophila melanogaster. For this organism, many tools are available which allow studies of complex genetical specification and determination processes of the brain (e.g. Urbach and Technau, 2003; Edenfeld et al., 2005; Younossi-Hartenstein et al., 2006, Soustelle and Giangrande, 2006; Colonques et al., 2007; Berger et al., 2007; Spindler et al., 2009). However, the larval brain of Drosophila is reduced. For instance, this organism possess only a miniature Mushroom Body in the larval brain and lacks the Central Complex neuropile at this developmental stage (Boquet et al., 1999). Recent studies revealed that the onset of the development of the Central Complex in Drosophila melanogaster starts with the Protocerebral Bridge and the fan shaped body during the third larval instar. The Central Complex development in Drosophila melanogaster takes place during the larval to adult transition, most parts are formed and matured during puparium (Young and Armstrong, 2010). Thus, the genetic processes that specify cells forming a Central Complex are likely to be different to those during embryonic development. This means a different context and therefore, Drosophila melanogaster is unsuitable for investigating the embryonic signals required for Central Complex development.

However, a suited model organism to study this process is the red flour beetle Tribolium castaneum. Since its genome sequence is available (Richards et al., 2008) and the red flour beetle shows a robust and systemic RNAi response, functional analyses of genes by a knock down at any stage and in any tissue, is one of its largest potentials (e.g. Brown et al., 1999; Bucher et al., 2002; Tomoyasu et al., 2004; Posnien et al., 2009). The establishment of transposon mediated transgenesis in 
Tribolium castaneum offers the development of tools to gain insight into gene functions, genetic control of genes, and the complex genetic network (Berghammer et al., 1999). The first large-scale insertional mutagenesis screen in an insect outside of Drosophila was performed by a consortium of different laboratories (GEKU), where various enhancer trap lines marked with eGFP were generated (see below; Trauner et al., 2009). In addition, imaging lines have been created and gene misexpression techniques have been established (Schinko et al., 2010). Tribolium has an insect typical head during larval stages (reviewed in Bucher and Wimmer, 2005; Posnien et al., 2010). Importantly, the larval brain of Tribolium posses parts of the Central Complex neuropiles, which are lacking in the larval brain of Drosophila (see below; Young and Armstrong, 2010). Therefore, Tribolium castaneum was chosen to study the genetic mechanisms of embryonic brain development, with the focus on the Central Body development.

\subsection{The larval brain of Tribolium castaneum}

Adult Tribolium castaneum beetles have an insect typical brain, featuring all neuropilear modules, which are described above (Dreyer et al., 2010). The first instar larval (L1) brain has not been investigated so far, due to technical limitations. However, after the generation of transgenic lines with distinct fluorescence signals in the brain, analyses regarding the architecture of the larval brain became easier. This was performed in parallel to this work in the laboratory of Joachim Schachtner (University of Marburg). Here, the first larval instar brain was studied regarding the morphology of the Mushroom Body, the Central Complex, the Optical Lobes, and the Antennal Lobes. Additionally, two transgenic lines were analyzed regarding the expression pattern and tissue specificity by colocalisation analyses. These lines were the GEKU screen derived 'Mushroom Body' line (Trauner et al., 2009; chapter 3.4) and the 6xP3::eCFP line (Hein, 2007; chapter 3.4).

In contrast to the adult brain, the composition of neuropiles in the larval brain differs among insect species. Hemimetabolous insects like Schistocerca have an insect typical brain already within the first larval instar, with all neuropiles described above (Bentley and Toroian-Raymond, 1981; Homberg, 2008). Holometabolous insect larvae often show reduced neuropilear structures, like in Drosophila where the Central Complex is absent in L1 brain, but gets formed during the last larval instar (Renn et al., 1999; Young and Armstrong, 2010). In others, neuropiles are reduced, like in Tenebrio where only the upper part of the Central Complex is present (Wegerhoff and Breidbach, 1992). The large differences in the presence of the Central Complex during larval or nymphal stages between holometabolous and hemimetabolous insects were discussed to be due to diverse efforts of locomotion in legless larvae (Drosophila) and larvae with legs (Schistocerca, Tenebrio). Kollmann 
and colleagues showed, that the first larval instar brain of Tribolium castaneum mirrors the reduced situation. Furthermore, the larval brain is much more bent as the adult brain. The neuraxis in the larva is almost inversely parallel to the body axis, meaning the anterior part of the brain is localized posterior in the body, and vice versa the posterior part of the brain is localized anterior. Therefore, the descriptions of directions within the brain are given according to the directions of the body axis (Figure 3).

In the Tribolium larval brain, the Mushroom Body is already present with its typical projections of the Pedunculus $(\mathrm{Pe})$ and its branching lobes (median, $\mathrm{mL}$, and vertical, vL; Figure 3), while the Central Complex is only partially formed. As described above, the Central Complex is comprised of five modular neuropiles, the Protocerebral Bridge, the Central Body consisting of upper and lower unit, the noduli, and the lateral accessory lobes (reviewed in Boyan and Reichert, 2011). In the larval brain, the noduli and the lateral accessory lobes were not detected. The Protocerebral Bridge is not fused medially and is wedge shaped, whereas also a glial envelope was not detected (Figure 3). The Central Body is reduced, similar to that in Tenebrio larval satges. In Tribolium, it consists of one flattened structure, which by respective antibody staining was suggested to be the reduced upper unit (Figure 3; c.f. chapter 4.3.4). The Optical Lobes in larval stages are reduced, therefore they are called the anlagen of Optical Lobes (A-OL). The larval Antennal Lobes are organized in globular structures, like in the adult brain (Figure 3).

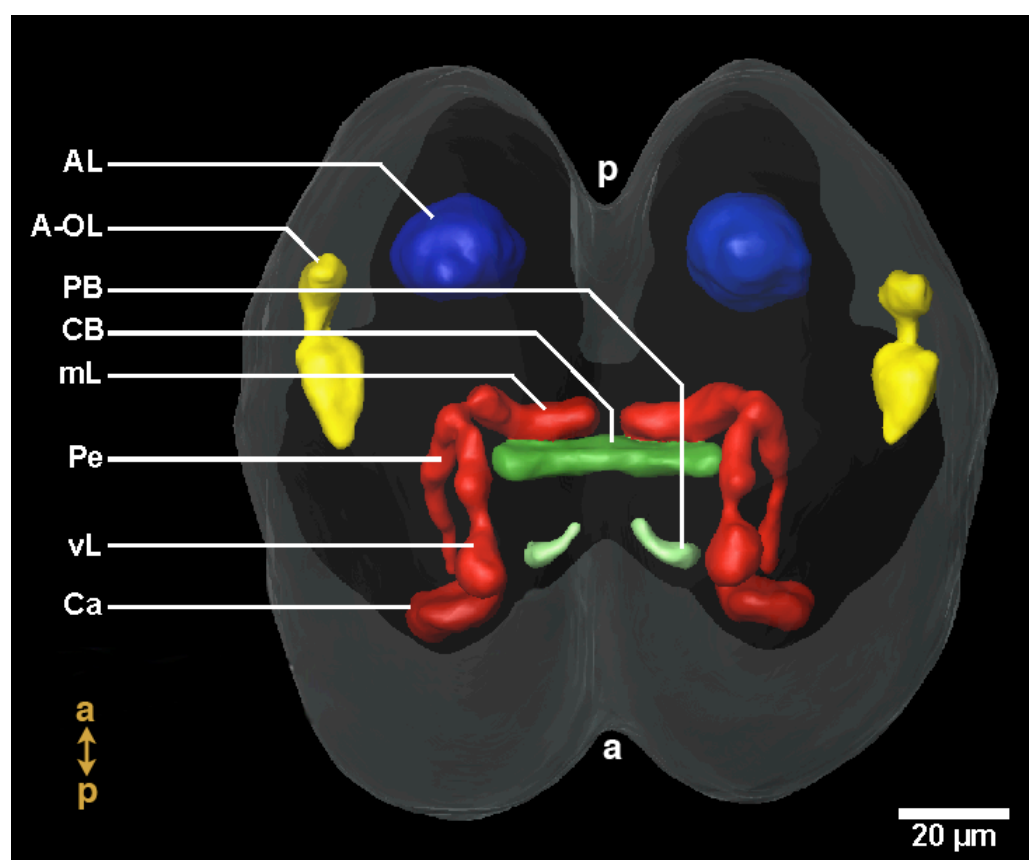

Figure 3: The larval brain of Tribolium castaneum (Martin Kollmann, University of Marburg).

The neuraxis (anterior: a; posterior: p; white letters) is inverted according to the body axis (brown letters; a and $\mathrm{p}$ ). The larval brain comprises the Antennal Lobes (AL), the anlagen of the Optical Lobes (AOL), the upper unit of the Central Body (CB), the paired Protocerebral Bridge (PB) and the Mushroom Bodies, with the calyx (Ca), the Pedunculus (Pe), and the branching median and vertical lobes $(\mathrm{mL}$; $\mathrm{vL).}$ 
The G 11410 enhancer trap line, which is derived from the GEKU screen, showed a prominent reporter gene expression in the larval Mushroom Bodies. The specificity of the tGFP expression pattern in the Mushroom Bodies was confirmed by a colocalisation assay with the antibody DcO (Farris and Strausfeld, 2003), therefore this line is further called 'Mushroom Body' line (chapter 3.4). To confirm the assumption, that the cells positive for the $6 \times \mathrm{P} 3:: \mathrm{eCFP}$ signal are glial cells, immunohistochemistry stainings were performed with an antibody against the glial marker reversed polarity (anti Repo; Campbell et al., 1994; Halter et al., 1995; Xiong et al., 1994; Lee \& Jones 2005). These analyses revealed that $98,8 \%$ of the cells positive for the eCFP signal are colocalized with the glial marker Repo, which suggests that the 6xP3::eCFP signal marks glial cells (Kollmann and Schachtner, unpublished).

\subsection{Candidate genes for NB identity specification in the insect brain}

The genes which were selected for analysis of their function in the brain development of Tribolium castaneum, are known to be expressed in the embryonic head anlage or even to play an important role in head development. The expression pattern and function of Tc-six3 and the homologues $T c$ otd1 and Tc-otd2 were known from previous studies (Schröder, 2003; Posnien, 2006; Schinko et al., 2008; Posnien, 2009) and all these genes of interest are conserved and are required for proper brain development in other species.

$T c-\operatorname{six} 3$ is a transcription factor, which encodes a homeobox and a Six domain and is the homologue to SIX3 in vertebrates or to Optix in Drosophila. This gene is conserved in bilateria and marks the anterior-most region of the developing brain (Oliver et al., 1995; Steinmetz et al., 2010). It is known from vertebrates including humans, that a loss of function of this gene leads to severe phenotypes, the so called holoprosencephaly (HPE). Here, the median interhemispheric division and structures are affected (Pasquier et al., 2000; Dubourg et al., 2007; Domené et al., 2008; Salomon et al., 2009). In Tribolium castaneum, comparable phenotypes lacking the median head region and the labrum were generated by knock down of Tc-six3 by parental RNAi (Posnien, 2006). An overexpression of the six 3 homologue in zebrafish lead to an enlargement of the rostral brain (Kobayashi et al., 1998). Analyses in Xenopus and zebrafish revealed that Six3 itself is unable to induce neural tissue and overexpression promotes cell proliferation (Gestri et al, 2005). In Drosophila, an overexpression of optix leads to ectopic eye formation (Seimiya ad Gehring, 2000). Thus, six3 homologues are involved in neural plate and brain specification, development of neuroendocrine systems, and eye development (e.g. Loosli et al., 1999; Seimiya and Gehring, 2000; Posnien, 2006; Steinmetz et al., 2010). 
The gene orthodenticle belongs to the family of paired-like homeodomain transcription factors. Homologues were identified in various species like Drosphila melanogaster, Ceratitis capitata, Anastrepha suspensa, Nasonia vitripennis, Parhyale hawaiensis, Euscorpius flavicaudis, Tegenaria saeva, Xenopus laevis, Mus musculus, and others (Finkelstein et al., 1990; Simeone et al., 1993; Andreazzoli et al., 1997; Schröder 2003; Lynch et al., 2006; Browne et al., 2006; Schetelig et al., 2008). The otd homologues play a crucial role in head segmentation, brain regionalization and also photoreceptor development (Finkelstein et al., 1990; Simeone et al., 1993; Frantz et al., 1994; Hirth et al., 1995; Andreazzoli et al., 1997; Leuzinger et al, 1998; Blanco et al., 2009). In Drosophila melanogaster, otd belongs to the head gap genes and loss of function analyses revealed a loss of protocerebrum and anterior parts of the deuterocerebrum in brain development (Leuzinger et al., 1998). An additional putative function of Drosophila otd was the specification of progenies of mesectodermal neuroblasts in the ventral nerve cord (Finkelstein et al., 1990). In Tribolium castaneum, the two paralogue genes Tc-otd1 and Tc-otd 2 were identified. Tc-otd 1 is required for the anterior embryonic patterning and it was suggested to substitute the anterior patterning system together with $T c-h b$ in short germ insects such as Tribolium, in parallel to the bicoid morphogene function in Drosophila (Schröder 2003; Schinko et al., 2008; Posnien, 2009). In additional studies, Tc-otdl function was revealed to be involved in dorsoventral axis formation in early embryonic stages of Tribolium castaneum (Kotkamp et al., 2010). A function in brain development in Tribolium castaneum was suggested, but could not be proven so far (Posnien, 2009).

Also $T c-c h x$ is a transcription factor with a homeodomain and a homologue gene to $C H X / V S X$, which is conserved among the phyla. Furthermore in Drosophila, it is known to mark the pars intercerebralis (PI), the region which is known to give rise to neuroendocrine system and the Central Complex in the insect brain (Boyan and Williams, 1997; de Velassco et al., 2007). In Tribolium, it was suggested to be a target gene of Tc-six3 (Posnien et al., unpublished). Dysfunction of $C H X 10$ in vertebrates is known to cause malformations in the visual system, in Drosophila a similar function was identified for the homologous $V_{S x} 1$ and $V_{s} x 2$ genes (Percin et al., 2000; Erclik et al., 2008; Gonzalez-Rodriguez et al., 2010). In Tribolium castaneum, one Tc-chx homologue was identified (Posnien, 2009) and a function in brain development has not been described so far.

The transcription factor retinal homeobox ( $R x /$ rax $)$ was originally discovered in vertebrates, where it plays a role in eye and brain development. The homeobox $r x$ or $\operatorname{Rax}$ is conserved throughout various species like Drosophila, Platynereis, zebrafish, chicken, Xenopus, mouse, and humans (Eggert et al., 1998; Chuang et al., 1999; Ohuchi et al., 1999; Tucker et al., 2001; Tessmar-Raible et al., 2007). It encodes for a transcription factor featuring an N-terminal octapeptide domain, a 
homeodomain, a $R x$ domain, and a C-terminal orthopedia-aristaless- $r x$ domain (Eggert et al., 1998; Mathers et al., 1997, Davis et al., 2003). It is known to play major roles in eye development in vertebrates (Mathers et al., 1997; Voronina et al., 2004; Loosli et al., 2003; Rojas-Munoz et al., 2005). Homologues of $r x$ were also discussed to be involved in forming the neuroendocrine system in Drosophila, Platynereis, Zebrafish, and other animals (Hartenstein, 2006; de Velasco et al., 2007; Tessmar-Raible et al, 2007).

\subsection{Aims of the study}

Before this work, functional studies of the larval brain were not feasible in Tribolium castaneum and therefore two aspects are encompassed: 1) Establishment of in vivo imaging systems for the analysis of embryonic brain development, in order to allow visualization of brain phenotypes and 2) functional analyses of several candidate genes with respect to brain development.

As a prerequisite for the analysis of NB lineages, transgenic lines marking a subset of NBs and their progenies should be established and characterized. Due to the exclusive anterior expression and their conservation in vertebrates, the NBs expressing the genes $T c-r x$ and $T c$-six 3 were chosen. Various reporter lines, carrying upstream and downstream regions of the respective genes, were to be created. These lines should enable tracing of the development from the NB to the respective structure in the larval brain.

For analyzing the brain of animals in WT and knock down conditions, imaging lines, which mark neural, glial, and other specific structures of the brain were to be created and used. To this end, imaging lines driving various reporter genes under the control of the regulatory region of $T c$ embryonic lethal abnormal vision (elav) and Tc-reversed polarity (repo) were to be created. Furthermore the existing lines 'Mushroom Body', derived from the GEKU screen, '6xP3::eCFP', created from H. Hein (2007), and 'EFII::DsRed', created by M. Averof, were supposed to be characterized regarding their expression pattern in the larval brain. These lines allowed functional studies with genes, which by their anterior median expression were likely to contribute to central complex development. Those genes were Tc-rx, Tc-otd1, Tc-six3, and Tc-chx.

Taken together, this work aimed at providing a basis for the investigation of the complex genetic network of embryonic brain and Central Complex development in Tribolium castaneum. 


\section{Material and Methods}

\subsection{Tribolium castaneum strains used as source of genomic DNA and for embryonic transgenesis}

For the amplification of regulatory regions, the isolated genomic DNA of animals of the San Bernardino (SB) strain (Richards et al., 2008) was used. For transgenesis, the embryos of the whiteeyed Tc-vermilion line were used (Berghammer et al., 2009).

\subsection{Amplification of the 5'UTR by RACE-PCR}

For RACE experiment, the SMART ${ }^{\mathrm{TM}}$ RACE cDNA Amplification Kit (Clontech Laboratories, Inc.) was used in combination with the Advantage 2 PCR Kit (Clontech Laboratories, Inc.). When generating first strand 5'-RACE Ready cDNA, the instructions of the SMART ${ }^{\mathrm{TM}}$ RACE cDNA Amplification Kit (Clontech Laboratories, Inc.) were followed. The respective mRNA was isolated following the instructions of the mRNA Isolation Kit (Roche). For the amplification of the 5'UTRs the instructions of the SMART ${ }^{\mathrm{TM}}$ RACE cDNA Amplification Kit (Clontech Laboratories, Inc.) were followed. The manufacturer's instructions for the kits were followed regarding PCRprograms. In case of the Tc-elav 5'UTR, a semi nested PCR was performed, where $1 \mu 1$ of the PCR products of the first RACE-experiment were used as template for the consecutive. The PCRprogram was for the gene specific primer (Table 1) under $70^{\circ} \mathrm{C}$, additionally the gene specific primers for the second RACE-PCR were of the same primer batch. For Tc-rx and Tc-six 3 external and nested primers were designed. One set of primers allowed successful amplification of the 5'UTR of Tc-rx in two independent approaches (Table 1). With two sets of primers it was possible to amplify the 5'UTR of Tc-six3 in independent approaches. Independent approaches here means the use of two different RACE 5'-cDNA pools.

Table 1: Primers used for 5'UTR amplification

\begin{tabular}{|c|c|c|}
\hline gene/primername & RACE primer (5'-3') & temperature \\
\hline$T c$-elav/RACE5_elav & CCATTCTGTTGTCCAACAACCCCCGAGT & $69,0^{\circ} \mathrm{C}$ \\
\hline$T c$-rx/rx_RACE3 & CGAGTCCGAGTATGTTGTCGATGGTGTGCCTG & $72,3{ }^{\circ} \mathrm{C}$ \\
\hline$T c$-rx/rx_RACE5 & CGATGGTGTGCCTGGGTCCGGTTCC & $72,7^{\circ} \mathrm{C}$ \\
\hline$T c$-six3/Six3_RACE5b & GAAGTTGCCGCTGTGGAAGCAGA & $66,5^{\circ} \mathrm{C}$ \\
\hline$T c$-six3/Six3_RACE4 & GCCACCGGCAGCGACCAGA & $68,8^{\circ} \mathrm{C}$ \\
\hline$T c$-six/Six3_RACE7 & GCGGAAGTTGCCGCTGTGGAAGCAGACGATG & $73,9{ }^{\circ} \mathrm{C}$ \\
\hline$T c$-six3/Six3_RACE6 & GGTGGGCCACCGGCAGCGACCAGA & $74,8^{\circ} \mathrm{C}$ \\
\hline
\end{tabular}




\subsection{Amplification and cloning of regulatory regions}

Regulatory regions of the genes $T c$-repo, Tc-elav, $T c-r x$, and $T c$-six 3 were amplified from gDNA isolated from animals of the San Bernardino strain. Isolation of gDNA was performed after Trauner and colleagues (2009). The primers included recognition sites for restriction enzymes cutting externally of their recognition sequence, thereby generating new compatible ends for cloning (small letters, Table 2). Amplified sequences were cloned into the Dual Promoter $\mathrm{pCR}^{\circledR} \mathrm{II}$ vector by using the TA Cloning ${ }^{\circledR}$ Kit (Invitrogen). The final constructs were designed with the following sections from $5^{\prime}$ to $3^{\prime}:$ 1) regulatory regions, 2) endogenous promoter, 3) reporter gene (Table 2). Care was taken to keep the linker sequence free of additional translation start sites (ATG). In case of the regulatory regions of Tc-repo, Tc-elav, 'Tc-rx 5up', and 'Tc-six3 5up', the amplification was performed including the endogenous promoter sequence. The respective reporter genes were amplified from other constructs by using according primers (Table 3). The constructs were designed and created in the vector pslfa1180fa. Afterwards, the cassette including the regulatory region, promoter, reporter gene, and SV40 was transferred into the piggyBac[3xP3; Tc-ver; SV40]fa transformation vector by using the restriction enzymes AscI and/or FseI (New England BioLabs, Inc.). The 5'-ends of the regulatory regions and the promotor-reporter gene-region of achieved constructs were sequenced at Macrogen (Korea) using the primers listed in Table 4.

Table 2: Primers used for the amplification of regulatory regions including restriction sites

\begin{tabular}{|c|c|c|c|c|c|}
\hline $\begin{array}{l}\text { regulatory } \\
\text { region }\end{array}$ & forward primer $\left(5^{\prime}-3^{\prime}\right)$ & backward primer $\left(5^{\prime}-3^{\prime}\right)$ & $\begin{array}{c}\text { restriction } \\
\text { enzyme }\end{array}$ & $\begin{array}{l}\text { fragment } \\
\text { size }[b p]\end{array}$ & $\begin{array}{c}\text { reporter } \\
\text { gene }\end{array}$ \\
\hline Tc-repo & $\begin{array}{l}\text { GTCAGTCCTTTGTGAAATC } \\
\text { ACGG }\end{array}$ & $\begin{array}{l}\text { cgagctcGATTGGCAATTGCTT } \\
\text { AATGAACC }\end{array}$ & Sac I & 4287 & tGFP \\
\hline Tc-elav & $\begin{array}{l}\text { CGCTGAAAAATTAATAAGG } \\
\text { ACCC }\end{array}$ & $\begin{array}{l}\text { TCTAGAACTTCCTCAATGG } \\
\text { TGGC }\end{array}$ & & 2551 & mRFP \\
\hline$T c-r x$ Prom & $\begin{array}{l}\text { acggatccTCCTCTAATTATAGC } \\
\text { TGAATACAGAGCCGA }\end{array}$ & $\begin{array}{l}\text { atctacgctagcCTTCACAACGGT } \\
\text { CCGATTCTATCGC }\end{array}$ & BamHI, NheI & 625 & \\
\hline$T c-r x$ 10up & $\begin{array}{l}\text { acctgaggACAGCAGCGACTC } \\
\text { GTCACTCTGA }\end{array}$ & $\begin{array}{l}\text { acggatccATTTGATAACAACG } \\
\text { GCAACATACGTCGACA }\end{array}$ & Eco81I, BamHI & 4757 & DsRedEx \\
\hline$T c-r x$ up & $\begin{array}{l}\text { acggatccGGGATCAAGCGTAA } \\
\text { ATGGGACGTCCCATACAATA }\end{array}$ & $\begin{array}{l}\text { atctacgctagcCTTCACAACGGT } \\
\text { CCGATTCTATCGC }\end{array}$ & BamHI, NheI & 6090 & DsRedEx \\
\hline $\begin{array}{l}T c-r x \\
\text { Intron }\end{array}$ & $\begin{array}{l}\text { acctgaggCACCGTAATTACGC } \\
\text { AAAGATAACAGACTCCA }\end{array}$ & $\begin{array}{l}\text { acggatccCAGGGCGGTAATCA } \\
\text { AGCCGACA }\end{array}$ & Eco81I, BamHI & 7398 & DsRedEx \\
\hline $\begin{array}{l}\text { Tc-six } 3 \\
\text { Prom }\end{array}$ & $\begin{array}{l}\text { cacagatctGTGTACACCGGTCC } \\
\text { TAAAGGTTTGAAATGA }\end{array}$ & $\begin{array}{l}\text { cacggtaccATGGGTTGCCAGC } \\
\text { TTGGGCTAGTGCACCATC }\end{array}$ & BglII,KpnI & 657 & \\
\hline $\begin{array}{l}\text { Tc-six3 } \\
\text { 10up }\end{array}$ & $\begin{array}{l}\text { accgtctcggatccTCCATGCCCAT } \\
\text { GGTGAATGGAGCACA }\end{array}$ & $\begin{array}{l}\text { accgtctcagatctCTGACAAGTG } \\
\text { CCGACAACGCTATTATCATA }\end{array}$ & $\begin{array}{l}\text { Esp3I/BamHI, } \\
\text { Esp3I/BglII }\end{array}$ & 5416 & tGFP \\
\hline
\end{tabular}


2. Material and Methods

\begin{tabular}{|c|c|c|c|c|c|}
\hline $\begin{array}{l}\text { regulatory } \\
\text { region }\end{array}$ & forward primer $\left(5^{\prime}-3^{\prime}\right)$ & backward primer $\left(5^{\prime}-3^{\prime}\right)$ & $\begin{array}{c}\text { restriction } \\
\text { enzyme }\end{array}$ & $\begin{array}{l}\text { fragment } \\
\text { size [bp] }\end{array}$ & $\begin{array}{c}\text { reporter } \\
\text { gene }\end{array}$ \\
\hline$T c-\operatorname{six} 35$ up & $\begin{array}{l}\text { cacagatctTTGGGCACCCGGA } \\
\text { ACATTTCTCGAGCAATA }\end{array}$ & $\begin{array}{l}\text { cacggtaccATGGGTTGCCAGC } \\
\text { TTGGGCTAGTGCACCATC }\end{array}$ & BglII,KpnI & 4133 & tGFP \\
\hline $\begin{array}{l}T c-s i x 3 \\
5 \text { down }\end{array}$ & $\begin{array}{l}\text { acgaagacaggatccGATGCGAGA } \\
\text { TGGAGCAGATTAATCACTA }\end{array}$ & $\begin{array}{l}\text { acgaagacgagatctCGCCACTGA } \\
\text { CTTGCTCTCTGAG }\end{array}$ & $\begin{array}{c}\text { BpiI/BamHI, } \\
\text { BpiI/BglII }\end{array}$ & 4373 & tGFP \\
\hline $\begin{array}{l}T c-\text { six } 3 \\
\text { 10down }\end{array}$ & $\begin{array}{l}\text { accgtctcggatccAAGGATTCGA } \\
\text { CAAATTCGCTGTCAGAC }\end{array}$ & $\begin{array}{l}\text { accgtctcagatctTTCTGAAGATT } \\
\text { CACGATGACTTGCTCTGTA }\end{array}$ & $\begin{array}{c}\text { BpiI/BamHI, } \\
\text { BpiI/BglII }\end{array}$ & 5246 & tGFP \\
\hline
\end{tabular}

Table 3: Primers for the amplification of reporter genes

\begin{tabular}{|c|c|c|c|c|}
\hline reportegene & froward primer (5'-3') & backward primer (5'-3') & size & annealing temperature \\
\hline tGFP & ATGGAGAGCGACGAGAGC & ATTCTTCACCGGCATCTGCATC & $697 \mathrm{bp}$ & $62^{\circ} \mathrm{C}$ \\
\hline DsRedExpress & ATGGCCTCCTCCGAGGA & CTACAGGAACAGGTGGTGG & $678 \mathrm{bp}$ & $62^{\circ} \mathrm{C}$ \\
\hline mRFP & ATGGCCTCCTCCGAGGAC & ATTAGGCGCCGGTGGAGTG & $679 \mathrm{bp}$ & $64^{\circ} \mathrm{C}$ \\
\hline
\end{tabular}

Table 4: Sequencing primers of the pre-reporter gene regions

\begin{tabular}{|c|c|}
\hline constructs & pre-reporter gene region $\left.\mathbf{( 5}^{\prime} \mathbf{3}^{\prime} \mathbf{}\right)$ \\
\hline for $T c$-six 3 reporter lines & GGTGCTCTTCATCTTGTTGGTCA \\
\hline for $T c-r x$ reporter lines & CTTGAAGCGCATGAACTCCTTGA \\
\hline for $T c$-six 3 overexpression & CGTACGGTATATATCACGTCAGTCT \\
\hline
\end{tabular}

\subsection{Whole mount in situ hybridization}

WT embryos (SB-strain) were used for Neuroblast (NB) quantification and for assembling the endogenous expression pattern of the genes Tc-chx and Tc-repo. For NB quantification, staged egg lays were used of slots of 12-18h, 18-24h, and 24-30h old embryos. Native expression pattern analyses were performed with staged egg lays of 0-24h, 24-48h, and 48-72h.

The generated reporter lines were analyzed by in situ hybridization with an antisense probe against the respective reporter gene, and staged egglays were used $(0-24 \mathrm{~h}, 24-48 \mathrm{~h}$, and $48-72 \mathrm{~h})$. In all cases, embryo rearing was performed at $32^{\circ} \mathrm{C}$. Single (NBT/BCIP) and double in situ stainings (NBT/BCIP and INT/BCIP) were performed as described previously (Schinko et al., 2009). For the used probes of genes see Table 5 . 
Table 5: Antisense probes of genes used for in situ hybridization

\begin{tabular}{|c|c|c|}
\hline Gene & Length [bp] & Labeling \\
\hline$T c$-ase & 737 & Dig/Flu \\
\hline$T c-c h x$ & 787 & Dig/Flu \\
\hline DsRedEx & 678 & Dig \\
\hline$T c-r e p o$ & 920 & Dig \\
\hline$T c-r x$ & 827 & Dig/Flu \\
\hline$T c-s i x 3$ & 1394 & Dig/Flu \\
\hline tGFP & 697 & Dig \\
\hline$T c-w g$ & 1100 & Flu \\
\hline
\end{tabular}

\subsection{Embryonic and pupal knock down of gene function by RNAi}

Templates were prepared by PCR with T7-primers from plasmid templates of full length Tc-six3 (1394 bp), Tc-otdl (1116 bp), Tc-chx (787 bp), and Tc-rx (827 bp) (Posnien et al., 2009). DsRNA was produced using the Megascript T7 Kit (Ambion). Final dsRNA concentrations between 2-5 $\mu \mathrm{g} / \mu \mathrm{l}$ were diluted to the desired concentration between $100 \mathrm{ng} / \mu \mathrm{l}$ and $3,8 \mu \mathrm{g} / \mu \mathrm{l}$, whereas an aberrance of $10 \%$ was accepted (Table 6).

For embryonic RNAi, the protocol of Berghammer et al. (2009) was used as orientation (see variations in 2.6 embryonic injections). Embryos were collected for $1-2 \mathrm{~h}$, the respective beetle strains were kept on $32^{\circ} \mathrm{C}$. Breeding for the various ages was performed at $32^{\circ} \mathrm{C}$ and embryos were finally injected with Tc-six3 and Tc-otd1 dsRNA (Table 6; Tc-otd1: 3.2.2; Tc-six3: 3.2.3). Pupal RNAi was performed according to Posnien et al. (2009) and for this, various dsRNA and concentrations were used to inject female pupae (see Table 6).

For analyzing RNAi efficiency, cuticles were scanned for malformations and defects. Cuticle clearing of the respective embryos was performed in a $1: 1$ Hoyer's medium (lactic acid) at $65^{\circ} \mathrm{C}$ over night.

Table 6: Embryonic (emb.) and pupal (pup.) RNAi of different genes

\begin{tabular}{|c|c|c|c|}
\hline dsRNA of gene & concentration & emb. RNAi & pup. RNAi \\
\hline$T c$-six3 & $100 \mathrm{ng} / \mu \mathrm{l}$ & & $\mathrm{X}$ \\
\hline & $1 \mu \mathrm{g} / \mu \mathrm{l}$ & $\mathrm{X}$ & $\mathrm{X}$ \\
\hline & $2 \mu \mathrm{g} / \mu \mathrm{l}$ & & $\mathrm{X}$ \\
\hline$T c$-otd1 & $1 \mu \mathrm{g} / \mu \mathrm{l}$ & $\mathrm{X}$ & \\
\hline
\end{tabular}




\begin{tabular}{|c|c|c|c|}
\hline dsRNA of gene & concentration & emb. RNAi & pup. RNAi \\
\hline$T c-c h x$ & $1,9 \mu \mathrm{g} / \mu \mathrm{l}$ & & $\mathrm{X}$ \\
\hline & $3,8 \mu \mathrm{g} / \mu \mathrm{l}$ & & $\mathrm{X}$ \\
\hline$T c-r x$ & $1 \mu \mathrm{g} / \mu \mathrm{l}$ & & $\mathrm{X}$ \\
\hline
\end{tabular}

\subsection{Embryonic transgenesis}

For transgenesis, embryos of the white-eyed Tc-vermilion-white strain were used. The embryos were collected at $25^{\circ} \mathrm{C}$ for $1 \mathrm{~h}$ and further reared for $1 \mathrm{~h}$ at $25^{\circ} \mathrm{C}$. At the time of injections, embryos were aged $1-2 \mathrm{~h}$ at $25^{\circ} \mathrm{C}$. For injections, embryos were bleached for $1,5 \mathrm{~min}$ in $1 \%$ Danklorix (Colgate Palmolive $\mathrm{GmbH}$ ). Constructs used for injections included the positive selection marker 3xP3::Tc-vermillion, rescuing black eyes in $T c-v w$ background, or 3xP3::eGFP, causing green fluorescent eyes (Table 7). To maintain a humid atmosphere, injected embryos were reared on apple agar plates at $32^{\circ} \mathrm{C}$. Further steps and treatments were performed as described (Berghammer et al., 2009). The heat shock dependent Tc-six3 over expression construct (Table 7) was designed and cloned by Schanda (2007), but was injected and analyzed in this work.

Table 7: Injected constructs and their positive selection marker

\begin{tabular}{|c|c|}
\hline injected constructs & positive selection marker \\
\hline pBac[3xp3,g-Tc`v,SV40; Tc'repo,tGFP,SV40] & 3xP3::Tc-vermilion \\
\hline pBac[3xp3,g-Tc`v,SV40; Tc'elav,mRFP,SV40] & 3xP3::Tc-vermilion \\
\hline pBac[3xp3,g-Tc'v,SV40; Tc'rx-5up,DsRed-Ex,SV40| & 3xP3::Tc-vermilion \\
\hline pBac[3xp3,g-Tc`v,SV40; Tc'rx-10up, rx-Prom; DsRed-Ex,SV40| & 3xP3::Tc-vermilion \\
\hline pBac[3xp3,g-Tc`v,SV40; Tc'rx-Intron, rx-Prom; DsRed-Ex,SV40| & 3xP3::Tc-vermilion \\
\hline pBac[3xp3,g-Tc`v,SV40; Tc'six3 5 up, tGFP, SV40] & 3xP3::Tc-vermilion \\
\hline pBac[3xp3,g-Tc`v,SV40; Tc'six3 10 up,Prom,tGFP,SV40 ] & 3xP3::Tc-vermilion \\
\hline pBac[3xp3,g-Tc'v,SV40; Tc'six3 5 do,Prom,tGFP,SV40 ] & 3xP3::Tc-vermilion \\
\hline pBac[3xp3,g-Tc'v,SV40; Tc'six3 10 do,Prom,tGFP,SV40 ] & 3xP3::Tc-vermilion \\
\hline pBac[3xp3, eGFP,SV40; hsp5'UTR, DsRedEx, 3'UTR; hsp5'UTR, Te'six3 ORF, 3'UTR] & 3xP3::eGFP \\
\hline
\end{tabular}




\subsection{Larval brain in vivo imaging}

For scanning larval brains of transgenic lines, offspring was collected for $1-2 \mathrm{~d}$ at $32^{\circ} \mathrm{C}$. Breeding was allowed for $3,5-4 \mathrm{~d}$ at $32^{\circ} \mathrm{C}$. Embryos were bleached for 2,5 min in 1\% Danklorix (Colgate Palmolive $\mathrm{GmbH}$ ) and cleaned with water. To avoid dehydration during preparation, the mesh with embryos was kept on apple agar plates. In case of embryonic RNAi, animals were treated as described above (chapter 2.5).

Preparation of the larvae differed depending on the previous treatment. In case of transgenic lines, larvae hatched independently and only freshly hatched larvae were collected and prepared. In general, strongly affected RNAi treated animals did not hatch. By using the Leica MZ16FA fluorescence stereomicroscope, animals being close to or being hatching could be identified by two criteria: 1) a deformed egg shape, which was caused by the effort of hatching, and 2) embryos reacted with strong movements when touched. Animals not showing these characteristics had not concluded processes like dorsal closure or cuticle secretion. Embedding such animals in 80\% glycerol (Sigma-Aldrich) immediately lead to dehydration and denaturation of tissues. For those close to hatching, the vitelline membrane was removed by using fine needles and eyelash. To facilitate preparation, embryos were transferred into a drop of $80 \%$ glycerol.

To avoid crushing the larval brain while scanning, two file reinforcement rings glued on top of each other were used as spacer between slide and coverslip. They were cut into quarters and fixed on the object slide (Dippel personal communication; Dreyer et al., 2010). The distance between the pieces was chosen according to the size of the coverslip (12x12 mm). The larva was arranged dorsal up in the center of an object slide prepared this way. After covering, the larva was fixed in this position by slight pressure on the cover slide during embedding with $80 \%$ glycerol from the side.

An alternative preparation was used for more fragile animals. Those were prepared within the vitelline membrane and standing on the abdomen. Forcing them into position, they were embedded by surrounding them with small pieces of apple agar, keeping only the head free in order to avoid signal disturbance during the scan. The corners of the coverslip were prepared with plasticine to avoid crushing the embryo or larva, respectively. Drops of $80 \%$ glycerol were given right on top of the head before covering.

In vivo brain scans were documented with a Zeiss LSM 510 and processed using the Zeiss LSM Image Browser (Version 4.2.0.121). Z-projections of substacks sized 10-40 slides were prepared using ImageJ (version $1.40 \mathrm{~g}$ ) with the algorithm "average intensity“. 


\subsection{Immunohistochemistry on larval brains}

Larval brains of the transgenic line 'Brainy' (see below, 3.1.4) were dissected using a Zeiss SteREO Lumar V12. When selecting embryos for brain dissections, the same criteria as in 2.7 were chosen. Brains of embryos almost hatching were considered to be fully developed like L1 brains.

'Brainy'-WT and -RNAi larvae were transferred into a drop of cold PBS on a cover slide. Here, the abdomen was cut between the thoracic segment T3 and the abdominal segment A1. Afterwards, the brain was pressed out of the head capsule by fixing the anteriormost tissue with one pair of forceps and slightly pushing the brain to the abdominal opening with another pair of forceps. Dissected brains were collected in cold 4\% formaldehyde (Roth, Karlsruhe)/PBS up to $60 \mathrm{~min}$, followed by fixation for $3 \mathrm{~h}$ at room temperature (RT). For washing and rinsing, brains were transferred into the solutions using a $10 \mu \mathrm{l}$ pipette. They were rinsed three times at RT with PBS for 10 min, and afterwards the primary antibody incubation occurred at $4^{\circ} \mathrm{C}$ over night. The incubation solution included 5\% normal goat serum (NGS; Jackson ImmunoResearch), PBS containing 0,3\% Triton X-100 (PBT; Sigma-Aldrich), synapsin antibody, Alexa Fluor 488-coupled phalloidin (Molecular Probes $^{\circledR}$ ), and the according primary antibody (see below). The brains were rinsed three times in PBT for $10 \mathrm{~min}$, followed by incubation with the secondary antibodies (see below) over night at $4^{\circ} \mathrm{C}$. Afterwards, brains were washed three times in PBT for $10 \mathrm{~min}$ and finally embedded in $80 \%$ glycerol. To avoid compression of brains, reinforcing rings were used as spacers (see 2.7). Fixation of cover slide was achieved with nail polish (essence multi dimension, Cosnova $\mathrm{GmbH}$ ).

Primary antibodies and concentrations used: 1:100 of monoclonal primary mouse antibody against synapsin (3C11, \#151101 (13.12.06); Klagges et al., 1996; Utz et al., 2008; Dreyer et al., 2010). 1:10000 of polyclonal antiserum of rabbit against the biogen amin serotonin (5HT; DiaSorin; decribed in Dacks et al., 2006). 1:5000 of rabbit antiserum against neuropeptide Periplaneta americana myoinhibitory peptide (Pea-MIP, GWQDLQGGWamide, described by Predel et al., 2001). 1:200 rabbit antiserum against the catalytic subunit of the protein kinase A (PKAc) of Drosophila melanogaster for specific visualisation of Mushroom Body (Lane and Kalderon, 1993; Farris and Strausfeld, 2003).1:200 of Alexa Fluor 488-coupled phalloidin (Molecular Probes ${ }^{\circledR}$ ).

Secondary antibodies and concentrations used: 1:300 of goat anti-mouse antibodies conjugated to either Cy5 (GAM Cy5) or Cy3 (GAM Cy3) (Jackson ImmunoResearch). 1:300 of goat anti-rabbit antibodies conjugated to either Cy5 (GAR Cy 5) or Cy3 (GAR Cy3) (Jackson ImmunoResearch). Antibodies were chosen to prevent overlapping emission, e.g. GAM Cy5 and GAR Cy3. 


\subsection{Heat shock in water bath}

Embryos of the strain carrying the heat shock construct were reared before and after the heat shock (HS) at $25^{\circ} \mathrm{C}$ to avoid a latent over expression. Heat shock was performed as described by Hillebrand (2010), i.e. 10 minutes in a water basin prewarmed to $46^{\circ} \mathrm{C}$. For this, embryos were transferred into a $15 \mathrm{ml}$ tube $(120 \times 17 \mathrm{~mm}$ Sarstedt). Afterwards, breeding was allowed at different temperatures depending on the experiment. In case of in situ hybridization experiments, rearing was allowed after the $\mathrm{HS}$ at $25^{\circ} \mathrm{C}$ until the respective stage was reached and then the embryos were fixed (chapter 2.5; Posnien et al., 2009). In case of cuticle analysesrearing was allowed either at $25^{\circ} \mathrm{C}$ or at $32^{\circ} \mathrm{C}$ until the cuticle preparation (chapter 2.5 and chapter 3.1.1).

\subsection{Documentation}

In vivo brain scans were documented with a Zeiss LSM 510 and processed using the Zeiss LSM Image Browser (Version 4.2.0.121). Cuticles were documented using a Zeiss Axioplan microscope to record stacks of 10-20 plans. Deconvolution was performed by the "No Neighbour" method of the ImagePro software (Version 6.2; MediaCbernetics). Z-projections were made using the ImageJ (version $1.40 \mathrm{~g}$ ) for cuticle images with the algorithm "maximum intensity" and for in vivo brain projections either with "maximum intensity" or "average intensity".

\subsection{Used computer programs}

NCBI/Blast (http://blast.ncbi.nlm.nih.gov/Blast.cgi), and "Blast against Baylor Data" (www.hgsc.bcm.tmc.edu/blast.hgsc) were used for obtaining and analyzing the regulatory regions of the genes described above. The Operon Oligo Analysis Tool (www.operon.com/ technical/toolkit.aspx) and "OligoCalc: an online oligonucleotide properties calculator" (http:// www.basic.northwestern.edu/biotools/oligocalc.html) were used for primer analysis. The APE-A plasmid Editor (v1.14), was used for sequence analysis and as a computational cloning tool. The MultAlin online tool was used for "Multiple sequence alignment with hierarchical clustering" F. Corpet, 1988 (http://multalin.toulouse.inra.fr/multalin/multalin.html). ImageJ (v 1.43u, 32bit and 64bit) was used for stack analyses and image preparations and Adobe Photoshop CS3 (Version 10.0.1) was used for image and panel preparation. Numbers'09 (Version 2.0.5) was used for stochastical calculations and bar graph illustrations and Pages'09 (Version 4.0.5) for scripting. 


\section{Results and Discussion Part I: Generation of transgenic reporter lines for the analysis of brain development in Tribolium castaneum}

One part of this work was to establish a system to visualize the morphology of the larval brain in Tribolium and to adress questions regarding development. Some transgenic imaging lines already existed and showed distinct signals in the CNS or neural tissue. Those lines originated from the GEKU screen ('Mushroom Body' line; chapter 3.1.4; Trauner et al., 2009), from collaborating laboratories (EFII::DsRedEx; chapter 3.1.4), or from work of former Diploma students in the group of G. Bucher (6xP3::eCFP, Hein; 2007; chapter 3.1.4) and were characterized here. Further lines were to be generated for the visualization of specific cell types of the CNS and brain tissue (chapter 3.1.2). Finally, reporter lines that enabled imaging of subsets of cells in the brain by the expression of reporter genes driven by the regulatory elements of the genes of interest could be generated (chapter 3.1.3). Additionally, transgenic animals for a Tc-six3 overexpression system were generated and characterized.

\subsection{The heat shock $T c$-six 3 overexpression construct is not functional}

By a former student, a construct for production of a ubiquitous Tc-six3 overexpression (piggyBac [3xP3::eGFP; hsp5'UTR, DsRedEx, 3'UTR; hsp5'UTR, ORF Tc-six3, 3'UTR]) was designed. This construct included the Tribolium specific heat shock promoter hsp68 (reviewed in Hillebrand, 2010) driving on the one hand the reporter gene DsRedEx and on the other hand Tc-six3. It was cloned by Julia Schanda and Johannes Schinko (unpublished) and was used here to generate transgenic animals (chapter 2.6). Six lines originating from one $\mathrm{G}_{0}$ were characterized, meaning these lines are siblings.

\subsubsection{Characterization of positive transgenic animals by in situ hybridization and cuticle phenotype}

The functionality of the $T c$-six 3 overexpression heat shock constructs in transgenic animals was tested by different heat shock (HS) experiments. Therefore, embryos of the transgenic lines were collected for $24 \mathrm{~h}$ at $25^{\circ} \mathrm{C}$ and heat shocked for $10 \mathrm{~min}$ at $46^{\circ} \mathrm{C}$. Heat shocked embryos were then kept on lower temperature to avoid further misexpression and hatched after approximately 5 days at $25^{\circ} \mathrm{C}$. Additional heat shocked embryos were kept on $32^{\circ} \mathrm{C}$ to reduce potential self healing processes and larvae hatched after approximately $4 \mathrm{~d}$. In both cases, embryos were prepared in Hoyers Medium for cuticle analysis (chapter 2.5), and in both cases no cuticle defects or malformation caused by a gain of function of Tc-six 3 were detected. 
To confirm the functionality of the construct, whole mount in situ hybridization (chapter 2.4) with heat shocked embryos of these lines was performed. Heat shocks had been done as described above, but embryos were reared for $1 \mathrm{~h}, 2 \mathrm{~h}$, and $3 \mathrm{~h}$ at $25^{\circ} \mathrm{C}$ after $\mathrm{HS}$, followed by fixation. The experiments were repeated several times to collect enough embryos for staining. Due to the small number of eggs received by one collection, the patches were split and fixated. Thus, control embryos (no HS) and embryos fixed $2 \mathrm{~h}$ after HS derived from one egg lay, $1 \mathrm{~h}$ and $3 \mathrm{~h}$ after HS respectively. All patches of heat shocked and non heat shocked embryos were stained simultaneously with the same antisense $T c$-six3 probe. Staining was stopped at the same time for all samples, when staining in the control animals first appeared (Figure 4, A).
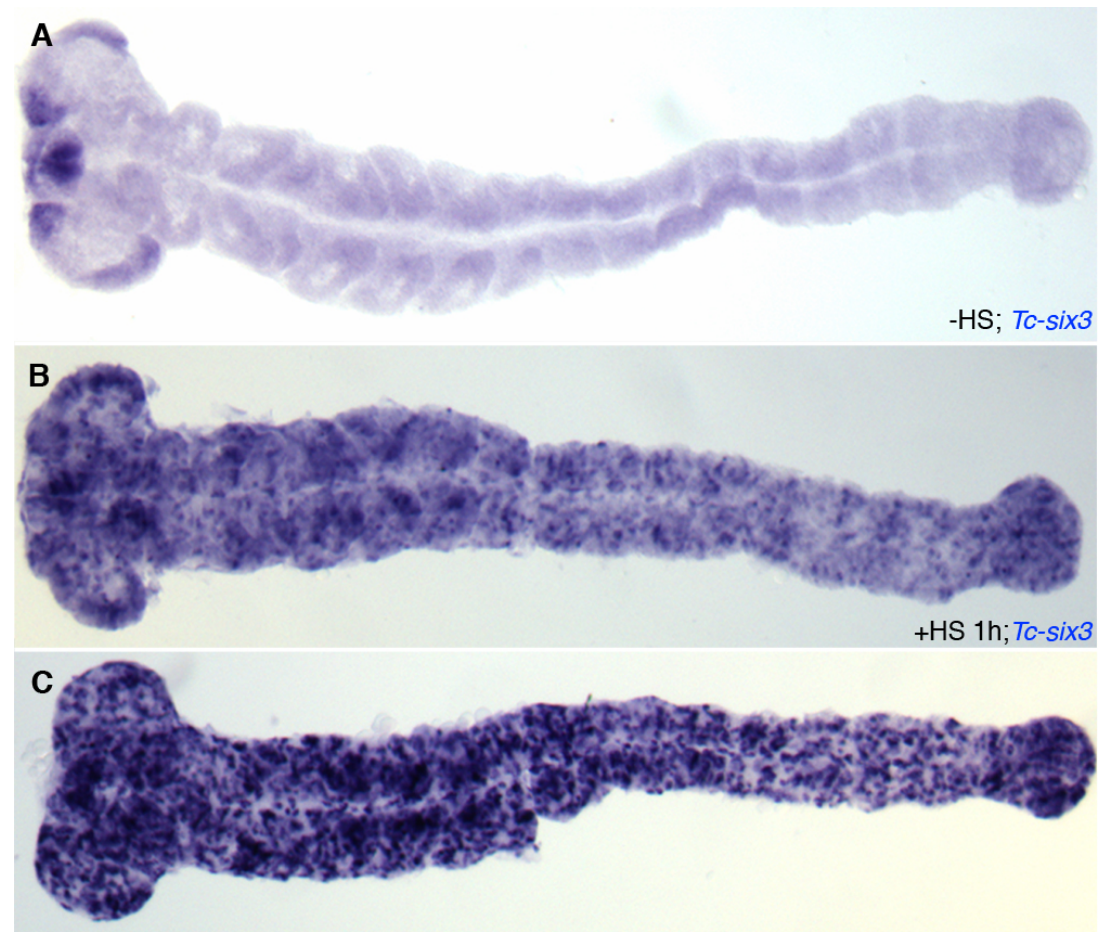

$+\mathrm{HS} 2 \mathrm{~h} ; \mathrm{Tc}-\operatorname{six} 3$

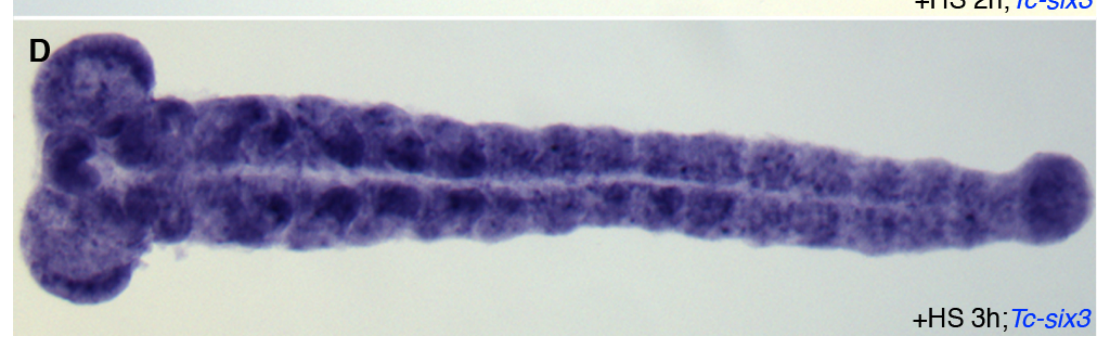

Figure 4: In situ hybridization for $T c$ six3 in the $T c$-six3 overexpression line after heat shock for $10 \mathrm{~min}$ at $46^{\circ} \mathrm{C}$.

(A) elongated germband without heat shock (HS). Expression pattern is WTlike. (B) Expression of Tc-six3 one hour after HS. Staining shows a ubiquitous overexpression. (C) Ubiquitous overxpression $2 \mathrm{~h}$ after HS. (D) Ubiquitous overexpression $3 \mathrm{~h}$ after given HS.

All six lines showed the same expression pattern in all stages (not shown). Representative almost fully elongated embryos of the Tc-six 3 overexpression line 1 are shown in Figure 4. As a negative control, non heat shocked embryos of the transgenic lines were used. These embryos showed an expression pattern which was identical to the native $T c$-six3 expression in all stages (Figure 4, A). In general, in embryos with HS treatment a grainy expression pattern could be detected in all stages. In 
embryos fixed $1 \mathrm{~h}$ after HS, a strong ubiquitous expression pattern of Tc-six 3 could be detected (Figure 4, B). A slight increase of Tc-six3 expression could be detected in embryos fixed $2 \mathrm{~h}$ after HS (Figure 4, C), while embryos fixed $3 \mathrm{~h}$ after HS showed a similar expression pattern compared to the embryos fixed $1 \mathrm{~h}$ after HS (Figure 4, D). This confirmed that the construct was functional for expressing RNA of Tc-six 3 after HS treatment.

Nevertheless, a cuticle phenotype was not detected. This indicated either that overexpression of $T c$ six 3 caused no cuticle phenotype or that no functional Tc-six3 protein was produced. Since no antibody for $T c$-six3 protein detection was available, the construct sequence was analyzed for putative frame shifts or other disturbing properties.

\subsubsection{Genomic DNA analysis reveals errors in construct sequence}

The pre-reporter gene region of the overexpression construct was analyzed by sequencing the respective regions within the genomic DNA (gDNA; Figure 5), isolated from 3 to 5 individuals of each line (chapter 2.3). Sequencing of all Tc-six3 overexpression lines confirmed the linker sequence between the open reading frame (ORF) of Tc-six 3 and the hsp5'UTR to be derived from the PCRII ${ }^{\circledR}$ vector (Figure 5; Appendix Figure 7.2).

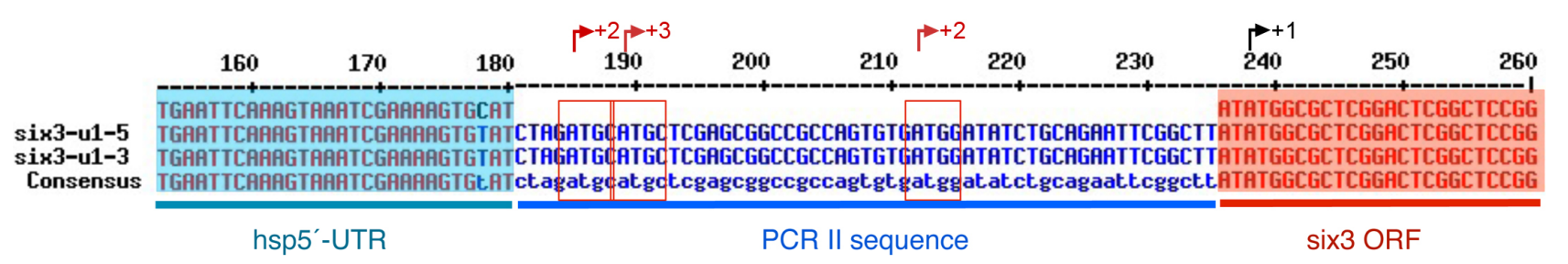

Figure 5: Sequence analysis of the linker between the hsp5 -UTR and the Tc-six3 ORF reveals additional translation start sites.

The hsp5 '-UTR is highlighted in turquoise, the $T c$-six 3 ORF is highlighted in red. Sequences shown here are derived from lines 5 and 3 . The linker sequence of the PCR II ${ }^{\circledR}$ vector is depicted in blue. Within the vector sequence there are three putative translation start sites (marked by red square), causing frame-shifts. The black arrow marks the original translation start site of $T c-s i x 3$.

In Figure 5, lines 3 and 5 are shown as representatives for all 6 hs-Tc-six3 overexpression lines. The endogenous translation start site (TLS) of Tc-six3 was present and unchanged (indicated by a black arrow, Figure 5). Within the linker sequence, several additional translation start sites (red squares, Figure 5) were found. The first TLS causing a frame shift of 1 base pair was coding for a short protein of 9 amino acids (AA) until the first stop codon. The second TLS caused a frame shift of 2 
bp leading to a protein of 7 AA length. Both ORFs originating from the first TLSs stop before the Tc-six3 ATG. The third TLS caused a frame shift of $1 \mathrm{bp}$ and lead to a protein sequence of 17 AA. Here the coding sequence offerlapped with the ORF of Tc-six3.

Potentially, these additional TLSs disturbed the translation of Tc-six 3 mRNA. Therefore the total amount of functional Tc-six 3 protein after HS treatment was possibly too low to cause any cuticle phenotypes. To confirm the presence of ubiquitous Tc-six3 protein after HS an immunohistochemistry staining with a respective antibody against $T c$-six 3 would be necessary.

\subsection{Generation of reporter lines regulated by the native upstream regions of $T c$-elav and $T c$ - repo}

Marker lines showing reporter gene expression in specific tissues of the CNS were to be generated. Such lines can then be used for analysis of wildtype brains as well as for studies of loss or gain of function of various genes. The two genes Tc-elav and Tc-repo, were selected to generate such reporter lines. Homologues of Tc-elav were known to be expressed pan-neurally (Yannoni and White, 1997; Berger et al., 2007). Furthermore Tc-elav expression was also shown for Tribolium by whole mount in situ hybridization (Simonnet, personal communication). Tc-repo was chosen since homologous genes in other species are specifically expressed in glial cells arising from neuroectoderm (Yuasa et al., 2003). To establish the desired reporter lines, constructs including the putative regulatory regions of Tc-elav and Tc-repo in combination with the endogenous promoters driving various reporter genes were designed.

\subsubsection{The transgenic line for the regulatory region of $T c$-elav revealed a muscle expression pattern}

Before cloning, RACE-PCR was performed to identify the 5'UTR of Tc-elav (chapter 2.2), which was also important for the identification of the endogenous promoter.

A 5'UTR of a total length of 409 bp could be identified. Interestingly, by comparison to gDNA, a $1586 \mathrm{bp}$ long intron was found. This intron splits the 5'UTR into two fragments with $182 \mathrm{bp}$ at the 5'end and $227 \mathrm{bp}$ at the 3'end close to the translation start site ATG (Appendix Figure 7.1). For cloning the regulatory region, the upstream region of Tc-elav starting $28 \mathrm{bp}$ and ending $2579 \mathrm{bp}$ upstream of the translation start was cloned. Thus, the identified intron was included. The total length of this fragment was limited by a gene prediction for the Glean_4647, which lies on the negative strand and codes for a zinc carboxypeptidase domain. The $400 \mathrm{bp}$ upstream of the gene prediction Glean_4647 were omitted to be included into the regulatory region of Tc-elav (reg. Tc- 
elav). Thus, the total length of reg. Tc-elav was $2551 \mathrm{bp}$. This fragment was used for driving the reporter gene RFP with a membrane tag (mRFP). Transgenesis (chapter 2.6) using the construct 'reg. Tc-elav::mRFP' (Appendix Figure 7.3.1), yielded two transgenic lines from two independent $\mathrm{G}_{0}$.

When analyzing the first larval instar (L1), no fluorescent signal was detected in $T c-v w$ larva (Figure 6, A), while the larvae of both 'reg. Tc-elav::mRFP' lines showed an identical expression pattern for mRFP. The expression was detected exclusively within the muscles (Figure 6, B). The brain is embedded by muscles positive for the mRFP signal (Figure 6, C). This was shown by crossing experiments with the 6xP3::eCFP line, which marks glial cells (chapter 1.4.2; for better contrast depicted in green). Due to the muscle expression pattern, no further investigations of the Tc-elav lines were performed.
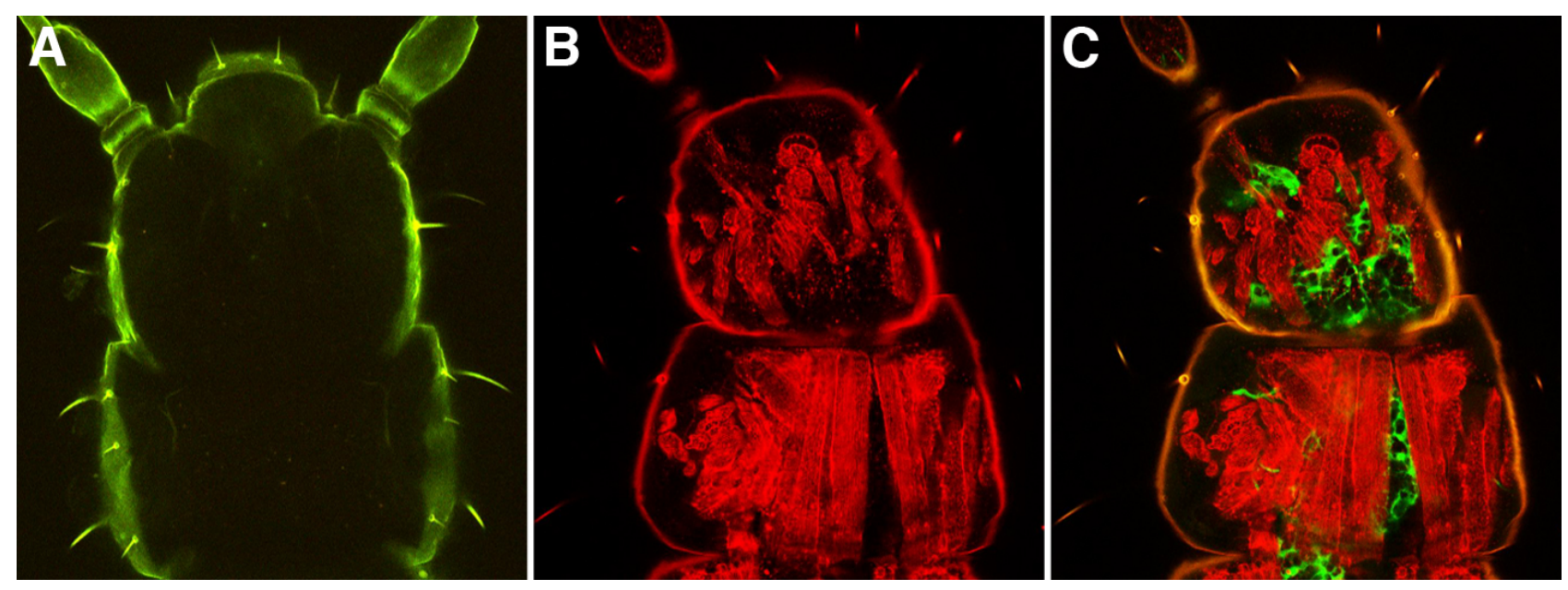

Figure 6: The line 'reg. Tc-elav::mRFP' drives reporter gene expression in muscles.

(A) The first instar larva (L1) of the vw strain shows an autofluorescence of the cuticle, shown as negative control. A larva derived from crossing of the lines $6 \times \mathrm{XP} 3:$ eCFP and 'reg. Tc-elav::mRFP' is depicted in panel (B) and (C). (B) Muscular expression pattern of mRFP under the control of regulatory region of Tc-elav, only the red channel. (C) The eCFP signal is depicted in green. Brain tissue is visible under the muscles in the head capsule. Anterior is facing up.

Taken together, two independent lines whith similar expression patterns in the muscles were created. Due to the very similar expression pattern it is not very likely, that enhancer elements surrounding the insertion locus influence the expression pattern. However, to exclude a insertion locus dependent influence an inverse PCR on the gDNA of individuals of both lines could be performed. Due to the independent generation of such lines it is unlikely, that integration occured independently twice at the same genomic locus. Therefore it is suggested, that elements included within the cloned regulatory regions specifically cause this expression pattern. 
For example, the 'reg. of Tc-elav' could include sequences like so called insulators. It is known from other species like Dorosphila, that such elements are able to shield promoters from nearby enhancer or silencer elements (Brasset and Vaury, 2005; Bushey et al., 2008). The utilization of such elements to create transgenic reporter lines in Tribolium castaneum would facilitate the identification of enhancer elements including in putative regulatory sequences. That means, the expression pattern of reporter lines included such insulators would be due to the enhancers within the construct. Since locus specific integration was not feasible in Tribolium at that time, using insulators would display an alternative option to achieve specific expression.

\subsubsection{The transgenic line for the regulatory region of Tc-repo shows a glial expression pattern}

Genomic DNA of the SB strain was used to amplify a fragment upstream of Tc-repo with a total length of $4510 \mathrm{bp}$. Tc-repo possesses a $86 \mathrm{bp}$ long 5'UTR with a TATA-box at -36 (relative to the transcription start site). These elements were included within the fragment. In order to select optimal primers, $44 \mathrm{bp}$ of the 5'UTR were not included.

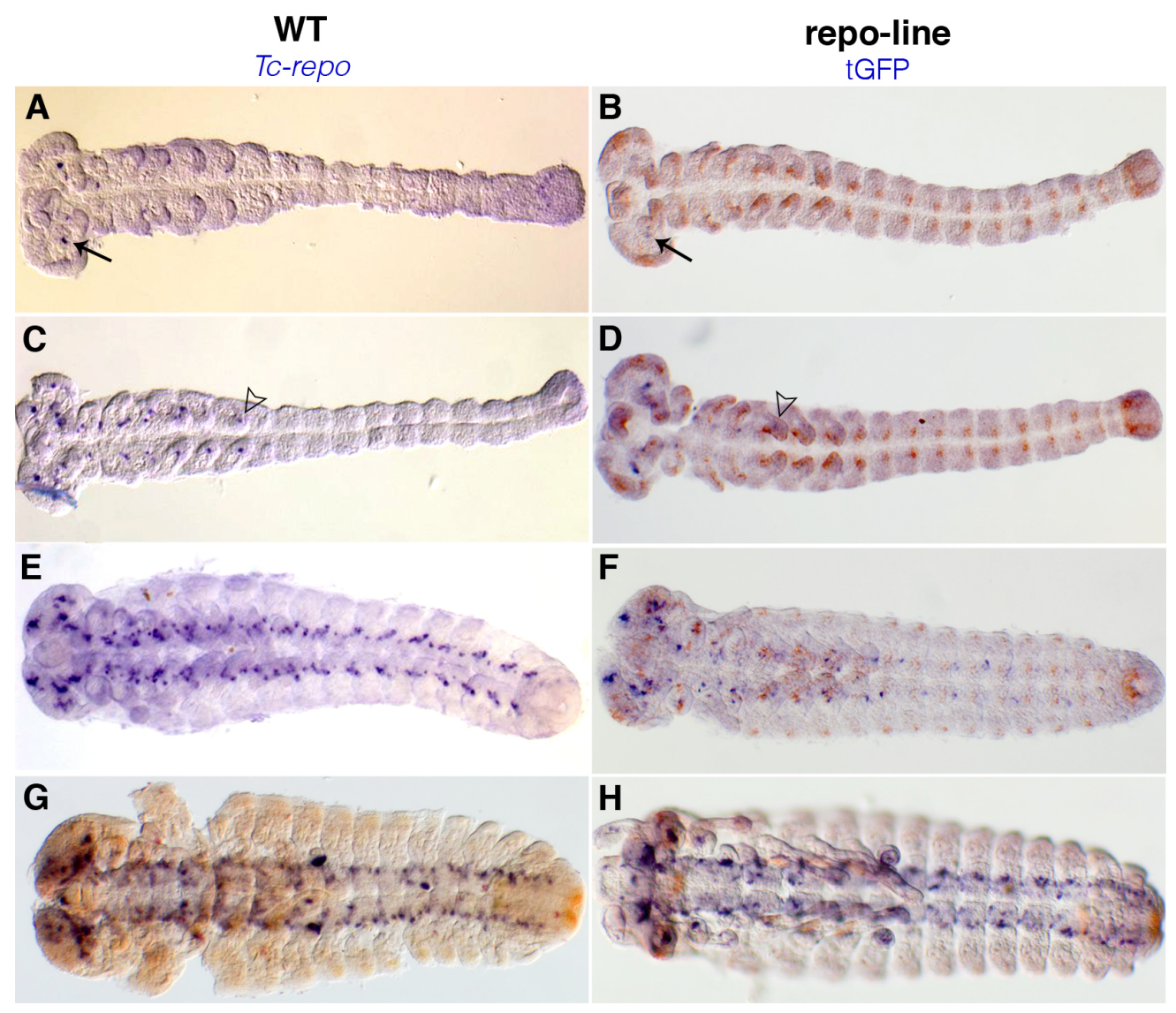

Figure 7: The reporter gene expression pattern of the line 'reg. $T c$ repo::tGFP' mimics the endogenous Tc-repo expression in WTembryos with delay.

The expression pattern of Tc-repo is depicted in the WT-embryos (A, $\mathrm{C}, \mathrm{E}, \mathrm{G})$ in blue. The tGFP expression pattern under the control of 'reg. Tcrepo' $(\mathrm{B}, \mathrm{D}, \mathrm{F}, \mathrm{H})$ is also depicted in blue. Embryos in the panels (B, D, F, G, H) show a double staining with Tc-wg, shown in red. In embryos of the transgenic line 'reg. $T c$ repo::tGFP', the reporter gene expression pattern is similar to the endogenous Tc-repo expression in WT-embryos. Arrows (A, B) and arrowheads (C, D) indicate similar expression domains in embryos of differing ages. Thus, tGFP expression in the transgenic line is delayed. Anterior is orientated to the left. 
The regulatory region of Tc-repo (reg. Tc-repo) was used to drive the reporter gene turboGFP (tGFP; Appendix Figure 7.3.2). Transgenesis resulted in transgenic offspring of one $\mathrm{G}_{0}$. This line was analyzed for tGFP expression. In situ hybridization experiments were performed to compare endogenous Tc-repo expression in WT embryos with tGFP expression of the reporter line 'reg. Tcrepo::tGFP'. Transgenic embryos were stained with an antisense probe for tGFP and SB-embryos were incubated with an antisense probe for Tc-repo.

The endogenous expression of Tc-repo mRNA was detected first in two points in the head of an elongating embryo (black arrow; Figure 7, A). With ongoing development, a segmental expression pattern arose from anterior to posterior (Figure 7, C and E), lateral to the midline in parallel rows. The expression pattern in the head was very dynamic. In the labrum and the area around the stomodaeum no Tc-repo could be detected, initially(Figure 7, E), but was detectable in later stages (Figure 7, G). Testing the expression pattern of the reporter gene tGFP in the transgenic animals for the construct 'reg. Tc-repo::tGFP', a delayed expression pattern was detected, however very similar to the endogenous expression of Tc-repo. The two domains in the head were detectable at a fully elongated stage (black arrow; Figure 7, B). Segmental patterning arose in embryos being close to retracting movements (arrowhead; Figure 7, D and F). Taken together, even though delayed, the expression pattern of the reporter gene was similar to the endogenous Tc-repo expression.

First instar (L1) larvae of the transgenic line 'reg. Tc-repo::tGFP' were scanned for tGFP expression in the head. The tGFP signal was detectable in neural tissue. The expression within the soma of cells positive for the tGFP signal was visible as dots within the brain (open white arrowheads; Figure 8, C). Apparently, tGFP was located in the cell lumen, recognizable by faint lines spreading out from soma (Figure 8, C). A prominent expression domain (open white arrow; Figure 8, C) was present anterior lateral to the stomatogastric nervous system (STNS), which showed a tGFP signal as well (Figure 8, C). Starting from these lateral domains, two symmetric major projections were leading into the labrum and into the antenna. The tGFP positive cells projecting into the antenna were forming a tube (Figure $8, \mathrm{C}^{\prime}$ ).

Comparing the signals of the line 6xP3::DsRedEx (Figure 8, B and B') with the reporter line 'reg. Tc-repo::tGFP' in the antenna, it appeared that these signals are not overlapping. Rather, the cells positive for tGFP enclosed the bundles positive for DsRedEx (Figure 8, B' and C'). Since the line 6xP3::DsRedEx marks a subset of glial cells in the brain (chapter 1.4.2), it is very likely, that the transgenic line 'reg. Tc-repo::tGFP' marks an additional and non overlapping subset. It is known from Drosophila, that all glial cells derived from the neuroectoderm are positive for the transcription factor repo (reviewed in Edwards and Meinertzhagen, 2010). Furthermore the repo 
positive cells are distinguishable into three types regarding their location, surface, neuropile, and cortex glia (Awasaki et al., 2008; Edwards and Meinertzhagen, 2010). Due to the position of the 6xP3::DsRedEx positive cells, it is suggested that these cells are cortex and neuropile glia. While cortex glia form a mesh in the cortex of the CNS, the neuropile glia enwrap the surface of neuropiles. Thus, these types of glia are embedded in the brain, as it is detectable in the case of the transgenic reporter line 6xP3::DsREdEx. In contrast the reporter gene signals of the 'reg. Tcrepo::tGFP' line seem to form a superficial ensheathing layer, which is once detectable in the antenna as well as in the case of the STNS (compare Figure 8, B', $\mathrm{C}^{\prime}$ and B, C). Thus it is suggested, that the line 'reg. Tc-repo::tGFP' marks a subset of surface glia.
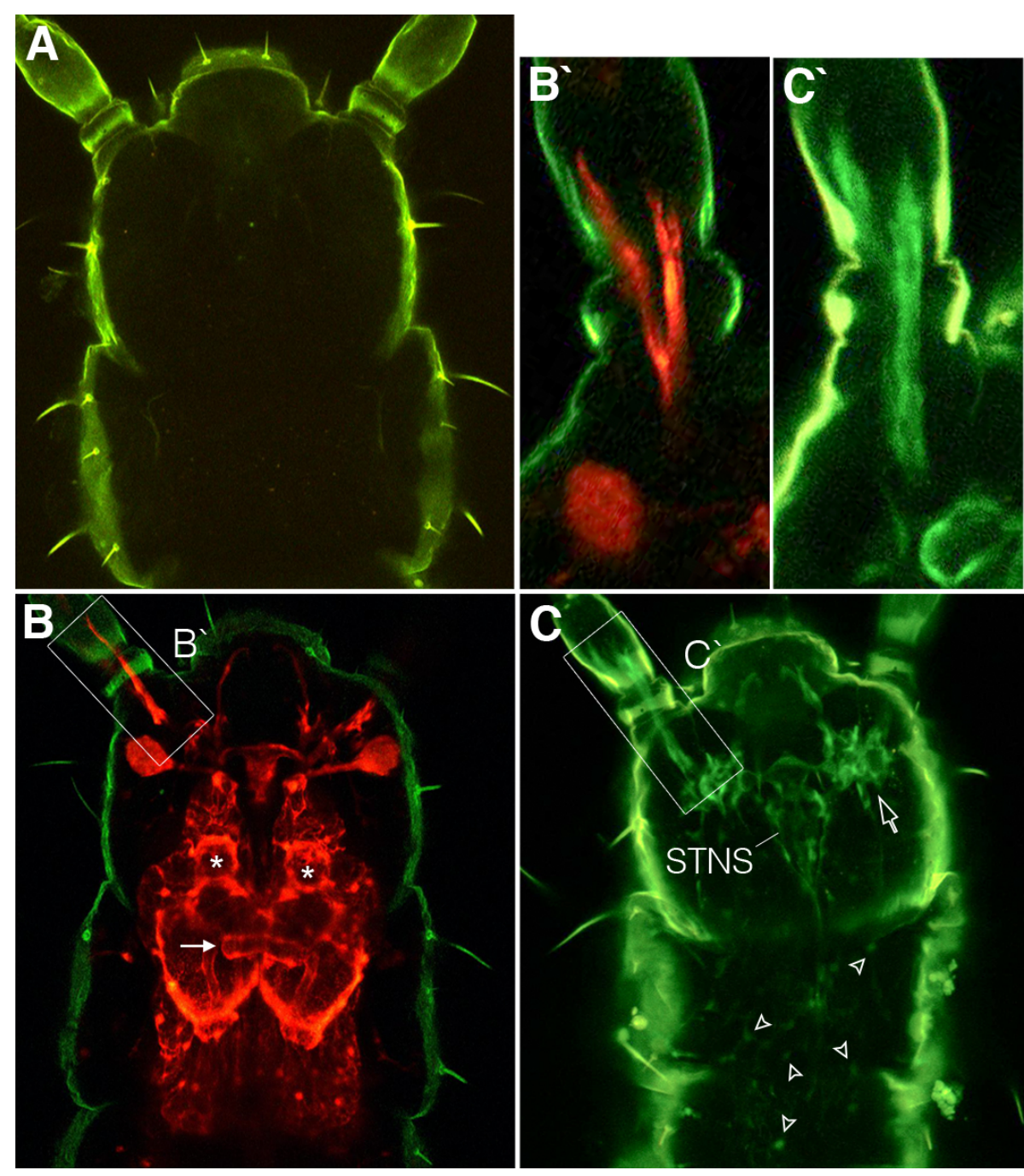

Figure 8: Expression analysis of tGFP in L1 larva of the line 'reg. Tc-repo::tGFP'.

(A) The larva of the vw strain shows autofluorescence of the cuticle only. (B) Expression pattern in L1 larva transgenic for $6 \times \mathrm{P} 3:$ :DsRedEx, which marks glial cells in red (see chapter 1.4 .2 and 3.4 for details). Antennal Lobes are marked by white asterisks; the Central Body is pointed out by a white arrow. (C) L1 larva of the line 'reg. Tc-repo::tGFP', shown in green. tGFP positive cells in the brain are pointed out by white open arrowheads. The antennal nerve projects into a structure positive for tGFP expression, indicated by the open white arrow.The stomatogastric nervous system (STNS) is also marked by tGFP expression. Enlarged antennal sections (B', C') are marked with a white square in the according image (B, C). The line 'reg. Tc-repo::tGFP' probably marks a subset of surface glia. Anterior is facing up. 
To prove these hypotheses, colocalization experiments were performed by crossing the 'reg. $T c$ repo::tGFP' line with the 6xP3::DsRedEx line. However, a full separation of signals was not possible with the available filter sets. For a detailed analysis of colocalization either antibodies against specific markers could be used, e.g. against Tc-repo as a glia marker (Halter et al., 1995) or anti DsRedEx and tGFP themselves in a crossed background as described above. Thereby it could be verified, if the transgenic lines mark additive subtypes of glia.

Taken together, the embryonal expression pattern of tGFP mRNA is similar to the endogenous expression pattern of Tc-repo. The tGFP signal present in cells of the neural tissue and its surroundings in larval brains, makes it very likely that the line 'reg. Tc-repo::tGFP' marks glial cells. In comparison to the glial marking line 6xP3::DsRedEx, it is very likely that both lines mark different subtypes of glia, while 6xP3::DsRedEx appears to mark cortex and neuropile glia, the line 'reg. Tc-repo::tGFP' appears to drive expression in surface glia. However, it also became apparent that the line $6 \mathrm{xP} 3:$ :DsRedEx provides a better marker for brain morphology. Therefore, further analyses of the line 'reg. Tc-repo::tGFP' were not performed.

\subsection{Generation of $T c-s i x 3$ and $T c-r x$ reporter lines}

In order to analyze the regulation of the identity of neural lineages, lines where a reporter gene is driven under the control of the endogenous regulatory region and a promoter of a gene expressed in a very restricted set of cells in the brain were needed. Such lines should enable tracing the development starting from NBs to distinct regions or even neuropiles in the larval brain. For this, the two genes $T c-\operatorname{six} 3$ and $T c-r x$ were chosen. The reasons for selecting these genes are: 1) Both genes have an exclusive anterior most expression within the embryo, which stays restricted to the head during development. Hence, they are specific to the brain. 2) From other models it is known, that correlating regions positive for these genes are forming parts of the brain and in the case of six 3 of the Central Complex (Boyan and Williams 1997, de Velasco et al., 2007). 3) The expression domains are small and only a small number of NBs is positive for their expression, which alleviates the tracing from NB to neuropile in the larval brain.

\subsubsection{Identification of $T c$-six 3 and $T c-r x$ positive NBs in the anterior neuroectoderm}

For analyzing the amount of NBs which were positive for $T c-s i x 3$ or $T c-r x$ expression within the neuroectoderm, 6h staged egg lays were collected at $32^{\circ} \mathrm{C}: 12-18 \mathrm{~h}, 18-24 \mathrm{~h}$, and $24-30 \mathrm{~h}$. This enabled estimation of the age of the according embryos. By double in situ hybridizations with the NB marker Tc-asense (Tc-ase; Wheeler et al., 2003) and Tc-six3 or Tc-rx, the amount of NBs, which 
arose in the respective gene expression region, was quantified. From Drosophila, it is known that asense remains expressed in delaminating NBs at least until the first division of the neural precursor (Brand et al., 1993).

A

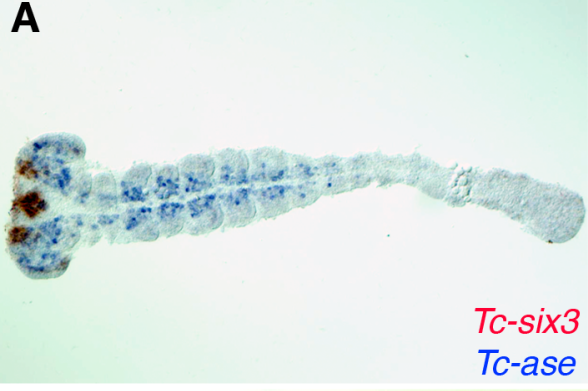

B
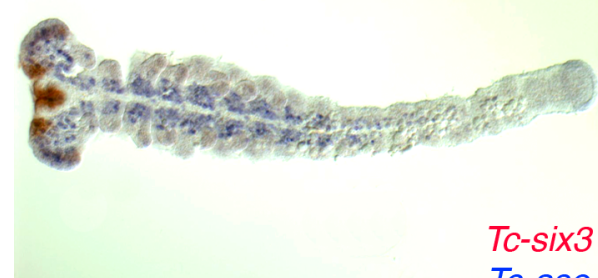

C
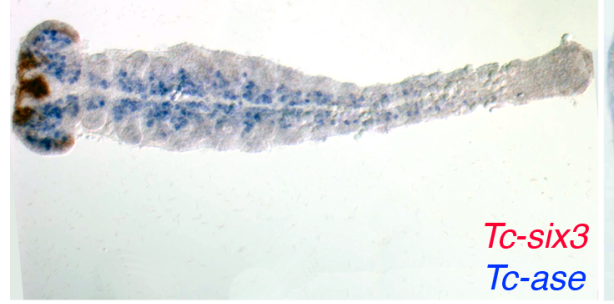

D

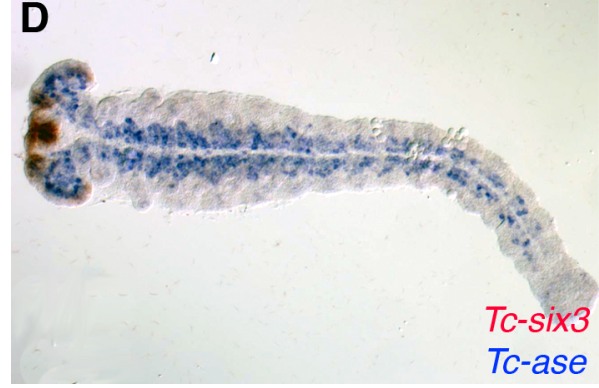

\section{A}

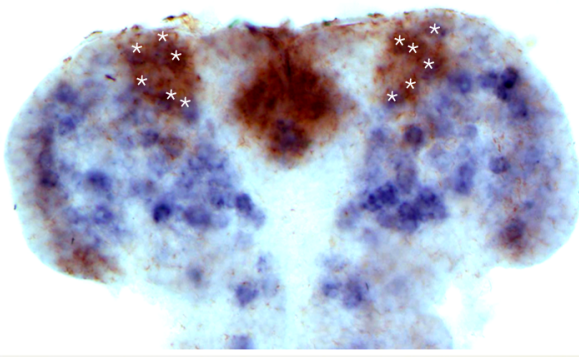

B

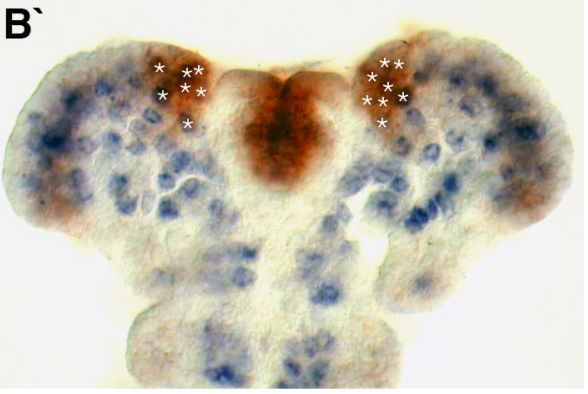

C

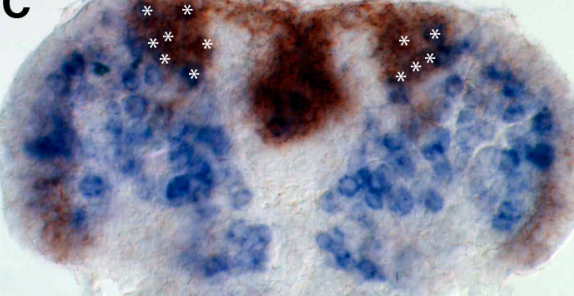

E
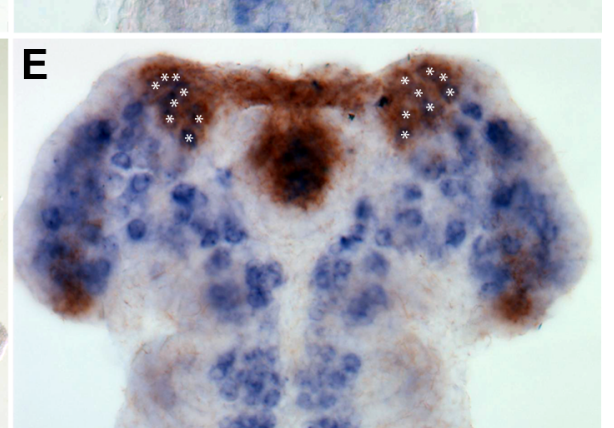

Figure 9: At least $8 \mathrm{NBs}$ delaminate from the Tc-six 3 positive region.

(A-D) WT embryos at different developmental stages with double in situ hybridization for $T c$-six3 (red) and the neuroblast marker Tc-ase (blue). (A) An embryo approximately $14 \mathrm{~h}$ old. (B) An embryo approximately 16h old. (C) An embryo approximately $18 \mathrm{~h}$ old. (D) An embryo approximately $24 \mathrm{~h}$ old. (E) The head of an embryo of the approximately same age as the embryo depicted in D. Asterisks indicate NBs positive for $T c$ six 3 expression. At an age of approximately 14h, $6 \mathrm{NBs}$ in the region of $T c$-six 3 arose up to a maximum amount of $8 \mathrm{NBs}$ at 24h. Indicated ages correlate to the development at $32^{\circ} \mathrm{C}$.

By staged egg lays, a clear number and temporal series of arising NBs from the $T c$-six3 region was to be elucidated. The expression pattern of $T c$-ase started at approximately $12 \mathrm{~h}$ at $32^{\circ} \mathrm{C}$. The first NB positive for $T c$-six 3 is the most posterior within the expression domain median lateral to the labrum. The numbers of NBs varied within same aged embryos and even per hemisphere (compare hemispheres in Figure 9, B` and C). In some cases, young embryos about $14 \mathrm{~h}$ old at $32^{\circ} \mathrm{C}$ had more Tc-ase marked NBs within the Tc-six3 region than older ones (compare Figure 9, A and C). Analysis of embryos older than $24 \mathrm{~h}$ was not possible using this method, since technical limitation occurs 
through the need to flatten the head without disturbing brain morphology and on the other hand because of advancing head morphology. In both cases the neural tissue separates from the ectoderm - meaning that the NBs are shifted relative to the ectoderm and cannot be assigned unequivocally. Taken together, up to $8 \mathrm{NBs}$ positive for Tc-six3 were detected in the neuroectoderm at an age of $24 \mathrm{~h}$ at $32^{\circ} \mathrm{C}$ (Figure 9, D and E). However, it cannot be excluded that more NBs delaminate subsequently.
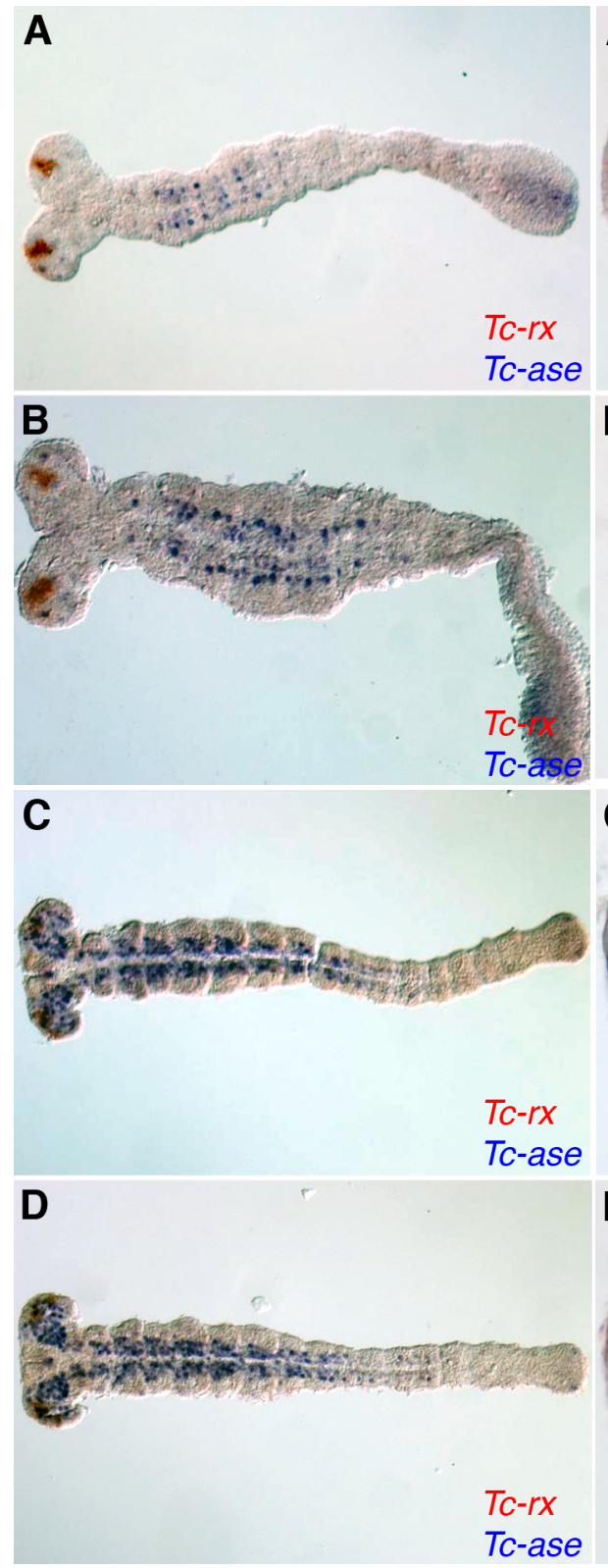
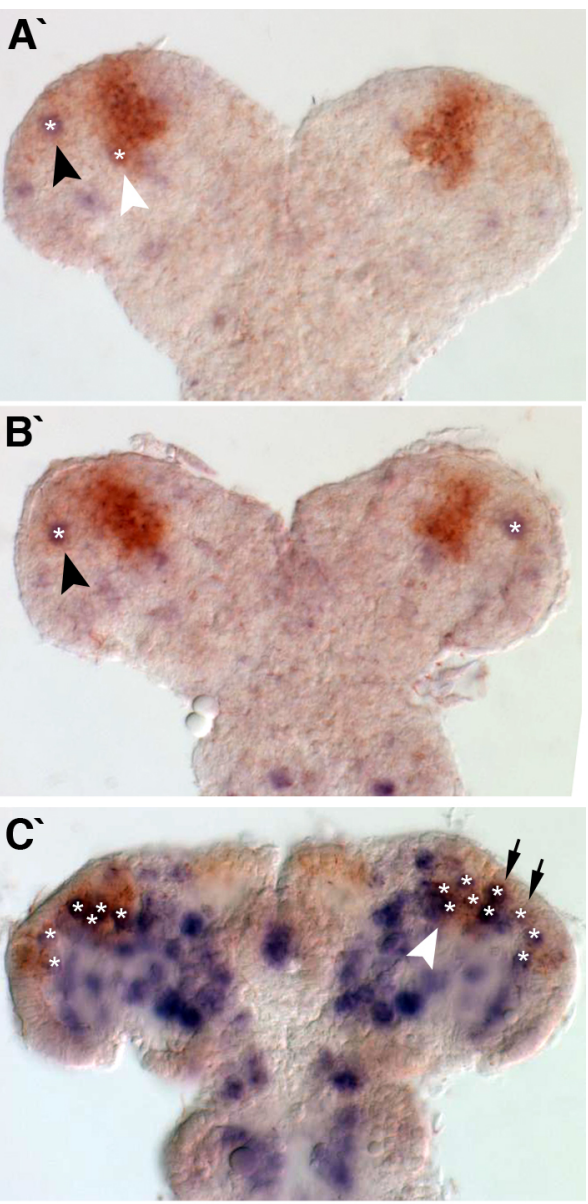

\section{D'}

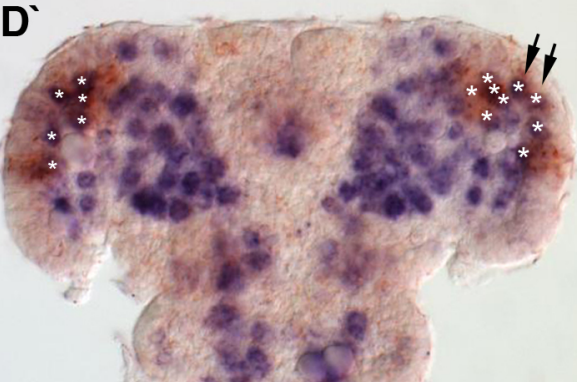

Figure 10: At least 9 NBs delaminate from the $T c-r x$ positive region.

(A-D) WT embryos at different developmental stages with double in situ hybridization for $T c-r x$ (red) and the neuroblast marker Tc-ase (blue). The $T c$ ase expression pattern is detectable in elongating embryos, approximately $12 \mathrm{~h}$ old (A). (C, D) The oldest embryo with an age of approximately $18 \mathrm{~h}$. Asterisks indicate NBs positive for $T c-r x$ expression. The first NBs arise in lateral part of $T c-r x$ region at approximately $12 \mathrm{~h}$ (black arrowhead A', B'). A group of NBs arise in the central domain, indicated by white a arrowhead $\left(C^{\prime}\right)$. In the lateral domain, 2 additional NBs arise in at an age of $18 \mathrm{~h}$, indicated by black arrows (D'). Thus, at least 9 NBs delaminate up to an age of $18 \mathrm{~h}$. Indicated ages correlate to the development at $32^{\circ} \mathrm{C}$.

Double in situ analysis of Tc-ase and Tc-rx was performed to identify the amount of NBs in the $T c$ $r x$ positive region. The first NB positive for $T c-r x$ was detected in approximately $12 \mathrm{~h}$ old embryos within the lateral domain at the rim of the head lobes (black arrowhead, Figure 10, A', B'). The next group of NBs arose in the central domain with 2-3 NBs at the same time (white arrowhead, Figure 
10, C'). In the lateral rim domain of the Tc-rx positive region, only two further NBs were detected in up to $18 \mathrm{~h}$ old embryos (black arrows, Figure 10, $\mathrm{C}^{\prime}$ and $\mathrm{D}^{\prime}$ ). Compared to the NB amount positive for Tc-six3, a variation of the NB number also occurred, both per hemisphere and in same aged embryos (Figure 10, C, D). Nevertheless, a maximum number of 9 NBs arising in the Tc-rx region could be detected up to an age of $18 \mathrm{~h}$ at $32^{\circ} \mathrm{C}$. Also here, it is unclear whether more NBs delaminate at later stages.

The variation of the NB amount among the different ages was remarkable. There are numerous possible reasons, for example that there is no distinct temporal pattern for arising NBs. However, the preparation technique could be another reason, meaning that NBs got out of place by flattening the embryo's head. A difference in the NB amount between hemispheres and stages was also detected in embryos which had not been flattened, but left in natural shape for analysis. However, it is known that NBs delaminate from the neuroectoderm. This was also observed in older stages, where a correlation between the ectodermal surface layer and the neural tissue below was not given anymore. This indicates that in this case colocalization experiments by in situ hybridizations are only partially efficient to appoint the total amount of $T c$-six3 and $T c-r x$ positive NBs by light microscopy. In this case, antibody staining against the neuroblast marker $T c-A S E$ and either Tc-Six3 or $T c-R x$ should be performed. Simultaneous detection of these proteins within NBs would elucidate a definitive number. Nevertheless, at least $8 \mathrm{NBs}$ positive for $T c$-six 3 were detected up to an age of 24h, and 9 NB positive for Tc-rx up to an age of $18 \mathrm{~h}$.

\subsection{2. $T c-\operatorname{six} 3$ reporter lines}

Reporter lines expressing a reporter gene in the same pattern as a gene of interest were to be generated. The endogenous promoter and putative regulatory regions of Tc-six 3 were used to drive expression of the reporter gene. For this, the endogenous promoter of Tc-six3 was analyzed first. By two independent RACE-PCR experiments (chapter 2.2) the 5'UTRs of Tc-six3 were identified. Before sequences of regulatory regions were amplified by PCR of gDNA, the respective regions were analyzed for gene predictions or repetitive sequences (Figure 11, Panel B). Since the enhancer elements, which were driving the endogenous Tc-six3 expression, were unknown, several regions with approximately $5 \mathrm{~kb}$ length were cloned with an overlap of approximately $500 \mathrm{bp}$. These fragments were combined with the endogenous promoter of Tc-six 3 to drive the reporter gene tGFP (see Appendix 7.3.3. - 7.3.6.). The constructs were used to create transgenic lines, which were subsequently analyzed regarding their expression pattern. 


\subsubsection{Generation of $T c$-six 3 reporter lines}

In the case of Tc-six3, two variants of 5'UTRs with two different transcription start sites (TSS) were detected. The longer 5'UTR sequence was 96 bp long. This sequence starts with TSS 1 (highlighted pink, Figure 11, Panel A), which is embedded in a consensus sequence of a putative TFIIB Recognition Element (BRE, Lagrarange et al., 1998; Butler and Kadonaga, 2002). A BRE usually lies upstream of a TATA box, which was not given here. Therefore, it is probably not functional. However, this element had the expected distance of $30 \mathrm{bp}$ to the second TSS. The latter led to a 5'UTR with a length of 66 bp. This TSS 2 (highlighted blue, Figure 11, Panel A) had a typical consensus sequence for TSS, which is also called Initiator element (reviewed in Butler and Kadonaga, 2002). No further core promoter elements were found.

The fragment called 'Tc-six3 5up' is shorter than $5 \mathrm{~kb}$, it includes the entire 5'UTR starting from -1 and ending at -4133 upstream of the translation start site (TLS) of Tc-six3 (purple line; Figure 11, Panel B). This construct already included the endogenous promoter sequence of the gene Tc-six3, while all other putative regulatory regions had to be fused to such a promoter.

The basal 'Tc-six3 promoter element' was characterized carefully (short line under 'Tc-six3 5up' construct; Figure 11, Panel B), because it was used for all fragments. By analyzing the upstream region, 106 bp upstream of the TLS of Tc-six3, the gene prediction Glean_1196 was detected. This gene prediction has a total length of $225 \mathrm{bp}$ and lies on the complementary strand. The 'Tc-six3 promoter element' includes the entire 5'UTR and has a total size of $657 \mathrm{bp}$. Thus, also the glean prediction is included in the 'Tc-six3 promoter element' (short line under 'Tc-six3 5up' construct; Figure 11, Panel B). The sequencing analyses of the finished constructs revealed two shortcomings. First, a deletion within the construct 'Tc-six3 promoter element' was identified. 499 bp upstream of the TLS of the reporter gene tGFP, 9 bp (TGT GAT ACA) are deleted (Figure 11, Panel C). The construct 'Tc-six3 5up', which was amplified in one piece, has no deletion (Figure 11, Panel C). Second, the reverse primer for the endogenous promoter sequence as well as the 'Tc-six3 5up' construct include an additional translation start site of $18 \mathrm{bp}$ upstream of the original ATG of the reporter gene tGFP. This does not lead to a frame shift, but to additional 9 amino acids at the $\mathrm{N}$ terminus of tGFP.

For the upstream and downstream sequences, primers were designed outside of repetitive

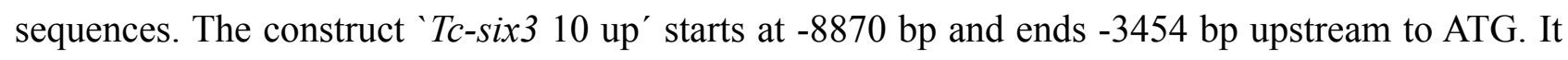
has a total size of $5416 \mathrm{bp}$ and an overlap with the 'Tc-six3 5up' construct of 679 bp (blue line; Figure 11, Panel B). 


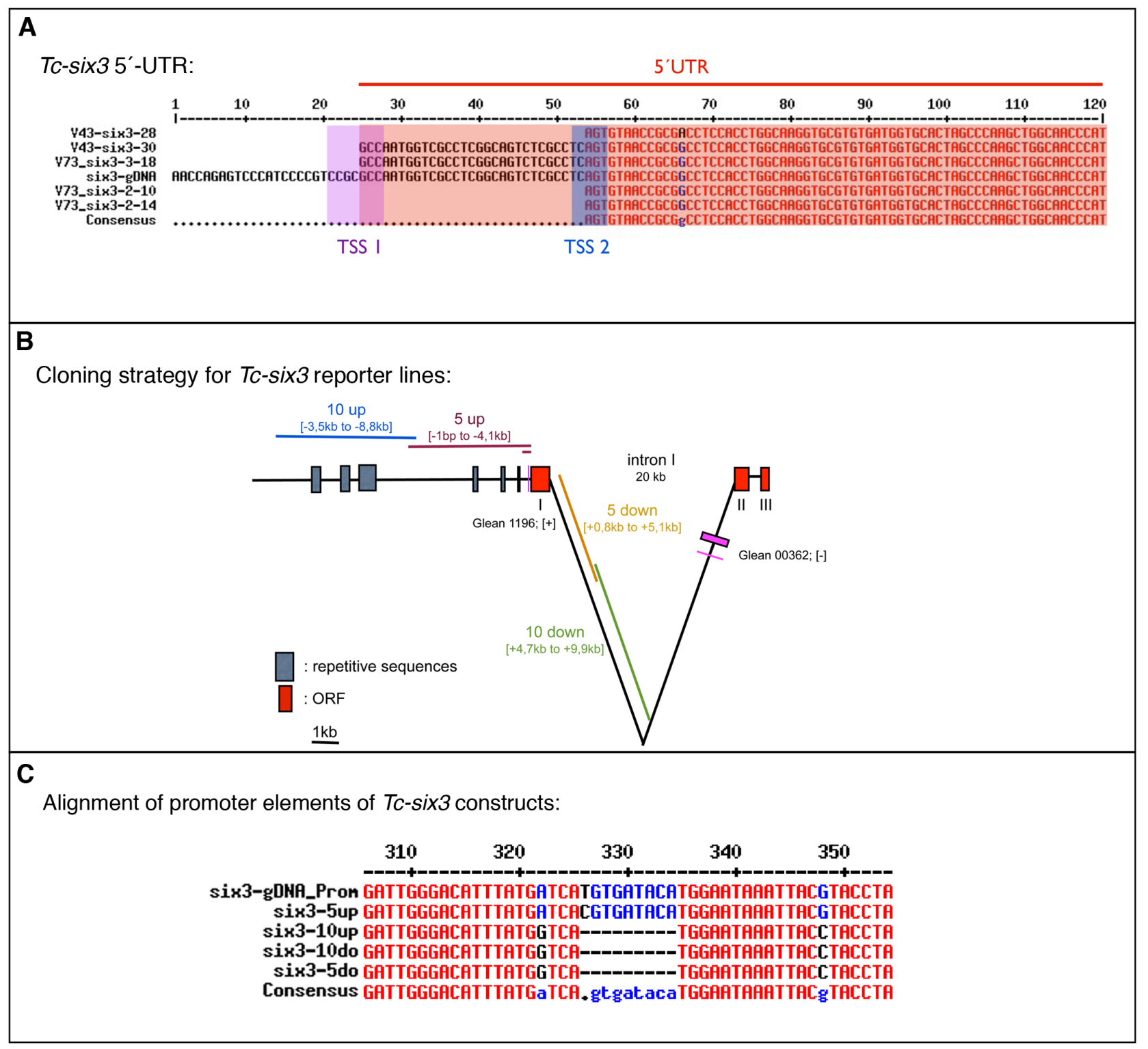

Figure 11: 5 'UTR and cloning strategy for the transgenic reporter lines of $T c$-six3

(A) Within the 5'UTR of $T c$-six 3 (highlighted red) there are two different Transcription Start Sites (TSSs). TSS 1 (highlighted pink) was verified by two independent RACE PCR experiments and lead to a 5'UTR length of $96 \mathrm{bp}$. TSS 2 (highlighted blue) represents the typical consensus sequence with an 5'UTR length 66 of bp. (B) Schematic view of the genomic sequences, which were chosen for reporter gene constructs. A 657 bp long 'Tc-six 3 promoter element' was cloned, including the entire 5'UTR (depicted as a short line under the 5 up construct). All sequences were designed to result in an overlap of at least $500 \mathrm{bp}$ with adjacent regions. These regulatory regions were combined with the endogenous basal promoter driving tGFP, except for constructs 5up which already included the basal promoter. The downstream regulatory regions were cloned in front of the basal promoter. The generated lines with the construct ' $T c$ six3 10up' (highlighted in blue) were positively tested for in situ and partially in vivo expression of tGFP. In lines carrying the constructs 'Tc-six 35 up' and 'Tc-six 35 down', the reporter gene expression was detected only in in situ hybridization experiments. No transgenic line was generated with the construct 'Tc-six3 10down'. (C) Alignment of sequenced promoter regions of injected Tc-six 3 reporter constructs. High consensus sequences are depicted in red. Low consensus sequences are depicted in blue. Reporter constructs six3-10up, 10down, and 5down have a deletion of TGTGATACA, due to a deletion in the 'Tc-six3 promoter element' construct (see chapter 2.3). Repetitive sequence regions are depicted as gray boxes; ORFs are depicted as red boxes; predicted gleans are depicted as pink boxes. 
The constructs Tc-six3 'down' are located in the first intron of Tc-six3, which has a size of 19116 bp. At position +15254 bp downstream of the TLS of Tc-six3, the gene prediction Glean_362 starts on the sense strand. The sequences for reporter line constructs were chosen not to overlap with this gene prediction. Thus, the construct 'Tc-six 3 down' starts at +767 and ends +5140 bp downstream of the TLS of Tc-six3 (yellow line; Figure 11, Panel B). It has a total size of $4373 \mathrm{bp}$. This construct overlapps with 'Tc-six3 10down' for 426 bp. 'Tc-six3 10down' starts +4714 bp and ends $+9960 \mathrm{bp}$ downstream of the TLS of Tc-six3 with total size of 5246 bp (green line; Figure 11, Panel B).

All these amplified sequences were combined with the 'Tc-six 3 promoter element' and cloned in front of the reporter gene tGFP with adjacent SV40. Except the construct 'Tc-six3 5up', this was directly fused to tGFP with the adjacent SV40.

Taken together, four different fragments were amplified and cloned in front of tGFP and SV40. All of these have two TLS $18 \mathrm{bp}$ in front of the reporter gene tGFP. Additionally, the 'Tc-six3 promotor element', which was fused to all putative regulatory regions except to 'Tc-six3 5up', has a deletion of $9 \mathrm{bp}$. The assembled reporter constructs were used for transgenesis (chapter 2.6) and resulted in transgenic animals derived from two $\mathrm{G}_{0}$ animals with the cassette for 'Tc-six3 10up', from two $\mathrm{G}_{0}$ animals with the cassette for 'Tc-six3 $5 \mathrm{up}^{\prime}$, and from one $\mathrm{G}_{0}$ animal with the cassette for 'Tc-six3 5 down'. No animal could be successfully transformed with the cassette for 'Tc-six 310 down'.

\subsubsection{Analysis of transgenic animals carrying for the Tc-six 3 reporter line construct}

To test the function of the constructs in transgenic animals, in situ hybridizations with an antisense probe for tGFP were performed in $0-24 \mathrm{~h}$ and $24-48 \mathrm{~h}$ old embryos. Furthermore, the lines were scanned and analyzed for tGFP expression in the first larval instar (L1) brain. Generally, lines were denominated according to the number of the respective $G_{0}$, from which they derived and the number of the sibling line. For example, the third sibling crossing originated from $\mathrm{G}_{0} 7$ is further called line 7.3 .

\section{Transgenic lines carrying the construct 'Tc-six 310 up' $^{\prime}$}

For the cassette of 'Tc-six3 10up', two independent $\mathrm{G}_{0}$ could be generated, line 6 and line 7. From these, regarding sibling lines, which were analyzed according their reporter gene expression pattern, were generated.

In the case of line 6, only two sibling lines could be generated, lines 6.1 and 6.2. Both lines were analyzed for in situ hybridization and fluorescence signal of tGFP in the respective developmental stages. These lines showed an identical expression pattern, hence only embryos of line 6.2 are 
depicted in Figure 12. First, expression was detectable in embryos at late elongating stages (Figure 12, A). The construct drives tGFP expression distally in the appendages, except the mandibles (md; Figure 12). These distal expression domains remained present up to the oldest staged embryos analyzed here (Figure 12, C). In elongating embryos an expression domain was observed in the rim of the head lobes (Figure 12, $\mathrm{A}^{\prime}$ ). This domain decreased in size during ongoing development (Figure 12, B'). In fully elongated embryos, an additional expression domain arose within the labrum (Figure 12, B). After fusion of the labral segments, this expression pattern was still located in the anterior median part of the labrum (Figure 12, $\mathrm{C}^{\prime}$ ). The lateral expression domain within the rim of the head lobes was totally retracted to a small domain in the posterior part of the head, probably forming the larval eyes (Figure 12, C').
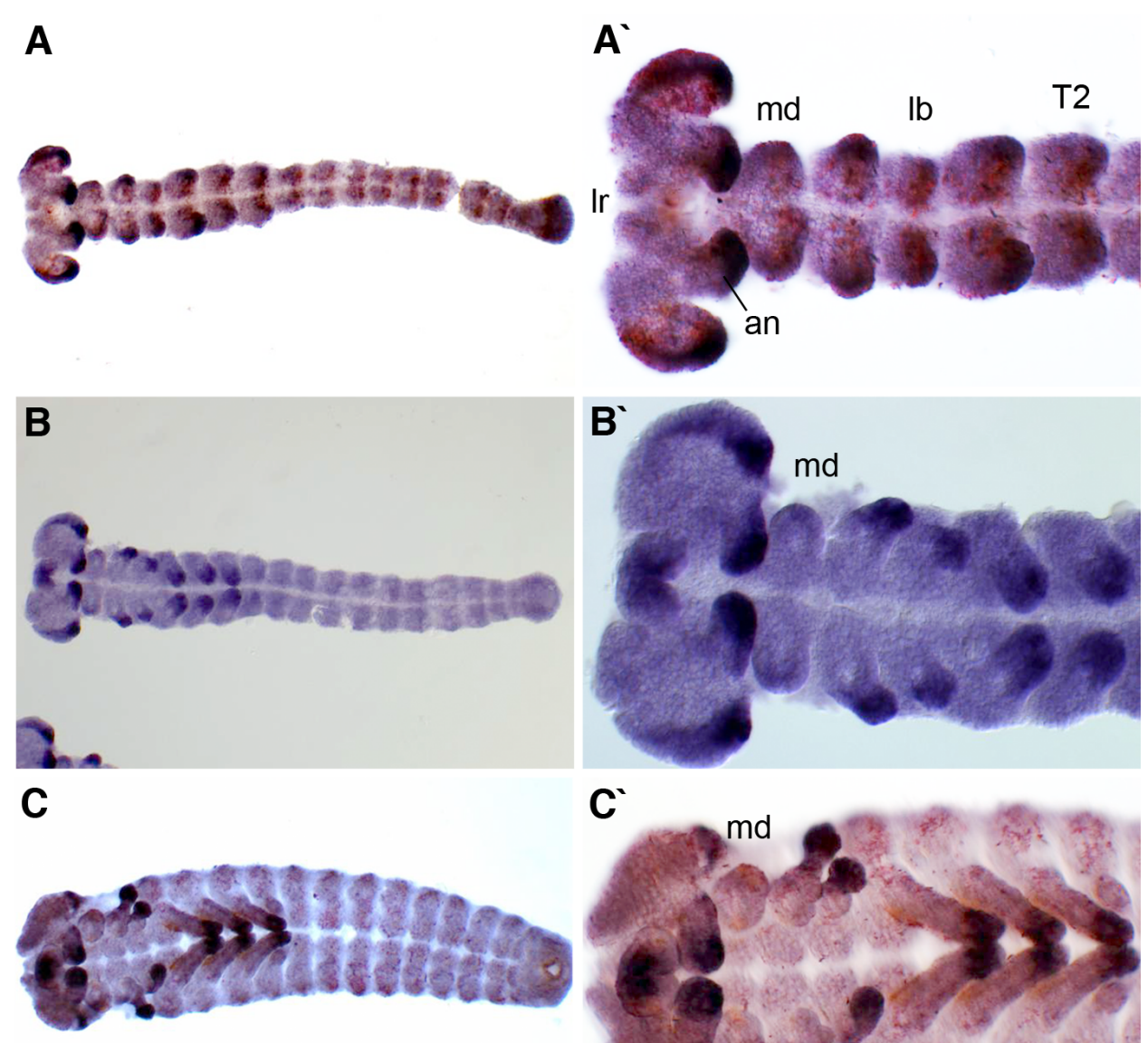

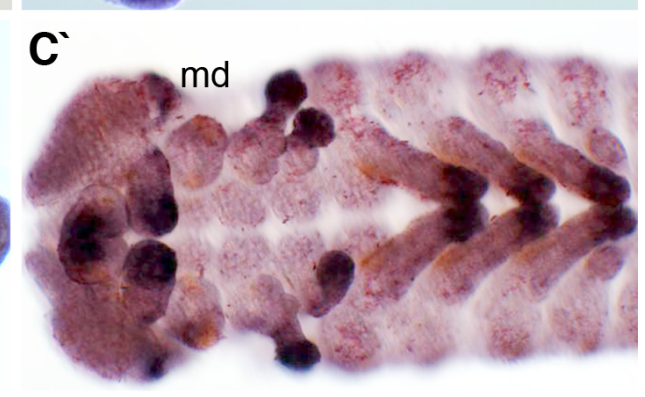

Figure 12: In situ hybridization with an antisense probe for tGFP in the transgenic line 6 containing the construct 'Tc-six 3 10up'.

(A) Elongating embryo, with expression in the rim of the head lobes and in the distal parts of budding appendages, except in the mandibles (md). (A') Close up of the upper part of the body. (B) Fully elongated embryo with tGFP-expression in the labrum and all distal parts of appendages, except the md. The lateral expression in the head fades. (B') Close up of the upper part of the body. (C) Fully retracted embryo with tGFP expression in the labrum and in the distal parts of appendages, except the md. ( $\left.\mathrm{C}^{\prime}\right)$ Close up to the upper part of the body, with the lateral head expression restricted to lateral posterior. (A, A', C, C') show double in situ hybridizations with an antisense probe against $T c$-wg depicted in red. lr: labrum; an: antenna; md: mandible; lb: labium; T2: thoracic segment 2. All embryos shown in total view are facing anterior to the left. All close ups to the head are facing anterior up. 
The expression pattern of the reporter line 'Tc-six3 10up' 6.1 and 6.2 reflects only to a small portion that of the endogenous gene Tc-six3. Thus, in the reporter lines derived from $\mathrm{G}_{0} 6$, the putative larval eyes domain (Figure 12, B and C) and the distal expression pattern in the labrum are the expression domains, which are overlapping with the endogenous expression pattern of the gene $T c$ six3. The further expression domains in the appendages of 'Tc-six3 10up' line 6.1 and 6.2 indicate an enhancer trap situation.

The reporter gene expression pattern distal in the appendages resembled the expression of $T c-d l l$ (Beermann et al., 2001). However, $T c-d l l$ is also expressed in the pleuropodia, which was not true for embryos of line 6 . Furthermore $T c-d l l$ has a lateral head domain only during early elongating stages (Beermann et al., 2001; Prpic et al., 2001), while such a domain was present in line 6 also in almost fully elongated embryos (Figure 12, B). The distal expression domain suggests the influence of an enhancer which drives expression in the distal appendages. The determination of the integration site of the construct 'Tc-six3 10up' could reveal an enhancer trap dependent expression pattern. I.e. an integration close to a leg gene like $T c$ - $d l l$ could be the reason for the observed expression. However, fluorescence in larval brains was not detected in line 6.1 nor line 6.2.

Sibling lines of $\mathrm{G}_{0} 7$ were also carrying the cassette for the 'Tc-six3 10up' construct. Three sisterlines were generated and analyzed for the expression pattern of tGFP.

The offspring of these lines was analyzed by in situ hybridization. All embryos showed the same expression pattern for the reporter gene tGFP, therefore only embryos of line 7.1 are depicted in Figure 13. The first expression pattern was detected in elongating embryos (Figure 13, A). Here, laterally in the head lobes, an elongated domain was strongly stained. In the median head region faint cellular expressions were detected (Figure 13, A'). During elongation, the lateral head domain as well as the median pattern were increased in intensity and spread out (black arrowhead, black arrow; Figure 13, B'). In the antennae and in the maxillae, faint expression domains arose de novo (open arrowhead, Figure 13, B` and Figure 13, B). In fully elongated embryos a faint distal expression pattern was detected in the appendages (Figure 13, C). This was located in the ectoderm and detectable distally in the labrum antennae, maxillae, labium and the legs, but not within the mandibles. Latter had two cellular expression domains on each side (open arrowhead, Figure 13, $\left.C^{\prime}\right)$. The lateral head domain was condensed to a lateral posterior expression in a region suggested to form the larval eye. Surrounding this domain, several spots were detected (black arrowhead, Figure 13, C'). At later stages, dotted domains were spreading all over the body (Figure 13, C and $\left.C^{\prime}\right)$. The distal superficial expression domains in the appendages could not be detected in older em- 

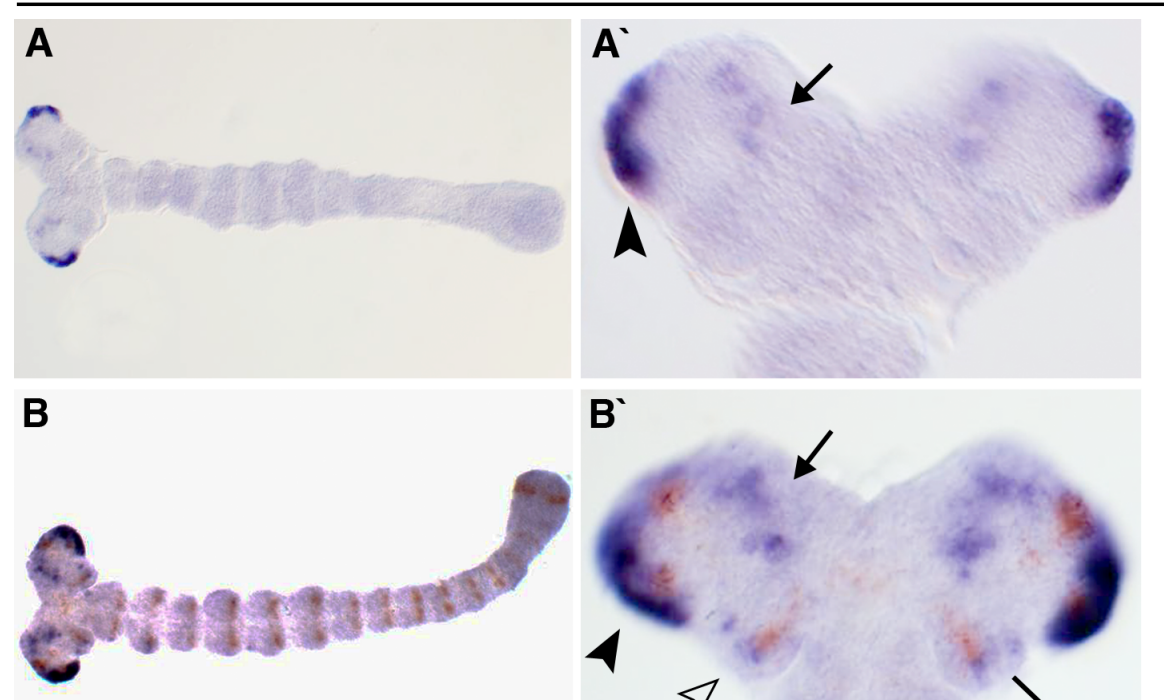

B
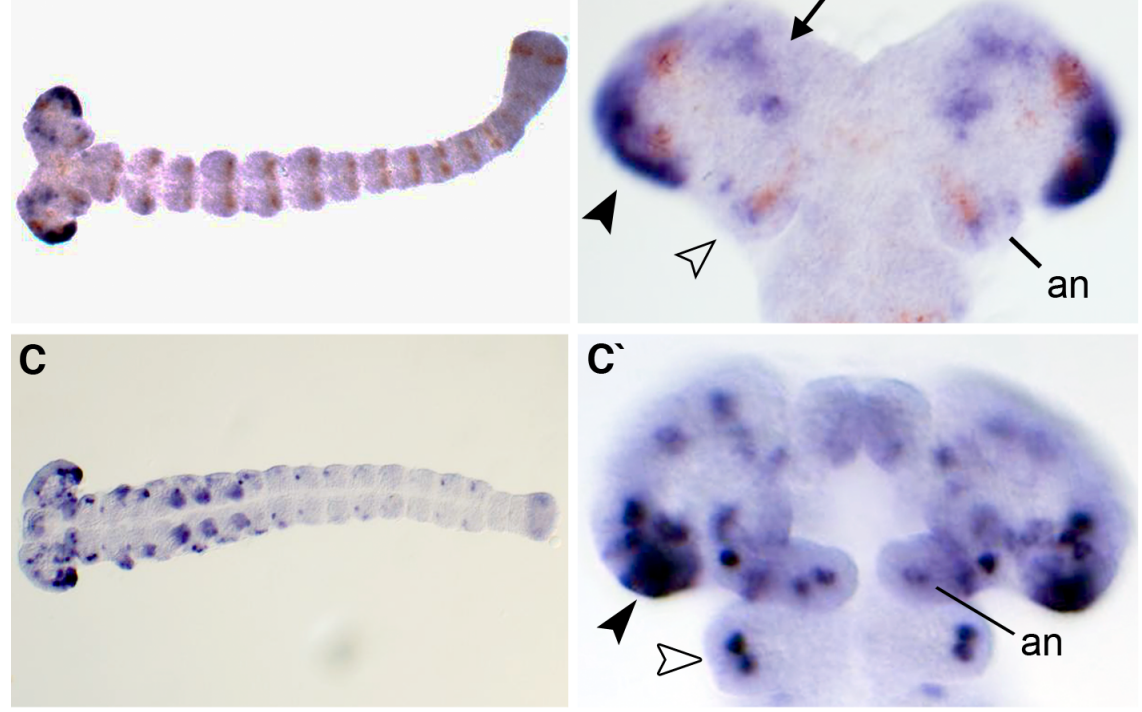

D
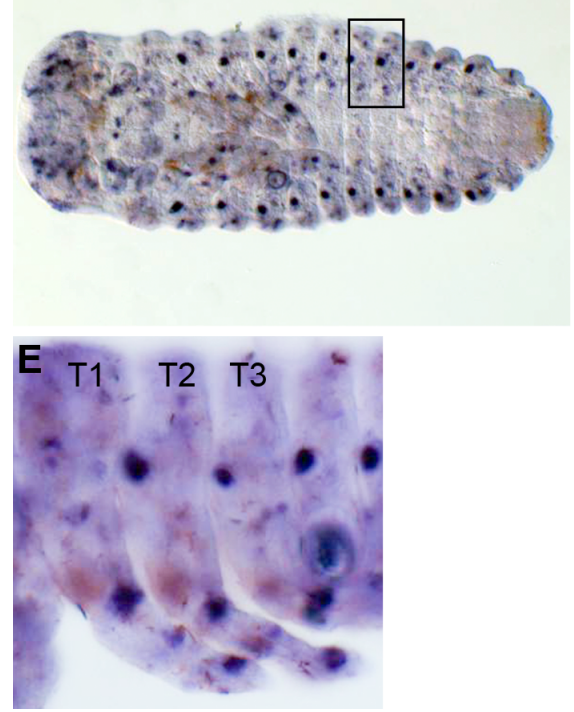

Figure 13: In situ hybridization with an antisense probe for tGFP in the transgenic line 7 containing the construct 'Tc-six3 10up'.

(A) Elongating embryo with the tGFP expression pattern laterally in the head. (A') Close up of the head, with the lateral expression domain (black arrowhead). The cellular median expression is pointed out by a black arrow. (B) Elongating embryo with exclusive expression in the head. (B') The lateral head expression pattern is shifted laterally posterior (black arrowhead). The medial domain is diffuse and connected to the lateral domain (black arrow). Weak expression is detected at the antenna basis (open arrowhead). (C) Fully elongated embryo, predominant expression in the upper part of the body, with the signal covering the distal tips of appendages, except mandibles. (C') Close up of the head with dotted expression domains within the head, especially in the lateral posterior region (black arrowhead). Segmental expression starting laterally in the mandibles (open arrowhead). (D) Approximately $48 \mathrm{~h}$ old embryo, dotted expression over the entire body. (D') Close up of the head, dotted domains scattered over the head. (D") Close up of abdominal segments, dotted expression in lateral and dorsal tissue with a prominent medial expression domain. (E) Same aged embryo as depicted in F. Lateral view of the legs with posterior expression domains up to the tip. All embryos shown in total view are facing anterior to the left. All close ups to the head are facing anterior up. 
bryos. Furthermore a complex spotty expression pattern covered the entire body (Figure 13, D). In the head, there were cellular expression domains (Figure 13, D'), which according to their location were possibly embedded in or part of the brain tissue. Interestingly, less expression was detectable ventrally in the embryo. The ventral abdomen was free of any expression domain, while a paired expression pattern was detected in the maxilla and first thoracic segment (Figure 13, D). However, the expression of tGFP was mainly located in lateral and dorsal tissues of the embryo. Here, many small spotty expressions could be detected, including one prominent, which in its location correlated to tracheal openings (Figure 13, D“). Such an expression domain was also visible in the third thoracic segment (T3), but this domain was smaller in comparison to the neighboring one. However, the first thoracic segment (T1) lacked such an expression domain. Additionally, dotted expression domains were detected posterior in the legs (Figure 13, E).

Thus, in comparison to the endogenous $T c$-six 3 expression pattern, only few expression domains of the line 7 are correlative or overlapping. During elongation, the faint expression pattern of tGFP median in the head (black arrow; Figure 13, $\mathrm{A}^{\prime}$ and $\mathrm{B}^{\prime}$ ) appears to be overlapping with the endogenous $T c$-six3 expression. Also in later stages, the cellular and spotty domains which are expressed in the labrum and in surroundings of the stomodeum, correlate to the endogenous Tc-six 3 expression (Figure 13, C' and D'). Interestingly, also the lines 7 appear to show a superficial and distal expression in the appendages, except the mandibles, only for a short period (Figure 13, C), like the line 6 (Figure 12). However, all the residual expression patterns of the line 7 do not show any domains correlating to the endogenous expression pattern of the gene Tc-six3. Rather, the pattern of line 7 is likely to have resulted from an enhancer trap.

The lines 7 were also analyzed for the fluorescence signal of tGFP in larval stages. Also here, the different sibling lines showed identical tGFP patterns. Therefore only line 7.3 will be described and is depicted in Figure 14. Single positive spots were detectable right below the cuticle and spread all over the head, which possibly indicated single cell bodies (open white arrowheads; Figure 14, B and C). Additional singular expression domains were detected around the oesophagus and in deeper sections tGFP positive cells could be detected laterally to the oesophagus (two anterior open white arrowheads; Figure 14, B). Also dorsally of the oesophagus, a group of single spots was present (open white square; Figure 14, C). In the anterior part of the head, a strong expression pattern projecting into the mandibles was detectable (white arrow; Figure 14, B).

Taken together, line 7 showed an embryonic expression pattern starting in the rim of the head. Also a distal expression pattern was detected in all appendages, except the mandibles. In later stages, the detected expression pattern became very complex with cellular as well as spotty domains spread 


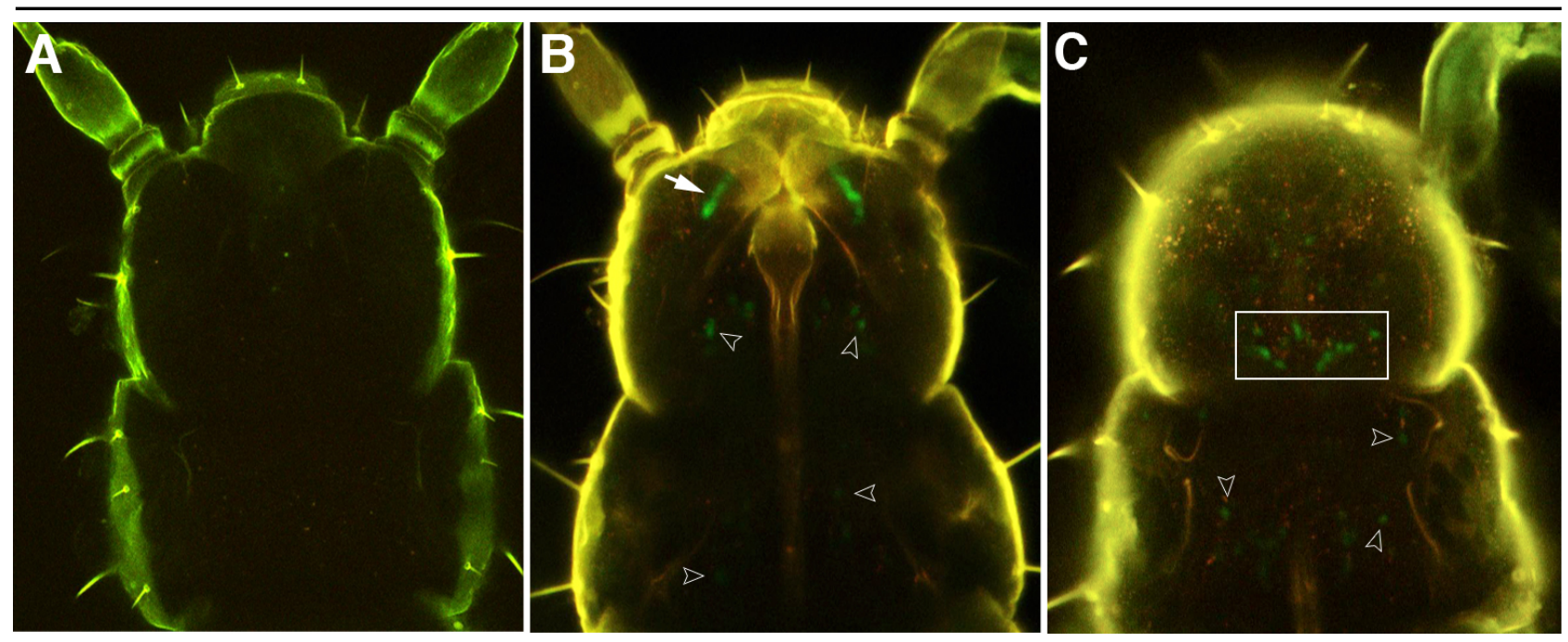

Figure 14: tGFP expression analysis in larvae of the transgenic line 7 carrying the construct 'Tc-six3 10up'.

(A) As a control, a $T c$-vermilion white larva in Green and Red was scanned. (B, C) Scans of line 7 transgenic for the construct 'Tc-six3 10up'. (B) Single tGFP expressing cells within the brain region are indicated by open white arrowheads, strong expression projecting into mandibles (white arrow) (C) Scan close to the dorsal cuticle. Group of single cells positive for tGFP dorsal to the oesophagus (white square), additional single positive cells are spread all over the brain (open white arrowheads).

all over the body. In the larval brain, singular cells expressing tGFP were detected throughout the brain and especially dorsally of the oesophagus. Furthermore a bilateral strong expression was detected projecting into the mandibles.

Two independent $\mathrm{G}_{0}$ were generated using the construct 'Tc-six3 10up'. In case of line 6, the expression of the reporter gene tGFP was only found in in situ hybridization analysis. In case of line 7, tGFP expression was detected by in situ hybridizations and confocal scans. Comparing the two lines, only the distal superficial expression pattern in the appendages, except in the mandibles, and the expression in the lateral rim of the head lobes were common. This suggests, that these expression patterns are caused by an enhancer element included in the 'Tc-six3 10up' sequence.

\section{Transgenic lines carrying the construct `Tc-six3 5up’}

For the 'Tc-six3 5up' reporter construct (chapter 3.1.3.2), three lines from different $\mathrm{G}_{0}$ with the serial numbers 8,9 , and 10 were generated. The offspring of the sibling lines originated by $\mathrm{G}_{0} 8$ were negative for in situ hybridization and fluorescence analysis.

In case of $\mathrm{G}_{0} 9$, only one line could be established. This line 9.1, showed a weak tGFP expression pattern in the labrum starting in elongating embryos (Figure 15, A and $\mathrm{A}^{`}$ ). After retraction, two expression domains could be detected in the labrum (Figure 15, B') and furthermore a weak expression in the pleuropodia. In later stages, additional expression domains arose at the basis of the 
labrum (open arrowhead; Figure 15, C'), while the other two expression domains in the labrum still remained (black arrowhead, Figure 15, C'). Furthermore, tGFP mRNA expression was detected in the proctodeum and in the pleuropodia (Figure 15, C). However, no fluorescence signal of tGFP was detected in larval offspring of line 9.1.

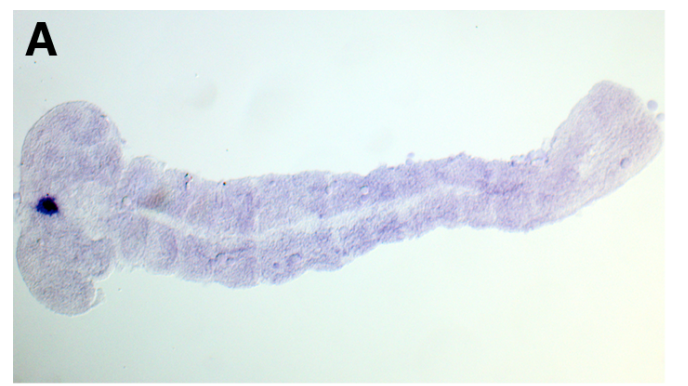

B

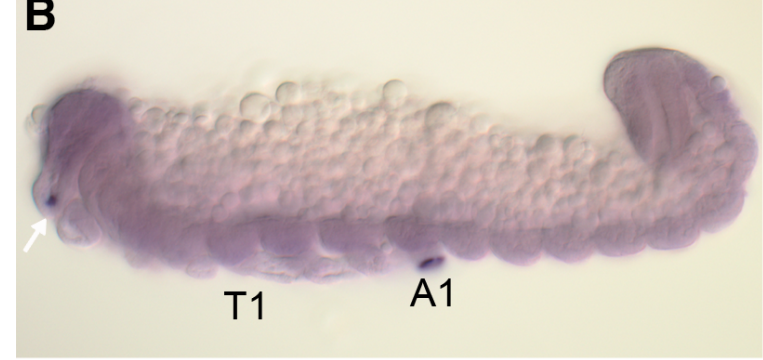

C

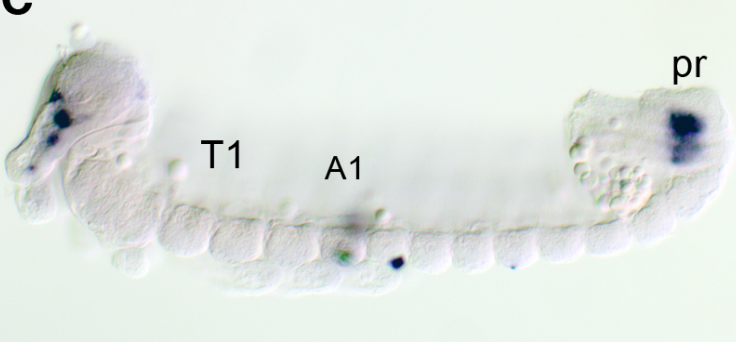

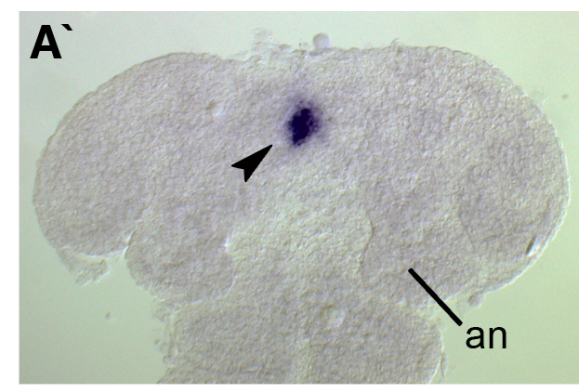
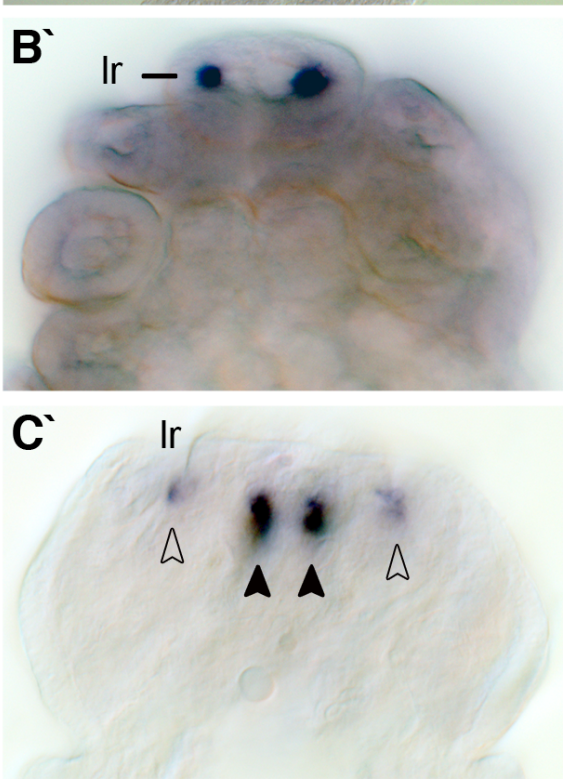

Figure 15: In situ hybridization with an antisense probe for tGFP in the transgenic line 9 containing the construct 'Tc-six 3 5 up'.

(A) Ventral view of an elongating embryo with dotted expression in the anterior median head. (A') Close up of the head, a black arrow points out the expression domain. (B) Lateral view on a fully retracted embryo. (B`) Ventral view of the labrum (lr) with two tGFP expression domains. (C) Lateral view of a approximately $30-35 \mathrm{~h}$ old embryo with expression domains within the labrum and proctodeum (pr). (C') Close up of the ventral view of the labrum with two stronger expression domains (black arrowhead) and weak expression domains (open arrowhead). All embryos shown in total view are facing anterior to the left. All close ups to the head are facing anterior up. Indicated ages correlate to the development at $32^{\circ} \mathrm{C}$.

Two sibling lines were derived from $\mathrm{G}_{0} 10$. Lines 10.2 and 10.3 showed identical expression patterns in in situ hybridization for tGFP. The expression pattern started in posterior pit stages, when the blastoderm was already differentiated and the germ rudiment started invagination. Here, the expression of tGFP was detectable in the ventral median of the germ rudiment (Figure 16, A) along the mesoderm. This expression pattern remained present in elongating embryos (Figure 16, B and $\mathrm{C}$ ). This ventral domain fainted during elongation and a new expression domain arose in the developing labrum (Figure 16, C and C'). Eventually, the mesodermal expression pattern could not 

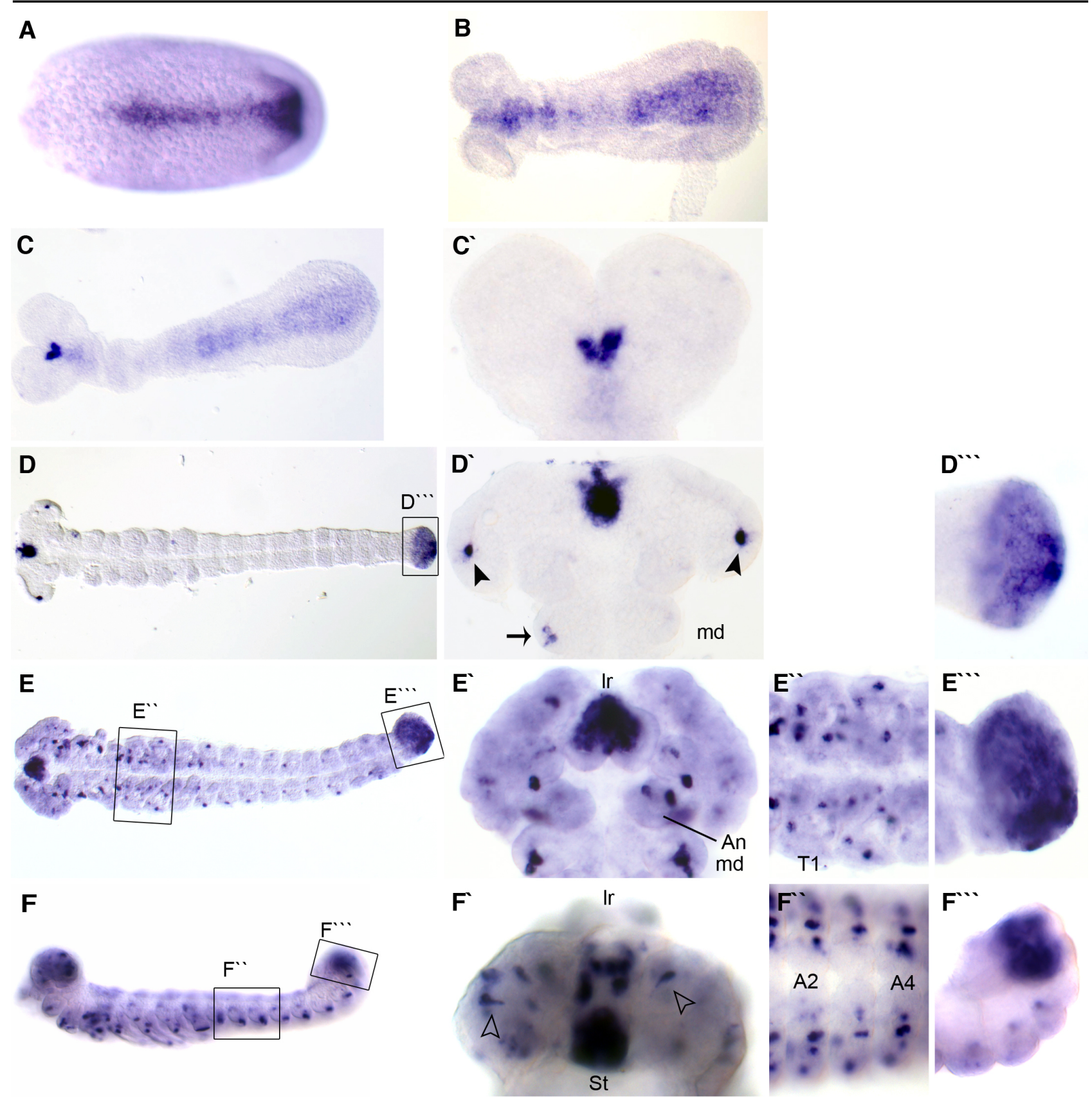

Figure 16: In situ hybridization with an antisense probe for tGFP in the transgenic line 10 containing the construct ' $T c$ six3 5 up'.

In all embryos depicted in total view, anterior is facing to the left. (A-E) Embryos depicted ventrally, (F) depicted leterally. (C'-F') Close up of embryonic heads oriented anterior upward. (A) Germ rudiment with a mesodermal expression pattern. (B) Early elongating embryo with a mesodermal expression pattern. (C) Fainting mesodermal expression pattern, arising of strong stomodeal expression. (C') Close up of the head, with strong expression. (D) Almost fully elongated embryo, strong expression surrounding the labrum. (D') Close up of the head, with strong expression surrounding the labrum basis. Point-shaped expression laterally in the head lobes (black arrowhead) and in the mandible (blak arrow) segment. (D ') Close up of the growth zone, with strong expression covering the posterior tip. (E) Fully elongated embryo before retraction, with a dotted segmental expression pattern and strong expression in the anterior and posterior tissue. (E') Close up of the head, with strong expression in the labrum and dotted expression scattered over the head. (E') Close up of the thorax T1 with a dotted expression pattern. (E'”) Strong expression pattern covering the posterior tissue. (F) Lateral view of a retracted embryo, with a strong dotted expression pattern in the head and all segments. (F') Strong expression at the interior stomodeal opening and in cells stretching out (open arrowheads). 
$\left(F^{\prime \prime}\right)$ Close up of the abdominal segments (A2-A4) with 4 dotted cell clusters lateral on each side. (F”) lateral view of stomodeum.

be detected in fully elongated embryos (Figure 16, D). The labral domain grew in size and in the lateral rims of the head lobes two point-shaped domains arose (black arrowhead, Figure 16, D'). The proctodeum was also positive for tGFP expression (Figure 16, D“'). Only in the mandibles a faint spotty expression pattern was present (black arrow; Figure 16, D), while a complex expression pattern arose in later stages (Figure 16, E and F). In embryos close to retraction, a strong expression was present centrally in the labrum (Figure 16, E') and scattered domains were arising in the head and spreading posterior. Within the thorax, each segment had a complex pattern (Figure 16, E“'). Strong expression of tGFP mRNA was detected at the surface layer of the proctodeum (Figure 16, $\left.\mathrm{E}^{\prime \prime}\right)$. In older embryos, the segmental dotted expression pattern was present in all segments (Figure 16, F). The labral domain was retracted to the interior part of the stomodeum, and in the labrum two point-shaped domains were present distally (out of focus). Cells positive for tGFP expression were bottle shaped and expanded interior (open arrowhead, Figure 16, F'). Within the appendages, a group of dotted domains was detected in the distal part and only single domains were detected proximally. In the proctodeum, tGFP mRNA was expressed strongly (Figure 16, F'”).

Contrary to the strong expression pattern detected by in situ hybridization, no signal could be detected by confocal scans in the respective L1 larvae.

Taken together, lines 9 and 10, which were derived from two $\mathrm{G}_{0}$, showed common tGFP expression patterns in the labrum and in the proctodeum. While the labral expression possibly reflects endogenous Tc-six3 activity, the activity in the proctodeum is not seen in anti Tc-six3 in situ hybridization experiments.

\section{Transgenic lines carrying the construct 'Tc-six3 5down'}

Two $\mathrm{G}_{0}$ animals with the reporter construct 'Tc-six3 5down', numbered 2 and 3, could be created by transgenesis. From each $\mathrm{G}_{0}$, three sibling lines were generated by crossings with Tc-vermillion. However, the sibling lines originated from the parental $\mathrm{G}_{0} 2$ were negative for in situ hybridization and for fluorescence analysis of the reporter gene.

Interestingly, the same was found for the lines 3.1 and 3.2. Only the third crossing (3.3) was positive for in situ hybridization analysis for tGFP. Here, a pair rule gene like expression pattern was observed during elongation, starting in early germ bands close to the serosa and amnion fusion (Figure 17, A). This expression pattern remained present until the closure of the serosa window 

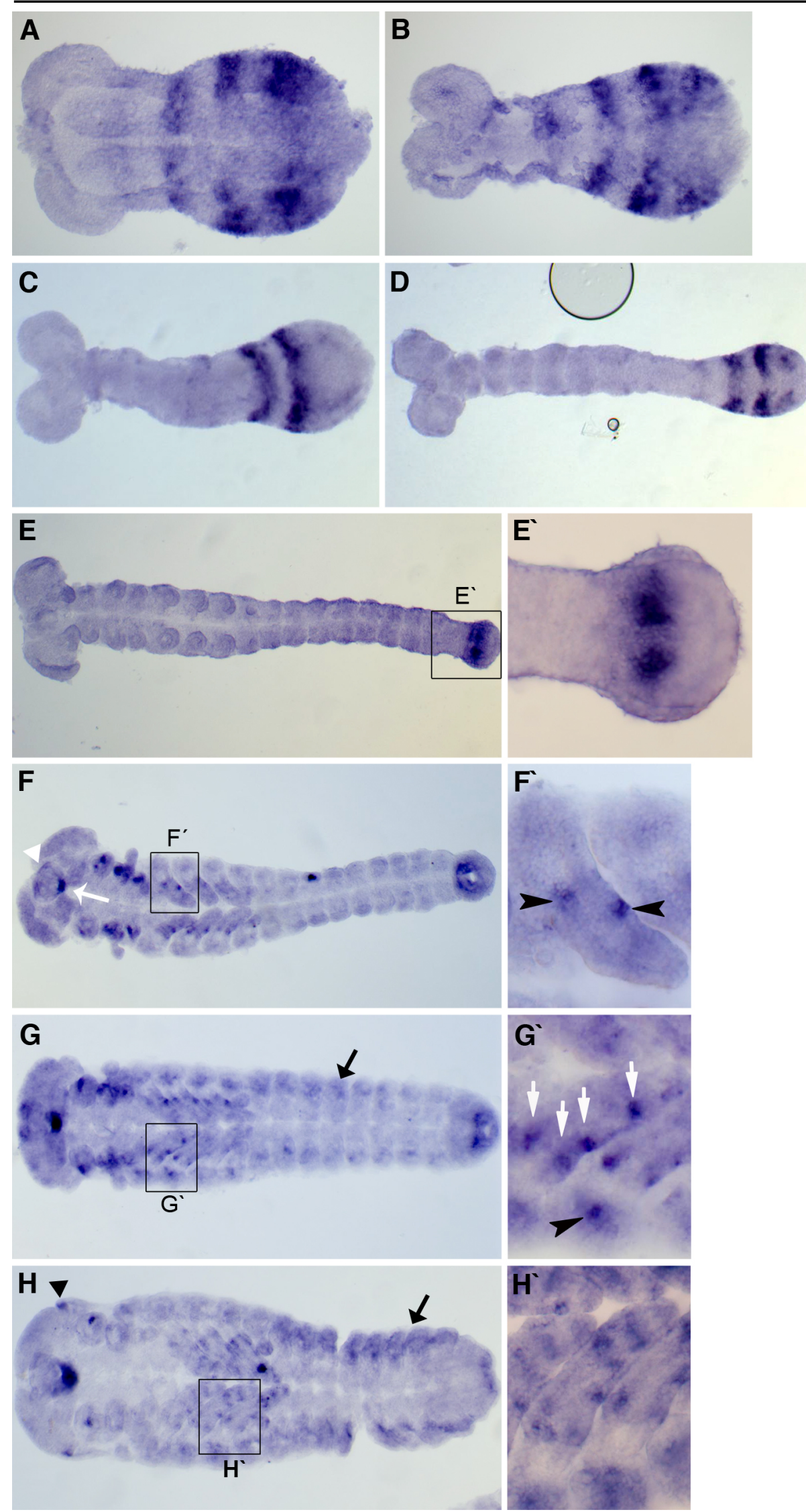

Figure 17: In situ hybridization with an antisense probe for tGFP in the transgenic line 3 containing the construct ' $T c$ six3 5down'.

(A) An approximately $10 \mathrm{~h}$ old embryo with a pair rule gene like expression pattern. (B) An approximately $12 \mathrm{~h}$ old embryo with a pair rule gene like expression pattern. (C, D) Approximately $14 \mathrm{~h}$ and $16 \mathrm{~h}$ old embryos with pair rule like expression patterns. (E) An approximately $18 \mathrm{~h}$ old embryo with an expression domain in the putative proctodeum anlage. (E') Close up of the putative proctodeum anlage. (F) An approximately $24 \mathrm{~h}$ old embryo with an expression pattern anterior in the labrum (white arrowhead) and labrum basis (white arrow), as well as in the gnathal buds. Dotted expression pattern in the legs and roundish expression domain in the proctodeum. (F') Close up of the leg. (G) 30-35h old embryo with a similar expression pattern compared to the embryo depicted in $\mathrm{F}$; further domains arise segmentally in dorsal tissue (black arrow). (G') Dotted expression pattern in the legs (white arrows) and in dorsal tissue of the thorax segment T2 (black arrowhead). (H) An approximately $45-50 \mathrm{~h}$ old embryo

with a similar expression pattern as the embryo depicted in $\mathrm{G}$ and in addition point-shaped expression laterally in the head (black arrowhead), stronger expression in dorsal tissue. ( $\mathrm{H}^{\prime}$ ) Close up of legs with complex expression pattern. All embryos shown in total view with the anterior facing to the left, all close up images with the anterior facing to the left. Indicated ages correlate to the development at $32^{\circ} \mathrm{C}$. 
(Figure 17, B). During elongation, a striped expression pattern was detectable only in the posterior segmentanlagen close to the growth zone (Figure 17, C and D). This pattern could not be detected in fully elongated embryos. In these stages, the tGFP expression was observed only in the proctodeum (Figure 17, E and E'). A posterior expression pattern surrounding the proctodeum opening remained detectable throughout all developmental stages (Figure 17, F, G, and H). De novo domains arose in retracting embryos. Expression in the gnathal appendages (Figure 17, F) as well as a spotty expression pattern, which arose in the legs were detected (Figure 17, F'). Furthermore, a small expression domain appeared around the stomodeum (white arrow; Figure 17, F) and increased in size during development (Figure 17, G and H). In dorsal tissue a superficial expression arose and spread from anterior to posterior (black arrow; Figure 17, G). In the legs, the expression domains were more dotted (Figure 17, G') and were more complex in older embryos (Figure 17, H'). The expression in dorsal tissue shifted posterior and the gnathal expression pattern could no longer be detected, except in the mandibles (Figure 17, H).

Considering the fact that only one line was generated, it is hard to distinguish wethter the expression pattern is influenced by enhancer elements of the construct 'Tc-six3 5down' or by local enhancer elements close to the insertion side. Nevertheless, this line does not show the expression pattern of Tc-six 3 .

\subsection{3. $T c-r x$ reporter lines}

Parallel to the $T c$-six3 reporter lines, transgenic lines for the gene $T c-r x$, expressing the reporter gene DsRedExpress (DsRedEx) in the same pattern, were to be generated. To this end, the endogenous promoter was analyzed and the 5'UTR was identified by two independent RACE-PCR experiments (chapter 2.2.). The regulatory regions were amplified by PCR of gDNA of the SB strain. The respective sequences were analyzed for gene predictions and repetitive sequences. Finally, the generated transgenic lines were analyzed regarding their expression pattern of the reporter gene during embryonic stages as well as in the larval brain.

\subsubsection{Generation of the $T c-r x$ reporter lines}

The analysis of the 5'UTR of Tc-rx by RACE-PCR revealed a total length of $19 \mathrm{bp}$. Tc-rx has a typical insect TSS sequence (reviewed in Butler and Kadonaga, 2002; highlighted blue, Figure 18, Panel A). Further studies identified a downstream promoter element at position +28 relative to the TSS (DPE; highlighted blue, Figure 18, Panel A). This element was located within the first ORF of Tc-rx. To include the DPE within the endogenous promoter element, a primer, which also replaced 
the endogenous translation start site ATG with ATA, was designed (black line, Figure 18, Panel A). Hence, an element of $625 \mathrm{bp}$ was cloned as endogenous promoter and was fused with all Tc-rx reporter constructs (purple line under 5up construct; Figure 18, Panel B) except 'Tc-rx 5up', where this region was already included (purple line; Figure 18, Panel B).

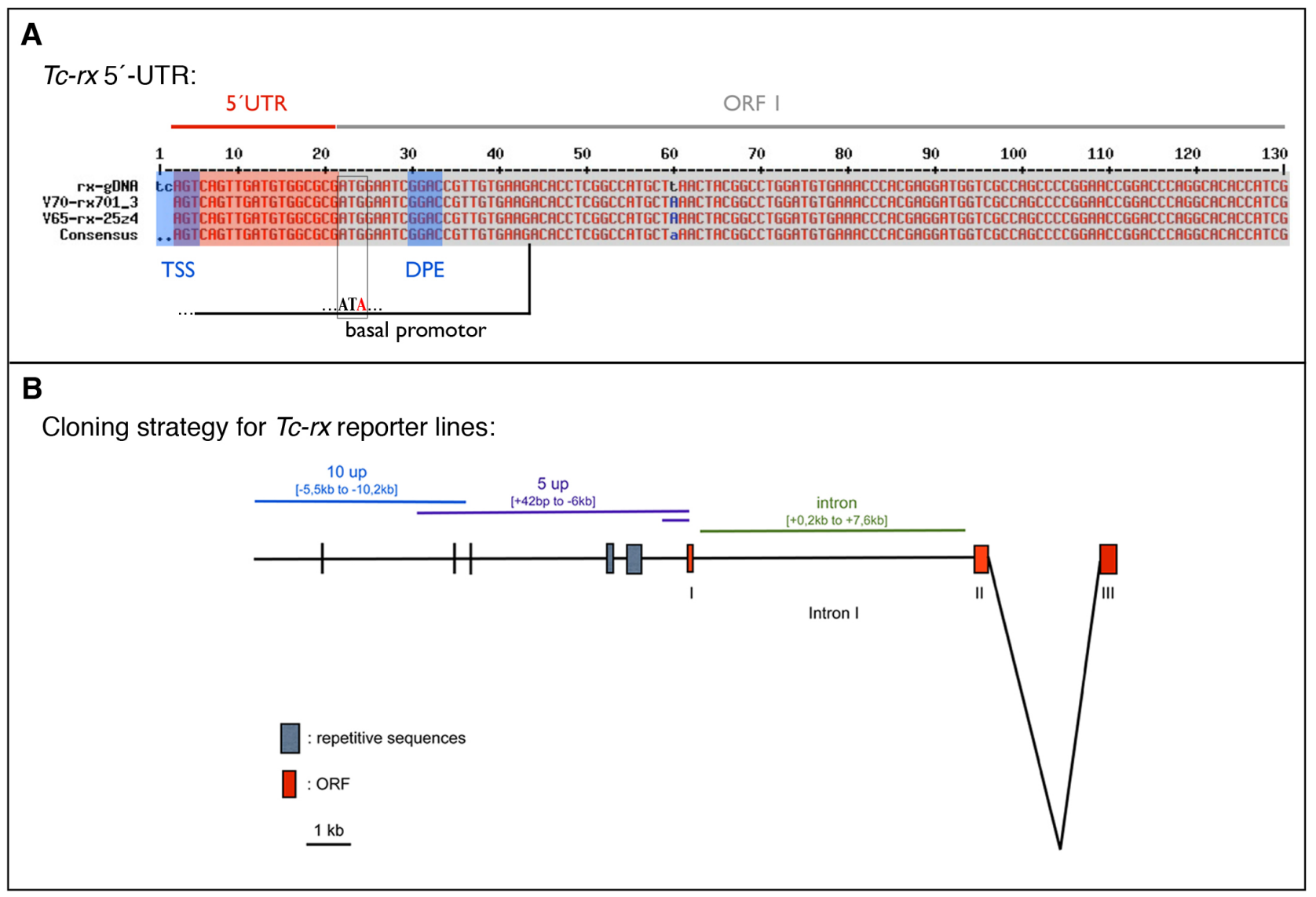

Figure 18: The 5'UTR and the cloning strategy for the transgenic reporter lines of $T c-r x$.

(A) The 5 UTR of Tc-rx is 19 bp long. It has a transcription start site (TSS) with the typical consensus sequence. A downstream promoter element (DPE, highlighted blue) was found +28 bp of the TSS, within the ORF1 (highlighted gray). The DPE was included in the basal promoter (black line) and the native translation start site was disrupted by replacing $\mathrm{G}$ with A. (B) A basal promoter of $625 \mathrm{bp}$ was cloned including the entire 5 UTR and the DPE (depicted as a shortened line under the 5 up construct). The sequences $10 \mathrm{up}$ and 5 up overlap by $577 \mathrm{bp}$. The regulatory regions were combined with the endogenous basal promoter driving DsRedExpress, except for the construct 'Tc-rx 5up', which already included the basal promoter. The 'Tc-rx Intron' was cloned in front of the basal promoter. Transgenic lines for various $T c$ - $r x$ reporter gene constructs were generated: lines with the construct 'Tc-rx 5up' (highlighted in purple) and lines with the construct 'Tc-rx Intron' were generated and tested positively in in situ and in vivo experiments regarding DsRedEx expression. No transgenic line was generated with the construct 'Tc-rx 10up' (highlighted in blue). (A) 5 'UTR is highlighted in red, Exon regions are highlighted in gray, and TSS elements highlighted in blue; (B) Repetitive sequence regions are depicted in gray boxes; ORFs are depicted in red boxes; predicted gleans are depicted in pink boxes. 
The sequence for the construct 'Tc-rx 10up' started -10248 bp and ended -5491 bp upstream of the translation start site of Tc-rx (TLS; blue line; Figure 18, Panel B). It had a total size of $4757 \mathrm{bp}$ and overlapped for $577 \mathrm{bp}$ with the adjacent 'Tc-rx $5 \mathrm{up}^{\prime}$ 'sequence. The sequence for the construct 'Tc-rx $5 u^{\prime}$ started $-6068 \mathrm{bp}$ upstream of the TLS of Tc-rx and ended +22 within the ORF 1 of Tc-rx (purple line; Figure 18, Panel B). The construct 'Tc-rx Intron' was located within the first intron of $T c-r x$ and was 7598 bp long. Nearly the complete first intron was included in the construct, starting at $+213 \mathrm{bp}$ and ending $+7611 \mathrm{bp}$ downstream of the TLS of Tc-rx (green line; Figure 18, Panel B), $174 \mathrm{bp}$ upstream of ORF II.

Control sequencing revealed no disorders. Transgenic offspring from one $\mathrm{G}_{0}$ animal for the cassette for 'Tc-rx 5up::DsRedEx' and one $\mathrm{G}_{0}$ for the cassette for 'Tc-rx Intron::DsRedEx' could be generated. However, no animal was successfully transformed with the cassette for 'Tc-rx 10up::DsRedEx'.

\subsubsection{Analysis of transgenic animals carrying the $T c-r x$ reporter line constructs}

For analysis of the functionality of the constructs in transgenic animals, in situ hybridization with an antisense probe for DsRedEx was performed. Therefore, egg lays were staged 0-24h, 24-48h, and $48-72 \mathrm{~h}$ at $32^{\circ} \mathrm{C}$. Additionally, the first larval instar brains (L1) of individuals of the transgenic lines were scanned for the fluorophor DsRedEx.

\section{Transgenic lines carrying the construct 'Tc-rx 5up'}

In the offspring originating from the $G_{0} 1$, two animals were positive for the transfection marker carrying the cassette 'Tc-rx 5up::DsRedEx' and were established into sibling lines. Interestingly, only line 1.2 was positive for the reporter gene DsRedExpress, indicating independent transposition events.

The expression of DsRedEx mRNA started late in development. The first expression was detected in embryos with approximately 30-40h. The expression pattern was exclusively present in the head (Figure 19, A). Three paired expression domains were detected. One median domain was present close to the surface in the dorsal part of the head (open black arrowhead; Figure 19, A“). This domain expanded interior ventrally. At deeper levels, two lateral domains, which were located along the anterior-posterior axis, were observed (white arrowheads, Figure 19, A'). In later stages, these domains were shifted posterior in parallel to the lateral axis (white arrowheads; Figure 19, B') and lateral to the median domain, where they showed a cellular expression (open black arrowhead; Figure 19, B'). A de novo expression domain arose at the ventral anterior segment boundary of the 

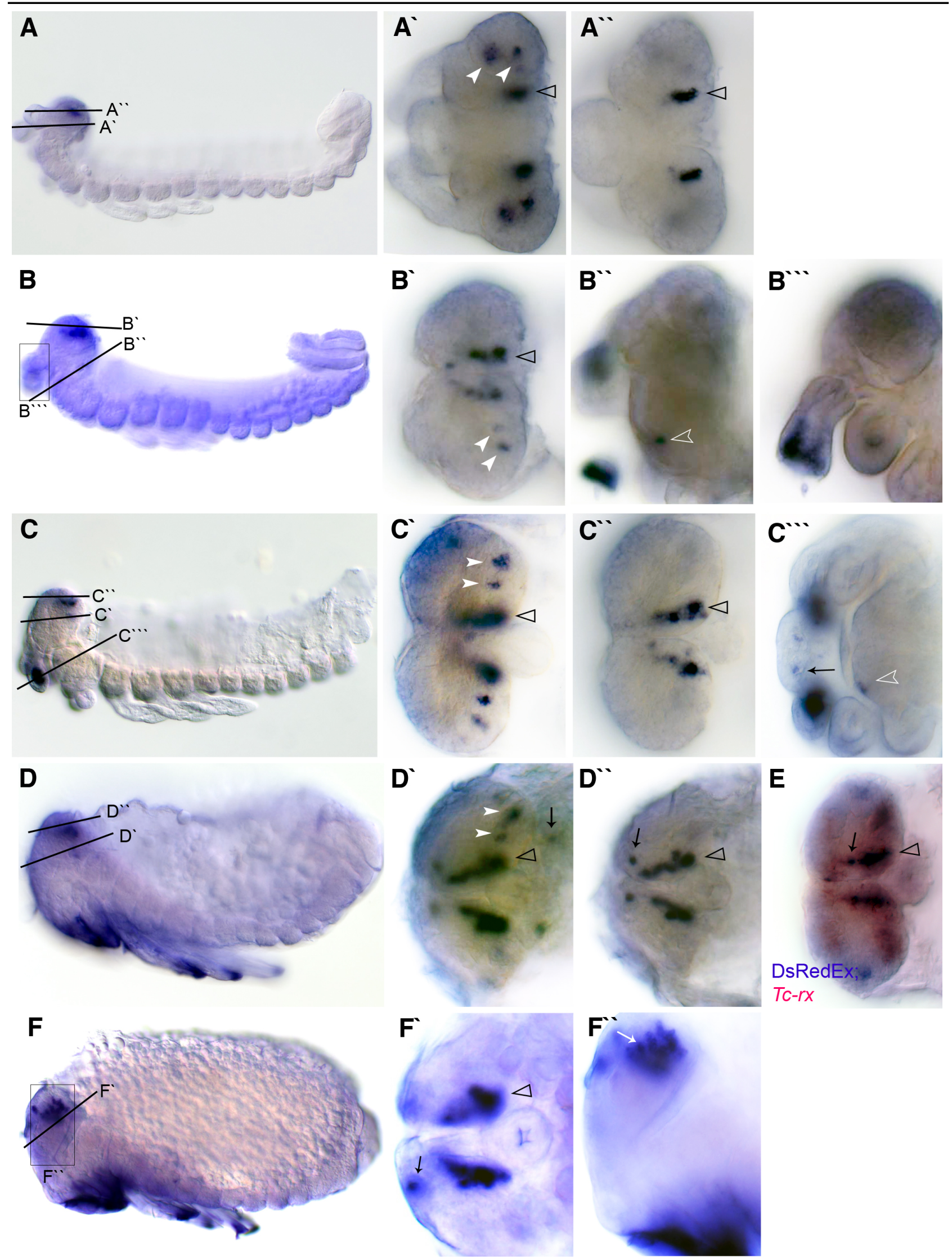

Figure 19: In situ hybridization with an antisense probe for DsRedEx in the transgenic line 1 containing the construct 'Tc-rx 5up'.

(A-E) Lateral views. Horizontal cross sections are indicated by lines, lateral cross sections are indicated by squares. Lateral expression domains are indicated by white arrowheads. The median expression domain is indicated by an open black arrowhead. Arrows and arrowheads are only shown in one hemisphere. All indicated ages correlate to the 
development at $32^{\circ} \mathrm{C}$. (A) The expression pattern was first detectable in $30-40 \mathrm{~h}$ old embryos. (A') Horizontal cross section of the lateral expression domains. (A") Horizontal cross section at the surface with a median expression domain. (B) 40-50h old embryo. (B') Horizontal cross section with unchanged median and changed lateral expression domains. (B") Symmetric expression domain at the anterior tip of the mandibular segment (open white arrowhead). $\left(B^{\prime \prime \prime)}\right.$ Lateral cross section with an expression domain at the distal tip of the antenna. (C) 50-60h old embryo. (C') Horizontal cross section with lateral expression domains. (C") Horizontal cross section of the median expression domain at the surface. $\left(\mathrm{C}^{\cdots}\right)$ ) Ventral view with expression pattern at the tip of the mandibular segment (open white arrowhead). The distal tip of the antennal expression domain is indicated (out of focus). Two domains arise de novo in the labrum (black arrow). (D) Embryo older than 60h. (D') Close up of the lateral expression domains, the mandibular expression domain indicated by a black arrow. (D") Two domains arise de novo (black arrow) anterior of the median expression domain. (E) Double in situ hybridization with antisense probe for Tc-rx (red) and DsRedEx (blue). Tc-rx is expressed in the epidermis above the median expression domain. (F) Embryo older than 65h. (F') Median expression domain with anterior expression domain (black arrowhead). ( $\left.\mathrm{F}^{\prime \prime}\right)$ Lateral cross section with the median expression domain and elongated cells (white arrow).

mandibular segment (open white arrowhead, Figure 19, B" '). Furthermore, strong expression of the reporter gene was found in the distal part of the antennae (Figure 19, B "'). The expression pattern did not change much in older stages (Figure 19, C). Here, the paired domains laterally in the head were detectable (white arrowheads; Figure 19, C'), the dotted median domains expanded over several z-sections (open black arrowhead; Figure 19, C' '), the distal expression in the antennae got stronger (Figure 19, C and C') and the dotted domain remained ventral anterior at the segment boundary of the mandibular segment (open white arrowhead, Figure 19, C'”). Two cellular expression domains in the labrum were additionally found (black arrow, Figure 19, C'”). The expression of DsRedEx mRNA remained restricted to the head and gnathal segments, even in embryos older than 60h (Figure 19, D). A small point-shaped expression arose anterior to the median expression pattern, which in the meanwhile had resolved into several cellular expression domains (open black arrowhead; Figure 19, D“). The stages that started with secretion of the cuticle developed background staining (Figure 19, D and F). Hence, in embryos older than 65h, the mandibular and labral expression was not detectable anymore, due to the background. The median expression domain was still located to brain tissue (open black arrowhead; Figure 19, F') and consisted of cellular expression domains including anterior spots (black arrow; Figure 19, F'). Cells positive for DsRedEx expression within the brain tissue showed an elongated shape (white arrowhead; Figure 19, F“). DsRedEx mRNA was still detectable in embryos close to hatch.

Double in situ hybridizations with antisense probes for $T c-r x$ and the reporter gene DsRedEx were performed in embryos of the transgenic line 'Tc-rx 5up:.DsRedEx'. Even though the obtained results were preliminary, the median expression domain (open black arrowhead; Figure 19; E) was 
located right below the superficial expression pattern of $T c-r x$ (Figure 19, E). Also the anterior small roundish domain in front of the median domain overlapped with $T c-r x$ expression (black arrow; Figure 19, E). Due to the limitations of light microscopy in deeper layers, a colocalization analysis based on confocal scans needs to be performed. Here, fluorescently labeled antibodies could be used to stain the proteins of $T c-R x$ and DsRedEx. Therby an analysis of putative colocalization of both proteins in the cells of the median domain in the 'Tc-rx 5up' line would be possible.

A fluorescent signal was detected in the L1 brains of individuals of the 'Tc-rx 5up' line. Posterior dorsal groups of cells sent projections towards anterior (white square, Figure 20, A). This projection crossed the interhemispheric brain fissure, thereby forming a chiasma (white arrowhead; Figure 20, A). Singular expression domains are detectable throughout the head, in most cases suggested to be neural tissue. Paired groups of cells positive for DsRedEx were detectable under the cuticle surface in the labrum (white arrow; Figure 20, C). A second, bigger and paired group of cells was located anterior of the cervix (white arrow; Figure 20, A and B) and an additional group of cells was found posterior (white arrow; Figure 20, $A^{`}$ and $B^{`}$ ). A further signal was detectable within the brain, with no cellular, but thin projections (open white arrowhead; Figure 20, A and A`). A cellular expression pattern with thin elongations was observed within the antennae (Figure 20, A "). For a better allocation of the DsRedEx signaling caused by the 'Tc-rx 5up' reporter construct, crossings with other brain imaging lines were performed. For this, the line 6xP3::eCFP and the 'Mushroom Body' $\left(\mathrm{MB}^{\prime}\right)$ line, that originated from the GEKU-screen (chapter 3.1.4), were chosen.

The posterior group of cells forming the chiasma (white square; Figure 20, A) was embedded by cortex glial net like tissue (white square; Figure 20, B) and located median posterior to the endpoint of the vertical lobe of the Mushroom Body (white square; Figure 20, C). The position of the chiasma lay dorsally within the brain, above the median lobe (white arrowhead; Figure 20, C). This region, as well as the group of cells anterior to the cervix, was also covered by a net of cells which were glial cells (white arrow; Figure 20, B). This was also true for those cells embedded in the posterior glial tissue (white arrow; Figure 20, B'), surrounding the Kenyon cells of the Mushroom Body (Figure 20, $C^{\prime}$ ). The thin projections within the brain described above were surrounding the Central Body neuropile. A stronger signal was detected anterior of the Central Body, expanding laterally in the protocerebrum (open white arrow; Figure 20, B' and C'), which could represent parts of the lateral accessory lobes. A DsRedEx signal could only be detected surrounding the Kenyon cells, but was not detected within the Mushroom Body (Figure 20, C'). Finally, the antennal cellular DsRedEx positive cells surrounded the axon bundles marked by $6 x P 3:: \mathrm{eCFP}$ (Figure 20, B“'). 

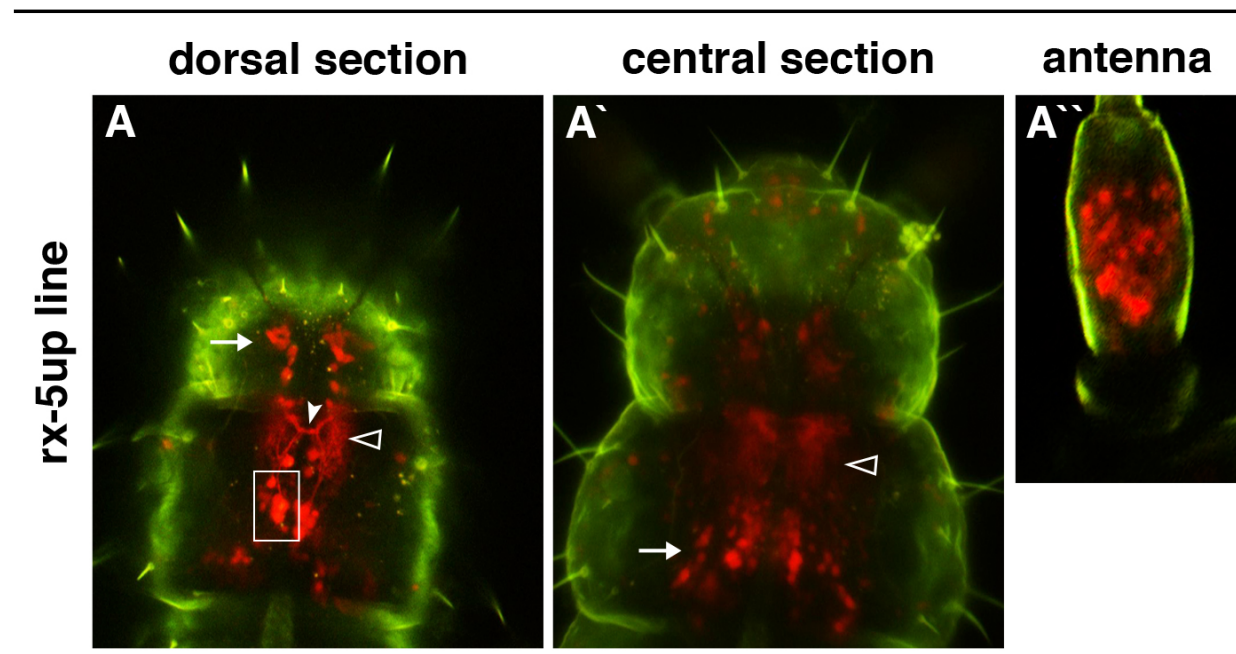

Figure 20: Analysis of reporter gene expression in the brain of a larva transgenic for 'Tc-rx 5up'.

(A-A ") The DsRedEx signal in the transgenic line 'Tc-rx 5up'. (A) Dorsal sections of the brain; groups of cells projecting towards anterior (white square), forming a chiasma (white arrow-
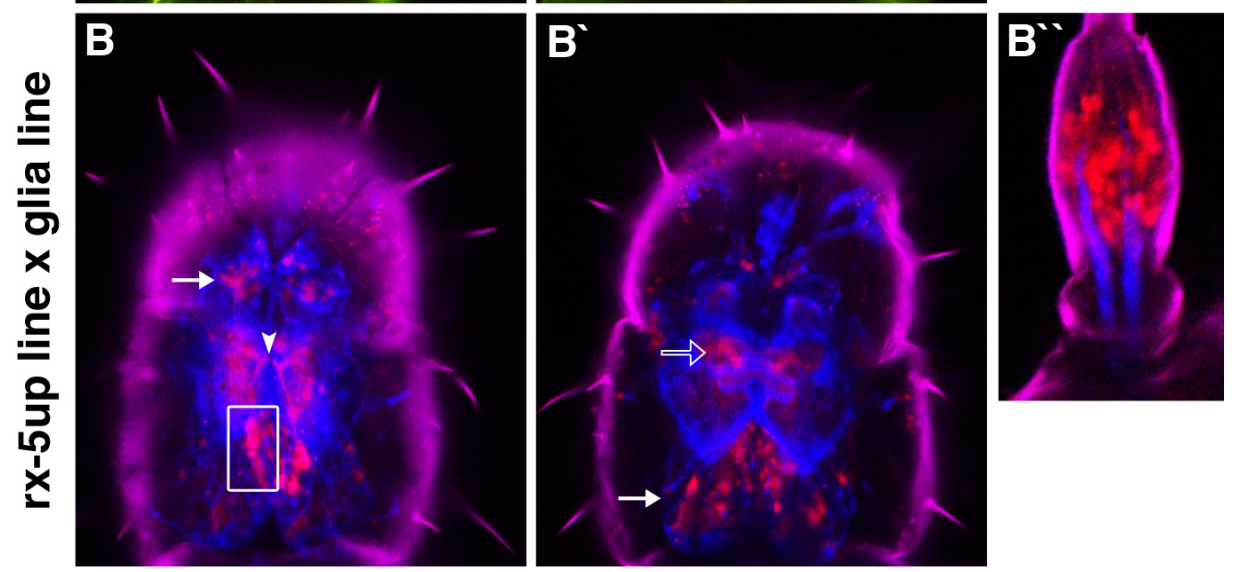
head); a net of thin projections is indicated by an open white arrowhead. (A') Deeper section of the brain; groups of cells posterior in the brain (white arrow); net of thin projections indicated by open white arrowhead. (A ') Zoom into the antenna. (B-B ') Crossing of the lines 'Tc-rx 5up::DsRedEx' shown in red and $6 \times \mathrm{P} 3:$ : eCFP shown in blue. (B) Dorsal sections of the brain; groups of cells forming the chiasma surrounded by glia (white square); chiasma (white arrowhead); anterior group of cells (white arrow).

(B') Deeper section of the brain; posterior group of cells

surrounded by glia (white arrow); putative lateral accessory lobes (open white arrow) (B") Zoom into the antenna, blue axon bundle within cell bodies positive for DsRedEx. (C-C') Crossing of the lines 'Tc-rx 5up::DsRedEx' shown in red and the 'MB' line depicted in blue. (C) Dorsal section of the brain; the vertical lobe, parallel blue structure to white line; labral group of cells (white arrow); chiasma (white arrowhead) (C') Deeper section of the brain; putative lateral accessory lobe (open white arrow). Anterior is facing up.

Taken together, DsRedEx was first detected in embryos of the line 'Tc-rx 5up::DsRedEx' at an age of approximately $30-40 \mathrm{~h}$ by in situ hybridization eperiments. The exclusive expression pattern in the head and the gnathal segments is suggested to mark specific groups of cells throughout development and even so in L1 brains. The fluorescence signal of DsRedEx revealed a complex but 
distinct expression pattern within the larval brain. The posterior group forming the chiasma could possibly be part of the pars intercerebralis and/or the pars lateralis. However, these cells probably derived from the median domain during embryonic stages, which were overlapped by $T c-r x$ expression. This specific pattern suggests the presence of a specific enhancer element within the reporter construct 'Tc-rx 5up'. However, due to the lack of comparative lines, influences of the insertion locus on the expression pattern of DsRedEx cannot be excluded. Nevertheless, the line 'Tc-rx 5up::DsRedEx' represents a potentially valuable imaging line for further studies regarding the observed lineage positive for DsRedEx as well as additional aspects, e.g. axogenesis.

\section{Transgenic lines carrying the construct 'Tc-rx Intron’}

For the construct 'Tc-rx Intron', it was possible to identify marked offspring from one $\mathrm{G}_{0} 11$. From this $\mathrm{G}_{0}, 3$ sibling lines were generated 11.1, 11.2 and 11.3. These showed DsRedEx mRNA expression in the embryo and a fluorescent signal in larval stages.

All sibling lines showed a range of diverse but in principle similar expression patterns. Therefore only line 11.1 and the most representative embryos are described here. The expression of DsRedEx mRNA was first detected in old embryos. In elongated embryos, a group of cells at the basis of the labrum was positive for DsRedEx mRNA (Figure 21, A`). A weak dotted expression started to be detectable laterally in the gnathal appendages (Figure 21, A ") as well as in the legs (Figure 21, $\left.\mathrm{A}^{\prime \prime}\right)$. In additional embryos, single domains positive for DsRedEx mRNA were found in abdominal segments or even in the proctodeum (not shown).

In embryos that had finished the retraction, more domains, which in some cases spread all over the embryo, were detected. The labral expression pattern elongated laterally anterior (black arrow; Figure 21, B'). At the same level laterally in the head, two point-shaped, in most cases paired domains were often detected (black arrowhead; Figure 21, B'). Laterally in the gnathal appendages, single spots were detectable (Figure 21, B“'), while similar spotty expression domains were localized anterior in the legs (Figure 21, B“'). Single domains were spread all over the body in an irregular fashion (not shown). This was also observed for the expression pattern in the head. After retraction, the labral domains fused proximally (Figure 21, $C^{\prime}$ ). The expression pattern in the appendages became more superficial but remained in the anterior part (Figure 21, C" and C'”). In old embryos (Figure 21, D), dotted expression domains were spread all over the body. Those in the appendages were most prominent and at the labrum basis a group of smaller expression domains formed a belt (black arrowhead; Figure 21, D'). Paired expression domains were found in the antennae (Figure 21, D') and strong expression was also detected in the distal parts of the gnathal 

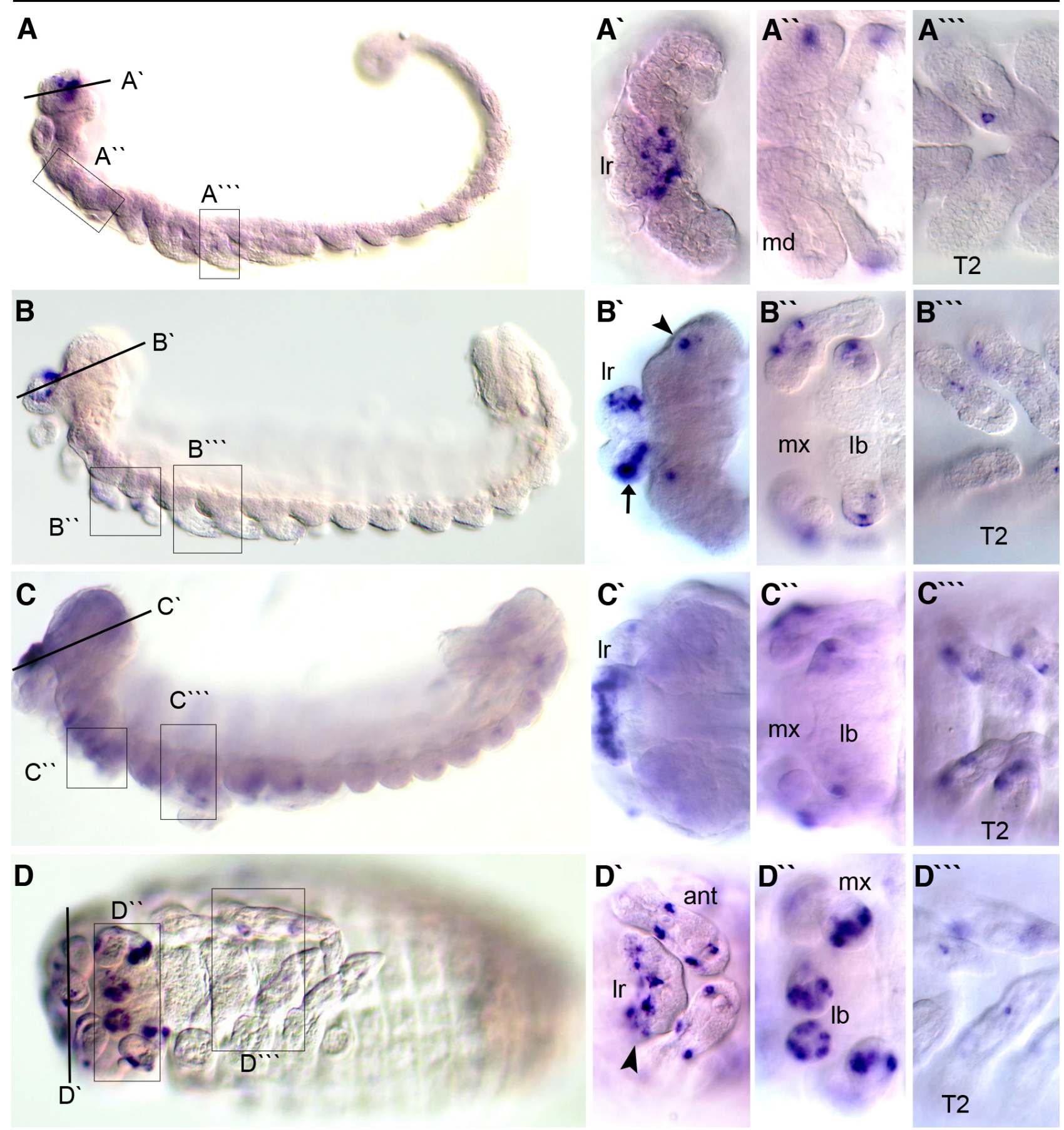

Figure 21: In situ hybridization with an antisense probe for DsRedEx in the transgenic line 11 containing the construct 'Tc-rx Intron'.

(A-C) Lateral views of embryos of different age, except (D) which shows a ventral view. (A) An approximately $24 \mathrm{~h}$ old embryo, where the first expression pattern was detectable in the head. (A') Horizontal cross section: expression pattern in the basis of the labrum (lr). (A“) lateral expression domains within the gnathal segments mandibel (md) and maxilla (mx). (A $\left.{ }^{\prime \prime}\right)$ The dotted expression domain anterior in the leg bud T2. (B) Embryo 25-30h old. (B') Horizontal cross section; lateral expression in the $1 \mathrm{r}$ (black arrow) and two point-shaped expression domains within the head (black arrowhead). (B“) Expression domain anterior in the gnathal segments $m x$ and labium (lb). (B“') Small expression domain in the leg. (C) Embryo approximately 40-45h old. (C') Horizontal cross section; fused labral expression pattern. $\left(C^{\prime \prime}\right)$ Anterior expression pattern in the $\mathrm{mx}$ and $\mathrm{lb}$. (C'”) Split dotted expression pattern anterior in the legs. (D) Embryo approximately 55-60h old. (D') Frontal view of the $1 \mathrm{r}$ and antennae (ant), dotted expression patterns in the $1 \mathrm{r}$ 
basis (black arrowhead) and ant. (D“) Expression patterns distal in the tips of the mx and lb. (D“') Weak expression in the legs. In all pictures anterior are facing to the left. All indicated ages correlate to the development at $32^{\circ} \mathrm{C}$.

appendages (Figure 21, D“). Interestingly, the expression pattern in the legs reflected the pattern detected in the antennae (Figure 21, D“'). In some cases, a superficial expression was observed in the dorsal tissue. This was located one-sided along the anterior-posterior axis in the dorsal tissue in a different region in each embryo (not shown).

The expression patterns described here represent those which all lines derived from $\mathrm{G}_{0} 11$ have in common. In comparison to the expression pattern of Tc-rx, none of the described expression patterns in the lines 11.1 - 11.3 showed a correlation, except the labral one. However, despite the difference of the embryonal expression patterns of the lines 11.1 - 11.3, the fluorescent signals within L1 heads were surprisingly similar. Therefore only one individual of line 11.1 will be described here. Cellular expression was scattered within the head capsule and probably within the brain (Figure 22, B). Some of these domains were connected with each other. Especially in the median region of the protocerebrum, very weak superficial domains were detected (open white arrowheads; Figur 22, B and C). Slightly anterior to the cervix, a group of DsRedEx positive cells formed a globular structure through their projections (white arrow, Figure 22, C). Cellular expression, possibly displaying the soma of sensory neurons, was found in the labrum (Figure 22; $\left.\mathrm{B}^{\prime}\right)$ and in the antennae (Figure 22; C'). From these cellular expressions, projections, which were detected in the tip of the antennae and in the head, arose (Figure 22, $\mathrm{C}^{\prime}$ ). The latter terminated in a network of DsRedEx positive tissue (white arrowhead; Figure 22, B). The most prominent expression domain was found within the labrum (white square; Figure 22, B). Putative sensory neurons were projecting to the distal tip of the labrum (Figure 22, B'). The projections into the head ended near those of the antennae, but not in the same structure (not shown). For further analysis, detailed localization by crossings with the glial line $6 x \mathrm{P} 3$ or with the 'MB' line are suggested.

Taken together, the sibling lines 11.1 - 11.3 showed a similar fluorescent pattern in the larval head, which only partially correlated with the DsRedEx mRNA expression found within the embryos. The variability of the embryonic expression pattern suggests that either more constructs were integrated in the genome or each sibling displays independent integrations. Both cases should be tested by inverse PCR. Further studies by crossings with the glial marker line and the 'MB' line could be performed, and by this the expression domains in the larval head could possibly be related to neuromeres or neuropiles. However, only one $\mathrm{G}_{0}$ could be generated and therefore it is hard to 
elucidate whether the described patterns are influenced by enhancer elements surrounding the integration locus or present in the 'Tc-rx Intron' construct.
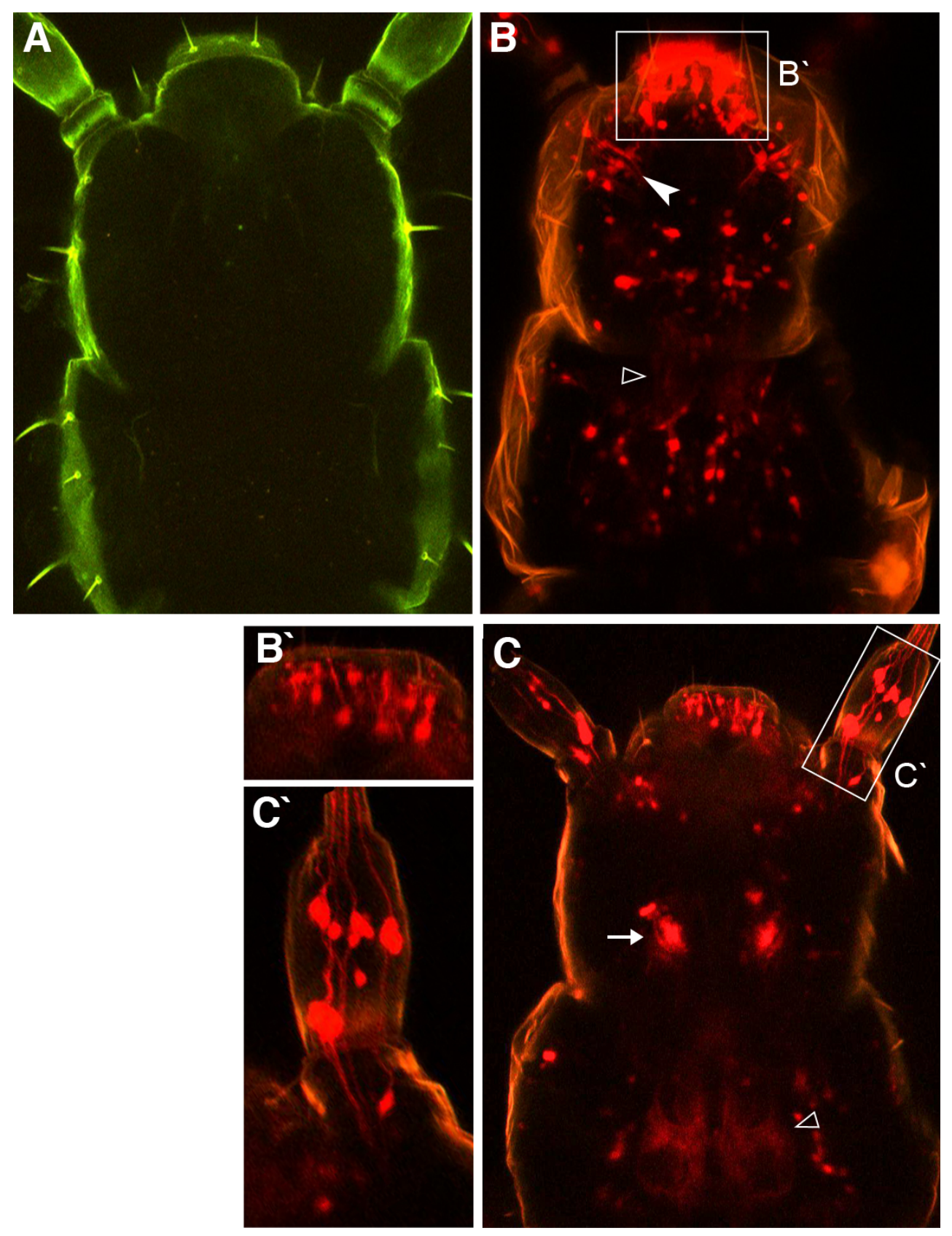

antenna, single positive cells with projections. Anterior facing up.
Figure 22: Analysis of reporter gene expression in the brain of a transgenic larva for 'Tc-rx Intron'.

(A) As a control, a $T C$ vermilion larva was scanned in green and red. (B C) Scans of a 'Tc-rx Intron' positive larva. (B) Dorsal section of the head; scattered expression domains within the brain region and strong antennal projections (white arrowhead). Superficial domain, probably formed by thin projections (open arrowhead) (B') Close up of the labrum with single cell bodies and projections. (C) Deeper section within the head; with cells form a globular domain (white arrow). Superficial domain, which is putatively formed by thin projections (open white arrowhead). (C') Close up of the 


\subsubsection{Verification of transgenic reporter lines by PCR and schematic summary of the characterization of the transgenic animals}

At least 29 putative lines were analyzed regarding their reporter gene expression patterns in embryos by in situ hybridizations as well as in larvae by confocal scanning for fluorescent signals in the larval head. However, only 13 lines showed expression of the respective reporter gene in embryonic stages. From those, only 8 lines showed a fluorescent signal in the larval head (compare Figure 24). Thus, PCR experiments on genomic DNA of transgenic animals were performed (Figure 23) in order to clarify, whether the additional G1 with black eyes but without expression pattern in the embryo or in the larva, were false positive or whether no signal could be detected due to low expression of the reporter gene caused by the integration site.

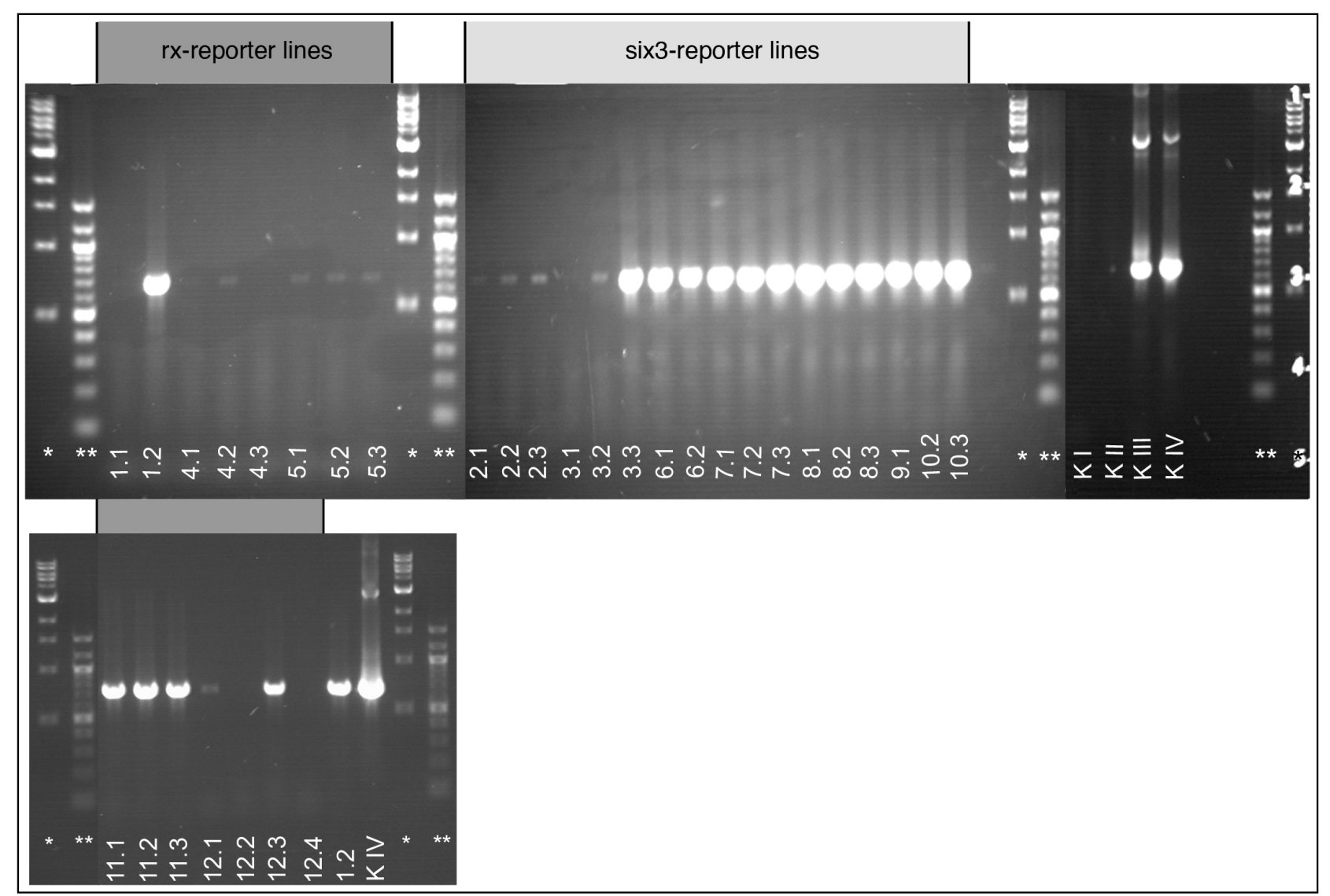

Figure 23: PCR analysis of $T c-r x$ and $T c-s i x 3$ reporter lines by screening gDNA for reporter gene insertion.

Lines 1.1 and 1.2 carried the construct 'Tc-rx 5up'; lines $4.1-4.3$ and $5.1-5.3$ should carry the construct 'Tc-rx 10up'; lines $11.1-11.3$ and $12.1-12.4$ should carry the construct 'Tc-rx Intron'. The reporter gene DsRedEx is not included in the gDNA of lines $4.1-4.3,5.1-5.3$, and 12.1, 12.2, 12.4. Lines $2.1-2.3$ and $3.1-3.3$ carried the construct 'Tc-six3 5down'; lines 6.1 - 6.2 and 7.1 - 7.3 carried the construct 'Tc-six3-10up'; lines 8.1 - 8.3, 9.1, 10.2 and 10.3 carried the construct 'Tc-six3 5up'. The reporter gene tGFP is not included in the gDNA of lines 2.1 - 2.3, 3.1, and 3.2. KI, KII were negative controls: gDNA of Tc-ver strain with primer for DsRedEx and tGFP, respectively. KIII was a positive control: PCRII-tGFP plasmid with tGFP primer. KIV was a positive control: PCRII-DsRedEx plasmid with DsRedEx primer. *:1kb ladder; **: 100bp ladder 
Sibling lines of $\mathrm{G}_{0} 2,3,6,7,8,9$, and 10 carrying the Tc-six 3 reporter constructs were analyzed by PCR for tGFP insertion in their genome. The animals positive for tGFP showed a distinct band of approximately $650 \mathrm{bp}$. Hence, the lines derived from $\mathrm{G}_{0} 6,7,8,9$, and 10 were positively tested by PCR (Figure 23). The residuals showed only a weak band, which was also detectable for gDNA of SB-wildtype animals and of Tc-vermilion animals, depending on the amount of gDNA used in the experiment (not shown). Therefore, these weak bands are regarded as negative results, which support the negative results in in situ hybridization and in confocal scanning experiments. Therefore, the offspring lines for $\mathrm{G}_{0} 2$ and the lines 3.1 and 3.2 were negative for an insertion of the reporter constructs (Figure 23). In these lines no expression of the reporter gene could be identified by in situ hybridizations and confocal scanning experiments.

Interestingly, within lines derived from $\mathrm{G}_{0} 3$, only line 3.3 was positive for in situ hybridization experiments. There are several possibilities why lines 3.1 and 3.2 were positive for the transfection marker Tc-vermilion (black eyes), but negative for the reporter construct. First, a contamination with animals of the SB-line could have occurred, since crossings were performed in pupal stages and young pupae have no pigmented eyes. Second, a possible contamination with animals of $T c$ pearl strain could have occurred, which per se also have white eyes, but after crossings to animals of the Tc-vermilion line produce black-eyed offspring.

The PCR analysis was positive for the reporter gene tGFP in the genome of the offspring lines of $\mathrm{G}_{0}$ 8. Contrary to this, expression analyses by in situ hybridization and confocal scanning were negative, indicating that the 'Tc-six3 5up' reporter construct possibly inserted into a silencing genomic region.

For $T c-r x$ reporter constructs, the sibling lines of the $\mathrm{G}_{0}$ animals $1,4,5,11$, and 12 were analyzed. Those animals positive for DsRedEx showed a distinct and strong band of approximately $650 \mathrm{bp}$. Hence offspring lines of $\mathrm{G}_{0} 1,11$, and some of 12 were positive.

Interestingly, line 1.1 was also positive for insertion analysis (not shown), but not for in situ hybridization or confocal scans. This could be explained by integration in a silenced or silencing locus in the genome, repressing any detectable expression. Additionally, to test the hypothesis of separate insertion loci for line 1.1 and 1.2 in detail, an inverse PCR could be performed.

Individuals of the line 12.3 were positive for DsRedExpress, but were not analyzed further, because confocal scans revealed a contamination with the 'Brainy' line (chapter 3.1.4).

The following scheme summarizes the results of the described characterization experiments for the transgenic animals carrying the $T c-s i x 3$ and $T c-r x$ reporter gene constructs. 


\section{Table 1: six3-reporter lines}

\begin{tabular}{|c|c|c|c|c|c|c|c|c|c|c|c|c|c|c|c|c|c|}
\hline \multirow[b]{2}{*}{\begin{tabular}{|l} 
six 3 -line \\
\end{tabular}} & \multicolumn{5}{|c|}{ six3-10up } & \multicolumn{6}{|c|}{ six3-5up } & \multicolumn{6}{|c|}{ six $3-5 d o$} \\
\hline & 6.1 & 6.2 & 7.1 & 7.2 & 7.3 & 8.1 & 8.2 & 8.3 & 9.1 & 10.2 & 10.3 & 2. 1 & 2.2 & 2.3 & 3.1 & 3.2 & 3.3 \\
\hline $\begin{array}{l}\text { PCR } \\
\text { analysis }\end{array}$ & $\checkmark$ & $\checkmark$ & $\checkmark$ & $\checkmark$ & $\checkmark$ & $\checkmark$ & $\checkmark$ & $\checkmark$ & $\checkmark$ & $\checkmark$ & $\checkmark$ & $x$ & $x$ & $x$ & $x$ & $x$ & $\checkmark$ \\
\hline $\begin{array}{l}\text { in situ } \\
\text { stainings }\end{array}$ & $\checkmark$ & $\checkmark$ & $\checkmark$ & $\checkmark$ & $\checkmark$ & $x$ & $x$ & $x$ & $\checkmark$ & $\checkmark$ & $\checkmark$ & $x$ & $x$ & $x$ & $x$ & $x$ & $\checkmark$ \\
\hline $\begin{array}{l}\text { promotor } \\
\text { deletions }\end{array}$ & yes & yes & yes & yes & yes & no & no & no & no & no & no & yes & yes & yes & yes & yes & yes \\
\hline $\begin{array}{l}\text { confocal } \\
\text { scans }\end{array}$ & $x$ & $x$ & $\checkmark$ & $\checkmark$ & $\checkmark$ & $x$ & $x$ & $x$ & $x$ & $x$ & $x$ & $x$ & $x$ & $x$ & $x$ & $x$ & $x$ \\
\hline
\end{tabular}

Table 2: rx-reporter lines

\begin{tabular}{|c|c|c|c|c|c|c|c|c|c|c|c|c|}
\hline \multirow[b]{2}{*}{$r x-$ line } & \multicolumn{6}{|c|}{ rx-10up } & \multicolumn{2}{|c|}{ rx-5up } & \multicolumn{4}{|c|}{ rx-Int } \\
\hline & 4.1 & 4.2 & 4.3 & 5.1 & 5.2 & 5.3 & 1.1 & 1.2 & 11.1 & $\overline{11.2}$ & 11.3 & 12 \\
\hline \begin{tabular}{|l|} 
PCR \\
analysis
\end{tabular} & $x$ & $x$ & $x$ & $x$ & $x$ & $x$ & $\checkmark$ & $\checkmark$ & $\checkmark$ & $\checkmark$ & $\checkmark$ & $x$ \\
\hline \begin{tabular}{|l|}
$\begin{array}{l}\text { in situ } \\
\text { stainings }\end{array}$ \\
\end{tabular} & $x$ & $x$ & $x$ & $x$ & $x$ & $x$ & $x$ & $\checkmark$ & $\checkmark$ & $\checkmark$ & $\checkmark$ & $x$ \\
\hline $\begin{array}{l}\text { confocal } \\
\text { scans }\end{array}$ & $x$ & $x$ & $\times$ & $x$ & $x$ & $x$ & $\times$ & $\checkmark$ & $\checkmark$ & $\checkmark$ & $\checkmark$ & $x$ \\
\hline
\end{tabular}

Figure 24: Summary of the analyses of transgenic reporter lines

Results are sorted by fragments from up- to downstream constructs. Constructs are depicted according to their color code (cf. Figures 11 and 18). Insertion analyses through amplification of the respective reporter gene with specific primers for either tGFP (six3-reporter lines) or DsRedEx (rx-reporter lines) are depicted in the row 'PCR analysis'. Results from testing the reporter gene expression are summarized in the row 'in situ stainings'. 'Promoter deletions' indicates absence for $9 \mathrm{bp}$ within the promoter construct (see chapter 3.3.2.1). Results from larval brain scans analysis for the respective reporter gene are shown in 'confocal scans'.

\subsection{Analysis of the fluorescence signal of the 'Mushroom Body' line and the 'Brainy' line}

For imaging the L1 brain, two different lines were used. One line is an enhancer trap was found in the GEKU screen and is called the 'Mushroom Body' (MB) line. A second line was created by crossing two lines, that mark glial cells of the central nervous system and neural tissue respectively, it is called the 'Brainy' line.

\section{The `Mushroom Body’ line}

The 'MB' line is an enhancer trap line with the number G 11410, in which the artificial promoter $3 \times \mathrm{xP}$ drives the fluorescent reporter gene eGFP. This construct inserted on the 4th chromosome near the Glean_07701-OG17474, which is the orthologue to Dm-spite (http://www.geku-base.unigoettingen.de/Details.aspx?MainIDResult=G11410). The molecular function of Dm-spite is still unknown, but it is coding for a hydroxyacylglutathione hydrolase and in Drosophila it is expressed in the 

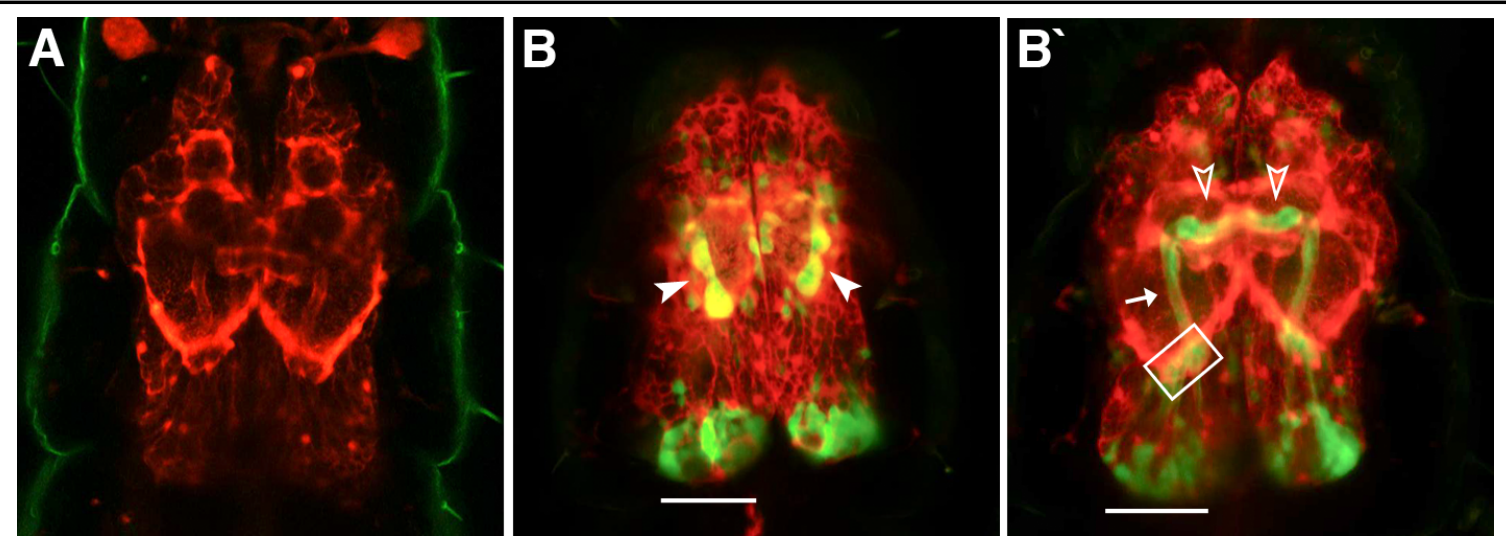

Figure 25: Characterization of the 'Mushroom Body' ('MB') line by crossing with the glial marker line 6xP3::DsRedEx.

(A) Glial cells are marked by 6xP3::DsRedEx (see chapter 1.5), depicted in red. (B and B') Crossing between the line 6xP3::DsRedEx and the 'MB' line. (B) Optical dorsal section. Vertical lobes (white arrowheads) are detected at the surface of the protocerebrum. Posterior lies a cluster of Kenyon cells (white line). (B') Optical median section. The cluster of Kenyon cells is marked by a white line (one side indicated). The calyx is indicated by a white square. The median lobes (white open arrowheads) are located in a right angle to the Pedunclus (white arrow). Anterior is oriented to the top .

somatic mesoderm (Tomancak et al., 2002; Kwon et al., 2009).

To characterize the expression pattern of the 'MB' line, it was crossed with a line expressing DsRexEx under the control of 6xP3 (chapter 1.5; Figure 25, A). Some cells positive for eGFP were spread all over the brain, but these were not further characterized. The Kenyon cells of the Mushroom Body were the most prominently marked structure posterior in the brain (white dash; Figure 25, B and B'). These cells were embedded by putative cortex glial cells, that form a net like structure, which will be referred to as the 'posterior glial tissue'. The putative Kenyon cells take up almost all of the posterior part of each brain hemisphere (white line; Figure 25, B and B'). They project to the anterior region through the posterior glial tissue into the protocerebrum. At the protocerebral neuropile border, elongated oval shapes, which represent the calyces could be detected (white square; Figure 25, B`). From here, marked axon bundles project anterior through the protocerebrum. Those bundles display the Pedunculus (white arrow; Figure 25, B'). At the end point, this structure splits into a horizontal projection forming the median lobes and into a vertical projection forming the vertical lobes. The median lobes (open arrowhead; Figure 25, B`) are parallel to the Central Body, which is located posterior to them. These lobes span almost completely across the interhemisphereic fissure. The vertical lobes (white arrowhead; Figure 25, B) are located at the surface of the protocerebrum. Thus, the larval brain comprises a Mushroom Body comparable to the adult one, which is visualized by the 'MB' line. 


\section{The 'Brainy' line}

The 'Brainy' line was generated by crossing the $6 \mathrm{xP} 3:$ :eCFP glia reporter line (chapter 1.5) with a line, which was generated in the laboratory of Michalis Averof. This second line expresses DsRedEx under the control of the regulatory region of the elongation Factor II (EFII). Additionally, this line carries the positive transfection marker 3xP3::eGFP. After respective sibling crossings, all individuals of the 'Brainy' line showed the signals for EFII::DsRedEx and 3xP3::eGFP, and partially $6 x \mathrm{P} 3:$ :eCFP. For analysis, animals which were also positive for $6 \times \mathrm{P} 3:$ :eCFP expression were chosen.

The 'Brainy' line allows to monitor the maturation of the larval brain in vivo. The developing embryonic structures and developing neuromeres were identified and named in their correlation to the morphology and position of neuropiles within the first larval instar brain.

The earliest expression of 6xP3::eCFP was detected in a 17h old embryo. Here, weak expression was detected in some cells, while expression of EFII::DsRedEx was not found at this time (data not shown). A similar pattern was visible in $24 \mathrm{~h}$ old embryos. Here, faint expression of DsRedEx was detected (Figure 26, A). Both signals became stronger during ongoing development of the embryo. At an age of 30h an increasing number of cells expressed either eCFP or DsRedEx (Figure 26, B). Cells positive for the 6xP3::eCFP signal showed a strong branching in 40h old embryos. Cells positive for DsRedEx were detected within the branching tissue and are probably developing glial cells (Figure 26, C). An area free of signal appeared at an age of 45h, surrounded by cells positive for eCFP (white arrowhead; Figure 26, D) and increased to typical protocerebral shape and volume in later stages. A paired central structure was detected at an age of 55h, also surrounded by an eCFP signal (open arrowhead; Figure 26, F). Dorsally of this structure, the median lobes of the Mushroom Body and posterior to this structure, the Central Body was detected in later stages. The DsRedEx signal increased and was located in putative cell bodies of neural cells (Figure 26, F-I). In 60h old embryos, Antennal Lobes (AL, asterisks) were detected anterior to the protocerebral neuropile (Figure 26, G). Posterior to the paired central structure, the first faint and unpaired structure connecting the hemispheres was detected (white arrow; Figure 26, H), surrounded by cells positive for eCFP signal. At an age of $64 \mathrm{~h}$, cells expressing 6xP3::eCFP coated this structure, representing the developing Central Body. The latter could be clearly recognized at an age of $72 \mathrm{~h}$, where cells expressing a strong eCFP signal were covering this neuropile (Figure 26, I). At this stage, the first glial cells positive for the eCFP signal surrounded the Pedunculus of the Mushroom Bodies in the posterior dorsal part of the brain (not shown). Therefore, the 'Brainy' line represents a valuable tool to visualize the development and presence of neuropiles within brains of embryos to the first larval 

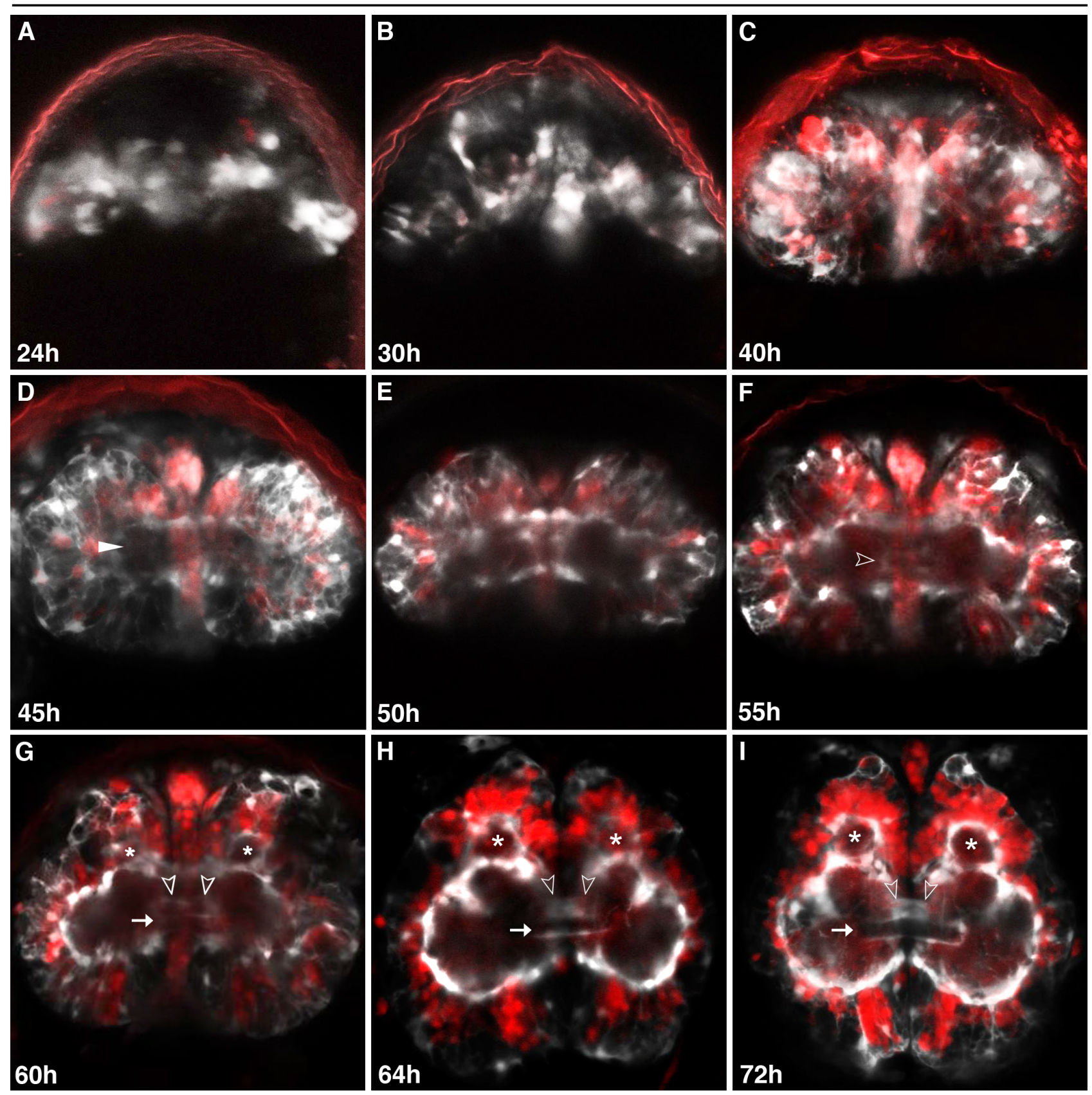

Figure 26: Embryonic developmental series of the fluorescence signal in the 'Brainy' (BA) line.

The 6xP3::eCFP (glial cells) signal is shown in white. EFII::DsRedExpress (neural cells) is depicted in red. Anterior is oriented to the top. The pace of embryonic development depends on the temperature, the indicated time relates to development at $32^{\circ} \mathrm{C}$. (A) A $24 \mathrm{~h}$ old embryo. The $6 \mathrm{xP} 3:$ :eCFP signal is weak in some cells. The EFII::DsRedExpress signal is beginning to appear in very few cells. (B) A 30h old embryo. (C) A 40h old embryo, where the 6xP3::eCFP signal is expressed in glial cells and starts to form a mesh like structure. (D) A 45h old embryo, where the putative Protocerebrum starts to form, marked by the absence of a 6xP3 signal (white arrowhead). (E) A 50h old embryo, where the shape of the protocerebrum is visible. (F) A 55h old embryo, where a paired structure is visible (open arrowhead). (G) A 60h old embryo. The Antennal Lobes (white asterisks) and the developing Central Body (white arrow) become visible (H) A 64h old embryo. (I) A 72h old embryo, where the Central Body is coated by eCFP positive cells. Asterisks mark the developing Antennal Lobes. Open arrowheads point to paired median structures, where finally the median lobes of the Mushroom Bodies appear. White arrows point to the forming Central Body. 
instar brain.

Taken together, the ' $\mathrm{MB}^{\prime}$ line as well as the 'Brainy' line are prerequisites for the following analyses of various genes in the context of embryonic brain development (second part of this work). 


\section{Results Part II: The genetic network of Central Body development}

Little is known about the genes that give identity to the neural lineages, which in turn form and develop neuropiles in the brain. The same holds true for the Central Complex, which is a very prominent central structure within the insect brain. In Schistocerca, this structure is formed during embryonic stages, while in Drosophila, it develops partially in the last larval stage and is completed during the puparium. However, in both animals the corresponding NBs, which are involved in forming this neuropile, have been identified (Willams et al., 2005; Williams et al., 2008; Boyan et al., 2010; Itzergina et al., 2009). Yet, the underlying genetic mechanisms are still unknown. The regions of the brain, from which the Central Complex develops, are known in Schistocerca and Drosophila. On this basis, genes which are expressed in homologue regions in Tribolium castaneum were identified. These genes were analyzed with respect to their role in Central Body formation in Tribolium castaneum.

\subsection{Tc-rx knock down leads to a split Central Body}

The homeobox gene Tc-rx was chosen for functional analysis, because it is expressed close to the region, where the putative progenies of the Central Body originate. Furthermore, it is also expressed close to the region, where the putative Mushroom Body NBs arise (Hein, 2007). The onset of Tc-rx expression in embryos occurs during elongation, which correlates to embryos approximately $12 \mathrm{~h}$ old at $32^{\circ} \mathrm{C}$ (Posnien, 2009; chapter 3.3.1). Additionally, its expression pattern is exclusively found in the head. Both the late onset and the exclusive head expression pattern indicate a brain specific differentiating function of Tc-rx. Especially, since knock down experiments in previous studies revealed only a weak cuticle phenotype. I.e. the labrum was reduced in size and bristles of the clypeus quartet were missing after pupal dsRNA injection of Tc-rx (Posnien, 2009), whereas the latter was not detected in this work. Taken together, all these findings regarding $T c-r x$ suggest a role in brain development.

In order to analyze the function of $T c-r x$, pupal knock down was performed with different concentrations of dsRNA in the 'Brainy' line $(1$ or $4 \mu \mathrm{g} / \mu \mathrm{l})$. The pupae injected with $4 \mu \mathrm{g} / \mu \mathrm{l}$ dsRNA died, probably due to the high dsRNA concentration. Offspring of the pupae injected with 1 $\mu \mathrm{g} / \mu \mathrm{l}$ were analyzed by confocal laser microscopy scannings. Normally, the Central Body (white arrow; Figure 27, A) spans the interhemispheric fissure. At this stage, it is the only neuropile which connects the brain hemispheres. The median lobes of the Mushroom Body (white arrowhead; Figure 27, A) are located anterior to this prominent neuropile. This was also the case in the RNAi brains, where the median lobes were still found anterior to the Central Body (white arrowhead; 
Figure 27, B). The Central Body, however, was split and had a short oval form (white arrow; Figure 27, B). Also, it did not span the interhemispheric fissure. This phenotype was detected in three of nine larvae. Interestingly, the non scanned siblings were able to hatch and to molt. Also, one second larval instar brain with split Central Body was scanned.

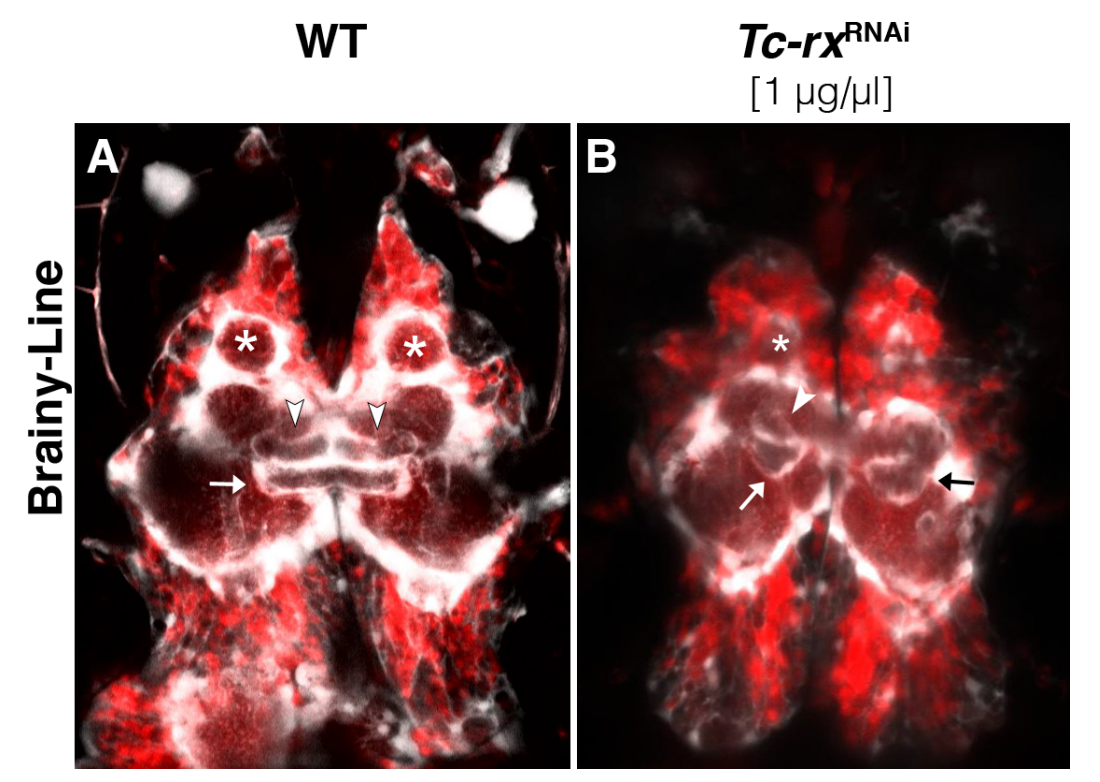

is depicted in white. Anterior is oriented to the top.
Figure 27: Knock down of Tc-rx leads to a split Central Body in first larval instar brains.

(A) The 'Brainy' line native situation. (B) Split Central Body (CB) after knock down of Tc-rx. Separation of the median (white arrowhead) lobe of the Mushroom Body (MB) and CB (white arrow) for the left hemisphere. The black arrow points out the same area, but a dsicrimination between $\mathrm{MB}$ and $\mathrm{CB}$ was not possible due to technical reasons. White asterisks: Antennal Lobes; white arrowhead: median lobes of the MB; white arrow: CB. For a better contrast the eCFP signal

However, the described results are preliminary and need to be verified by a second independent knock down experiment for $T c-r x$. Apparently, $T c-r x$ is required for a process mediating the crossing of the brain midline. I.e. Tc-rx is suggested to play an important role in establishing the resepective midline signaling for a proper Central Body formation (see chapter 5.4.2), but the split Central Body phenotype after Tc-rx RNAi is very likely to be due to secondary effects. Since the focus was to identify genes that influence the Central Body development in Tribolium as such, further analysis of $T c-r x$ regarding was not performed here, especially since the Central Body was missing after $T c$ six3 RNAi (chapter 4.3.3).

\subsection{Tc-otd1 is not the antagonist of $T c$-six 3 in Central Body growth}

In earlier studies, it was hypothesized that Tc-six3 and Tc-otdl are antagonists in anterior head patterning during embryonic stages (Posnien; 2009). A knock down of Tc-six3 led to absence of the larval Central Body (see chapter 4.3.3) and to an expansion of the Tc-otdl expression domain. This was visualized in whole mount in situ hybridization in respective embryos and suggested that $T c$ six 3 represses $T$ c-otd 1 in the neuroectoderm. Based on the non overlapping expression patterns, a 
negative feedback loop was suggested. Thus, it was hypothesized, that a knock down of Tc-otdl could enlarge the areas of Tc-six3 expression, and vice versa. Due to the role of Tc-six3 in Central Body development, it was hypothesized, that a knock down of Tc-otd1 would lead to an enlargement of this neuropile.

It has previously been described, that a pupal Tc-otd1 knock down leads to severe phenotypes and to high cellular lethality during blastodermal stages of the offspring (Kotkamp et al., 2010). This is congruent with the previously described "early regionalization" function of Tc-otdl in blastodermal stages (Schinko et al., 2008). Therefore, embryonic injections were performed to overcome these early regionalizing defects and to asses the late function determining the neurogenic tissue. By embryonic injections with $1 \mu \mathrm{g} / \mu \mathrm{l}$ dsRNA into staged egg lays, a phenotype with weak to intermediate defects should be generated. Therefore, embryos aged $7-8 \mathrm{~h}$ and $8-9 \mathrm{~h}$ at $32{ }^{\circ} \mathrm{C}$ were injected. Larvae with weak phenotype showed a reduction in size of one hemisphere (indicated by white lines on different levels, Figure 28, B). Neuropiles like the Central Body, the Mushroom Body, and the Antennal Lobes showed no obvious defects. The protocerebrum and the posterior glial tissue were shortened along the anterioposterior axis. In case of intermediate phenotypes, both brain hemispheres were reduced in length. The Antennal Lobes, the Central Body and the Mushroom Body (out of focus in ; Figure 28, C) appeared to be unaffected (Figure 28, C).
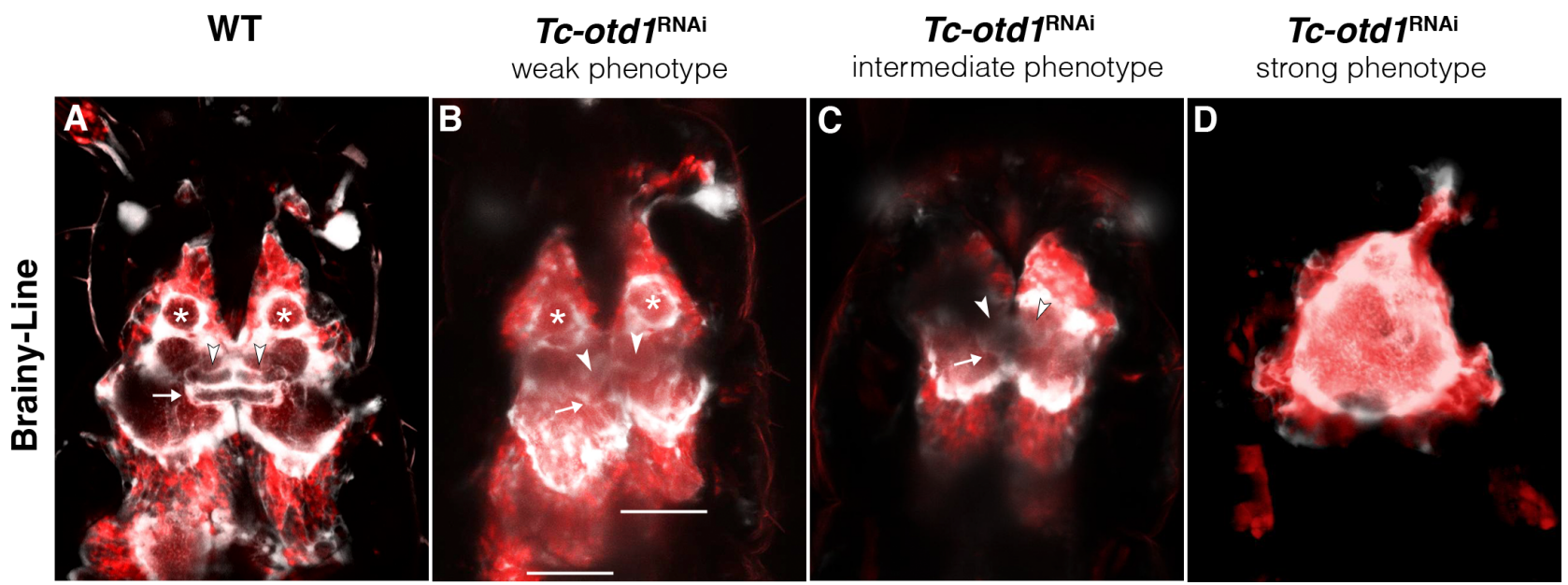

Figure 28: Knock down of Tc-otd1 leads to a reduction of brain size.

Embryonal injections of $1 \mu \mathrm{g} / \mu \mathrm{l}$ were performed in embryos $7-8 \mathrm{~h}$ old at $32^{\circ} \mathrm{C}(\mathrm{A})$ 'Brainy' line native situation. (B) Weak phenotype of Tc-otdl knock down, where the right hemisphere is reduced; white lines indicate the end of posterior glial tissue. (C) Intermediate knock down phenotype, where both hemispheres are reduced. (D) Strong knock down phenotype, which leads to unstructured brain mass. White asterisks: Antennal Lobes; white arrowhead: median lobes of the Mushroom Body; white arrow: Central Body; white line: posterior end of the posterior glial tissue. Anterior is oriented to the top. 
In larvae with strongly affected brains, the brain architecture was destroyed in a way that no neuropiles could be identified anymore (Figure 28, D).

The brain defects were correlated to the time slot of dsRNA injection, hence a knock down of Tcotdl in young embryos caused stronger effects than a knock down in later stages (Figure 29). In the embryo batch injected at $7-8 \mathrm{~h}$ at $32{ }^{\circ} \mathrm{C}$, strong phenotypes occurred in $42,1 \%$, while weak and intermediate phenotypes, meaning those with reduced brain hemispheres, occurred in $21,1 \%$ (Figure 29, 7-8h bar graphs). The strong brain phenotype occurred together with deletions of the dorsal head structures (compare Schinko et al., 2008). Injections into older embryos revealed no strong brain phenotypes, whereas weak brain phenotypes were predominant with 62,5\% (Figure 29; 8-9h bar graphs).

Correlation between the point in time of Tc-otd $1^{\mathrm{RNAi}}$ and the repective brain phenotype

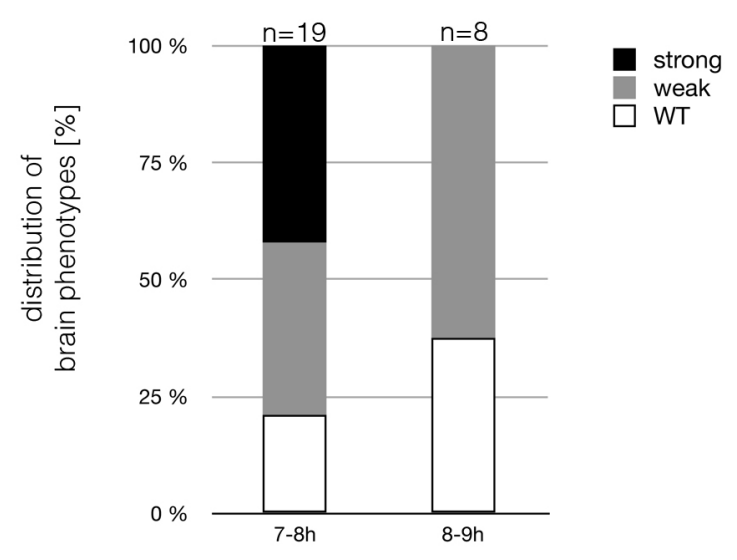

Figure 29: Correlation between the point in time of Tc-otd1 RNAi and resulting brain phenotypes - early knock down leads to strong phenotypes.

The first bar shows the distribution of phenotypes detected in embryos injected with $1 \mu \mathrm{g} / \mu 1$ Tc-otd 1 dsRNA at $7-8 \mathrm{~h}$ old at $32^{\circ} \mathrm{C}$; wild type (WT): $21,1 \%$; weak phenotype (one or both hemispheres reduced): $36,8 \%$; strong phenotype (brain unstructured tissue): 42,1\%. The second bar shows distribution of detected phenotypes within embryos injected with $1 \mu \mathrm{g} / \mu \mathrm{l}$ Tc-otd 1 dsRNA at $8-9 \mathrm{~h}$ old at $32^{\circ} \mathrm{C}$; WT: $37,5 \%$; weak phenotype: $62,5 \%$; strong phenotype: none.

In order to quantify the differences in neuropile size, 5 embryos of the injection performed after $8-9 \mathrm{~h}$ at $32{ }^{\circ} \mathrm{C}$ with intermediate phenotypes (cf. Figure 28, C) were analyzed regardng their relative brain size in correlation to the size of the head capsule. Only embryos where brain and head capsule were almost parallel were selected. There, different tissues and neuropiles were measured in length and width. The resulting data were normalized to either the head capsule length or to the cervix width. For the head capsule length (line a; Figure 30) the distance between the antenna basis to the cervix fold ('neck') was measured. For the head capsule width (line b; Figure 30), the distance from the most distant to the most lateral points of the cervix fold was measured (yellow lines in, Figure 30). For measuring length and width of brain tissues and neuropiles the most distant points were measured (Figure 30).

The bar graph in Figure 30 shows the arithmetical mean of all measured structures and neuropiles for WT and RNAi treated individuals. A students T-test was performed to verify statistical significance. Hence, the observed differences in brain length and width and also in protocerebrum 
width were significant. No significant difference were found regarding the size of the Central Body. Thus, the hypothesis, that a knock down of Tc-otd1 leads to an enlargement of the Central Body could not be confirmed here.

\section{Correlation between head capsule and brain size after Tc-otd $1^{\mathrm{RNAI}}$}

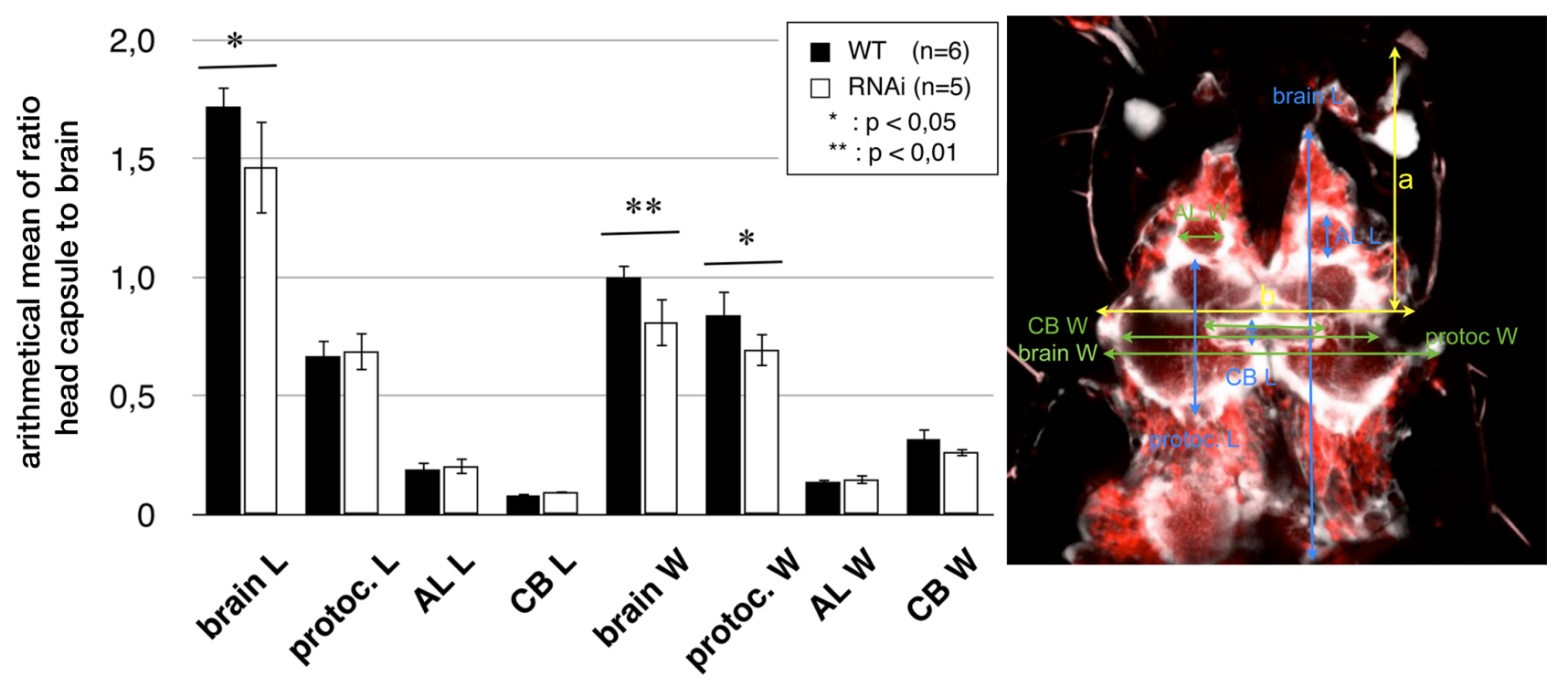

Figure 30: Correlation between head capsule and brain size after Tc-otd1 knock down.

Embryonic knock down with $1 \mu \mathrm{g} / \mu \mathrm{l}$ Tc-otdl dsRNA at $8-9 \mathrm{~h}$ at $32^{\circ} \mathrm{C}$ was performed in the 'Brainy' (BA) line. Bar graph: the $\mathrm{X}$-axis indicates different neuropiles which were measured in length (L) and width (W) for wild type (WT) and RNAi treated first instar larvae. The y-axis indicates the arithmetical mean of measured neuropiles in correlation to the head capsule. An unpaired and two-sided student's T-test was performed testing the statistical significance. Significant results are indicated by one asterisk $(\mathrm{p}<0,05)$; highly significant results are indicated by two asterisks $(\mathrm{p}<$ 0,01). The right panel shows the untreated BA situation (WT); green lines indicate start and end point of measured width; blue lines indicate start and end point of measured length; yellow lines indicate the measured width and length of the head capsule. (a) measurement for head capsule length starting at the antenna basis and ending at the cervix. (b) measurement for the head capsule width was performed at the farthest lateral points of the cervix. protoc.: protocerebrum; AL: Antennal Lobes; CB: Central Body; L: length; W: width.

\section{3. $T c$-six 3 and $T c-c h x$ are required for Central Body formation}

The NBs, which contribute to the Central Body formation, are located in anterior median regions of the brain in Schistocerca and Drosphila (Willams et al., 2005; Williams et al., 2008; Boyan et al., 2010; Itzergina et al., 2009). Thus, a screen for candidate genes, which are expressed in the respective regions in Tribolium castaneum, was performed. Thereby, the genes Tc-six3 and Tc-chx were found (Posnien, 2009). From their expression pattern, it was hypothesized, that these genes play major roles in Central Body formation in Tribolium castaneum. First, in order to clarify this, 
the expression patterns of $T c$-six 3 and $T c-c h x$ were analyzed for an overlap of their expression domains by double in situ hybridization experiments.

\subsection{1 $T c-c h x$ and $T c$-six 3 coexpression analysis}

Staged embryos of $0-24 \mathrm{~h}$ and $24 \mathrm{~h}-48 \mathrm{~h}$ at $32^{\circ} \mathrm{C}$ were stained with probes for Tc-six3 and Tc-chx. While Tc-six3 expression started early in blastodermal stages (Posnien, 2009), Tc-chx was first expressed in embryos in elongating stages (Figure 31, A). Here, it was expressed in a small roundish pattern anterior lateral and partially overlapping with expression of Tc-six3 (Figure 31, $\left.\mathrm{A}^{\prime}\right)$. The expression within this group of cells increased during ongoing elongation (Figure 31, B). A slightly longish and faint expression spread to the median tissue (black arrow; Figure 31, B'), later developing into basal expression within the labrum. In fully elongated embryos, this basal domain in the labrum was weaker than the expression lateral of the anterior median head tissue (black arrow; Figure 31, C'). In fully elongated embryos, an additional domain posterior in the rim of the head lobes, where the expression patterns of Tc-six3 and Tc-chx were overlapping, was also detected (black arrowhead; Figure 31, C'). In embryos aged $24 \mathrm{~h}$ at $32^{\circ} \mathrm{C}$, the expression pattern of Tc-chx expanded slightly in all directions, especially the anterior domain (Figure 31, D). This domain almost completely covered the lateral expression domain of Tc-six3 (open arrow; Figure 31, D'). The expression domains in the putative eye anlage were still overlapping, but $T c$-chx overgrew Tc-six3 (black arrowhead; Figure 31, D'). The posterior part of the Tc-chx expression domain lateral to the labrum basis still showed a slight expression of Tc-six3 (black arrow; Figure 31, D'). However, in later stages the weak expression connecting the lateral expression domain and labrum basis could not be detected anymore (open arrowhead; Figure 31, E`). Furthermore, a small thin band of cells positive for only $T c-c h x$ expression in the posterior part of the lateral expression domain of Tc-chx was detected (white arrow; Figure 31, E'). The expression patterns of Tc-chx and Tc-six3 were still overlapping in the putative eye anlage (black arrowhead; Figure 31, E'). Until this stage, Tc-chx expression was located exclusively in the head (Figure 31, E), whereas additional domains positive for $T c-c h x$ arose later, also within other regions of the body. 
A

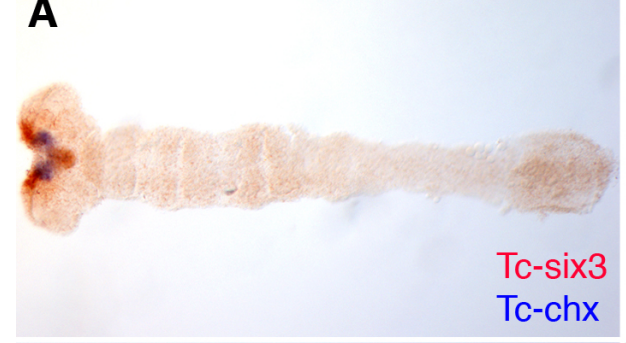

B
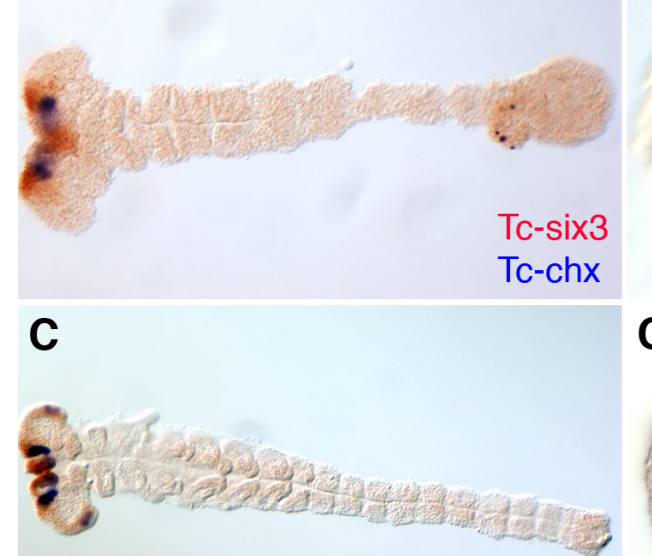

Tc-six 3

Tc-chx

D

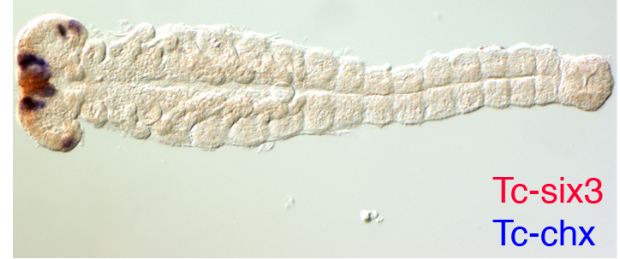

E

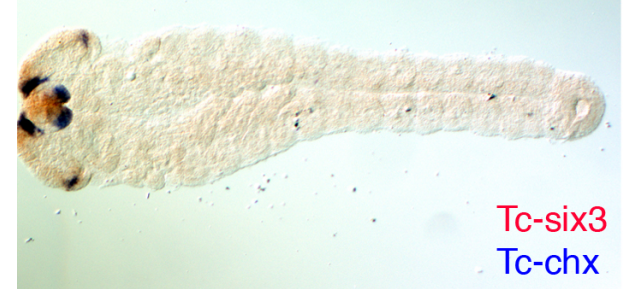

A

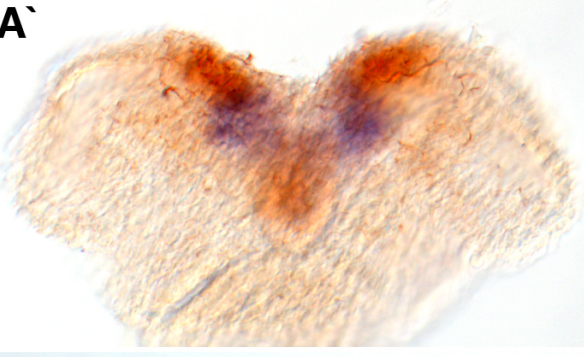

B'

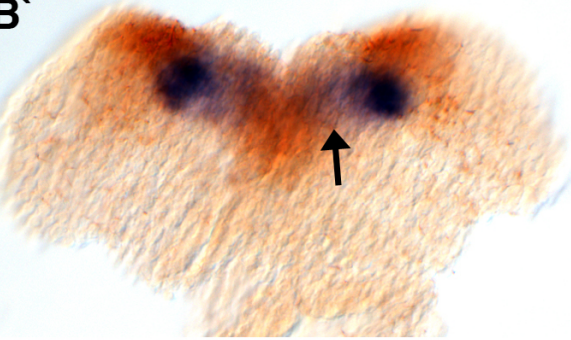

C

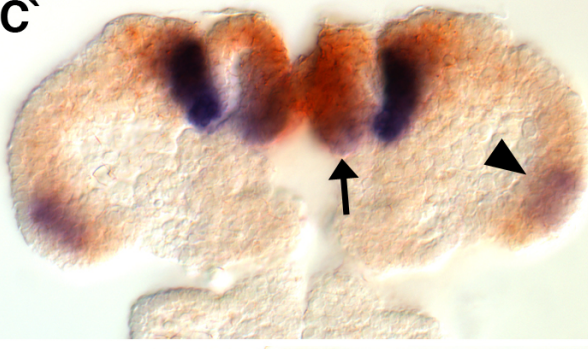

D'

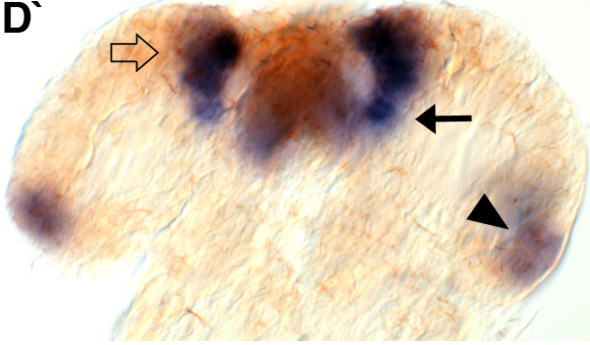

E'

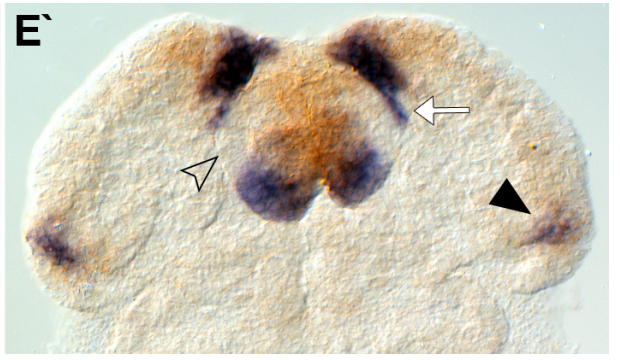

Figure 31: Double in situ hybridization of $T c-c h x$ and $T c$-six3 demonstrates an overlapping expression pattern in up to $24 \mathrm{~h}$ old embryos.

(A-E) Ventral view of embryos, anterior facing to the left. (A'-E') Close up of the head, anterior facing up. $T c$-chx is depicted in blue and $T c$-six 3 in red. (A and B) Approximately $13-14 \mathrm{~h}$ old embryos. (A) shows a younger embryo than the embryo depicted in (B). (A') Tc-chx expression is detectable in a dotted domain. (B') A lateral domain with strong expression of $T c-c h x$ and a longish domain developing into labral expression in later stages (black arrow). (C) $18 \mathrm{~h}$ old embryo. (C') The pointshaped domain had stretched out anterior, faint expression in the putative eye anlage (black arrowhead). The basal labral expression is indicated by a black arrow. (D) $24 \mathrm{~h}$ old embryo. (D') The domain lateral to the labrum has a strong expression (open

arrrow), its posterior part shows still a coexpression with $T c$-six3 (black arrow). (E) 26h old embryo. (E') The lateral domain and posterior expression of the labrum detach from each other (open arrowhead). Tc-chx shows a longish expression lateral to the labrum (white arrow). $T c$-six 3 is only weakly expressed. The black arrowheads indicate the overlapping expression pattern in the putative eye anlage. All indicated ages correlate to the development at $32^{\circ} \mathrm{C}$.

To analyze the expression pattern of $T c$-chx at later stages, single in situ hybridization was performed in embryos staged $24-48 \mathrm{~h}$ and $48-72 \mathrm{~h}$ at $32{ }^{\circ} \mathrm{C}$.

A segmental expression pattern arose de novo and spread out stepwise from the mandibular to the abdominal segments. More domains arose laterally in the head lobes, while the previous expression 
remained. In embryos aged 40-55h, the segmental expression was finally detected in all segments (Figure 32, C). The head expression domains remained unchanged, i.e. the superficial expression median in the head (black arrow; Figure 32, B), the posterior domain in the rim of the head forming
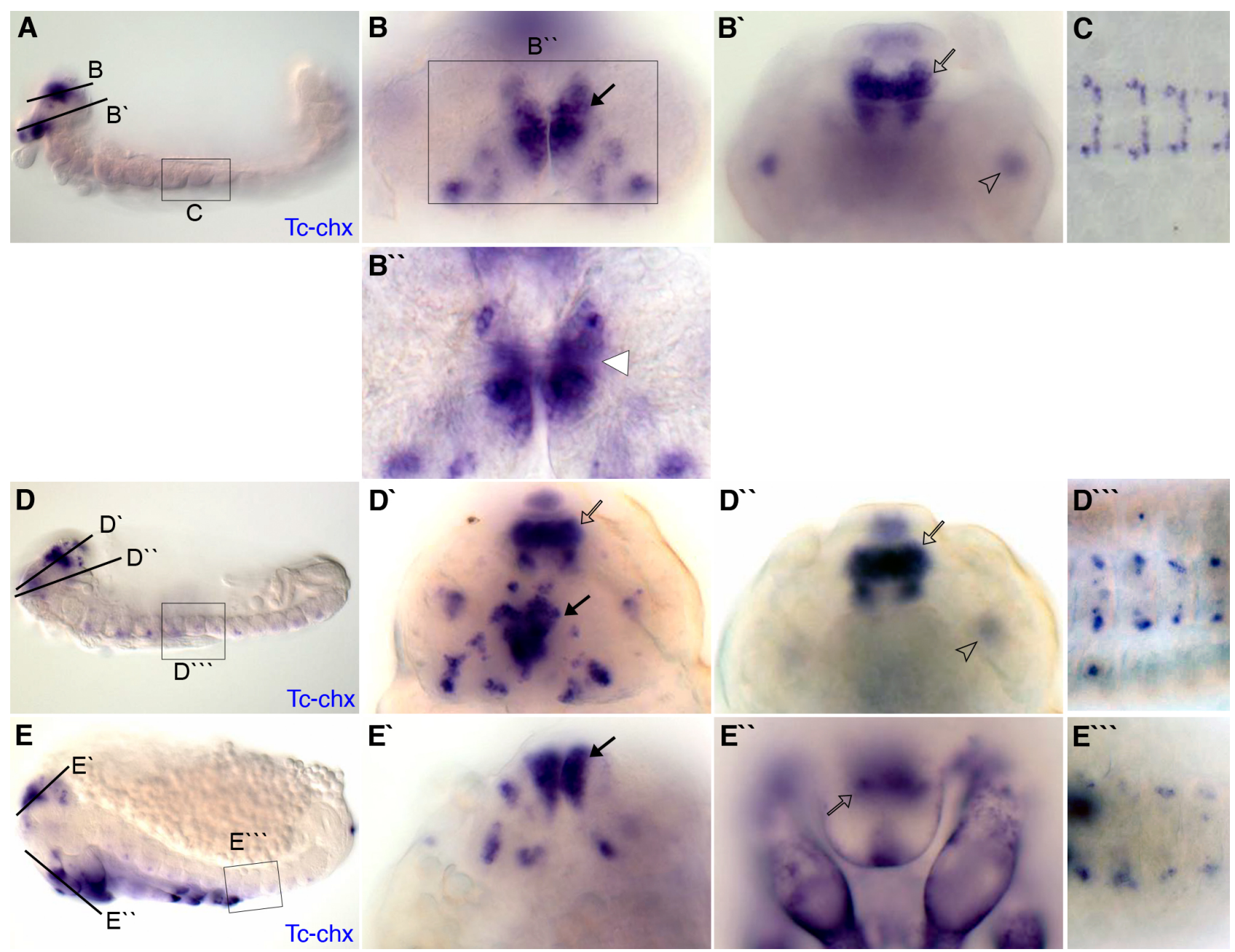

Figure 32: Tc-chx expression pattern.

(A, D, E) Lateral view, anterior facing left.(B, B', D', D", E', E") Close ups of various optical sections in the head indicated by lines in $\mathrm{A}, \mathrm{D}$, and $\mathrm{E}$ respectively, anterior facing up. $\left(\mathrm{C}, \mathrm{D}^{\cdots}, \mathrm{E}^{\cdots}\right)$ Indicated by squares in $\mathrm{A}, \mathrm{D}$, and $\mathrm{E}$ respectively; ventral view of abdominal segments, anterior facing left. All images were prepared in bright field, except B", which was prepared with a Nomarski-prism. The black arrow indicates the median expression domain, the open arrow indicates the labral expression domain, the open arrowhead indicates the putative larval eye expression domain in the respective images. (A) An embryo 40-55h old. (B) Close up of the dorsal expression domain of different embryos at similar stage. (B") Same expression domain in Nomarski-optics, midline crossing structures are detectable (white arrowhead). (B') The labral expression domain and faint lateral domains in the developing larval eyes. (C) Segmental expression pattern of different embryos at similar stages, here the abdominal segments A1-A4 are depicted. (D) An embryo 50-60h old. (D') Complex expression pattern at the dorsal head. (D") The labral expression from the ventral view with faint lateral domains. (D ") The segmental expression pattern decreases, abdominal segments A1-A4. (E) An embryo older than 65h. (E') Expression domain in the dorsal head. (E”) Frontal view of the labral domain. (E”) Weak segmental expression pattern in abdominal segments A1-A4. All indicated ages correlate to the development at $32^{\circ} \mathrm{C}$. 
the larval eyes (open arrowhead; Figure 32, B'), as well as the labral expression (open arrow; Figure 32, B'). Through analysis with a DIC filter, interhemispherical projections could be detected on the level of the median strong expression domain of Tc-chx (white arrowhead; Figure 32, B“'). The expression pattern in the head became more complex (Figure 32, D'). While the median expression domain remained constant (black arrow; Figure 32, D'), more cells and clusters in the surrounding tissue expressed Tc-chx (Figure 32, D'). The labral expression (open arrow; Figure 32, D") as well as the eye expression domain of $T c-c h x$ remained unchanged (open arrowhead; Figure 32, D“), whereas the segmental expression domain diminished with time (Figure 32, D“'). Here, the lateral most domains remained constant in their expression level, while the median domain was hardly detectable (Figure 32, D“').

At an age of approximately $65 \mathrm{~h}$ at $32^{\circ} \mathrm{C}$, cuticle was secreted by epidermical cells. This leads to strong background stainings, especially in the head and appendages (Figure 32, E). However, the overall expression pattern in the head was found to remain unchanged with its median expression domain and single spots surrounding it (Figure 32, E'). The labral expression was still detectable, although in slightly changed shape (open arrow; Figure 32, E“). The segmental expression strongly decreased and only the lateral domains were detectable (Figure 32, E“'”).

Taken together, $T c$-chx became expressed in the ventral tissue of the abdomen and also in a more complex manner in the head after an age of $24 \mathrm{~h}$. Since Tc-six3 remains expressed only in the anterior part of the embryo, the coexpression pattern of $T c-c h x$ and $T c-s i x 3$ is suggested to be limited in time and location. However, during the $0-24 \mathrm{~h}$ slot, a colocalization of $T c-c h x$ and Tc-six 3 was found for a restricted area in the anterior head region. Therefore, the amount of NBs arising in the $T c$-chx positive ectodermal region was analyzed next, especially since they are a subset of $T c$ six3 positive NBs.

\subsubsection{At least 4 NBs delaminate from the $T c$-chx positive anterior neuroectoderm}

For the quantification of the NBs, that delaminate from the Tc-chx positive domain, double in situ hybridziations with staged egg lays of the SB-strain were performed at an age of 12-18h, 18-24h, and 24-30h. Analysis was performed with antisense probes for Tc-chx and Tc-ase as a NB marker. The first NB arising in the $T c-c h x$ expression domain was detected in elongating embryonic stages at approximately 13-14h of age (Figure 33, A`). Two additional NBs arose in fully elongated embryos (Figure 33, B'). In later stages, a maximum of four NBs was identified within the Tc-chx expression domain (Figure 33, D`and E'). At later stages, it was not possible to match NBs with the overlaying ectoderm, due to the morphological separation of neural from epidermal tissue. 
Therefore, the detected number of NBs is a minimum number, as. it is very likely, that more NBs, which are $T c-c h x$ positive, arise.

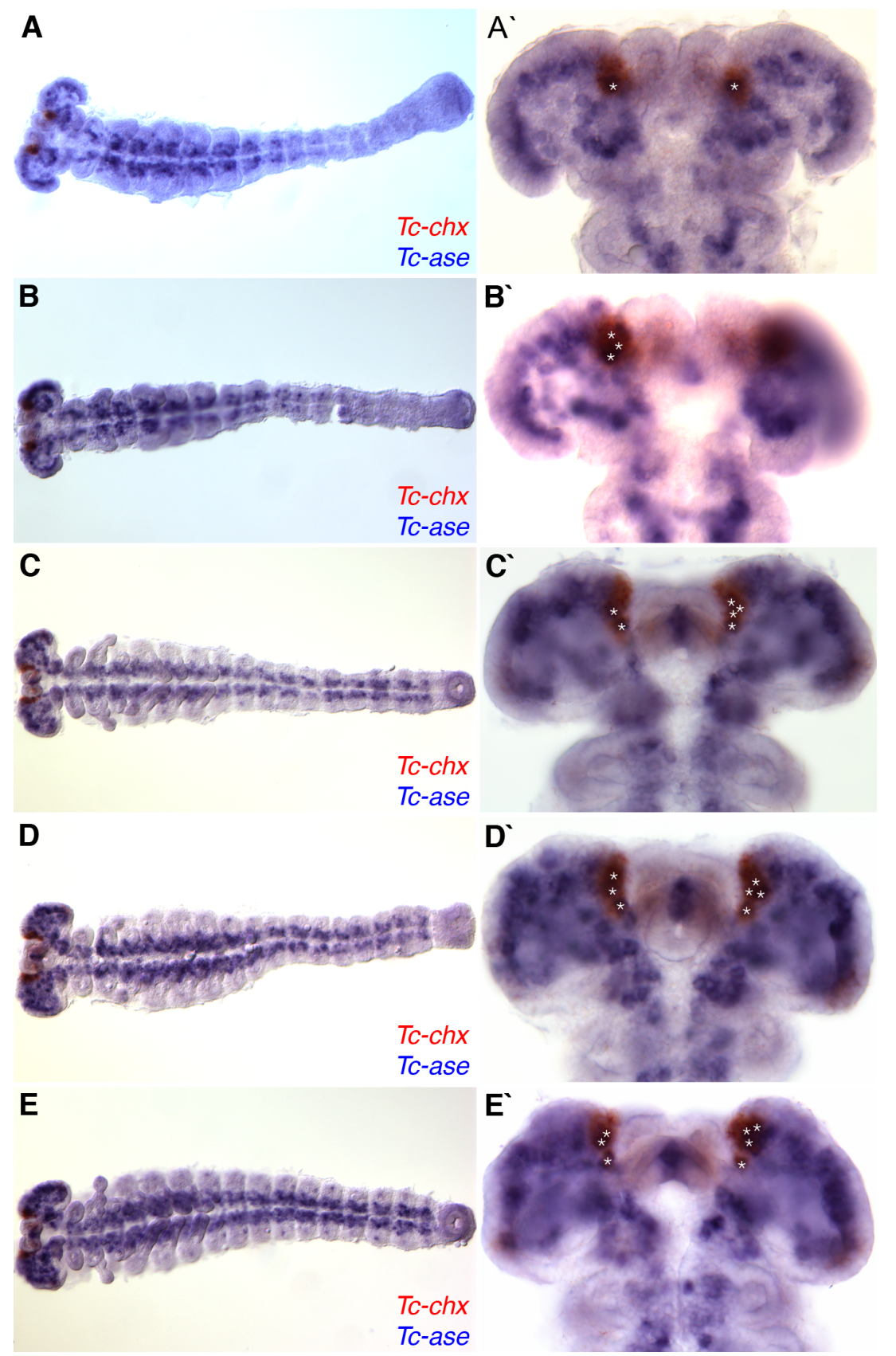

Figure 33: The number of NBs positive for $T c-c h x$ varies in similarly aged embryos.

Staging of embryos was performed at $32^{\circ} \mathrm{C}$. (A-E) Embryos are oriented facing anterior to the left. (A'-E') Close up of the head, anterior facing up. NBs are marked by white asterisks. (A) Elongating embryo, approximately 13-14h old. (A') One Tc-chx positive NB per hemisphere. (B) Almost fully elongated embryo, approximately $18 \mathrm{~h}$ old. (B') 3 NBs per hemisphere (right hemisphere out of focus). (C) Fully elongated embryo, approximately $20 \mathrm{~h}$ old. (C') 2 NBs in the left and 4 NBs in the right hemisphere. (D) Fully elongated embryo, approximately $24 \mathrm{~h}$ old. (D') $3 \mathrm{NBs}$ in the left and 4 in the right hemisphere. (E) Embryo in retraction movement, approximately 26h old. (E') 3 NBs in the left and 4 NBs in the right hemisphere.

The number of NBs within one developmental stage was found to be dynamic, rather than stereotypic. In some cases, older embryos had less NBs than younger ones (compare Figure 33, B` and $C^{\prime}$ ). However, a maximum number of 4 NBs positive for $T c$-chx expression could be detected until an age of approximately $26 \mathrm{~h}$ at $32^{\circ} \mathrm{C}$.

From the coexpression analysis of $T c-c h x$ and $T c-\operatorname{six} 3$ (chapter 4.3.1) it is known that the identified NBs were positive for both genes, i.e. Tc-chx positive NBs are a subset of those positive for Tc-six3. 


\subsection{3 pRNAi of $T c$-six3 and $T c$-chx in brain imaging lines led to loss of the Central Body}

After the quantification of the number of the NBs positive for Tc-six3 and Tc-chx within the neuroectoderm, the following questions arose: Which influence do these genes have on the development of the brain and which neural fate is determined by their expression in the NBs? To answer these questions, RNAi experiments starting with pupal injections of $T c$-six 3 dsRNA were performed.

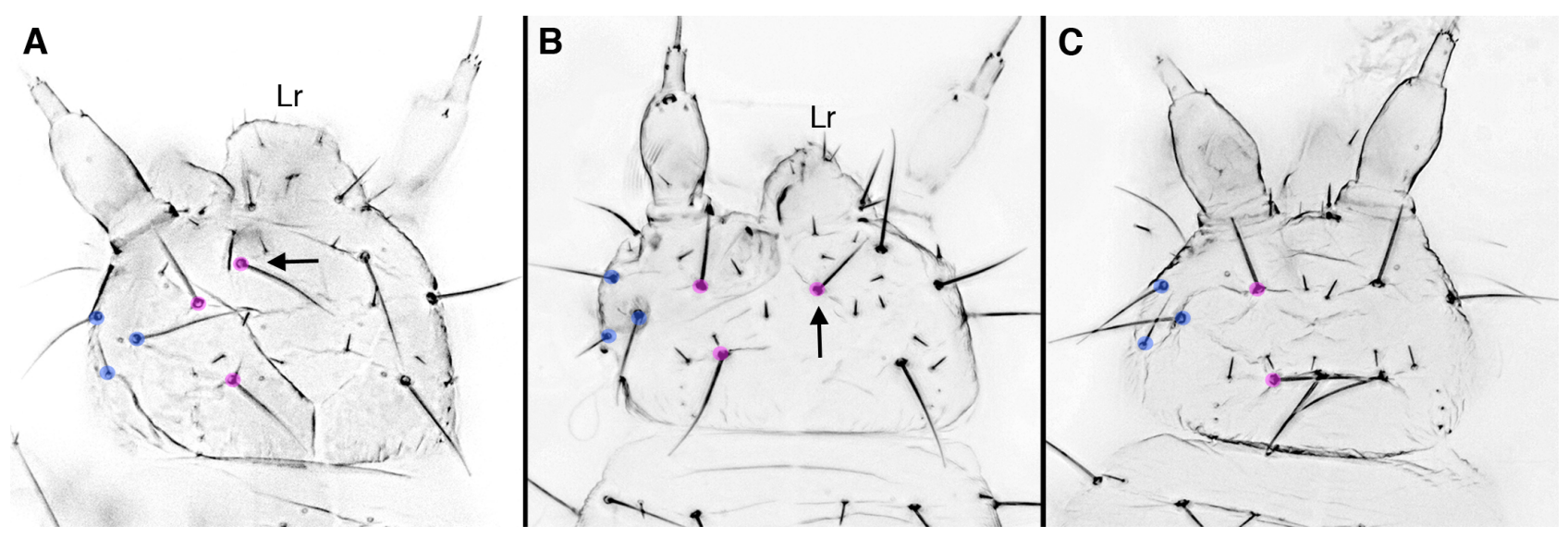

Figure 34: Tc-six3 cuticle RNAi phenotypes

(A) WT cuticle. (B) Weak cuticle phenotype after injection of $100 \mathrm{ng} / \mu 1 \mathrm{Tc}$-six3 dsRNA. The labrum (Lr) is slightly affected and the anterior vertex seta is displaced (black arrow). (C) Strong cuticle phenotype after injection of $1 \mu \mathrm{g} / \mu \mathrm{l}$ $T c$-six 3 dsRNA. The $\mathrm{Lr}$ and the median head tissue including the anterior vertex seta are missing. All heads are depicted by a dorsal lateral view, facing anterior up. Gena seta are marked blue. Vertex seta are marked pink. Black arrows point to anterior vertex seta.

It was known from prior studies, that $T c$-six3 RNAi causes severe cuticle phenotypes when injecting highly concentrated dsRNA, i.e. $1 \mu \mathrm{g} / \mu \mathrm{l}$ and higher. Therefore the efficiency of the RNAi treatment was monitored by analysis of the head cuticle. Indeed, the anterior median tissue of the head, including the labrum was deleted using $1 \mu \mathrm{g} / \mu \mathrm{l}$ of dsRNA (Figure 34, C). Less concentrated dsRNA, here $100 \mathrm{ng} / \mu \mathrm{l}$, caused a malformation of the vertex seta and the bristle pattern on the dorsal head (Schinko et al., 2008; Posnien, 2009) as well as the labrum (Figure 34, B).

Functional analysis of Tc-six3 regarding its role in brain development was initially performed with injections of $100 \mathrm{ng} / \mu \mathrm{l}$ dsRNA in female pupae of the 'Brainy' line. The brains of the RNAi treated offspring were similar to those of wildtype in size and shape. The posterior glial tissue was not affected. The Antennal Lobes (asteriks, Figure 35, B) and the median lobes of the Mushroom Bodies were present (white arrowhead, Figure 35, B). However, the position of the latter was 


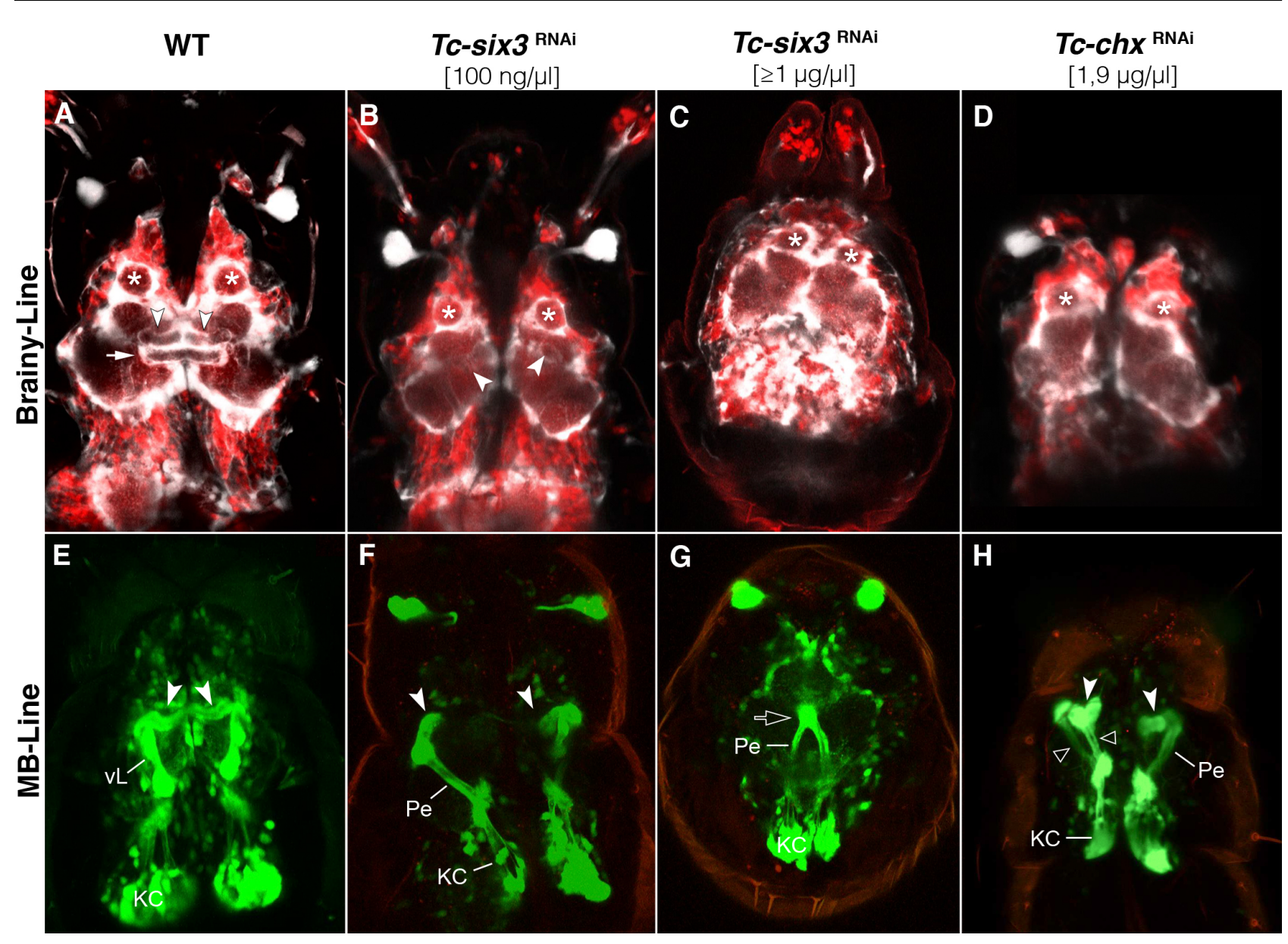

Figure 35: Tc-six3 and Tc-chx knock down leads to a loss of the Central Body.

Larval offspring of animals treated with pRNAi for $T c$-six3 and $T c-c h x$. Larvae are presented with anterior to the top. (A-D) Larvae with various RNAi defects in the 'Brainy' (BA) line background, the 6xP3::eCFP signal is depicted in white. (E-H) Larvae with various RNAi defects in the 'Mushroom Body' ('MB') line background. The Antennal Lobes are marked with white asterisks, the median lobes of the Mushroom Bodies (MB) are pointed out by white arrowheads. Kenyon cells are indicated by KC and the Pedunculus by Pe. WT with typical expression patterns of the BA line is shown in (A), and of the 'MB' line in (E) respectively. (B, F) Larvae with Tc-six3 phenotype caused by pupal injection with a concentration of $100 \mathrm{ng} / \mu \mathrm{l}$. The median lobes of the MB are disarranged (white arrowheads, B and F). (C) pRNAi with 2,1 $\mu \mathrm{g} / \mu \mathrm{l}$, and (G) $1 \mu \mathrm{g} / \mu \mathrm{l}$. The MBs are severely affected and appear to touch each other (open arrow, G). (D, H) Larval phenotypes caused by pupal RNAi of $T c-c h x$ with a concentration of 1,9 $\mu \mathrm{g} / \mu 1 \mathrm{dsRNA}$. (D) The Central Body is not detectable. (H) The MBs are disarranged within the protocerebrum and the median lobes are more flexed (white arrowheads). Two putative pedunculi are present in the left hemisphere (open arrowheads).

altered. The median lobes were not located between the brain hemispheres like in wildtypes, but more laterally (white arrowhead, Figure 35, A). Furthermore, the Central Body was not detectable in these animals.

Increasing the concentration of injected dsRNA to $1 \mu \mathrm{g} / \mu \mathrm{l}$ for pupal RNAi (pRNAi) resulted in severe brain phenotypes. The brains appeared to be smaller than the WT brains and the posterior glial tissue was not unfolded (Figure 35, C). Nevertheless, the protocerebrum and the Antennal 
Lobes remained present (asterisks, Figure 35, C). The brain hemispheres were still separated, but the fissure between the hemispheres was strongly reduced. The Central Body and the median lobes of the Mushroom Bodies were not detectable (Figure 35, C). Further increase of dsRNA concentration to $2,1 \mu \mathrm{g} / \mu 1$ did not lead to any different or stronger phenotypes, neither on the brain nor on the cuticle level.

In order to test, whether Mushroom Bodies are deleted or absent, Tc-six3 pRNAi experiments were repeated with the same concentrations as described above in female pupae of the 'MB' line. Following injection of $100 \mathrm{ng} / \mu \mathrm{l}$ dsRNA of Tc-six3, all typical structures for Mushroom Bodies were found, i.e. Kenyon cells (KC) in the posterior glial tissue, Pedunculus (Pe), vertical lobes (vL), and median lobes ( $\mathrm{n}=6$; white arrowheads; Figure 35, F). The median lobes were not located centrally between the brain hemispheres, but lay bent within the protocerebrum (white arrowheads, Figure 35, F).

The brain phenotypes caused by injection of $1 \mu \mathrm{g} / \mu 1 T c$-six 3 dsRNA showed a strong reduction of the posterior glial tissue, whereas the Kenyon cells $(\mathrm{KC})$ and the Pedunculus $(\mathrm{Pe})$ were detectable $(n=5$; Figure 35, G). The latter partly touch each other at the midline, but appear not to be fused (open arrowhead; Figure 35, G). The vertical lobes and median lobes were not visible (Figure 35, G). Thus, the correct positioning of the Mushroom Body in the protocerebrum was affected by the weak Tc-six3 knock down. Furthermore, pupal knock downs of the gene Tc-six3 with higher concentrations caused severe defects in the brain as well. I.e. it affected the whole brain and also led to disarrangements of the Mushroom Body neuropile. Nevertheless, the most sensitive and most severely affected structure appears to be the Central Body. These results suggest, that Tc-six 3 plays an important role in the formation of the central brain and the Central Body of Tribolium castaneum.

Since $T c$-chx marks a subset of $T c$-six3 positive NBs, this gene displayed a very promising candidate for influencing the Central Body development. Knock down experiments yielded no cuticle phenotype, i.e. the larval cuticle had no defects and resembled the WT situation, independent of injections with $1,9 \mu \mathrm{g} / \mu \mathrm{l}$ or $3,8 \mu \mathrm{g} / \mu \mathrm{l}$ Tc-chx dsRNA. After Tc-chx RNAi in the 'Brainy' line, the resulting brain phenotypes appeared almost identical to those of the weak Tc-six3 RNAi phenotypes. The size of the brain hemispheres was the same as in WT situation, the Antennal Lobes were present and the posterior glial tissue showed no defects. Mushroom Bodies were present and only slightly malformed, i.e. the median lobes lay disarranged in the protocerebrum. The Central Body was not detectable (Figure 35, D).

Next to the 'Brainy' line, injections with the same concentration of $T c$-chx dsRNA were repeated in 
the 'MB' line to visualize possible effects on the Mushroom Body. Here, the Kenyon cells embedded by the posterior glial tissue were projecting to the calyx, from where the Pedunculus projected anterior ventrally into the protocerebrum, dividing into the vertical and the median lobe. The latter appeared to be slightly shortened and/or delocalized in the protocerebrum, while no abnormalities were detected for the position of the vertical lobe (Figure 35, H). However, in 50\% $(n=8 / 16)$ of the analyzed cases, malformed Mushroom Bodies were found. In these, the projections from the calyx were either split in two or more Pedunculi or even projections to the ventral lobes could be detected (open arrowheads; Figure 35; H). Furthermore, abnormal projections towards anterior arising, from the Kenyon cells and ending near the Antennal Lobes (not shown), were detected. However, such effects occurred in only one out of 13 Tc-six3 knock down animals. Interestingly, an increase of phenotype was not achieved by increasing the concentration of injected Tc-chx dsRNA.

Since no cuticle phenotype was detected, these analyses suggest, that $T c$-chx apparently exclusively influences the neural fate. Furthermore this gene was shown to play an important role in both Central Body formation and Mushroom Body development.

\subsubsection{Immunohistochemistry analysis of the brain phenotypes resulting from $T c$-six 3 and $T c$ - chx knock down}

Possibly occurring subtle defects may not be detectable by using the 'Brainy' and the 'MB' line. Therefore, the pRNAi phenotypes were characterized in immunohistochemic analyses. To this end, different antibodies against neuropeptides expressed in the larval brain and the Central Body were used. Female 'Brainy' pupas were injected with $100 \mathrm{ng} / \mu 1$ and $1 \mu \mathrm{g} / \mu 1$ Tc-six3 dsRNA to induce weak and strong phenotypes in the offspring. The brains of the larval offspring were dissected and stained with the respective antibodies (chapter 2.8).

Larvae with a weak RNAi impact showed separated brain hemispheres. Here, the anterior brain tissue surrounding the Antennal Lobes was separated like in the WT situation (weak RNAi phenotypes: Figure 36, B, F, K; WT situation: A, E, I). This was not the case in heavily affected RNAi phenotypes, where the median anterior tissue was fused (open arrows; Figure 36, C, G, L). Therefore, the brain was u-shaped here. Additionally, the whole brain appeared to be reduced in size

(Figure 36, C, G, L). These morphological characteristics were used to classify the observed phenotypes.

For the analysis of the Mushroom Bod, the antibody DcO was used (Zhao et al., 2008). The brains of weakly affected Tc-six3 pRNAi larvae had Pedunculi, vertical lobes, and median lobes ( $\mathrm{n}=10)$, 


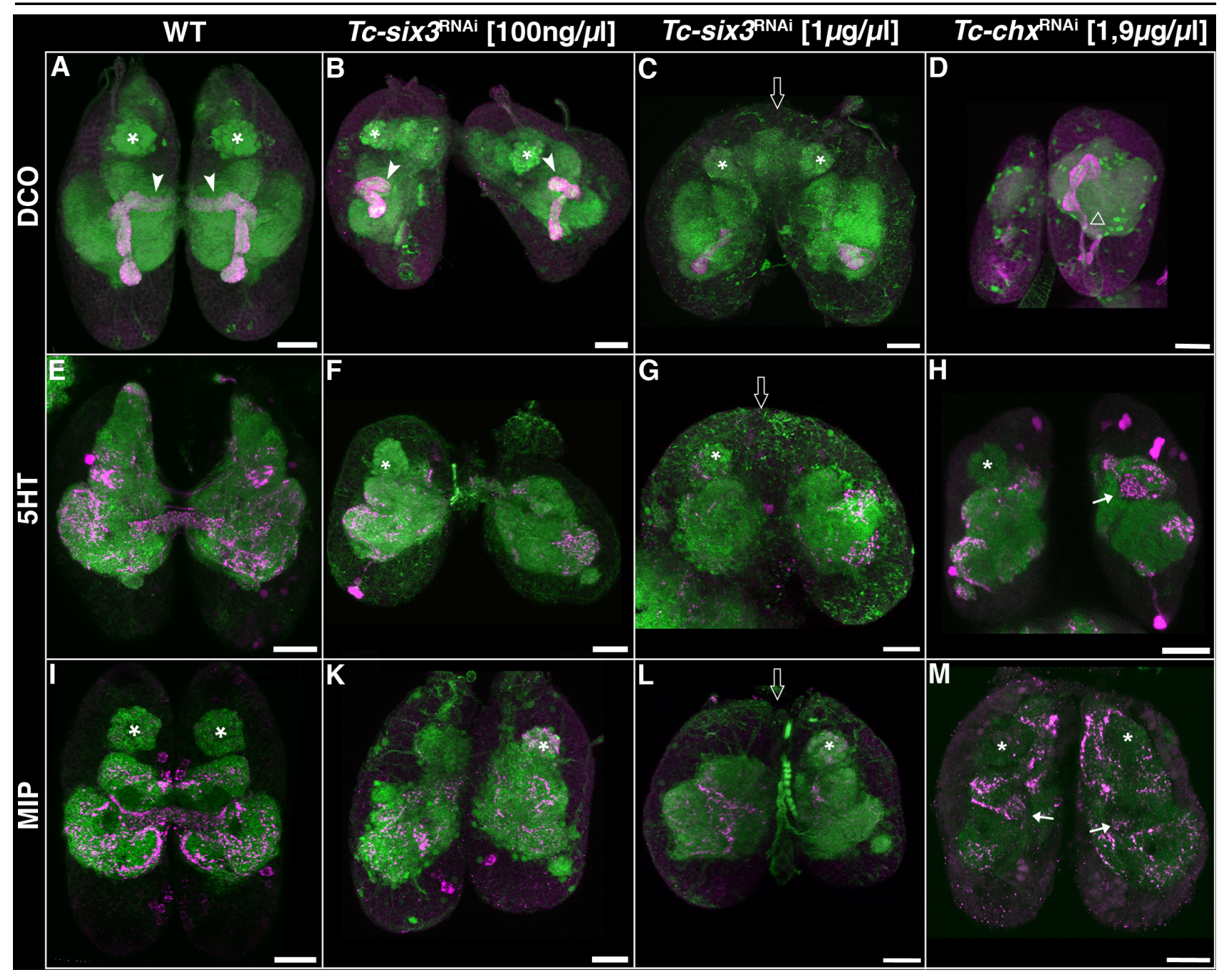

Figure 36: Tc-six3 and Tc-chx knock down leads to loss or cleavage of the Central Body (CB).

Immunohistochemistry stainings on larval brains. Larvae are offsrpring of Tc-six 3 and Tc-chx RNAi treated female 'Brainy' pupae. Depicted are dissected brains of first larval instars (L1). (A, E, I) Wildtyp (WT) L1-brains with antibody stainings for (A) DcO, (E) 5HT/ Serotonin, (I) Myo inhibitoric Peptide (MIP). (B, F, K) Tc-six3 pRNAi phenotypes caused by $100 \mathrm{ng} / \mu \mathrm{l}$ Tc-six3 dsRNA injections. (C, G, L) Tc-six3 pRNAi larval brain phenotypes caused by $1 \mu \mathrm{g} / \mu \mathrm{l}$ Tc-six3 dsRNA injections. (D, H, M) Tc-chx RNAi larval brain phenotypes caused by 1,9 $\mu \mathrm{g} / \mu \mathrm{l}$ dsRNA injections. All brains were costained with phalloiden, shown in green. In the first row (A-D) the antibody against DcO was used to stain the larval Mushroom Body (magenta staining). In the second row (E-H), embryos were stained with the antibody against 5HT/ Serotonin (magenta staining). In the third row (I-M), larval brains were incubated with an antibody against MIP. All brain phenotypes caused by Tc-six3 knock down show no CB. Phenotypes caused by Tc-chx knock down lead to a paired $\mathrm{CB}$ as indicated (white arrows; H, M), and further projections in the MB were detected (open arrowhed; D). Asterisks mark the larval Antennal Lobes. Anterior is facing up. All scale bars measure $20 \mu \mathrm{m}$. The preparation and staining of the WT and Tc-chx RNAi brains were performed by Martin Kollmann (University of Marburg) in collaboration.

even though these appeared to be reduced in size. The position within the protocerebrum was altered and especially the median lobes were heavily reduced (open arrowheads; Figure 36, B). The Antennal Lobes were present in all larvae. Strongly affected phenocopies of Tc-six 3 pRNAi, caused 
by injections of $1 \mu \mathrm{g} / \mu \mathrm{l}$ dsRNA, showed the Pedunculi of the Mushroom Body in all larvae (n=6), whereas the vertical and the median lobes were strongly reduced and the latter sometimes even not detectable at all (Figure 36, C). Generally, the Mushroom Bodies of these animals appeared reduced in size like the rest of the brain. Also in the strong phenotypes, the Antennal Lobes were present (asterisks; Figure 36, C).

The antibodies against Serotonin and MIP were used to mark the Central Body (Dacks et al., 2006; Predel et al., 2001). By performing stainings on Tc-six3 knock down brains, the absence of this neuropile could be confirmed in weakly affected larval RNAi brains. The neuropeptide serotonin was detected in the respective tissues of the brain $(n=12$, Figure $36, F)$, but a Central Body like structure could not be identified. In strong phenotypes this neuropile was also missing, while the neuropeptide was detectable in the rest of the brain $(n=12$, Figure 36, G). These results were confirmed by neuropeptide stainings for MIP, which marks the upper unit of the Central Body in adult animals (Martin Kollmann, personal communication; Predel et al, 2001). Here, an expression in the brain was detectable in both weak $(n=7$; Figure $36, K)$ and strong $(n=5$; Figure 36 , L) RNAi phenocopies of $T c$-six 3 . However, the median pattern was lacking, indicating a loss of median brain tissue including the Central Body.

Through these experiments it could be shown, that $T c$-six3 essentially contributes to proper development of the Tribolium castaneum brain. Importantly, the Central Body neuropile appeared to be most sensitive and did not develop after a knock down of Tc-six3. Furthermore, these experiments indicated a correlation of the injected dsRNA concentration and the strength of the phenotypes on cuticle as well as neural tissue level.

Immunohistochemistry analysis was also performed for the $T c-c h x$ RNAi larvae brain, similar to those described above. For this, Tc-chx pRNAi was performed by injecting female 'Brainy' pupae with a concentration of $1,9 \mu \mathrm{g} / \mu \mathrm{l}$ dsRNA. Brains of the respective offspring were dissected and stained using the antibodies described above.

Tc-chx RNAi $(1,9 \mu \mathrm{g} / \mu \mathrm{l})$ did not affect the separation of the brain hemispheres and rather mirrored the situation shown for weak Tc-six3 phenocopies. In Tc-chx phenocopies, no reduction of size and shape of the brain was detected, resembling the observations made in weak Tc-six3 RNAi phenotypes. The antibody against $\mathrm{DcO}$ was used to visualize the Mushroom Body. In Tc-chx phenocopies, structures like Pedunculi, median and vertical lobes were present. The median lobes were slightly shortened (not shown). Axon bundles positive for DcO staining were detected, arising from Kenyon cells projecting into the vertical lobes (open arrowhead; Figure 36, D). Therefore, the observations from the in vivo images could be confirmed in these stainings. 
To analyze the Central Body, antibodies against serotonin/5HT and MIP were used. The staining of knock down Tc-chx RNAi brains indicated that the Central Body was present but split. Anti 5HT staining was detected in the brain like in the WT situation. In addition, two out of four brains were found to have separated Central Bodies positive for anti 5HT (white arrow; Figure 36, H). The situation of the Central Body in the residual two brains could not be analyzed due to optical resolution. Similar results were obtained by the analysis of anti MIP stainings. All scanned brains showed separated Central Bodies ( $n=8$; white arrows; Figure 36, M). In two cases a Central Body was detected in only one hemisphere, while the other hemispheres could not be analyzed due to optical resolution.

$T c-c h x$ function is suggested to act exclusively on neural fate, since no cuticle phenotype was detected. Taken together, this gene plays an important role in the medial fusion of the Central Body and in the orientation of the developing Mushroom Body.

\subsubsection{Temporal separation of the epidermal and neural functions of $T c$-six 3}

Neurogenesis is a spatiotemporal process within the neuroectoderm (Urbach and Technau, 2003). In Tribolium, the first NBs delaminate at approximately $12 \mathrm{~h}$ at $32^{\circ} \mathrm{C}$ (chapter 3.3.1), while epidermal patterning starts much earlier. Now the question was, whether it is possible to separate the influences of Tc-six 3 in epidermic and neural fate by injecting embryos at different developmental stages. To address this, eggs of the 'Brainy' line were collected for $2 \mathrm{~h}$ and bred until the desired age at a temperature of $32^{\circ} \mathrm{C}$. Injections were performed with $T c$-six 3 dsRNA concentrated to $1 \mu \mathrm{g} /$ $\mu 1$.

Approximately one-third of the injected embryos survived the treatment and after breeding for 3-4 days on $32^{\circ} \mathrm{C}$ developed to larvae. The older the embryos were at the time of injection, the more embryos survived. Surviving larvae were randomly picked for preparation, scanning and analysis of the brain phenotype. This batch of larvae will further be called 'scanned larvae'. Afterwards, these larvae were embedded in Hoyers Lactic Acid to analyze the cuticle phenotype. Those embryos or larvae which were not selected, were used to prepare cuticles, in order to confirm that the picked embryos were showing a representative ratio of the respective phenotype. This batch will be called 'residual larvae'.

The earliest embryos were injected when developed $0-2 \mathrm{~h}$ hours at $32^{\circ} \mathrm{C}$ (Figure $37,0-2 \mathrm{~h}$ bar graphs). These embryos were in a very early blastodermal stage, where only a few energids exist. The 'scanned larvae' showed severe cuticle phenotypes. In 45,5\% (5 out of 11 analyzed cases), the anterior median tissue was deleted (compare Figure 34, C; Figure 37, E, 0-2h cuticle bar graphs). 


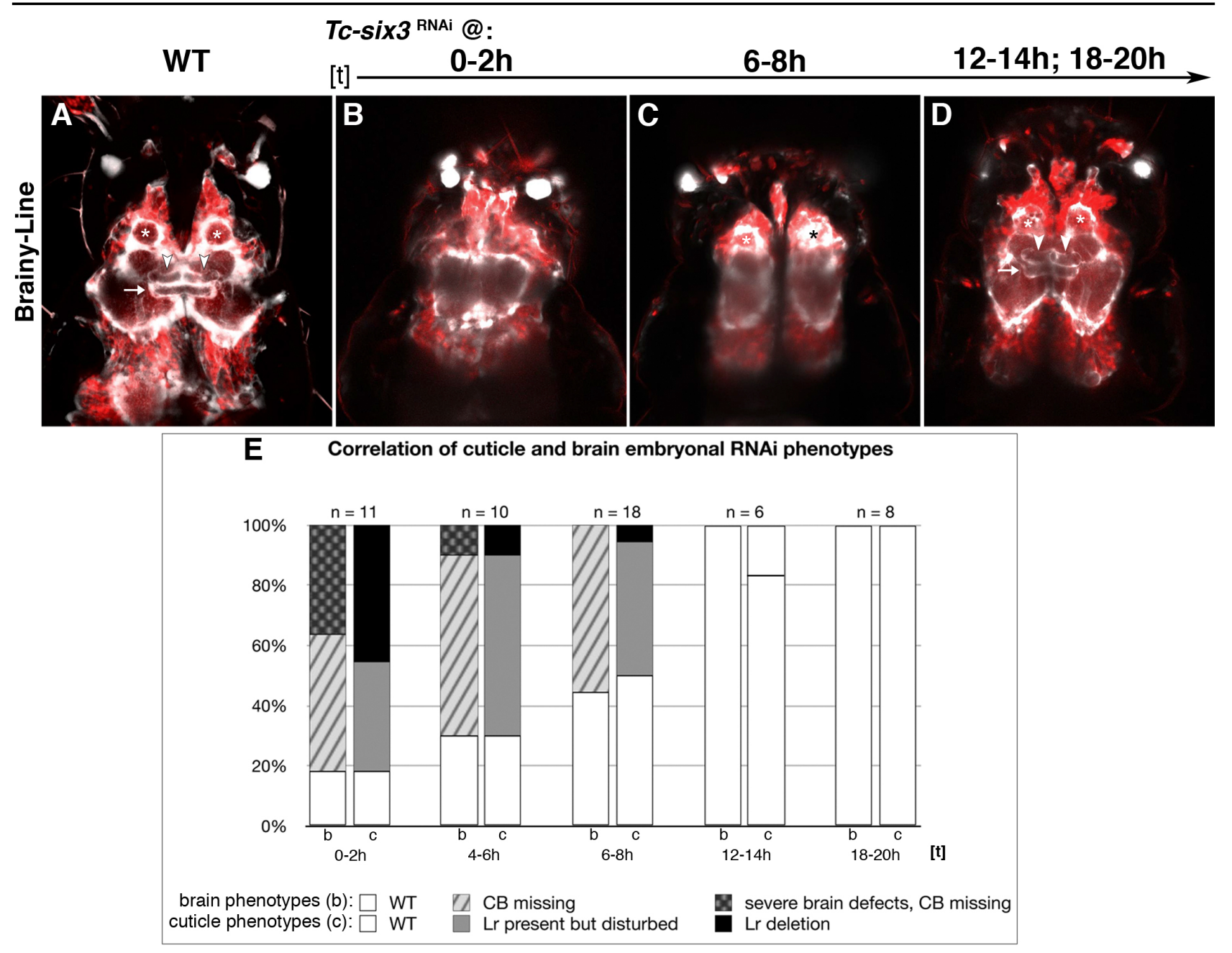

Figure 37: The epidermal and neural function of $T c$-six3 is not separable by late embryonic RNAi.

Embryos of the 'Brainy' (BA) line were injected with $1 \mu \mathrm{g} / \mu \mathrm{l}$ dsRNA of Tc-six3 at 0-2h, 4-6h, 6-8h, 12-14h, and 18-20h age. (A) WT situation in untreated BA line larva. (B) Strong brain phenotype induced by Tc-six 3 dsRNA injection at 0-2h. (C) Weak brain phenotype induced by Tc-six3 dsRNA injection at 6-8h, where the Central Body (CB) is not detectable and the brain hemispheres are separated. (D) Injections performed at 12-14h and 18-20h, where no defects were detectable. The larva depicted here originated from 18-20h injections series. The larvae are oriented to face anterior to the top. White arrowheads indicate the median lobes of the Mushroom Body. Asterisks indicate the Antennal Lobes, the arrow indicates the CB. (E) The graph depicts the distribution of larval brain and cuticle phenotypes caused by embryonic RNAi against Tc-six3 in percent depending on the time of injection. The left bars within each group reflect the distribution of larval brain phenotypes (b), while the right bar reflects the distribution of cuticle phenotypes in the respective embryos (c). The detached area in the cuticle bar [14-16h] represents a defect cuticle not reflecting the typical Tc-six3 RNAi phenotype. The defect was probably caused by an injection artefact. $\mathrm{n}=$ the number of individuals which were analyzed for brain and cuticle defects. 'Lr present but disturbed' represents the weak cuticle phenotype. 'Lr deletion' represents the strong cuticle phenotype.

This result correlated with the 'residual larvae', where $70 \%$ (7 out of 10$)$ showed a strong phenotype. Among the 'scanned larvae' the proportion of the weak cuticle phenotypes was 36,4\% (4 out of 11; compare Figure 34, B; Figure 37, E, 0-2h cuticle bar graphs), which correlated with $30 \%$ (3 out of 10 ) in the 'residual larvae'. No WT-like cuticle was detected among the 'residual 
larvae', while 18,2\% WT-like phenotype were detected among the 'scanned larvae' (2 out of 11; Figure 37, E, 0-2h cuticle bar graphs). Thus, in both cuticle analyses the strong phenotypes were dominant.

Following a knock down of Tc-six3 at later stages (4-6h), strong cuticle phenotypes were drastically reduced to $10 \%$ in the 'scanned larvae'(1 out of 10, Figure 37, E, 4-6h cuticle bar graphs). This also correlated with the analysis of the 'residual larvae', where the contribution of the strong phenotype decreased to $35,1 \%$ (13 out of 37). Larvae with weak cuticle phenotypes were represented with $60 \%$ among the 'scanned larvae' (6 out of 10; Figure 37, E, 4-6h cuticle bar graphs), while these represented 54,1\% (20 out of 37) in the 'residual larvae'. The portion of WT-like cuticles rose to $30 \%$ (3 out of 10; Figure 37, E, 4-6h cuticle bar graphs) among the 'scanned larvae' and to $10,8 \%$ (4 out of 37 ) in the 'residual larvae'.

In even later injections (6-8h), strong cuticle phenotypes were again drastically reduced to 5,6\% (1 out of 18; Figure 37, E, 6-8h cuticle bar graphs) in the 'scanned larvae' and within the 'residual larvae' no cuticle was found to show this phenotype. Even the batch of larvae with weak cuticle defects were decreased in both the 'scanned larvae' with $44,4 \%$ (8 out of 18; Figure 37, E, 6-8h cuticle bar graphs) and the 'residual larvae' with 30,9\% (17 out of 55). The portion of larvae without any phenotypes rose up to 50\% (n=9/18) among the 'scanned larvae' and to 69,1\% (38 out of 55) among the 'residual larvae'. Embryonic knock down of Tc-six3 at later stages than $12 \mathrm{~h}$ at $32^{\circ} \mathrm{C}$ age caused no detectable cuticle phenotype (Figure 37, E, 12-14h and 18-20h cuticle bar graphs). Thus, the severity of the Tc-six3 phenotypes decreased with the age of the embryos at the time of RNAi treatment.

For grouping the brain defects into strong or weak phenotypes, the same parameters as mentioned above were used. Strong phenotypes also had severe brain defects. Here, the median lobes of the Mushroom Body were not detectable, the posterior glial tissue appeared to be strongly reduced and the brain hemispheres were close to each other (Figure 37, B). Weak phenotypes showed malformed Mushroom Bodies and clearly separable brain hemispheres, but no Central Body (Figure 37, C). Strong brain malformations decreased from $36,4 \%$ at an injection age of $0-2 \mathrm{~h}$ ( 4 out of 11 ; Figure 37 , E, $0-2 \mathrm{~h}$ brain bar graphs), to $10 \%$ at an injection age of $4-6 \mathrm{~h}(\mathrm{n}=1 / 10$; Figure 37 , E, 4-6h brain bar graphs), and no strong phenotypes could be detected in injections in older embryos. Weak brain phenotypes were represented in $45,5 \%$ of the $0-2 \mathrm{~h}$ old RNAi treated embryos ( 5 out of 11 ; Figure 37 , E, $0-2 \mathrm{~h}$ brain bar graphs), in $60 \%$ of the $4-6 \mathrm{~h}$ old embryos ( 6 out of 10 ; Figure 37 , E, 4-6h brain bar graphs), and finally in $55,6 \%$ of the $6-8 \mathrm{~h}$ old embryos (10 out of 18 ; Figure 37 , E, $6-8 \mathrm{~h}$ brain bar graphs). 
A similar distribution of phenotypes became apparent when comparing brain and cuticle phenotypes (Figure 37, E). However, the brain tissue seems to be sensitive for RNAi with Tc-six3 for a longer time (Figure 37, E, 6-8h bar graphs). Apparently, there are two separate functions for Tc-six3. These can be divided into an early function leading, to strong phenotypes when performing RNAi, while a later function leads to impairment of the Central Body development. However, the Tc-six3 function essential for brain development cannot be separated from the epidermal function. Generally, Tc-six3 appears to be required for a proper brain development even at very early stages $(6-8 \mathrm{~h}$ still is an undifferentiated blastoderm). 


\section{Discussion}

\subsection{Reporter systems in Tribolium castaneum}

One part of this work was to establish transgenic reporter lines for visualization of the brain in Tribolium castaneum. The expression pattern of most of the lines that were generated differed from the expectations. This depends on many factors, e.g. the choice of the cloned regulatory regions, cloning and amplification artifacts, and also positional effects. In this paragraph, some features that could generally improve the reporter systems like the specific ones established here, will be discussed.

\subsubsection{Improving the Tc-repo and Tc-elav reporter lines}

In case of the 'reg.-Tc-repo::tGFP' reporter line, the expression pattern of the reporter gene appears to mirror that of the endogenous Tc-repo in early glia precursor cells. However, double in situ hybridization in 'reg.-Tc-repo::tGFP' embryos would further prove, if the mRNA expression patterns of Tc-repo and tGFP overlap exactly. In larval stages, only a weak fluorescent tGFP signal was detected. Both the delay in the tGFP mRNA expression pattern and the weak fluorescent signal in the larval stages indicated only a low expression level of the reporter gene. The resulting questions are, why the expression pattern is that light and how this could be improved?

One possibility to enhance tGFP expression, is to clone the total 5'UTR of Tc-repo. As described, half of the 5'UTR was not cloned due to the selection of optimal primers (chapter 3.2.2). The sequence of these primers was selected for a small number of hairpins and a high amount of GC bases, which was the case only $44 \mathrm{bp}$ upstream of the translation start site. This deletion could have resulted in a reduction the transcription of the reporter gene. This could be the reason that the levels of tGFP mRNA, which were detectable by whole mount in situ hybridization, were reached with a slight delay and that the fluorescent signal in the larval brain was only weak.

An alternative possibility to increase the reporter gene signal would be to duplicate the regulatory region. In many other reporter lines, a duplication of the regulatory region led to enhanced expression of the reporter gene. For example in the line $6 \times \mathrm{P} 3$ ::eCFP, the promoter sequence ' $3 \times \mathrm{PP}$ ' was cloned twice in tandem (Hein, 2007). In case of the 'reg.-Tc-repo::tGFP' line, the regulatory region has a length of $4510 \mathrm{bp}$. Therefore, an enhancer analysis would make sense in order to minimize the size of the sequence that needs to be duplicated. After duplication of this enhancer, the reporter gene signal in the embryonic stages could possibly be detectable earlier and closer resemble the endogenous Tc-repo expression. Furthermore, the signal in the larval brain would be enhanced, which would facilitate detailed colocalization analyses regarding the glial cells which are 
marked in the 'reg.-Tc-repo::tGFP' line. In any case, the expression of the reporter gene of the 'Tcrepo::tGFP' reporter line is congruent to the endogenous Tc-repo expression. This indicates that no positional effects of the insertion locus are present.

The reporter lines of 'Tc-elav::mRFP' showed an unexpected pattern in two lines derived from independent $\mathrm{G}_{0}$. Both showed an identical larval expression pattern in the muscles. An independent integration at the same genomic locus, resulting in the same enhancer trap, is very unlikely. This could only be proven by an identification of the respective integration locus of both transgenic lines. Interestingly, a search for orthologues of the gene Tc-elav revealed two genes in Drosophila, found in neurons (fne) and elav. A blast of the Drosophila genes within the Tribolium genome revealed in contrast only the single gene prediction Glean_4718, called Tc-elav (Simonnet, personal communication). Despite this unclear situation for the Tribolium homologues, the Drosophila transcripts and proteins of the genes Dm-fne and Dm-elav are restricted to neurons of the CNS during embryogenesis (Yannoni and White, 1997; Samson and Chalvet, 2003; Berger et al., 2007). In situ stainings for Tc-elav also showed an expression in the CNS during embryogenesis in Tribolium (Simonnet, personal communication).

One reason for the muscle expression pattern could be enhancer elements surrounding the gene locus of Tc-elav. The glean prediction Glean_4647, coding for a zinc carboxypeptidase, was identified in the upstream region of Tc-elav (chapter 3.2.1). This gene and its function are still unknown, so possibly it is expressed in muscles. Since access to alternative lines with specific CNS expression was possible ('MB' line and 'Brainy' line, chapter 3.4), the 'Tc-elav::mRFP' reporter line was not analyzed further.

\subsubsection{Low efficiency of the $T c-s i x 3$ reporter constructs}

The ability of promoters to be controlled by surrounding enhancer elements of the insertion locus was often proven and is used for large enhancer trap screens in various species (Klämbt and Goodman, 1991; Yang et al, 1995; Wurst et al., 1995; Horn et al., 2000; Balciunas et al., 2005; Trauner et al., 2009). This possibly explains, why the different lines carrying the same Tc-six3 reporter construct show different expression patterns. I.e. the offspring derived from two independent $\mathrm{G}_{0}$ carrying either the construct 'Tc-six3 10up' or 'Tc-six3 5up' did not show similar expression patterns. This suggests influences of surrounding enhancer and silencer elements of the insertion locus and low regulatory activity of the cloned fragments. To improve reporter lines, influences of surrounding enhancers could be avoided by either using insulator sequences or by site specific integration, for example by attP and attB integration (reviewed in Groth and Palos, 2004; 
Smith et al., 2010) which has recently become possible also for Tribolium castaneum (Kittelmann, personal communication).

Generally, the fluorescent reporter signal was very weak or absent in the lines carrying the constructs derived from $T c$-six3. The construct of the 'Tc-six3 promoter element' which was used as core promoter for all Tc-six3 constructs except for 'Tc-six3 5up', had a deletion of $9 \mathrm{bp}, 484 \mathrm{bp}$ upstream of the endogenous translation start site. This deletion, which occurred due to a PCR artifact, possibly caused a decrease in the transcription of the reporter gene tGFP. Thus, to exclude such a possible influence, new constructs which are including the entire core promotor could be created.

Another potential problem does not appear to play a role: The core promoter element of Tc-six3 included an additional ATG upstream of the reporter gene tGFP. These additional 18 bp are in frame, and cause additional 6 amino acids at the N-terminus of the tGFP protein. However, a fluorescent signal was succesfully detected for example in the line 7 carrying the reporter construct 'Tc-six3 10up'. Additionally, a fluorescent signal of tGFP was detected in vivo in the embryos of the reporter lines 6 and 3, which are carrying the construct 'Tc-six3 10up' and 'Tc-six3 5down' (data not shown). Therefore these constructs in principle express detectable tGFP levels.

\subsection{The 'Tc-rx 5up’ line for studies of axon guidance and brain midline cues}

The transgenic line 'Tc-rx 5up' shows a complex expression pattern of DsRedExpress in the larval brain. This appears to correspond to the endogenous $T c-r x$ expression (chapter 3.3.3.2). Therefore, this line is very promising for future studies of the development of the Central Complex and axon guidance in Tribolium.

The DsRedEx positive cell cluster is located in the posterior median part of the larval brain. This region correlates to the pars intercerebralis in insects, which is defined by its localization in between the brain hemispheres anterior mediolateral to the Mushroom Body and dorsally to the Central Body (white square, Figure 20; De Velasco et al., 2007; Steinmetz et al., 2010; Boyan and Williams, 2011; Boyan and Reichert, 2011). For Drosophila, it is known that the protein Chxl is expressed in the pars intercerebralis, while the homologue of $R x$ could be detected in the surrounding protocerebral tissue (De Velasco et al., 2007; Steinmetz et al., 2010). A definite verification and localization within the brain of the DsRedEx positive cells could possibly be performed by using antibodies specific for neuropeptides, which are produced by pars intercerebralis cells or specific for the $T c-R x$ protein. 
In Schistocerca and Drosophila, 4 NBs, which are playing an important role in formation of the Central Complex and which are located in the pars intercerebralis could be identified in the embryo and pupal brain. The neuronal progenies of such NBs are projecting anterior and crossing the brain midline. The orientation for axonal growth is given by an axonal scaffold forming commissures and connectives within the embryonic brain. At the onset of the Central Complex development, the commissures of the scaffold are already spanning the brain midline (Boyan and Williams, 1997; Williams et al, 2005; Boyan et al., 2008; Pereanu et al., 2011). In the transgenic line 'Tc-rx-5up' the DsRed positive cell cluster forms an axon bundle, which is projecting anterior and forms a chiasma by crossing the brain midline (white arrowhead, Figure 20). This axon bundle is located dorsally in the brain, but ventrally in relation to the neuraxis. Crossing experiments with the 6xP3 line revealed that this projection lies outside of the Central Body (chapter 3.3.3.2). Therefore, this axon bundle clearly does not belong to the Central Body and it thus did not derive of progenies of the 4 NBs known from Schsitocerca and Drosophila. However, the DsRedEx positive contralateral projections could belong to the commissures which form the scaffold and therefore give orientation to further axon growth. Immunohistochemistry stainings for horse radish peroxidase as a general neuronal marker (Jan and Jan, 1982; Kurosaka ate al., 1991), and for the reporter gene DsRedExpress should be performed in order to localize the respective axon bundle within the brain. It is known from several other species that the primary commissure pioneer (PCP) axons present the antigen Term-1, which is a glyocprotein secreted on the growth cones of neurons during axogenesis. At the same time, these axons are stainable with anti HRP (Meier et al., 1993; Ludwig et al., 2002). This is suggested to hold true for PCPs in Tribolium castaneum as well and colocalizaton should be tested with the respective antibodies in the 'Tc-rx 5up' line.

In this line, an additional DsRedEx positive structure could be detected anterior to the larval Central Body (chapter 3.3.3.2). By its shape and position within the brain, it possibly correlates to the Schistocerca lateral accessory lobes. These protocerebral functional units are tightly connected to the Central Complex (e.g. Boyan and Williams, 1997; Kurylas et al., 2008). In Schictocerca, a partial origin of the lateral accessory lobes lies in the pars intercerebralis (e.g. Boyan and Williams, 1997). However, in Tribolium, a connection between such anterior structure and the DsRedEx positive cells posterior in the brain surrounding the Kenyon cells is not detectable in the line 'Tc-rx

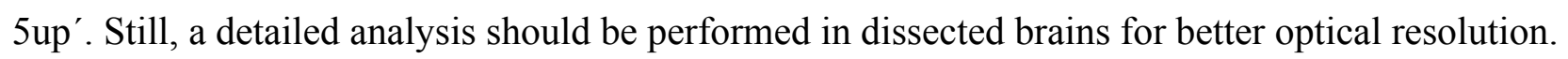

Taken together, the 'Tc-rx 5up' line marks an interesting axon bundle in the Tribolium castaneum brain as well as possibly the putative lateral accessory lobes (LAL). To address the embryonic axogenesis of the commissure and the putative LAL development, in vivo imaging could be 
performed, especially in combination with a $6 \times \mathrm{xP} 3:$ eCFP line. This would reveal, which of these parts arises first. If the mentioned axon bundle is a part of the scaffold surrounding and guiding the Central Body development, these axon bundles should be detectable before the Central Body is formed.

Furthermore, the 'Tc-rx 5up' line enables functional analyses regarding axon guidance. For example, a knock down of the genes Tc-robo and Tc-split could deliver insight into the ability of axons to cross the brain midline. The respective orthologue genes are known to act as receptor and ligand and to be involved in correct commissure formation in the CNS of vertebrates and invertebrates (Kidd et al., 1999; Brose et al., 1999; Jen et al., 2004; Dickson et al., 2006; Furrer et al., 2007; Coleman et al., 2010). Performing these functional analyses by using the 'Tc-rx 5up' line, defects on axon projection could be easily studied.

\subsection{The 'Brainy' reporter line allows analyses of embryonic brain development regarding the function of Tribolium homologues to vertebrate neural plate genes}

The 'Brainy' line is a crossing between two different lines that mark neuronal cells and glial cells (chapter 3.4). It is unknown, how much of the neuronal tissue is marked by the 'Brainy' line, and whether this line also marks neuronal cells derived from the mesectoderm. However, for the glial marking part (6xP3::eCFP) of this line, a coexpression analysis with an antibody for Repo was performed. From other species it is known that the repo homologue is expressed in all glial cells derived form the neuroectoderm, whereas glial cells derived from mesectoderm do not express this gene (Yuasa et al., 2003; Lee \& Jones 2005; reviewed in Parker et al., 2006). The coexpression analyses of the 6xP3::eCFP and the anti Repo signal in Tribolium were performed in colaboration with Martin Kollmann and Joachim Schachtner (University of Marburg). They revealed that 98,8 \% of the eCFP positive cells at the same time also express the protein Repo, whereas $84,2 \%$ of the Repo positive cells are also positive for the eCFP signal (Kollmann et al., unpublished). This suggests, that the eCFP positive cells of the 'Brainy' line are glial cells, but that not all glial tissue is marked by this line, as approximately $15 \%$ of the Repo positive glial cells are not detectable in the 'Brainy' line.

Although not all cells may be marked, this reporter line enables visualization of embryonic brain development in Tribolium castaneum (chapter 3.1.4). Surface glia marked by the expression of eCFP enable visualization of the borders of most neuropiles like the Antennal Lobes, the Central Body and the Mushroom Body in the larval brain. Nevertheless, the neuropiles themselves are not detectable in this line. Also the paired Protocerebral Bridge in the posterior part next to the calyces 
(chapter 1.5), appears not to be enveloped by glial cells yet. Additionally, in the Tc-chx RNAi phenotypes, the split Central Body was not detectable by in vivo imaging but required antibody staining. This was probably due to the lacking glial surface. These two examples demonstrate the limitations of this line in investigating gene functions in neuropilear organization. Despite these limitations however, the 'Brainy' line offers the opportunity to investigate the overall larval brain architecture. It offers the first step in the identification of genes, which have a strong influence on neural architecture and/or glial tissue. Thus, the 'Brainy' line established in this work is a very promising tool for first gene function analyses regarding embryonic brain development in Tribolium castaneum.

In Schistocerca, it is known that the protocerebral commissure develops when $31 \%$ of embryonic development is completed and when the protocerebral lobes lie next to each other. By dissolving of the glial bound borders, primary commissure pioneer axons derived from the mesectoderm cross the brain midline and serve as a scaffold for further midline crossing axons (Boyan et al., 1995; Ludwig et al., 2002). In the case of Tribolium castaneum, the development of the protocerebral commissure has not been studied yet. Here, the 'Brainy' line gives first clues to the point in time of its development. After a development of $30 \mathrm{~h}$ at $32^{\circ} \mathrm{C}(35 \%)$, the protocerebral lobes of the embryo become detectable and then grow to strong branches in up to $40 \mathrm{~h}(48 \%)$ old embryos. The hemispheres lie close to each other, separated by a border consisting of glial cells. In $45 \mathrm{~h}(53 \%)$ old embryos, the commissure is already visible and could also be confirmed by using the DIC-filter in case of Tc-chx in situ hybridizations in an embryo aged 40h - 55h (chapter 4.3.1). This indicates, that in the time slot of $30 \mathrm{~h}$ to $40 \mathrm{~h}$ which correlates to approximately $35 \%$ to $48 \%$ of the embryonic development, the primary commissural pioneers axon should have passed the brain midline. Apparently, the timing is very conserved between the mentioned insects. A more detailed analysis of axon scaffold and commissure formation would be possible by anti HRP and anti-Term1 stainings.

Various aspects could be addressed in future studies using this line. How are the brain midline features established, i.e. are genes like netrin, roundabout, slit, and Dscam also involved in midline passing controls in the brain of Tribolium castaneum (reviewed in Evans and Bashaw, 2002; Edwards and Meinertzhagen, 2010)? Which genes expressed in the neuroectoderm influence the development of neuropiles or parts of the brain? For example, what is the role of the identified 18 genes in Tribolium castaneum which are homologues to genes expressed in the vertebrate neural plate genes and which are also expressed in the head during embryonic stages (Posnien, 2009)? Until now, only effects on the epidermis could be studied by RNAi technique in Tribolium 
castaneum, but using the 'Brainy' line now also neurogenic aspects can also be addressed. This will be especially interesting for the genes, which showed only minor defects on the larval head cuticle after the respective knock down, e.g. Tc-ey, Tc-toy, Tc-so/sixl, Tc-dbx, Tc-ptx/pitx, Tc-mirr/irx, Tcmun/arx, Tc-fez, Tc-gsc, Tc-tll and Tc-Dll (Posnien, 2009). The 'Brainy' line allows the rapid identification of genes with strong effects on brain morphology upon RNAi. This would be especially interesting for the embryonic Central Body development in Tribolium castaneum, because Drosophila melanogaster does not develop this structure in the embryo.

\subsection{Results regarding the embryonic brain formation in Tribolium castaneum}

In this work, various genes were analyzed regarding their function in embryonic brain development in Tribolium castaneum. The major focus lay on those genes, which had a putative influence on the Central Body formation, since Drosophila lacks such a neuropile in larval stages. The different aspects and gene functions according their loss-of-function phenotypes will be discussed and finally summarized in a model.

\subsubsection{Tc-otd1 has a conserved function in specifying the anterior part of the brain}

In previous analyses, a knock down of Tc-six3 by parental RNAi led to the expansion of the Tc-otd1 domain in the neuroectoderm. Vice versa, a repressing function for Tc-otdl on the expression of Tcsix3 was hypothesized, forming a negative feedback loop to retain the boundaries of the expression domains (Posnien, 2009). During embryonic development, two major functions were suggested to be carried out by Tc-otd1: First of all in axis formation and regionalizing of blastodermal fate during the early blastodermal stages and secondly in head patterning in embryonic later stages (Schinko et al., 2008; Kottkamp et al., 2010).

To gain insight into the Tc-otdl function on brain patterning, embryonic knock down was performed in different stages in this work (chapter 4.2). Here, in weak phenotypes a reduction of the posterior glial tissue was observed, whereas in strong phenotypes the entire brain architecture was affected (chapter 4.2). Intermediate phenotypes, which reflect the transition from weak to strong effects were not detected. Thus, a true intermediate phenotype as it was expected to be caused by time dependent embryonic injections was missing.

In previous studies, embryonic Tc-otdl RNAi experiments were performed and revealed cuticle based phenotypical series. The various outcomes of time dependent injections suggested different functions in development: Once a regionalizing function in blastodermal stages and secondly a head patterning function in embryonic later stages (Schinko, et al., 2008). In the case of head patterning, 
Tc-otdl function has to take place in embryonic stages where the head anlage is already existing. Hence it influences the ectodermal as well as the neural fate. Interestingly, in this work, the cuticle phenotype and the brain phenotype correlate with each other. Whenever a strong cuticle phenotype occurred, the brain was strongly affected as well. The same correlation was observed in weakly affected RNAi embryos. Focusing on the brain phenotypes, in embryos injected at an age of 7-8h old at $32^{\circ} \mathrm{C}$, the strong phenotypes were dominant while only weak phenotypes occurred in embryos injected later (chapter 4.2). This indicates a temporal specification and patterning function of Tc-otdl in the anterior brain. The obtained results led to the question, when this stage of transition between strong and weak phenotype occurs? Apparently, this time is between $7-8 \mathrm{~h}$ at $32^{\circ} \mathrm{C}$ when the embryos are still in a blastodermal stage and Tc-otdl is already strongly expressed (Schröder, 2003; Schinko etal., 2008; Kotkamp et al., 2010). Therefore, a knock down of Tc-otd1 mRNA does not immediately affect the Tc-Otd1 protein level which is dependent of the protein turn over. Antibody stainings for Tc-Otdl could reveal how fast levels of this protein are reduced after RNAi. Thereby, it would be possible to better identify the transition between the strong and the weak phenotype.

The function of otd homologues in regionalizing the anterior part of the brain was suggested to be conserved for invertebrates and vertebrates (Hirth and Reichert, 1999; Lichtneckert and Hirth, 2005; Lichtneckert and Reichert, 2008; Hirth, 2010). In Drosophila, a loss of otd results in the loss of the entire anterior brain, probably due to the absence of most protocerebral and partially also the deuterocerebral neuroblasts in such mutants (Hirth et al., 1995; Younossi-Hartenstein et al., 1997). In mouse, Otx2 null mutants the entire forebrain and midbrain are absent (Acampora et al., 1995). Therefore, the strong phenotypes in Tc-otdl RNAi probably reflect an anterior brain deletion. However, in some scans of strong Tc-otdl knock down brain phenotypes, structures which possibly are parts of the Mushroom Bodies could be detected (not shown). To verify this situation, Tc-otdl knock down experiments together with immunohistochemic analyses using the Mushroom Body marker DcO could be performed. In Drosophila, the NBs which form the Mushroom Body neuropile arise in the protocerebrum (Noveen et al., 2000). Studies of correlating expression patterns of homologue genes like dachshund and eyeless also identified a correlating region in the protocerebrum that gives rise to Mushroom Body NBs in Tribolium (Hein, 2007; diploma thesis). This suggests, that the Mushroom Bodies are protocerebral structures and therefore part of the anterior brain. Therefore, if the hypothesis of anterior brain deletion holds true, the Mushroom Body should not be detectable after a knock down of Tc-otdl (Acampora et al., 1995; Hirth et al., 1995; Younossi-Hartenstein et al., 1997). 
In an alternative hypothesis, Tc-otdl is required for cell survival and therefore a knock down leads to a reduction of the brain. Even though mostly unknown, one of the putative target genes of $T_{c}$ Otdl during blastodermal stages was suggested to be a housekeeping gene (Kotkamp et al., 2010). Thus, a disturbance induced by knock down of Tc-otd1 could lead either to a loss of cell fate in the developing brain, followed by programmed cell death, or to the loss of the housekeeping gene, which in turn also leads to cell death. Both situations could explain the drastically reduced brains with only partially present neuropiles. To identify, whether a gap like deletion occurs after a knock down of $T c$-otd1, specific antibody stainings against proteins like $T c-S i x 3, T c-R x, T c-C h x$ or DcO expressed in the protocerebrum could be performed. If these proteins are still detectable, a complete deletion of the anterior brain can be excluded. To investigate the possibility of cell death, a TUNEL (terminal deoxyribonucleotidyl transferase [TDT]-mediated dUTP-digoxigenin nick end labeling) assay of the larval RNAi brain could be performed (Gavrieli et al., 1992; Prpic and Damen, 2005). By this it could be estimated, if a knock down of Tc-otdl leads to an increase in cell death in the developing embryonic brain.

Nevertheless, the function of the anterior regionalization of the anterior brain is conserved among the species (reviewed in Lichteneckert and Reichert, 2005). Therefore it is likely, that the knock down phenotype of Tc-otdl in Tribolium mirrored this conserved function and lead to the deletion of the anterior parts of the brain. For this function, Tc-otdl appears to be required rather early in development. Alternative options for this drastical brain phenotype would be that genes involved in cell fate specification require $T$ c-otd 1 input, or that the expression of a housekeeping gene could be affected after a knock down of Tc-otdl in embryonic stages (Kotkamp et al., 2010).

\subsubsection{The role of $T c-r x$ in Central Body formation}

In vertebrates, $R x$ homologues play an important role in eye and brain development. An overexpression can induce ectopic retinal pigmented epithelium, duplications of the retina and the neural tube (Chuang and Raymond, 2001; Bailey at al., 2004). In contrast, the Drosophila homologue $d r x$ was found not to be required for establishing the visual system (Davis et al, 2003) and it was not even found to be expressed in embryonic and larval eye primordia (Eggert et al., 1998). Tc-rx is not expressed within the developing eye field (Posnien, 2009; chapter 3.3.1). This seems to be confirmed by Tc-rx RNAi phenotypes, where no detectable malformation was observed in the larval eyes. This aspect is however preliminary and has to be explicitly studied by immunohistochemistry. However, $r x$ homologues of insects and other invertebrates appear not to have functions in eye development (Arendt et al., 2002; Davis et al., 2003; Arendt et al., 2004). 
Yet, Tc-rx RNAi leads to a split Central Body which is located at the midline in the larval brain. The split parts are surrounded by glial cells and indicate a loss in midline passing signals (chapter 4.1). $T c-r x$ expression starts at the point in time when 5-8 wingless stripes occur, which can be correlated to an approximate age of $12-15 \mathrm{~h}$ at $32^{\circ} \mathrm{C}$. At the onset of expression, it is bilaterally expressed at the anterior median rim of the head lobes. In later stages, the expression pattern shifts to a more lateral position at the rim of the head lobes. After head morphogenesis, the Tc-rx expression domains are positioned in the middle of the head laterally to the midline (Posnien, 2009; chapter 3.3.1). A quantification of the amount of NBs which arise from $T c-r x$ positive regions revealed 11 up to an age of $18 \mathrm{~h}$ at $32^{\circ} \mathrm{C}$ (see chapter 3.3.1). Furthermore it is suggested by the double stainings, that the $T c-r x$ positive cells in the brain may derive from such NBs.

The role of $T c-r x$ in formation of the neuroendocrine system was suggested to be conserved among bilaterians. Homologues of $r x$ were discussed to be involved in forming the neuroendocrine system in Drosophila, Platynereis, zebrafish and other animals (Hartenstein, 2006; de Velasco et al., 2007; Tessmar-Raible et al, 2007). Although the specific role of $d r x$-positive neurons in the pars lateralis of Drosophila are still obscure, they possible form extra neurosecretory cells which are not connected with the ring glands (de Velasco et al., 2007). Steinmetz and colleagues (2010) assume that the neurosecretory system in bilaterians derives from cells in the anterior part of the neuroectoderm/brain precursor which are six 3 positive. The Central Body forming neuroblasts were identified to be located in the pars intercerebralis in the embryonic brain of Schistocera, as well as in the pupal brain of Drosophila (reviewed in Boyan and Reichert, 2011). There is no obvious connection between $r x$ positive regions and these NBs which contribute the Central Complex. Interestingly, in $d r x$ mutant Drosophila flies, the development of the ellipsoid body which is part of the Central Complex and the homologue structure to the lower unit in other insects, is malformed or even unfused (Davis et al., 2003). Davis and colleagues (2003) discussed a mechanism where neurons in $d r x$ mutant flies either change the ability of their growth cones to respond to midline cues or that pioneer tracts or glia for proper ellipsoid body placement and formation are missing. In the larval head of Tribolium, only the lower part of the upper unit of the Central Body exists (chapter 1.5). However, a knock down of Tc-rx leads to an unfused Central Body, similar to that observed in Drosophila mutants. Despite the different stages, $r x$ appears to play a similar role in embryonic and larval Central Body formation in Tribolium and Drosophila, respectively.

Eventually, the question how a split Central Body can arise, remains. In Drosophila, genes, which play important roles in axon pathfinding and especially in midline crossing, could be identified (reviewed in Evans and Bashaw, 2010). The receptor proteins Round about (Robo) have been 
analyzed with respect to their contralateral axon guidance ability in the Drosophila brain. Here, a loss-of-function study in Robo2 and Robo3 mutants discovered malformations of the Central Complex, especially the fan-shaped body and the ellipsoid body. The axons which are crossing the brain midline in the WT-situation, are concentrated at the brain midline here but do not cross (Nicholas and Preat, 2005). When comparing this to the Tribolium Tc-rx knock down phenotype, certain similarities appear, as this leads to a split Central Body (chapter 4.1). Similar to the Drosophila Robo mutants, the split parts of the Central Body are at the correct positions within the protocerebrum in Tribolium. Additionally, the split compartments are enclosed by glial cells like in the WT-situation in Tribolium. The presence of surface glia in Tc-rx knock down phenotypes makes a specific glial function of $T c-r x$ unlikely, but nevertheless it cannot be excluded. In contrast, the described split Central Body phenotype in Tc-rx knock down Tribolium larvae suggests a lack of cues for midline crossing. Possibly the growth cones of the processing Central Body neurons are affected, which would lead to a loss of their capability to cross the brain midline. Also extrinsic cues, for example the proper formation of a primary axon scaffold, or even the presence of ligands or other factors, which influence the axon guidance at the brain midline, could be missing. From the expression pattern of Tc-rx in embryonic stages (chapter 3.3.1 and 3.3.3.2) and from the 'Tc-rx 5up' reporter line (chapter 3.3.3.2) no evidence that $T c-r x$ could be expressed in Central Body forming neurons is provided. It is more likely that $T c-r x$ is required for formation of the axon scaffold which is necessary for the midline crossing of the Central Body axons.

To prove the axon scaffold building hypothesis, an additional Tc-rx RNAi experiment could be performed in the 'Tc-rx 5up' reporter line. An absence of such a scaffold would lead to a general breakdown of the midline spanning projections in larval stages, meaning the DsRedEx positive axon bundles would not cross the brain midline. An anti HRP staining in Tc-rx RNAi animals would confirm this and address this question in an embryonic stage. Also, antibodies specific for Tc-rx would offer the possibility to identify putative targets of $T c-r x$ by chromatin immunoprecipitation (e.g. Orlando, 2000). The findings from such analyses would possibly help to elucidate the function of Tc-rx concerning the cell specification and formation of the Central Body in the embryonic and larval brain of Tribolium castaneum.

\subsection{3. $T c-s i x 3$ and $T c-c h x$ are required for Central Body formation}

The results of this work showed that Tc-six 3 is important for the proper development of the larval Central Body during embryogenesis in Tribolium castaneum. What role does Tc-six3 play during the embryonal development and the development of the Central Body? Taken together, Tc-six 3 is 
exclusively expressed in the head during later stages, i.e. in the anterior most part of the embryo tissue, the labrum and surrounding tissue. Within the Tc-six 3 positive tissue, at least 8 NBs arise. RNAi experiments revealed that in strong phenotypes the median head epidermal as well neural tissue is deleted, while in weak phenotypes mainly the brain is affected through the loss of the Central Body.

Due to these results, two functional phases for Tc-six 3 during embryogenesis are suggested, once a proliferative and second a neural fate specifying function. The strong phenotype suggests a proliferative function of Tc-six 3 and is characterized by the loss of the median head including the median brain. Especially the immunohistochemistry experiment revealed the absence of MIP positive cells in the median brain region after a knock down of Tc-six3 (chapter 4.3.4). The absence of these cells in knock down larval brains indicates their origin in tissue, which depends on Tc-six 3 function. In vertebrates, a cell proliferative function for SIX3 was detected and a misfunction of SIX3 leads to apoptosis in the anterior neural ectoderm and finally to holoprosencephaly (e.g. Loosli et al., 1999; Gestri et al., 2005; Geng et al., 2008). In mice, mutants with hypomorphic alleles of SIX3 and SHH revealed higher apoptosis rates in distinct regions of the lateral dorsal telencephalon, which was suggested to cause a microcephaly phenotype and in addition showed a slight reduction of proliferating cells (Geng et al., 2008). Due to this, Tc-six3 is suggested to play an important role in cell proliferation in the anterior median tissue. To test this, apoptosis and proliferation changes should be quantified by TUNEL (Gavrieli et al., 1992) assay and phosphohiston-3 staining (Hendzel et al., 1997). An enhanced cell death and/or a reduced proliferation in Tc-six3 RNAi could explain the loss of the anterior neuroectoderm, from which cells positive for MIP and serotonin and cells required for Central Body formation normally arise.

While the strong phenotype indicates an early function of $T c$-six 3 in cell proliferation and therefore in forming the correct amount of cells, the weak phenotype indicates a more specific neural determining function. Weak phenotypes revealed a total loss of the Central Body, while the cuticle was weakly or not at all affected (chapter 4.3.3, 4.3.4, and 4.3.5). Neither in RNAi in vivo scans nor in the respective immunohistochemistry stainings, were this neuropile or parts of it detectable, while the remaining brain appeared to be physiologically and morphologically unaffected (chapter 4.3.3 and 4.3.4). Due to the expression pattern of Tc-six3, which overlaps with the pars intercerebralis marker Tc-chx (chapter 4.3.1; de Velasco et al., 2007; Steinmetz et al., 2010), it is suggested that the pars intercerebralis tissue is affected by a knock down of this gene. From Schistocerca it is known that some NBs of the pars intercerebralis contribute to the Central Complex and to neurosecretoric cells, and to the primary commissure pioneer cells, which are 
expressing Term-1 and are positive for anti HRP ((Boyan and Williams, 1997; Williams et al., 2005; Williams et al., 2008; Boyan et al., 2010, Herbert et al., 2010; Ludwig et al., 2002). Thus it is suggested that a knock down of Tc-six3 leads to the loss of large portions or even to the loss of the entire pars intercerebralis in Tribolium. Taking into account that the primary commissure pioneers arise in the pars intercerebralis of Tribolium as well, no commissures would be detectable in knock down brains. This could be tested by respective antibody stainings, e.g. anti HRP.

Most evidence for such separable functions of Tc-six3 during the embryonic development is delivered by the embryonic knock down experiment (chapter 4.3.5), where RNAi was performed at various times of embryonic development. The early knock down of Tc-six3 reveals strong phenotypes and indicates the proliferative function of Tc-six3, as described above. I.e. the knock down of Tc-six 3 at early stages finally leads to the loss of the accurate cell number, hence to the loss of the median tissue, epidermally as well as neurally. However, a knock down of Tc-six3 at later stages, revealed the weak phenotype (see above), which much more represents a neural function. Here, Tc-six 3 seems to be required to specify neural cells, which form the Central Body (chapter 4.3.5). Thus, the function of Tc-six 3 seems to switch from an early proliferative to a late neural specifying function during the embryonic development in Tribolium castaneum.

Intriguingly, these functions appears to be dose dependent. Pupal knock down experiments with differing Tc-six3 dsRNA concentrations $(1 \mu \mathrm{g} / \mu \mathrm{l}$ and $100 \mathrm{ng} / \mu \mathrm{l})$ revealed that injections of low concentrations cause mainly Central Body defects, thereby reflecting the weak phenotype, while injection of highly concentrated Tc-six 3 dsRNA lead to a additional loss of median brain marking MIP expression, thereby reflecting the strong phenotype (chapter 4.3.4). In medaka, loss and gain of function experiments of Six 3 revealed a dose dependency in proliferation and differentiation in retinogenesis (Del Bene et al., 2004; Singh and Tsonis, 2010). High levels of Six3 allowed proliferation and inhibited both the antagonist Germinin and premature neuronal differentiation (Del Bene et al., 2004; Singh and Tsonis, 2010). In correlation to pupal RNAi in Tribolium, the injection of a higher concentration of Tc-six 3 dsRNA appears to lead to the loss of the regarding threshold to enable cell proliferation at all, i.e. those cells either acquire a different fate, undergo cell death or start differentiation. Nevertheless, the anterior median region is lost. In contrast, the phenotypes caused by injections of lower concentrations of dsRNA could be explained by either a partial loss of cells, due to a reduced proliferative phase, or by premature differentiation, which results in the situation of too little cell numbers, at the end. Furthermore, it became clear by these experiments, that the first and early function of cell proliferation is highly regulative, while the second and later function of neural cell specification is not at all or not strongly regulative. I.e. to 
achieve that the cell proliferative function of $T c$-six 3 is interrupted, high doses of $T c$-six 3 dsRNA are needed, while injections of lower dsRNA doses are still affecting the neural cell specification, especially the Central Body progenitor cells. Thus, Tc-six3 function in embryonic development has two phases which are additionally dose dependent.

The function of six 3 homologues have been discussed to be conserved among the bilaterians. In mice it is expressed in the most anterior region of the neural plate, from which neural derivates like hypothalamus, optic vesicle, ventral forebrain and neurohypohysis derive (Oliver et al., 1995). Steinmetz and colleagues (2010) showed that six3 homologues are expressed in the anterior tip during embryonic stages in Platynereis, Pristina, Euperipatoides, Strigamia, and Tribolium and thereby show a conserved expression pattern among bilaterians. Furthermore the authors showed that the Drosophila Six 3 homologue protein is detectable in a large region in the embryonic brain, which gives rise to the neuroendocrine system. However, the expression domain overlaps with cells of the pars intercerebralis, which are marked by the expression of Drosophila chxl (Steinmetz et al., 2010). In Schistocerca, a lot is known about neuroarchitecture and its development of the Central Complex during embryogenesis (Boyan and Williams, 1997; Williams et al., 2005; Williams et al., 2008; Boyan et al., 2010, Herbert et al., 2010). Here, eight bilateral NBs are located at the brain midline in the pars intercerebralis and contribute to the formation of the embryonic Central Body. Four per hemisphere play the key role in Central Body development and their progenies establish the basic columnar morphology of this neuropile (Boyan and Reichert, 2011). In Drosophila, recent studies identified 4 NBs to be involved in establishing the basic morphology of the Central Complex during late larval stages and the puparium (Itzergina et al., 2009). The expression pattern of Tc-six3 had already been shown to be conserved (Steinmetz et al., 2010; chapter 3.3.1). Additionally, the respective $T c$-six 3 positive region correlates to the location of the arising NBs, which form the Central Body in Schistocerca and in Drosophila. Tc-six3 plays an important role in specifying the NBs to form the Central Body, which is demonstrated by the fact that the Central Body is lost after Tc-six3 RNAi in Tribolium larvae (chapter 4.3.3). Thus in Tribolium, the Central Body forming NBs arise in the Tc-six3 positive region (chapter 3.3.1). Therefore, the suggested role for Tc-six3 in establishing parts of the neuroendocrine system in Tribolium could be confirmed by this work. The first indication was delivered by the overlapping expression pattern with the pars intercerebralis marker $T c$-chx, but a clear evidence is the loss of the expression of the neurotransmitter serotonin and the neuropeptide MIP in the central brain after Tc-six3 RNAi (chapter 4.3.4).

Due to the facts, that in Drosophila chxl was identified to specifically mark the pars intercerebralis 
(de Velasco et al., 2007) and the correlation of NBs derived from the pars intercerebralis forming the Central Complex in Schistocerca (Boyan and Williams, 1997), the Tc-chx orthologue was an interesting candidate gene for the elucidation of the NBs in Tribolium. Additionally, it was identified to be overlapping in its expression with the gene Tc-six3 in Tribolium (chapter 4.3.1) and this was also observed for the orthologue genes in Drosophila (Steinmetz et al., 2010). This combination of observations lead to the hypothesis that Tc-chx could specify the neural lineage responsible for the pars intercerebralis and NBs, which form the Central Body in Tribolium. After RNAi for Tc-chx in the 'Brainy' line, no structure ensheathed by glia resembling the Central Body in the larval brain could be detected. However, in immunohistochemistry analyses, structures positive for MIP and serotonin located laterally in the larval brain were detected. These findings support the hypothesis of displaying remnants of the Central Body (chapter 4.3.4). However, what could be the reason for the 'split Central Body phenotype', which is positive for MIP and serotonin staining, after a knock down of $T c-c h x$ ? Are these lateral structures really Central Body remnants?

The position of the MIP and serotonin positive structures do not support the theory of a split Central Body. In WT, the Central Body is located in the center of the brain, while in Tc-chx RNAi embryos, the position of the MIP and serotonin positive structures is lateral within the protocerebrum and they appear to be shifted to a ventral position. In comparison to the Tc-rx phenotype, which shows a split Central Body (chapter 4.1), the positioning of such remnants was WT-like. Additionally, the remaining parts of the Central Body were surrounded by glia cells in Tc-rx RNAi phenotypes, which was not the case in $T c-c h x$ RNAi. Also, since the regarding glial cells are missing after $T c$ chx RNAi, it is suggested that those derive from the $T c-c h x$ lineage.

In Tenebrio molitor, immunohistochemistry analyses for serotonin positive neurons revealed that such neurons innervate the fan-shaped body in later larval stages (Wegerhoff and Breidbach, 1992). I.e. serotonergic neurons enter an existing Central Complex. Transferred to the situation in Tribolium, the Central Body is possibly absent after a knock down of Tc-chx, but that MIP positive and serotonergic neurons innervate any structure in the protocerebrum, but not the Central Body. This 'non Central Body structure' could be what was finally detected in immunohistochemistry analyses of $T c-c h x$ knock down brains (chapter 4.3.4). Thus, these facts argue against the theory that the remnant structures after $T c$-ch $x$ RNAi are parts of the Central Body.

However, it is more likely that in Tc-chx RNAi the Central Body is split and misplaced. In WT larva no similar structures positive for MIP and serotonin were detected. Furthermore, the shape is rectangular and longish, exactly like the split Central Body situation after Tc-rx RNAi. That such ectopic structures appeared by chance in $T c-c h x$ RNAi is very unlikely. 
Due to the assumption that $\operatorname{ch} x$ homologues mark and specify the pars intercerebralis and that a knock down of Tc-chx leads to the loss of the regarding region, additional NBs, which are involved in Central Body formation, could be existing. Those NBs are not the putative homologue w, $x, y$, and z NBs known from Schistocerca and Drosophila (reviewed in Boyan and Reichert, 2011), but also do support the Central Body formation. The progenies of these additional NBs would not be affected by a knock down of Tc-chx and form the parts of Central Body, which are detectable in immunohistochemistry analyses for MIP and serotonin (chapter 4.3.4).

An alternative to the presence of such additional NBs is suggested by the fact that in Schistocerca, serotonergic neurons arise from the $y$ and $z$ NBs. These NB lineages form a part of the Central Complex. Boyan and colleagues (2010) identified serotonergic neurons, which occurred at stereotypic locations in their respective $y$ and $z$ lineages. In the context of the results of this work, a knock down of the respective gene did not lead to a total loss of Central Body forming NBs in the immunohistological analysis of Tc-chx RNAi brains (chapter 4.3.4). Therfore, the NB lineages forming the MIP and serotonin positive structure are not affected by Tc-chx RNAi. Eventually, to verify whether these MIP and serotonin positive structure in Tc-chx RNAi larval brains are parts of the Central Body, stainings with additional markers for the Central Body should be performed. One promising candidate could be $A P-2$, which is expressed in the Central Complex of adult Drosophila (Monge et al., 2001).

Assuming that the remnant structures occurring after Tc-chx RNAi are parts of the Central Body, the question why the Central Body is split after Tc-chx RNAi arises. One possibility is that $T c-c h x$ is involved in forming the primary axon scaffold in the brain. Whole mount in situ hybridization with an antisense probe for $T c-c h x$ revealed a strong expression pattern ventrally of a bundle of contra lateral projections (capter 4.3.1). These projections are suggested to be the onset of the protocerebral commissure (chapter 4.2). In conclusion of the expression pattern and RNAi phenotype, $T c-c h x$ is possibly important for establishing the primary axon scaffold, which enables neuronal structures to cross the brain midline. From Schistocerc, it is known that the primary axon scaffold is important for the projecting axon bundles of the Central Body NBs for orientation. The progenies, which form this axon scaffold, are located in the pars intercerebralis of Schistocerca (Boyan and Williams, 1997; Williams and Boyan, 2008). Since Tc-chx is assumed to be a pars intercerebralis marker, it is likely that the progenies forming such an axon scaffold are affected after RNAi, which finally leads to a disturbed or lacking scaffold. Therefore, the midline crossing properties are lost, which could in turn have led to the observed split Central Body phenotype in the Tc-chx RNAi animals. Whether the primary axon scaffold progenies are affected by $T c-c h x$ RNAi, 
could be tested by anti HRP staining and in this case no protocerebral commissures should be detectable.

An additional hint for $T c-c h x$ to be important for primary scaffold formation is the observed Mushroom Body phenotype after Tc-chx RNAi. This phenotype shows additional and misguided projections arising from the calyces and ending anywhere anterior in the brain. In other cases, additional projections are forming additional Pedunculi or innervate the vertical lobe of the Mushroom Body. By studying the expression pattern of the 'Brainy' line, it was revealed that the Mushroom Bodies develop late in the embryo (chapter 3.1.4). Therefore it is suggested that the Mushroom Body defects, which occur after a knock down of $T c-c h x$, are due to a secondary effect. Known from Drosophila, the developing projecting Mushroom Body tracts use the already existing neuronal scaffold as orientation (Kurusu et al., 2002; Grillenzoni et al., 2007). In this context, the Mushroom Body phenotype after Tc-chx RNAi could be caused due to scaffold defects. Therefore, additional projections as well as misguided projections arise from the Kenyon cells or calyces, because of wrong cues, which are due to a damaged or absent scaffold after Tc-chx RNAi. A direct interaction of $T c-c h x$ and the Mushroom Body NBs is unlikely, because of the distance between the Tc-chx expression domain and the position of the arising Mushroom Body NBs in the neuroectoderm. Thus it is likely, that $T c-c h x$ is involved in primary axon scaffold formation in the Tribolium brain, but it is still obscure how this occurs. Assuming that the progenies of the primary axon scaffold forming neurons are located in the pars intercerebralis like in Schistocerca (Williams and Boyan, 2008; Boyan and Williams, 2008), it is possible that these cells are affected by Tc-chx RNAi, which leads to the loss of the primary axon scaffold. On the other hand, it is also known from Schistocerca and Drosophila that glia are important for the axon guidance to establish the primary axon scaffold (Boyan et al., 1995; Lemke, 2001). Glial cells surrounding the remnants of the Central Body are missing after Tc-chx RNAi (chapter 4.3.3). Possibly, this lack of glia is actually necessary to enable the formation of the primary axon scaffold and for following midline crossing properties for projecting axon bundles of the Central Body. To what extent and what kind of glia are affected by $T c-c h x$ RNAi could be analyzed by anti Repo stainings.

Finally, this work indicates that Tc-six3 acts upstream of Tc-chx. This suggestion is based on the facts, that (1) Tc-six3 is expressed earlier than Tc-chx (chapter 4.3.1 and Posnien, 2009), (2) a knock down of Tc-six3 causes a severe cuticle RNAi phenotype, while in Tc-chx knock down no cuticle defects can be detected, that (3) in both cases a knock down lead to a loss of a properly developed Central Body, and that (4) the Tc-chx expression is lost after a knock down of Tc-six3 (Posnien, personal communication). 
Taken together, $T c$-chx was shown to be essential for proper development of the Central Body and is likely to be involved in establishing the primary axon scaffold, which gives orientation to axon tracts of the developing Central Body as well as axon tracts of the developing Mushroom Body. In addition, it was discussed to act downstream of Tc-six3.

In conclusion, $T c$-six 3 and $T c-c h x$ are very important players in specifying the cells in the median brain region, including the pars intercerebralis, in Tribolium. Additionally, these genes are important to establish the respective cues in the center of the brain, which are needed for the proper development of the Central Body as well as the Mushroom Body. Thus, this work identified two novel genes, Tc-six3 and Tc-chx, to be essential for Central Body development in Tribolium.

\subsubsection{Personal assumption of a hierarchical function of the studied genes in the context of Central Body development in Tribolium castaneum}

The here assumed antagonistic function of Tc-six 3 and Tc-otd1 was shown not to hold true. Instead, both genes seem to act on the same hierarchical level. Both genes play a crucial role in early embryonic regionalization of the anterior head in the neuroectoderm. Tc-otdl is important for the specification of the anterior part of the developing brain, which is consistent with the conserved function that was suggested for vertebrates and insvertebrates (Hirth and Reichert, 1999; Lichtneckert and Hirth, 2005; Lichtneckert and Reichert, 2008; Hirth, 2010).

Tc-six3 is important for cell proliferation and differentiation in the median anterior neuroectoderm region. During early embryonic stages, Tc-six3 maintains cell proliferation, while later on, Tc-six 3 is needed for the specification of NBs that form the Central Body. Additionally, it activates the expression of Tc-chx. Tc-chx is suggested to be involved in establishing a functional central brain glia. These glia are important to guide the outgrowing axons of the neurons, which form the primary axon scaffold. Finally, this scaffold enables the development of the Central Body.

In contrast to $T c-c h x, T c-r x$ is suggested to be required in axogenesis at the brain midline, while its target genes are unknown. Tc-rx is assumed to act on the growth cones, either by initiating the midline spanning through triggering extrinsic cues, which influence the axonal growth, or even by enabling the putative $T c-r x$ positive axons to react on extrinsic factors. As mentioned above, Tc-chx and $T c-r x$ possibly act in a synergistic fashion in order to establish the essential properties in the central midbrain, on the glial and on the axonal level. 


\subsection{Holoprosencephaly and Tribolium castaneum as model organism}

In the context of conserved processes and mechanisms among brain formation in bilaterians, the findings of this work regarding the function of $T c-s i x 3$ and $T c-c h x$ in brain formation will be discussed with respect to their relevance for the vertebrate brain. Interestingly, there are many similarities in the phenotypes in vertebrates and invertebrates caused by dysfunctions of the genes studied here during embryonic insect brain development. The vertebrate expression pattern of SIX3 starts early and marks the anterior neural plate (Oliver et al., 1995), which is similar in Tribolium (Posnien, 2009). During further development, SIX3 is expressed in the developing retina, lens, hypothalamus, and pituitary. It has been shown that hypomorphic mutations leads to a severe phenotype in mammals including humans, the holoprosencephaly. This phenotype is caused by a dysfunction in the specification of the interhemispherical region within the developing brain. In severe cases, this leads to fatal malformations where the interehemispherical fisura (fisura longitudinalis cerebri, Sobotta and Becher, 1964) is absent and no hemispherical division has taken place. Even the corpus callosum connecting the brain hemispheres is affected (reviewed in Dubourg et al., 2007). In Tribolium, a knock down of Tc-six3 leads to a loss of median tissue in the brain and finally to dramatic effects on the median head capsule, which is comparable to the phenotype of holoprosencephaly in vertebrates.

An additional gene identified in this this work was $T c-c h x$. This gene is homologous to $C H X / V S X$ in vertebrates. Here, a gene dysfunction leads to microphthalmia (Percin et al., 2000). In Tribolium, a function of Tc-chx in the larval eye development was not detected. Possibly this effect would occur in adult beetles, but a knock down of Tc-chx does not allow embryos to hatch. Nevertheless, a severe phenotype regarding the central brain in Tribolium castaneum was observed. In vertebrates, CHX-10 is expressed in the thalamus, hindbrain ventral spinal cord, and lip, but no other malformation besides that of the eyes was detected (Liu et al, 1994; Burmeister et al., 1996). Apparently, there is a redundant function, which prevents a dramatic brain phenotype. In Tribolium castaneum, there is only one homolog gene of $C H X$, which is why a knock down of this gene leads to dramatic effects within the brain.

Thus, two genes were presented and studied, both of which are conserved in bilaterians. In one case, the function is still conserved and a knock down leads to phenotypes with many similarities. In the other case, the gene expression is conserved, but the functions differ.

Finally it will be interesting, which of the 18 Tribolium homologue genes to the vertebrate neural plate genes also possess a conserved function in brain development among insects and vertebrates. With the brain visualizing system for Tribolium castaneum established in this work, this question 
could be answered. Therefore, with this work the first steps to understand the genetic processes and mechanisms of brain organogenesis for Tribolium castaneum were taken. Possibly, a genetic machinery can be identified, which gives hints to the reasons underlying malformations like holoprosencephaly. Therefore, Tribolium castaneum is suggested as an additional model organism to study homologous genes involved in brain formation and to gain insight into processes essential also for vertebrate brain development.

\subsection{Outlook}

In this work, the 'Brainy'- and 'MB'-lines were characterized regarding their expression pattern in the larval brain and the colocalisation of their signals with antibodies for glia and Mushroom Body, respectively, which had been done in cooperation with the laboratory of Schachtner (University of Marburg). These lines offer a promising tool to screen for genes which are involved in brain formation in general. Some candidate genes, which could play important roles in brain development in Tribolium, have already been found by Posnien (2009). He identified 18 genes, which are orthologues to the vertebrate neural plate genes and feature expression domains in the head neuroectoderm in Tribolium. Some of these genes showed only minor cuticle defects after RNAi, as for example $T c$-six4, and possibly their function is more specific to neural development.

However, Posnien could also show (2009), that Tc-six4 expression overlaps with Tc-six3. Thus, this gene could also be involved in Central Body formation. The opportunity to study embryonic Central Body formation in Tribolium is what distinguish this model from Drosophila, since Drosophila, one of the best established model organism for genetical studies, forms such structure only during puparium. With the 'Brainy'- and the 'MB'-line, a promising set of tools to screen for additional genes, which could be involved in or necessary for proper Central Body formation in Tribolium, was created.

The generated Tc-rx reporter lines show a defined expression pattern, but still it is not clear whether the detected cells positive for the reportergene expression arose from $T c-r x$ positive NBs. One option to answer this question, could be a colocalisation experiment with the reportergene and $T c$ $r x$. Assuming, that the reporter lines 'Tc-rx 5up::DsRedEx' and 'Tc-rx Intron::DsRedEx' are marking Tc-rx NB lineages, these lines offer the opportunity to study the selection of NB identity by RNAi with candidate genes. Parallel to that, additional NB lineage marking lines could be generated. One reporter gene construct for $T c-r x$ is still missing. In addition, new constructs for $T c$ chx could be designed and used to generate NB reporter lines. 
In this work, it could be shown that $T c-r x, T c-c h x$, and $T c$-six 3 are important for proper Central Body development. In the regarding paragraph, different opportunities and putative roles in Central Body formation were discussed. The target genes of $T c-r x$, $T c-c h x$, and $T c-s i x 3$ are still unknown, but these could be revealed by chromatin immunoprecipitation (Chip; Orlando et al., 1997; Orlando, 2000) with regarding antibodies. By this, further candidate genes for creating additional lines could be identified. Furthermore, the identification of the target genes of $T c-r x$, Tc-chx and Tcsix 3 could deliver additional valuable details regarding Central Body development. 


\section{References}

Acampora, D., Avantaggiato, V., Tuorto, F., and Simeone, A. (1997). Genetic control of brain morphogenesis through Otx gene dosage requirement. Development 124, 3639-650.

Acampora, D., Avantaggiato, V., Tuorto, F., Barone, P., Reichert, H., Finkelstein, R., and Simeone, A. (1998). Murine Otx1 and Drosophila otd genes share conserved genetic functions required in invertebrate and vertebrate brain development. Development 125, 1691.

Acampora, D., Gulisano, M., and Simeone, A. (1999). Otx genes and the genetic control of brain morphogenesis. Mol Cell Neurosci 13, 1-8.

Acampora, D., Gulisano, M., Broccoli, V., and Simeone, A. (2001). Otx genes in brain morphogenesis. Prog Neurobiol 64, 69-95.

Acampora, D., Mazan, S., Lallemand, Y., Avantaggiato, V., Maury, M., Simeone, A., and Brûlet, P. (1995). Forebrain and midbrain regions are deleted in Otx2-/- mutants due to a defective anterior neuroectoderm specification during gastrulation. Development 121, 3279-290.

Andreazzoli, M., Pannese, M., and Boncinelli, E. (1997). Activating and repressing signals in head development: the role of Xotx1 and Xotx2. Development 124, 1733-743.

Arendt, D., and Nübler-Jung, K. (1994). Inversion of dorsoventral axis? 371, 26.

Arendt, D., and Nübler-Jung, K. (1999). Comparison of early nerve cord development in insects and vertebrates. Development 126, 2309-325.

Arendt, D., Denes, A.S., Jékely, G., and Tessmar-Raible, K. (2008). The evolution of nervous system centralization. Philos Trans R Soc Lond B Biol Sci 363, 1523-28.

Arendt, D., Tessmar, K., de Campos-Baptista, M.I., Dorresteijn, A., and Wittbrodt, J. (2002). Development of pigment-cup eyes in the polychaete Platynereis dumerilii and evolutionary conservation of larval eyes in Bilateria. Development 129, 1143-154.

Arendt, D., Tessmar-Raible, K., Snyman, H., Dorresteijn, A.W., and Wittbrodt, J. (2004). Ciliary photoreceptors with a vertebrate-type opsin in an invertebrate brain. Science 306, 869.

Awasaki, T., Lai, S. L., Ito, K., \& Lee, T. (2008). Organization and postembryonic development of glial cells in the adult central brain of drosophila. The Journal of Neuroscience : The Official Journal of the Society for Neuroscience, 28(51), 13742-53. doi:10.1523/JNEUROSCI. 4844-08.2008

Axelrod, J. D. (2010). Delivering the lateral inhibition punchline: It's all about the timing. Science Signaling, 3(145)

Ayali, A., and Lange, A.B. (2010). Rhythmic behaviour and pattern-generating circuits in the locust: Key concepts and recent updates. Journal of Insect Physiology 56, 834-843. 
Badisco, L., Huybrechts, J., Simonet, G., Verlinden, H., Marchal, E., Huybrechts, R., Schoofs, L., De Loof, A., and Vanden Broeck, J. (2011). Transcriptome Analysis of the Desert Locust Central Nervous System: Production and Annotation of a Schistocerca gregaria EST Database. PLoS One 6, e17274.

Bailey, T.J., El-Hodiri, H., Zhang, L., Shah, R., Mathers, P.H., and Jamrich, M. (2004). Regulation of vertebrate eye development by Rx genes. Int J Dev Biol 48, 761-770.

Balciunas, D., Davidson, A.E., Sivasubbu, S., Hermanson, S.B., Welle, Z., and Ekker, S.C. (2004). Enhancer trapping in zebrafish using the Sleeping Beauty transposon. BMC Genomics 5, 62.

Beatus, P., and Lendahl, U. (1998). Notch and neurogenesis. J Neurosci Res 54, 125-136.

Bentley, D., and Toroian-Raymond, A. (1981). Embryonic and postembryonic morphogenesis of a grasshopper interneuron. J Comp Neurol 201, 507-518.

Berger, C., Renner, S., Lüer, K., and Technau, G.M. (2007). The commonly used marker ELAV is transiently expressed in neuroblasts and glial cells in the Drosophila embryonic CNS. Dev Dyn 236, 3562-68.

Berghammer, A.J., Klingler, M., and Wimmer, E.A. (1999). A universal marker for transgenic insects. Nature 402, 370-71.

Berghammer, A.J., Weber, M., Trauner, J., and Klingler, M. (2009). Red flour beetle (Tribolium) germline transformation and insertional mutagenesis. Cold Spring Harb Protoc 2009, pdb.prot5259.

Bishop, K.M., Rubenstein, J.L., and O'Leary, D.D. (2002). Distinct actions of Emx1, Emx2, and Pax6 in regulating the specification of areas in the developing neocortex. J Neurosci 22, 7627-638.

Blanco, J., Seimiya, M., Pauli, T., Reichert, H., and Gehring, W.J. (2009). Wingless and Hedgehog signaling pathways regulate orthodenticle and eyes absent during ocelli development in Drosophila. Dev Biol 329, 104 - 115.

Boyan, G., Herbert, Z., and Williams, L. (2010). Cell death shapes embryonic lineages of the Central Complex in the grasshopper Schistocerca gregaria. J Morphol 271, 949-959.

Boyan, G.S., and Reichert, H. (2011). Mechanisms for complexity in the brain: generating the insect Central Complex. Trends Neurosci

Boyan, G.S., and Williams, J.L.D. (1997). Embryonic development of the pars intercerebralis/ Central Complex of the grasshopper. Dev Genes Evol 207, 317-329.

Boyan, G.S., Williams, J.L.D., and Reichert, H. (1995). Morphogenetic reorganization of the brain during embryogenesis in the grasshopper. J Comp Neurol 361, 429-440. 
Boyan, G.S., Williams, J.L., Posser, S., and Bräunig, P. (2002). Morphological and molecular data argue for the labrum being non-apical, articulated, and the appendage of the intercalary segment in the locust. Arthropod Struct Dev 31, 65-76.

Braitenberg, V., Schüz, A., Braitenberg, V., Sabin, S., Gahse, Z., Braitenberg, V., Braitenberg, V., Braitenberg, V., Aertsen, A., and Palm, G. (1998). Cortex: statistics and geometry of neuronal connectivity. New York: Berlin

Brand, M., Jarman, A. P., Jan, L. Y., \& Jan, Y. N. (1993). Asense is a drosophila neural precursor gene and is capable of initiating sense organ formation. Development (Cambridge, England), $119(1), 1-17$.

Brasset, E., and Vaury, C. (2005). Insulators are fundamental components of the eukaryotic genomes. Heredity 94, 571-76.

Bretschneider, F. (1914). Über die Gehirne der Küchenschabe und des Mehlkäfers (Kämpfe).

Brose, K., Bland, K.S., Wang, K.H., Arnott, D., Henzel, W., Goodman, C.S., Tessier-Lavigne, M., and Kidd, T. (1999). Slit proteins bind Robo receptors and have an evolutionarily conserved role in repulsive axon guidance. Cell 96, 795-806.

Brown, S.J., Mahaffey, J.P., Lorenzen, M.D., Denell, R.E., and Mahaffey, J.W. (1999). Using RNAi to investigate orthologous homeotic gene function during development of distantly related insects. Evol Dev 1, 11-15.

Brown, S.J., Shippy, T.D., Miller, S., Bolognesi, R., Beeman, R.W., Lorenzen, M.D., Bucher, G., Wimmer, E.A., and Klingler, M. (2009). The red flour beetle, Tribolium castaneum (Coleoptera): a model for studies of development and pest biology. Cold Spring Harb Protoc 2009, pdb.emo126.

Bucher, G., Scholten, J., and Klingler, M. (2002). Parental RNAi in Tribolium (Coleoptera). Curr Biol 12, R85.

Bullock, T.H., Horridge, G.A., Bern, H.A., Hagadorn, I.R., and Smith, J.E. (1965). Structure and function in the nervous systems of invertebrates.

Burrows, M., and NetLibrary, I. (1996). The neurobiology of an insect brain (Oxford University Press Oxford, UK:).

Bushey, A.M., Dorman, E.R., and Corces, V.G. (2008). Chromatin insulators: regulatory mechanisms and epigenetic inheritance. Mol Cell 32, 1-9.

Cardona, A., Saalfeld, S., Preibisch, S., Schmid, B., Cheng, A., Pulokas, J., Tomancak, P., and Hartenstein, V. (2010). An integrated micro- and macroarchitectural analysis of the Drosophila brain by computer-assisted serial section electron microscopy. PLoS Biol 8 
Chuang, J.C., Mathers, P.H., and Raymond, P.A. (1999). Expression of three Rx homeobox genes in embryonic and adult zebrafish. Mech Dev 84, 195-98.

Colonques, J., Ceron, J., and Tejedor, F.J. (2007). Segregation of postembryonic neuronal and glial lineages inferred from a mosaic analysis of the Drosophila larval brain. Mech Dev 124, 327-340.

Del Bene, F.D., Tessmar-Raible, K., Wittbrodt, J., Bene, F.D., Tessmar-Raible, K., and Wittbrodt, J. (2004). Direct interaction of geminin and Six3 in eye development. Nature 427, 745.

Denes, A.S., Jékely, G., Steinmetz, P.R., Raible, F., Snyman, H., Prud'homme, B., Ferrier, D.E., Balavoine, G., and Arendt, D. (2007). Molecular architecture of annelid nerve cord supports common origin of nervous system centralization in bilateria. Cell 129, 277-288.

Deschet, K., Bourrat, F., Ristoratore, F., Chourrout, D., and Joly, J.S. (1999). Expression of the medaka (Oryzias latipes) Ol-Rx3 paired-like gene in two diencephalic derivatives, the eye and the hypothalamus. Mech Dev 83, 179-182.

Dettner, K., Peters, W., and Bauer, T. (2003). Lehrbuch der Entomologie (Spektrum Akademischer Verlag).

Dickson, B.J., and Gilestro, G.F. (2006). Regulation of commissural axon pathfinding by slit and its Robo receptors. Annu Rev Cell Dev Biol 22, 651-675.

Doe, C.Q., and Goodman, C.S. (1985). Early events in insect neurogenesis : II. The role of cell interactions and cell lineage in the determination of neuronal precursor cells. Dev Biol 111, 206 $-219$.

Dong, Y., and Friedrich, M. (2005). Nymphal RNAi: systemic RNAi mediated gene knockdown in juvenile grasshopper. BMC Biotechnol 5, 25.

Dong, Y., and Friedrich, M. (2010). Enforcing biphasic eye development in a directly developing insect by transient knockdown of single eye selector genes. J Exp Zool B Mol Dev Evol 314B, 104-114.

Dreyer, D., Vitt, H., Dippel, S., Goetz, B., El Jundi, B., Kollmann, M., Huetteroth, W., and Schachtner, J. (2010). 3D Standard Brain of the Red Flour Beetle Tribolium Castaneum: A Tool to Study Metamorphic Development and Adult Plasticity. Front Syst Neurosci 4, 3.

Dubourg, C., Bendavid, C., Pasquier, L., Henry, C., Odent, S., and David, V. (2007). Holoprosencephaly. Orphanet J Rare Dis 2, 8.

Edenfeld, G., Stork, T., and Klämbt, C. (2005). Neuron-glia interaction in the insect nervous system. Curr Opin Neurobiol 15, 34-39.

Edwards, T.N., and Meinertzhagen, I.A. (2010). The functional organisation of glia in the adult 
brain of Drosophila and other insects. Prog Neurobiol 90, 471-497.

Eggert, T., Hauck, B., Hildebrandt, N., Gehring, W.J., and Walldorf, U. (1998). Isolation of a

Drosophila homolog of the vertebrate homeobox gene $\mathrm{Rx}$ and its possible role in brain and eye development. Proc Natl Acad Sci U S A 95, 2343-48.

El Jundi, B., Heinze, S., Lenschow, C., Kurylas, A., Rohlfing, T., and Homberg, U. (2009). The Locust Standard Brain: A 3D Standard of the Central Complex as a Platform for Neural Network Analysis. Front Syst Neurosci 3, 21.

El Jundi, B.E., and Homberg, U. (2010). Evidence for the possible existence of a second polarization-vision pathway in the locust brain. Journal of Insect Physiology 56, 971 - 979.

Elphick, M., Williams, L., and Shea, M. (1996). New features of the locust optic lobe: evidence of a role for nitric oxide in insect vision. J Exp Biol 199, 2395-2407.

Erclik, T., Hartenstein, V., Lipshitz, H.D., and McInnes, R.R. (2008). Conserved role of the Vsx genes supports a monophyletic origin for bilaterian visual systems. Curr Biol 18, 1278-287.

Evans, T.A., and Bashaw, G.J. (2010). Axon guidance at the midline: of mice and flies. Curr Opin Neurobiol 20, 79 - 85 .

Fahrbach, S.E. (2006). Structure of the mushroom bodies of the insect brain. Annu Rev Entomol 51, 209-232.

Finkelstein, R., and Boncinelli, E. (1994). From fly head to mammalian forebrain: the story of otd and Otx. Trends Genet 10,310-15.

Finkelstein, R., Smouse, D., Capaci, T.M., Spradling, A.C., and Perrimon, N. (1990). The orthodenticle gene encodes a novel homeo domain protein involved in the development of the Drosophila nervous system and ocellar visual structures. Genes Dev 4, 1516-527.

Flögel, J.H.L. (1876). Über den feineren Bau des Arthropodengehirns. Tagbl. Versamml. dtschr. Naturforsch. Ärzte.(Beilage) 49, 115-120.

Flögel, J.H.L. (1878). Über den einheitlichen Bau des Gehirns in den verschiedenen Insektenordnungen. Z Wissen Zool (Suppl) 30, 556-592.

Frantz, G.D., Weimann, J.M., Levin, M.E., and McConnell, S.K. (1994). Otx 1 and Otx2 define layers and regions in developing cerebral cortex and cerebellum. J Neurosci 14, 5725.

Fung, S., Wang, F., Spindler, S.R., and Hartenstein, V. (2009). Drosophila E-cadherin and its binding partner Armadillo/ beta-catenin are required for axonal pathway choices in the developing larval brain. Dev Biol 332, 371-382.

Furrer, M.P., Vasenkova, I., Kamiyama, D., Rosado, Y., and Chiba, A. (2007). Slit and Robo control the development of dendrites in Drosophila CNS. Development 134, 3795-3804. 
Gan, L., Liu, X., Xiang, Z., and He, N. (2011). Microarray-based gene expression profiles of silkworm brains. BMC Neurosci 12, 8 .

Gavrieli, Y., Sherman, Y., and Ben-Sasson, S.A. (1992). Identification of programmed cell death in situ via specific labeling of nuclear DNA fragmentation. J Cell Biol 119, 493-501.

Geng, X., Speirs, C., Lagutin, O., Inbal, A., Liu, W., Solnica-Krezel, L., Jeong, Y., Epstein, D.J., and Oliver, G. (2008). Haploinsufficiency of Six3 fails to activate Sonic hedgehog expression in the ventral forebrain and causes holoprosencephaly. Dev Cell 15, 236-247.

Gestri, G., Carl, M., Appolloni, I., Wilson, S.W., Barsacchi, G., and Andreazzoli, M. (2005). Six3 functions in anterior neural plate specification by promoting cell proliferation and inhibiting Bmp4 expression. Development 132, 2401-413.

Gilbert, S.F. (2003). Dev Biol (Sunderland, Mass.: Sinauer Associates).

Grillenzoni, N., Flandre, A., Lasbleiz, C., and Dura, J.M. (2007). Respective roles of the DRL receptor and its ligand WNT5 in Drosophila Mushroom Body development. Development 134, 3089-097.

Groth, A.C., and Calos, M.P. (2004). Phage integrases: biology and applications. Journal of molecular biology 335, 667-678.

Halter, D.A., Urban, J., Rickert, C., Ner, S.S., Ito, K., Travers, A.A., and Technau, G.M. (1995). The homeobox gene repo is required for the differentiation and maintenance of glia function in the embryonic nervous system of Drosophila melanogaster. Development 121, 317.

Handel, K., Basal, A., Fan, X., and Roth, S. (2005). Tribolium castaneum twist: gastrulation and mesoderm formation in a short-germ beetle. Dev Genes Evol 215, 13-31.

Hanström, B. (1925). Comparison between the brains of the newly hatched larva and the imago of Pieris brassicae. Ent. Tidskr 46, 43-52.

Hartenstein, V. (2006). The neuroendocrine system of invertebrates: a developmental and evolutionary perspective. J Endocrinol 190, 555-570.

Hartenstein, V., Nassif, C., and Lekven, A. (1998). Embryonic development of the Drosophila brain. II. Pattern of glial cells. J Comp Neurol 402, 32-47.

Hartenstein, V., Rudloff, E., and Campos -Ortega, J.A. (1987). The pattern of proliferation of the neuroblasts in the wild-type embryo of Drosophila melanogaster $\leq i>$. Dev Genes Evol 196, 473-485.

Hartenstein, V., Spindler, S., Pereanu, W., and Fung, S. (2008). The development of the Drosophila larval brain. Brain development in Drosophila melanogaster , 1-31.

Hein, H., (2007). Identifizierung von Neuroblasten als Modell für die Regulierung ihrer 
Spezifizierung in Tribolium castaneum. Diploma Thesis.

Heinze, S., and Homberg, U. (2009). Linking the input to the output: new sets of neurons complement the polarization vision network in the locust central complex. J Neurosci 29, 4911-921.

Hendzel, M.J., Wei, Y., Mancini, M.A., Van Hooser, A., Ranalli, T., Brinkley, B.R., Bazett-Jones, D.P., and Allis, C.D. (1997). Mitosis-specific phosphorylation of histone H3 initiates primarily within pericentromeric heterochromatin during G2 and spreads in an ordered fashion coincident with mitotic chromosome condensation. Chromosoma 106, 348-360.

Herbert, Z., Rauser, S., Williams, L., Kapan, N., Güntner, M., Walch, A., and Boyan, G. (2010). Developmental expression of neuromodulators in the Central Complex of the grasshopper Schistocerca gregaria. J Morphol 271, 1509-526.

Hirth, F. (2010). On the origin and evolution of the tripartite brain. Brain Behav Evol 76, 3-10.

Hirth, F., and Reichert, H. (1999). Conserved genetic programs in insect and mammalian brain development. BioEssays 21, 677-684.

Hirth, F., Therianos, S., Loop, T., Gehring, W.J., Reichert, H., and Furukubo-Tokunaga, K. (1995). Developmental defects in brain segmentation caused by mutations of the homeobox genes orthodenticle and empty spiracles in Drosophila. Neuron 15, 769-778.

Hofbauer, A., and Campos-Ortega, J.A. (1990). Proliferation pattern and early differentiation of the optic lobes in Drosophila melanogaster. Dev Genes Evol 198, 264-274.

Holland, N.D., and Holland, N.D. (2003). Early central nervous system evolution: an era of skin brains? Nat Rev Neurosci 4, 617.

Homberg, U. (2008). Evolution of the Central Complex in the arthropod brain with respect to the visual system. Arthropod Struct Dev 37, 347-362.

Homberg, U., and Hildebrand, J.G. (1994). Postembryonic development of $\gamma$-aminobutyric acid-like Immunoreactivity in the brain of the sphinx moth Manduca sexta. J Comp Neurol 339, 132-149.

Homberg, U., Hofer, S., Pfeiffer, K., and Gebhardt, S. (2003). Organization and neural connections of the anterior optic tubercle in the brain of the locust, Schistocerca gregaria. J Comp Neurol 462, 415-430.

Hong, S.-T., Bang, S., Hyun, S., Kang, J., Jeong, K., Paik, D., Chung, J., Kim, J. (2008). cAMP signalling in mushroom bodies modulates temperature preference behaviour in Drosophila. Nature 454, 771.

Horn, C., Jaunich, B., and Wimmer, E.A. (2000). Highly sensitive, fluorescent transformation marker for Drosophila transgenesis. Dev Genes Evol 210, 623-29. 
Huetteroth, W., El Jundi, B., El Jundi, S., and Schachtner, J. (2010). 3D-reconstructions and virtual 4D-visualization to study metamorphic brain development in the sphinx moth Manduca sexta. Front Syst Neurosci 4

Ito, K., and Hotta, Y. (1992). Proliferation pattern of postembryonic neuroblasts in the brain of Drosophila melanogaster. Dev Biol 149, 134 - 148.

Izergina, N., Balmer, J., Bello, B., and Reichert, H. (2009). Postembryonic development of transit amplifying neuroblast lineages in the Drosophila brain. Neural Dev 4, 44.

Jan, L.Y., and Jan, Y.N. (1982). Antibodies to horseradish peroxidase as specific neuronal markers in Drosophila and in grasshopper embryos. Proc Natl Acad Sci U S A 79, 2700-04.

JawLowski, H. (1936). Ueber den gehirnbau der kaefer. Zeitschrift fuer Morphologie und Oekologie der Tiere 32, 67-91.

Jen, J. C., Chan, W. -M., Bosley, T. M., Wan, J., Carr, J. R., Rüb, U., et al.. (2004). Mutations in a human ROBO gene disrupt hindbrain axon pathway crossing and morphogenesis. Science (New York, N.Y.), 304(5676)

Johansen, H. (1892). Die Entwicklung des Imagoauges von Vanessa urticae L. Zool. Jahrb., Abt. f. Anat. u. Ontog., Bd 6

Johard, H.A., Enell, L.E., Gustafsson, E., Trifilieff, P., Veenstra, J.A., and Nässel, D.R. (2008). Intrinsic neurons of Drosophila mushroom bodies express short neuropeptide F: relations to extrinsic neurons expressing different neurotransmitters. J Comp Neurol 507, 1479-496.

Kammermeier, L., and Reichert, H. (2001). Common developmental genetic mechanisms for patterning invertebrate and vertebrate brains. Brain Res Bull 55, 675-682.

Kent, K.S., Hoskins, S.G., and Hildebrand, J.G. (1987). A novel serotonin-immunoreactive neuron in the antennal lobe of the sphinx moth Manduca sexta persists throughout postembryonic life. Journal of Neurobiology 18, 451-465.

Kenyon, F.C. (1896). The meaning and structure of the so-called" mushroom bodies" of the hexapod brain. The American Naturalist 30, 643-650.

Klagges, B.R.E., Heimbeck, G., Godenschwege, T.A., Hofbauer, A., Pflugfelder, G.O., Reifegerste, R., Reisch, D., Schaupp, M., Buchner, S., and Buchner, E. (1996). Invertebrate synapsins: a single gene codes for several isoforms in Drosophila. Journal of Neuroscience 16, 3154.

Klämbt, C., and Goodman, C.S. (1991). The diversity and pattern of glia during axon pathway formation in the drosophila embryo. Glia 4, 205-213.

Kobayashi, M., Toyama, R., Takeda, H., Dawid, I.B., and Kawakami, K. (1998). Overexpression of the forebrain-specific homeobox gene six 3 induces rostral forebrain enlargement in zebrafish. 
Development 125, 2973-982.

Kotkamp, K., Klingler, M., and Schoppmeier, M. (2010). Apparent role of Tribolium orthodenticle in anteroposterior blastoderm patterning largely reflects novel functions in dorsoventral axis formation and cell survival. Development 137, 1853-862.

Kumar, A., Fung, S., Lichtneckert, R., Reichert, H., and Hartenstein, V. (2009). Arborization pattern of engrailed-positive neural lineages reveal neuromere boundaries in the Drosophila brain neuropil. J Comp Neurol 517, 87-104.

Kurosaka, A., Yano, A., Itoh, N., Kuroda, Y., Nakagawa, T., and Kawasaki, T. (1991). The structure of a neural specific carbohydrate epitope of horseradish peroxidase recognized by antihorseradish peroxidase antiserum. J Biol Chem 266, 4168-172.

Kurusu, M., Awasaki, T., Masuda-Nakagawa, L.M., Kawauchi, H., Ito, K., and Furukubo-Tokunaga, K. (2002). Embryonic and larval development of the Drosophila mushroom bodies: concentric layer subdivisions and the role of fasciclin II. Development 129, 409-419.

Kurylas, A.E., Rohlfing, T., Krofczik, S., Jenett, A., and Homberg, U. (2008). Standardized atlas of the brain of the desert locust, Schistocerca gregaria. Cell Tissue Res 333, 125-145.

Kwon, D., Mucci, D., Langlais, K.K., Americo, J.L., DeVido, S.K., Cheng, Y., and Kassis, J.A. (2009). Enhancer-promoter communication at the Drosophila engrailed locus. Development 136, 3067-075.

Lagrange, T., Kapanidis, A.N., Tang, H., Reinberg, D., and Ebright, R.H. (1998). New core promoter element in RNA polymerase II-dependent transcription: sequence-specific DNA binding by transcription factor IIB. Genes Dev 12, 34-44.

Lane, M.E., and Kalderon, D. (1993). Genetic investigation of cAMP-dependent protein kinase function in Drosophila development. Genes Dev 7, 1229.

Larsen, C., Shy, D., Spindler, S.R., Fung, S., Pereanu, W., Younossi-Hartenstein, A., and Hartenstein, V. (2009). Patterns of growth, axonal extension and axonal arborization of neuronal lineages in the developing Drosophila brain. Dev Biol 335, 289-304.

Lee, B.P., and Jones, B.W. (2005). Transcriptional regulation of the Drosophila glial gene repo. Mech Dev 122, 849-862.

Leuzinger, S., Hirth, F., Gerlich, D., Acampora, D., Simeone, A., Gehring, W.J., Finkelstein, R., Furukubo-Tokunaga, K., and Reichert, H. (1998). Equivalence of the fly orthodenticle gene and the human OTX genes in embryonic brain development of Drosophila. Development 125 , 1703-710.

Lichtneckert, R., and Reichert, H. (2005). Insights into the urbilaterian brain: conserved genetic 
patterning mechanisms in insect and vertebrate brain development. Heredity 94, 465-477.

Lichtneckert, R., and Reichert, H. (2008). Anteroposterior regionalization of the brain: genetic and comparative aspects. Brain development in Drosophila melanogaster, 32-41.

Liu, G., Seiler, H., Wen, A., Zars, T., Ito, K., Wolf, R., Heisenberg, M., and Liu, L. (2006). Distinct memory traces for two visual features in the Drosophila brain. Nature 439, 551-56.

Livet, J., Weissman, T.A., Kang, H., Draft, R.W., Lu, J., Bennis, R.A., Sanes, J.R., and Lichtman, J.W. (2007). Transgenic strategies for combinatorial expression of fluorescent proteins in the nervous system. Nature 450, 56-62.

Loesel, R., Nässel, D.R., and Strausfeld, N.J. (2002). Common design in a unique midline neuropil in the brains of arthropods. Arthropod Struct Dev 31, 77-91.

Loosli, F., Köster, R.W., Carl, M., Krone, A., and Wittbrodt, J. (1998). Six3, a medaka homologue of the Drosophila homeobox gene sine oculis is expressed in the anterior embryonic shield and the developing eye. Mech Dev 74, 159-164.

Loosli, F., Staub, W., Finger-Baier, K.C., Ober, E.A., Verkade, H., Wittbrodt, J., and Baier, H. (2003). Loss of eyes in zebrafish caused by mutation of chokh/rx3. EMBO Rep 4, 894-99.

Loosli, F., Winkler, S., and Wittbrodt, J. (1999). Six3 overexpression initiates the formation of ectopic retina. Genes Dev 13, 649-654.

Lowe, C.J., Wu, M., Salic, A., Evans, L., Lander, E., Stange-Thomann, N., Gruber, C.E., Gerhart, J., and Kirschner, M. (2003). Anteroposterior patterning in hemichordates and the origins of the chordate nervous system. Cell 113, 853-865.

Ludwig, P., Williams, L., and Boyan, G. (2002). The pars intercerebralis of the locust brain: a developmental and comparative study. Microsc Res Tech 56, 174-188.

Lynch, J.A., Brent, A.E., Leaf, D.S., Pultz, M.A., Desplan, C., Lynch, J.A., Brent, A.E., Leaf, D.S., Pultz, M.A., and Desplan, C. (2006). Localized maternal orthodenticle patterns anterior and posterior in the long germ wasp Nasonia. Nature 439, 728.

Martin, J.-, Raabe, T., and Heisenberg, M. (1999). Central Complex substructures are required for the maintenance of locomotor activity in Drosophila melanogaster. J Comp Physiol A Neuroethol Sens Neural Behav Physiol 185, 277-288.

Mathers, P.H., Grinberg, A., Mahon, K.A., Jamrich, M., Mathers, P.H., Grinberg, A., Mahon, K.A., and Jamrich, M. (1997). The Rx homeobox gene is essential for vertebrate eye development. Nature 387, 603.

Meier, T., Therianos, S., Zacharias, D., and Reichert, H. (1993). Developmental expression of TERM-1 glycoprotein on growth cones and terminal arbors of individual identified neurons in 
the grasshopper. J Neurosci 13, 1498-1510.

Monge, I., Krishnamurthy, R., Sims, D., Hirth, F., Spengler, M., Kammermeier, L., Reichert, H., and Mitchell, P.J. (2001). Drosophila transcription factor AP-2 in proboscis, leg and brain Central Complex development. Development 128, 1239-252.

Nässel, D.R. (2002). Neuropeptides in the nervous system of Drosophila and other insects: multiple roles as neuromodulators and neurohormones. Prog Neurobiol 68, 1-84.

Nicolas, E., and Preat, T. (2005). Drosophila central brain formation requires Robo proteins. Dev Genes Evol 215, 530-36.

Noveen, A., Daniel, A., and Hartenstein, V. (2000). Early development of the Drosophila Mushroom Body: the roles of eyeless and dachshund. Development 127, 3475-488.

Ohuchi, H., Tomonari, S., Itoh, H., Mikawa, T., and Noji, S. (1999). Identification of chick rax/rx genes with overlapping patterns of expression during early eye and brain development. Mech Dev 85, 193-95.

Orlando, V., Strutt, H., and Paro, R. (1997). Analysis of chromatin structure by in vivo formaldehyde cross-linking. Methods 11, 205-214.

Orlando, V. (2000). Mapping chromosomal proteins in vivo by formaldehyde-crosslinkedchromatin immunoprecipitation. Trends Biochem Sci 25, 99-104.

Panov, A.A. (1959). Structure of the insect brain at successive stages of postembryonic development. II. The Central Body. Entomol Rev URSS 38, 276-284.

Parker, R.J., and Auld, V.J. (2006). Roles of glia in the Drosophila nervous system. Semin Cell Dev Biol 17, 66-77.

Percin, E.F., Ploder, L.A., Yu, J.J., Arici, K., Horsford, D.J., Rutherford, A., Bapat, B., Cox, D.W., Duncan, A.M., et al. (2000). Human microphthalmia associated with mutations in the retinal homeobox gene CHX10. Nat Genet 25, 397.

Pereanu, W., Younossi-Hartenstein, A., Lovick, J., Spindler, S., and Hartenstein, V. (2011). Lineagebased analysis of the development of the Central Complex of the drosophila brain. J Comp Neurol 519, 661-689.

Pfeiffer, K., Kinoshita, M., and Homberg, U. (2005). Polarization-sensitive and light-sensitive neurons in two parallel pathways passing through the anterior optic tubercle in the locust brain. J Neurophysiol 94, 3903-915.

Pi, H., and Chien, C.-T. (2007). Getting the edge: neural precursor selection. Journal of Biomedical Science $14,467-473$.

Poeck, B., Triphan, T., Neuser, K., and Strauss, R. (2008). Locomotor control by the Central 
Complex in Drosophila-An analysis of the tay bridge mutant. Developmental Neurobiology $68,1046-058$.

Portin, P. (2002). General outlines of the molecular genetics of the Notch signalling pathway in Drosophila melanogaster: a review. Hereditas 136, 89-96.

Posnien, N. (2006). Das tribolium castaneum gen tc'optix/six3 und die evolution des labrum. Diploma Thesis

Posnien, N. (2009). Function and evolution of highly conserved head genes in the red flour beetle tribolium castaneum. $\mathrm{PhD}$-Thesis

Posnien, N., Schinko, J., Grossmann, D., Shippy, T.D., Konopova, B., and Bucher, G. (2009). RNAi in the red flour beetle (Tribolium). Cold Spring Harb Protoc 2009, pdb.prot5256.

Predel, R., Rapus, J., and Eckert, M. (2001). Myoinhibitory neuropeptides in the American cockroach. Peptides 22, 199-208.

Prillinger, L. (1981). Postembryonic development of the Antennal Lobes in Periplaneta americana L. Cell Tissue Res 215, 563-575.

Prpic, N.M., and Damen, W.G. (2005). Cell death during germ band inversion, dorsal closure, and nervous system development in the spider Cupiennius salei. Dev Dyn 234, 222-28.

Prpic, N.M., Wigand, B., Damen, W.G., and Klingler, M. (2001). Expression of dachshund in wildtype and Distal-less mutant Tribolium corroborates serial homologies in insect appendages. Dev Genes Evol 211, 467-477.

Reichert, H. (2009). Evolutionary conservation of mechanisms for neural regionalization, proliferation and interconnection in brain development. Biol Lett 5, 112-16.

Reichert, H., and Boyan, G. (1997). Building a brain: developmental insights in insects. Trends Neurosci 20, 258-264.

Renn, S.C.P., Armstrong, J.D., Yang, M., Wang, Z., An, X., Kaiser, K., and Taghert, P.H. (1999). Genetic analysis of the Drosophila ellipsoid body neuropil: Organization and development of the Central Complex. Journal of Neurobiology 41, 189-207.

Richards, S., Gibbs, R.A., Weinstock, G.M., Brown, S.J., Denell, R., Beeman, R.W., Gibbs, R., Beeman, R.W., Brown, S.J., et al. (2008). The genome of the model beetle and pest Tribolium castaneum. Nature 452, 949-955.

Rojas-Muñoz, A., Dahm, R., and Nüsslein-Volhard, C. (2005). chokh/rx3 specifies the retinal pigment epithelium fate independently of eye morphogenesis. Dev Biol 288, 348 - 362.

Rothberg, J.M., Jacobs, J.R., Goodman, C.S., and Artavanis-Tsakonas, S. (1990). slit: an extracellular protein necessary for development of midline glia and commissural axon pathways 
contains both EGF and LRR domains. Genes Dev 4, 2169.

Rubin, G. M., Yandell, M. D., Wortman, J. R., Gabor, G. L., Miklos, Nelson, C. R., Hariharan, I. K., Fortini, M. E., Li, P.W., et. al. (2000). Comparative genomics of the eukaryotes. Science (New York, N.Y.), 287(5461), 2204. doi:10.1126/science.287.5461.2204

Samson, M.L., and Chalvet, F. (2003). found in neurons, a third member of the Drosophila elav gene family, encodes a neuronal protein and interacts with elav. Mech Dev 120, 373-383.

Schetelig, M.F., Schmid, B.G.M., Zimowska, G., and Wimmer, E.A. (2008). Plasticity in mRNA expression and localization of orthodenticle within higher Diptera. Evol Dev 10, 700-04.

Schinko, J., Posnien, N., Kittelmann, S., Koniszewski, N., and Bucher, G. (2009). Single and double whole-mount in situ hybridization in red flour beetle (Tribolium) embryos. Cold Spring Harb Protoc 2009

Schinko, J.B., Kreuzer, N., Offen, N., Posnien, N., Wimmer, E.A., and Bucher, G. (2008). Divergent functions of orthodenticle, empty spiracles and buttonhead in early head patterning of the beetle Tribolium castaneum (Coleoptera). Dev Biol 317, 600-613.

Schmidt-Ott, U., and Martin Technau, G. (1994). Fate-mapping in the procephalic region of the embryonic Drosopbila head. Dev Genes Evol 203, 367-373.

Schrader, K. (1938). Untersuchungen über die Normalentwicklung des Gehirns und Gehirntransplantationen bei der Mehlmotte Ephestia kühniella Zeller nebst einigen Bemerkungen über das Corpus allatum (Göttingen).

Schröder, R. (2003). The genes orthodenticle and hunchback substitute for bicoid in the beetle Tribolium. Nature 422, 621-25.

Seimiya, M., and Gehring, W.J. (2000). The Drosophila homeobox gene optix is capable of inducing ectopic eyes by an eyeless-independent mechanism. Development 127, 1879-886.

Sharma, V.M., Ruiz de Luzuriaga, A.M., Waggoner, D., Greenwald, M., and Stein, S.L. (2008). Microphthalmia with linear skin defects: a case report and review. Pediatr Dermatol 25, 548-552.

Simeone, A., Acampora, D., Gulisano, M., Stornaiuolo, A., and Boncinelli, E. (1992). Nested expression domains of four homeobox genes in developing rostral brain. Nature 358, 687-690.

Simeone, A., Acampora, D., Mallamaci, A., Stornaiuolo, A., D'Apice, M.R., Nigro, V., and Boncinelli, E. (1993). A vertebrate gene related to orthodenticle contains a homeodomain of the bicoid class and demarcates anterior neuroectoderm in the gastrulating mouse embryo. EMBO J $12,2735-747$. 
Singh, A., and Tsonis, P.A. (2010). Focus on molecules: Six3--master or apprentice? Exp Eye Res 90, 535-36.

Sjöholm, M., Sinakevitch, I., Ignell, R., Strausfeld, N.J., and Hansson, B.S. (2005). Organization of Kenyon cells in subdivisions of the mushroom bodies of a lepidopteran insect. J Comp Neurol 491, 290-304.

Skeath, J.B. (1999). At the nexus between pattern formation and cell-type specification: the generation of individual neuroblast fates in the Drosophila embryonic central nervous system. BioEssays 21, 922-931.

Skeath, J.B., and Thor, S. (2003). Genetic control of Drosophila nerve cord development. Curr Opin Neurobiol 13, 8 - 15 .

Smith, M.C., Brown, W.R., McEwan, A.R., and Rowley, P.A. (2010). Site-specific recombination by phiC31 integrase and other large serine recombinases. Biochem Soc Trans 38, 388-394.

Snodgrass, R.E. (1935). Principles of insect morphology. Principles of Insect Morphology.

Sobotta, J., and Becher, H. (1964). Atlas der Anatomie des Menschen. 16. Aufl.

Soustelle, L., and Giangrande, A. (2007). Novel gcm-dependent lineages in the postembryonic nervous system of Drosophila melanogaster. Dev Dyn 236, 2101-08.

Spindler, S.R., Ortiz, I., Fung, S., Takashima, S., and Hartenstein, V. (2009). Drosophila cortex and neuropile glia influence secondary axon tract growth, pathfinding, and fasciculation in the developing larval brain. Dev Biol 334, 355-368.

Stollewerk, A., and Simpson, P. (2005). Evolution of early development of the nervous system: a comparison between arthropods. BioEssays 27, 874-883.

Strausfeld, N.J. (2005). The evolution of crustacean and insect optic lobes and the origins of chiasmata. Arthropod Struct Dev 34, 235-256.

Strausfeld, N.J., Sinakevitch, I., Brown, S.M., and Farris, S.M. (2009). Ground plan of the insect Mushroom Body: functional and evolutionary implications. J Comp Neurol 513, 265-291.

Strauss, R. (2002). The Central Complex and the genetic dissection of locomotor behaviour. Curr Opin Neurobiol 12, 633-38.

Technau, G., and Heisenberg, M. (1982). Neural reorganization during metamorphosis of the corpora pedunculata in Drosophila melanogaster. 295, 405.

Technau, G.M., Berger, C., and Urbach, R. (2006). Generation of cell diversity and segmental pattern in the embryonic central nervous system of Drosophila. Dev Dyn 235, 861-69.

Tettamanti, M., Armstrong, J.D., Endo, K., Yang, M.Y., Furukubo-Tokunaga, K., Kaiser, K., and Reichert, H. (1997). Early development of the Drosophila mushroom bodies, brain centres for 
associative learning and memory. Dev Genes Evol 207, 242-252.

Tomancak, P., Beaton, A., Weiszmann, R., Kwan, E., Shu, S., Lewis, S.E., Richards, S., Ashburner, M., Hartenstein, V., and Celniker, S.E. (2002). Systematic determination of patterns of gene expression during Drosophila embryogenesis. Genome Biol 3, 0081-88.

Tomer, R., Denes, A.S., Tessmar-Raible, K., and Arendt, D. (2010). Profiling by image registration reveals common origin of annelid mushroom bodies and vertebrate pallium. Cell 142, 800-09.

Tomoyasu, Y., and Denell, R.E. (2004). Larval RNAi in Tribolium (Coleoptera) for analyzing adult development. Dev Genes Evol 214, 575-78.

Trauner, J., Schinko, J., Lorenzen, M.D., Shippy, T.D., Wimmer, E.A., Beeman, R.W., Klingler, M., Bucher, G., and Brown, S.J. (2009). Large-scale insertional mutagenesis of a coleopteran stored grain pest, the red flour beetle Tribolium castaneum, identifies embryonic lethal mutations and enhancer traps. BMC Biol 7, 73.

Träger, U., and Homberg, U. (2011). Polarization-Sensitive Descending Neurons in the Locust: Connecting the Brain to Thoracic Ganglia. J Neurosci 31, 2238.

Urbach, R., and Technau, G.M. (2003). Early steps in building the insect brain: neuroblast formation and segmental patterning in the developing brain of different insect species. Arthropod Struct Dev 32, 103 - 123.

Urbach, R., and Technau, G.M. (2003). Molecular markers for identified neuroblasts in the developing brain of Drosophila. Development 130, 3621-637.

Urbach, R., and Technau, G.M. (2004). Neuroblast formation and patterning during early brain development in Drosophila. Bioessays 26, 739-751.

Urbach, R., Schnabel, R., and Technau, G.M. (2003). The pattern of neuroblast formation, mitotic domains and proneural gene expression during early brain development in Drosophila. Development 130, 3589.

Urbach, R., Technau, G.M., and Breidbach, O. (2003). Spatial and temporal pattern of neuroblasts, proliferation, and Engrailed expression during early brain development in Tenebrio molitor L. (Coleoptera). Arthropod Struct Dev 32, 125 - 140.

Utting, M., Agricola, H., Sandeman, R., and Sandeman, D. (2000). Central Complex in the brain of crayfish and its possible homology with that of insects. J Comp Neurol 416, 245-261.

Varnam, C.J., Strauss, R., Belle, J.S., and Sokolowski, M.B. (1996). Larval behavior of Drosophila Central Complex mutants: interactions between no bridge, foraging, and Chaser. J Neurogenet 11, 99-115.

Veenstra, J.A., and Hagedorn, H.H. (1993). Sensitive enzyme immunoassay for Manduca 
allatotropin and the existence of an allatotropin-immunoreactive peptide in Periplaneta americana. Archives of Insect Biochemistry and Physiology 23, 99-109.

de Velasco, B., Erclik, T., Shy, D., Sclafani, J., Lipshitz, H., McInnes, R., and Hartenstein, V. (2007). Specification and development of the pars intercerebralis and pars lateralis, neuroendocrine command centers in the Drosophila brain. Dev Biol 302, 309-323.

Voronina, V.A., Kozhemyakina, E.A., O'Kernick, C.M., Kahn, N.D., Wenger, S.L., Linberg, J.V., Schneider, A.S., and Mathers, P.H. (2004). Mutations in the human RAX homeobox gene in a patient with anophthalmia and sclerocornea. Hum Mol Genet 13, 315-322.

Weismann, A. (1864). Die nachembryonale Entwicklung der Musciden nach Beobachtungen an Musca vomitoria und Sarcophaga carnaria

Wegerhoff, R., and Breidbach, O. (1992). Structure and development of the larval Central Complex in a holometabolous insect, the beetle Tenebrio molitor. Cell Tissue Res 268, 341-358.

Wessnitzer, J., and Webb, B. (2006). Multimodal sensory integration in insects towards insect brain control architectures. Bioinspir Biomim 1, 63.

Wheeler, W.M. (1893). A contribution to insect embryology. J Morphol 8, 1-161.

Wheeler, S.R., Carrico, M.L., Wilson, B.A., and Skeath, J.B. (2005). The Tribolium columnar genes reveal conservation and plasticity in neural precursor patterning along the embryonic dorsalventral axis. Dev Biol 279, 491-500.

Wheeler, S.R., Stagg, S.B., and Crews, S.T. (2008). Multiple Notch signaling events control Drosophila CNS midline neurogenesis, gliogenesis and neuronal identity. Development 135, 3071-79.

Williams, J.L., and Boyan, G.S. (2008). Building the Central Complex of the grasshopper Schistocerca gregaria: axons pioneering the $\mathrm{w}, \mathrm{x}, \mathrm{y}, \mathrm{z}$ tracts project onto the primary commissural fascicle of the brain. Arthropod Struct Dev 37, 129-140.

Williams, J.L.D., Guentner, M., and Boyan, G.S. (2005). Building the Central Complex of the grasshopper Schistocerca gregaria: temporal topology organizes the neuroarchitecture of the w, $\mathrm{x}, \mathrm{y}, \mathrm{z}$ tracts. Arthropod Struct Dev 34, 97-110.

Wurst, W., Rossant, J., Prideaux, V., Kownacka, M., Joyner, A., Hill, D.P., Guillemot, F., Gasca, S., Cado, D., and Auerbach, A. (1995). A large-scale gene-trap screen for insertional mutations in developmentally regulated genes in mice. Genetics 139, 889-899.

Yang, M.Y., Armstrong, J.D., Vilinsky, I., Strausfeld, N.J., and Kaiser, K. (1995). Subdivision of the Drosophila mushroom bodies by enhancer-trap expression patterns. Neuron 15, 45-54.

Yannoni, Y.M., and White, K. (1997). Association of the neuron-specific RNA binding domain- 
containing protein ELAV with the coiled body in Drosophila neurons. Chromosoma 105, 332-341.

Young, J.M., and Armstrong, J.D. (2010). Building the Central Complex in Drosophila: The generation and development of distinct neural subsets. J Comp Neurol 518, 1525-541.

Younossi-Hartenstein, A., Green, P., Liaw, G.J., Rudolph, K., Lengyel, J., and Hartenstein, V. (1997). Control of early neurogenesis of the Drosophila brain by the head gap genes tll, otd, ems, and btd. Dev Biol 182, 270-283.

Younossi-Hartenstein, A., Nguyen, B., Shy, D., and Hartenstein, V. (2006). Embryonic origin of the Drosophila brain neuropile. J Comp Neurol 497, 981-998.

Zill, S. (2010). Invertebrate Neurobiology: Brain Control of Insect Walking. Curr Biol 20, R438-440. 


\section{Appendix}

\subsection{5'UTR alignment of Tc-elav with gDNA}

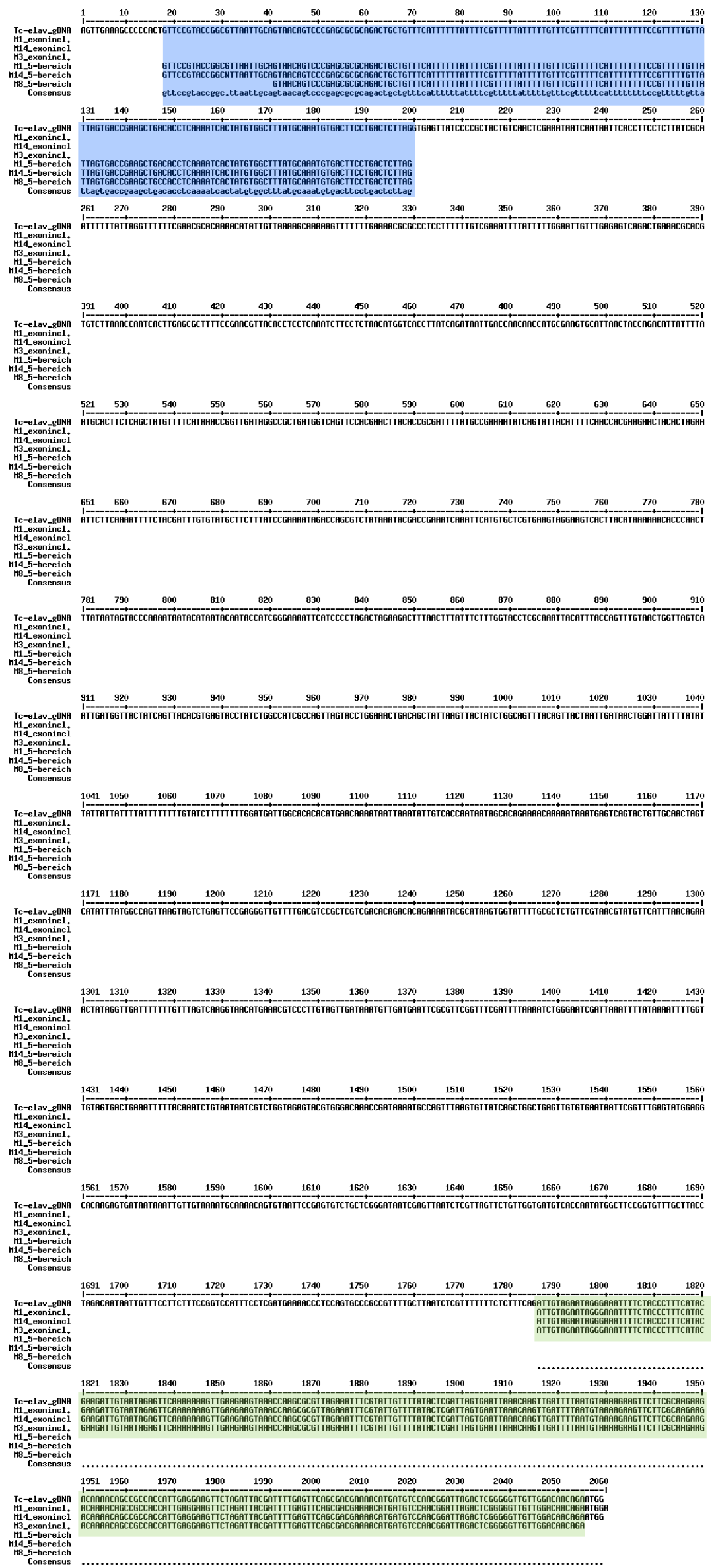

Appendix Figure 7.1: 5'UTR aligment of the RACE PCR of Tc-elav and gDNA.

Different RACE products were aligned. 5'end of the UTR at the top (blue background) and 3 'end of the UTR at the bottom (green background). Translation start site highlighted in red. 


\subsection{Identification of the linker sequence of the $T c$-six 3 overexpression construct}

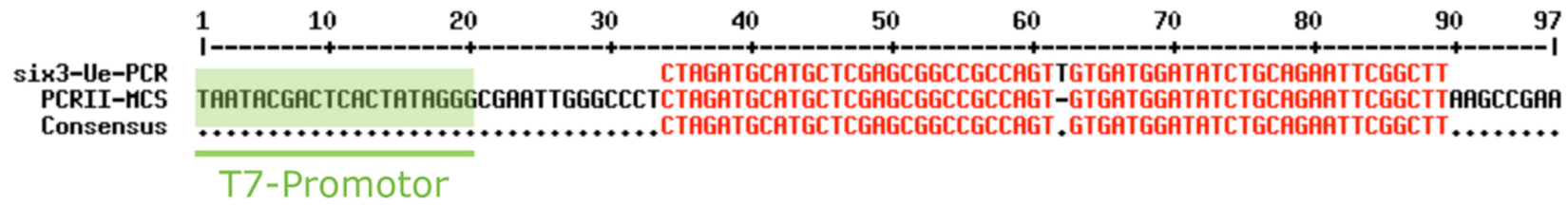

Appendix Figure 7.2: Linker of the HS construct and PCR II Multiple Cloning Site alignment. The upper sequence reflects the linker sequence between the HS-promoter and the ORF of Tc-six 3 of the HS-six3-Overexpression construct. Lower sequence reflects part of PCR II-vector, green highlights the T7-Promoter. The alignments demonstrate, that the linker sequence originates from the PCRII vector (highlighted in red).This part of the PCRII-MCS includes three additional ATGs, which are included also in the HS-six3-overexpression construct (see chapter 3.1.2).

\subsection{Vector maps of the constructs used for embryonic transgenesis}

The following depicts the maps of the constructs, which were used for transgenesis. The backbone vector was $\mathrm{pBac}[3 \mathrm{xP} 3 ;$ Tc-vermillion;SV40polyA] af. Only the sequences which were inserted into the respective backbone at the position of AscI and/or FseI are presented. The color code highlights the respective elements in the vector maps and the sequence. SV40 poly A is depicted in purple. Regulatory regions and the respective promoters are depicted in turquoise. The reporter genes mRFP and DsRedExpress are highlighted in orange, tGFP in green. 


\subsubsection{Vector map of the Tc-elav reporter construct}

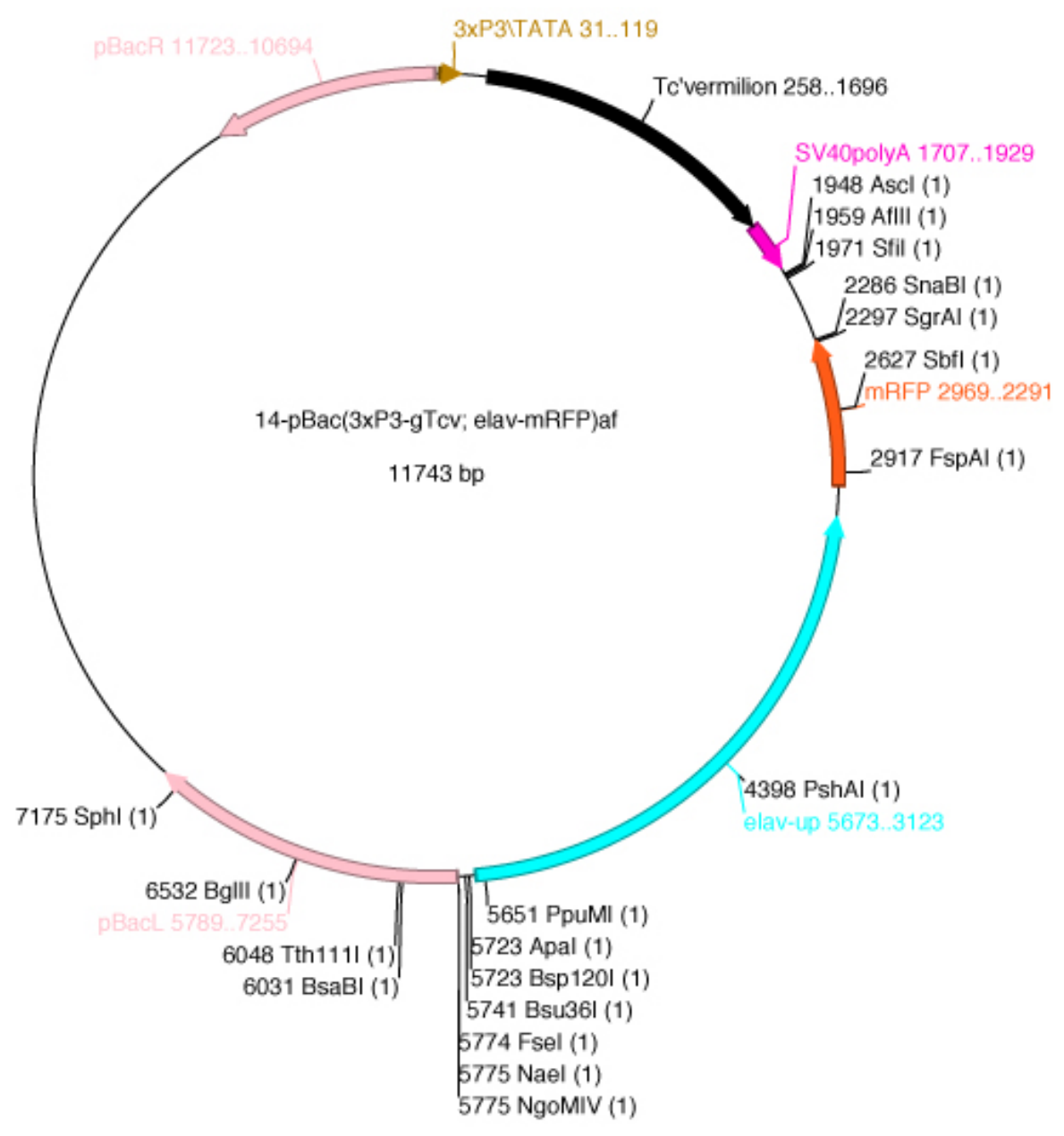

\section{$>$ reg.-Tc-elav::mRFP}

cgcgccaagcttaaggtgcacggcccacgtggccactagtacttctcgagctctgtacatgtccgcggtcgcgacgtacgcgtatcgatggcgecagctgcaggcggecgc catatgcattggegccgeggecgcgaattaaaaaacctcccacacctccccctgaacctgaaacataaaatgaatgcaattgttgttgttaacttgtttattgcagcttataatggtt acaaataaagcaatagcatcacaaatttcacaaataaagcattttttcactgcattctagttgtggtttgtccaaactcatcaatgtatcttatcatgtctggatctacgtattaggcgc cggtggagtggeggecetcggegegctcgtactgttccacgatggtgtagtcctcgttgtgggaggtgatgtccagcttgatgtcggtcttgtaggegecgggcagctgcacg ggcttcttggccatgtaggtggtcttgacctcggegtcgtagtggecgecgtccttcagcttcagcctcatcttgatctcgccettcagggegecgtcetcggggtacatccgctc ggtggaggcctcccagcccatggtcttcttctgcattacggggccgtcggaggggaagttggtgccgcgcagcttcaccttgtagatgaactcgecgtcctgcagggaggag tcctgggtcacggtcaccacgecgccgtcctcgaagttcatcacgegctcccacttgaagccctcggggaaggacagcttcaagtagtcggggatgtcggeggggtgcttca cgtaggccttggagccgtactggaactgaggggacaggatgtcccaggcgaanggcagggggecgcccttggtcaccttcagcttggeggtctgggtgccctcgtanggg cggccctcgecctcgecctcnatctcgaactcgtggecgttcacggagecetccatgcgcaccttgaagcgcatgaactcettgatgacgtcctcggaggaggecatggtgg cgaccggtgcctcgtcgtcgttcaggttgtcettgegcttgctcttgatgcagcccatggcggectcgtcgtcgttcaggttgtccttgcgcttgctcttgatgcagcccatggtgg cttagaggctcgaatctgcagaattcggctttctagaacttcctcaatggtggeggetgtttgtcttcttgcgaagaacttcttttacattaaaatcaacttgtttaattcactaatcgag tataaaacaatacgaaatttctaacgcgettggtttacttcttcaacttttttgaactctattacaatcttcgtatgaaagggtagaaaatttccctattctacaatctgaaagagaaaaa aacgagattaagcaaaacggcgggcactggagggttttcatcgaggaaatggaccggaaagaaggaaacaattattgtctaggtaagcaaacaccggaagccatattggtg acatcaccaacagaactaacgagattaactcgattatcccgagcagacactcggaattacactgtttgcattttacaacaatttattatcactcttgtgcctccatactcaaaccgaa ttattcacacaactcagccagctgataacacttaaactggcattttatcggtttgtcccacgtactctaccagacgattattacagatttgtaaaaatttcagtcactacaaccaaaatt ttataaaatttaatcgattcccagattttaaaatcgaaaccgaacgcgaattcatcaacatttatcaactacaagggacgtttcatgttaccttgactaaacaaaaaaatcaacctata gtttctgttaaatgaacatacgttacgaacagagcgcaaaataccacttatgcgtatttctgtgtctgtgtcgacgagcggacgtcaaaacaaccetcggaactcagactacttaa ctggccataaatatgactagttgcaacagtactgactcatttattttgttttctgtgctattattggtgacaatatttaattattttgttcatgtgtgtgccaatcatccaaaaaaagatac aaaaaaataaaataataataatataaaataatccagttatcaattagtaactgtaaactgccagatagtaacttaatagctgtcagtttccaggtactaactggcgatggccagata ggtactcacgtgtaactgatagtaaccatcaattgactaaccagttacaaactggtaaatgtaatttgcgaggtaccaaagaaataaagttaaagtcttctagtctaggggatgaat tttcccgatggtattgtattatgtattatttgggtactattataaagttgggtgtttttatgtaagtgacttcctacttcacgagcacatgaatttgatttcggtcgtatttatagacgctgg tctatttcggataaagaagcatacacaaatcgtagaaaatttgaagaatttctagtgtagttcttcgtggttgaaaatgtaatactgatattttcggcataaaatcgcggtgtaagttc 
gtggaactgaccatcagcggcetatcaaccggtttatgaaaacatagctgagaagtgcattaaaataatgtctggtagttaatgcacttcgcatggttgttggtcaattatctgataa ggtgaccatgttagaggaagatttgaggaggtgtaacgttcggaaaagcgctcaagtgattggtttaagacacgtgcgtttcagtctgactctcaaacaattccaaaaataaaatt tcgacaaaaaaggagggcgcgttttcaaaaaactttttgcttttaacaatatgttttgtgcgttcgaaaaacctaataaaaaattgcgataagaggaaggtgaattattgattattt cgagttgacagtagcggggataactcacctaagagtcaggaagtcacatttgcataaagccacatagtgattttgaggtgtcagcttcggtcactaataacaaaaacggaaaaa aaatgaaaaacgaaacaaaataaaaacgaaaataaaaatgaaacagcagtctgcgcgctcgggactgttactgcaattaacgccggtacggaacagtgggggctttcaac taggtatcgcgagttgaatgcacatgcgcaattataaatttctattctattttaattttagcaaatttttactaaaactgaaatagaatatttactccaatagcacgaaattgcacgtttc cgattgacttgaatggcgcaaacggcgatttgccgetgcgecgcgccgcactcaccctcgttatcatcggcgataaaaagttaacacaaacttgcaaatgtacgcatccetga tgatagcataaatggcacagcgaaactggtttggtttttgtagcacgtgcggcatatggcaaattaaagagggtaagttggcatctaaaaagagacgecttgaaccacccttga agtaacacacgttgaatatattggatgtagctctatcgcagtctaggtttaataacaagggggaagggtgcacctaatcagtgggtactgaacagctgatggcatacaaagagg ccctttcgecctagtcaaacttattcccagactggaggecgttcaccegcaactctagttttcagagagatttatttagggtccttattaatttttcagcgaagccgaattccagcac actggcggecgttactagtggatccatatatagggeccgggttataattacctcaggtcgacgtcceatggecattcgaattcggccgg

\subsubsection{Vector map of the Tc-repo reporter construct}

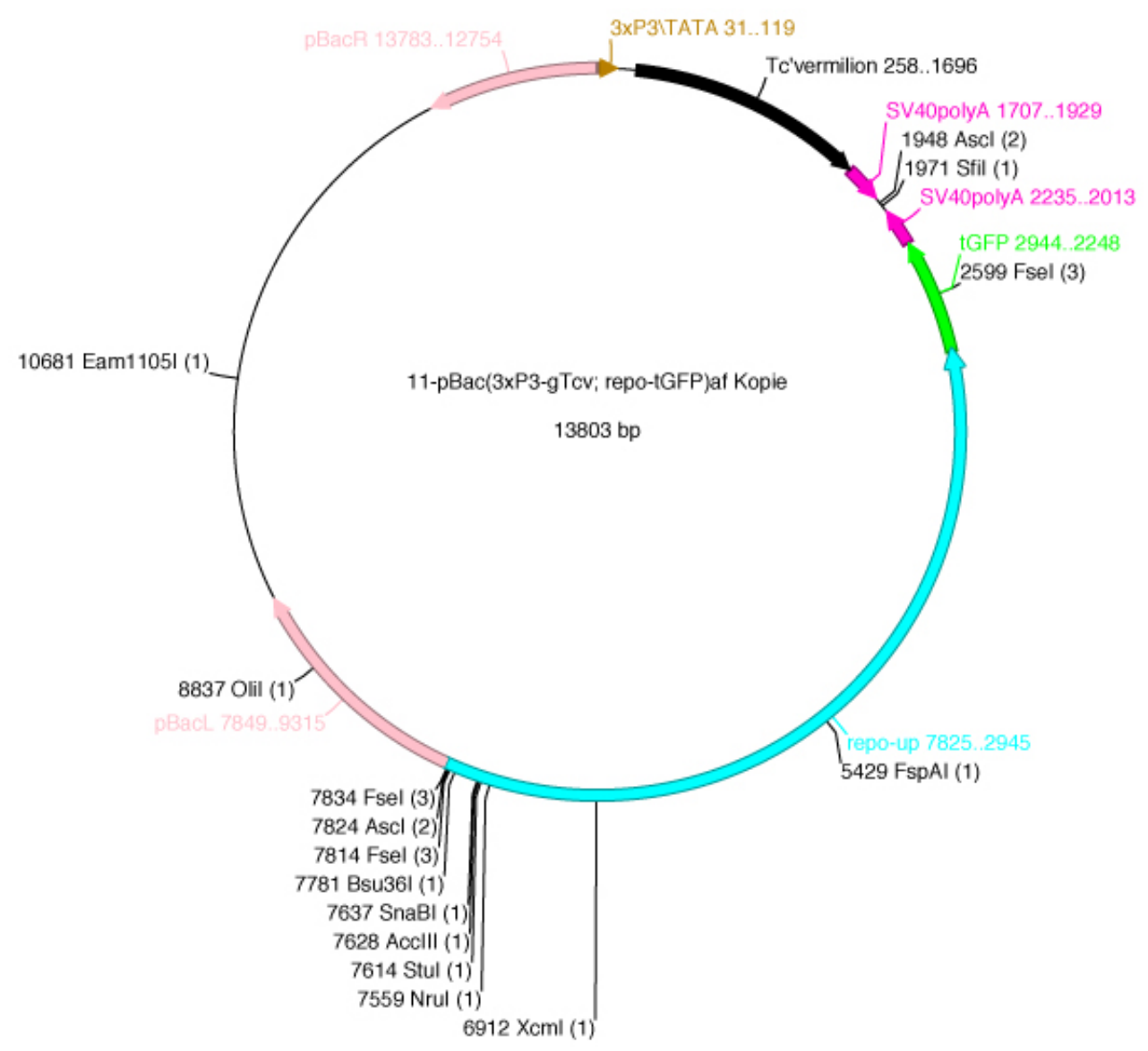

\section{$>$ reg.-Tc-repo::tGFP}

ggcgcgccaagcttaaggtgcacggccacgtggccactagtacttctcgaagcttaagatacatgatgagtttggacaaaccacaactagaatgcagtgaaaaaatgcttta tttgtgaaatttgtgatgctattgetttatttgtaaccattataagctgcaataaacaagttaacaacaacaattgcattcattttatgtttcaggttcagggggaagtgtgggaggttttt aaagcaagtaaaacctctacaaatgtggtatggctgattatgatctagagtcgcggccgctttattcttcaccggcatctgcatccggggtcttgaaggegtgctggtactccacg atgcccagctcggtgttgctgtgatcctcctccacgcggcggaaggcgaacatggggecccettctgcaggatgetggggtggatggcgetcttgaagtgcatgtggetgtc caccacggagctgtagtagccgccgtcgcgcaggctgaaggtgcgggtgaagctgccatccagatcgttatcgcccatggggtgcaggtgctccacggtggcgttgctgcg gatgatcttgtcggtgaagatcacgctgtcctcggggaagccggtgcccatcaccttgaagtcgccgatcacgcggccggectcgtagcggtagctgaagctcacgtgcagc acgccgccgtcctcgtacttctcgatgcgggtgttggtgtagccgccgttgttgatggcgtgcaggaaggggttctcgtagccgctggggtaggtgccgaagtggtagaagcc gtagcccatcacgtggctcagcaggtaggggctgaaggtcagggcgectttggtgctcttcatcttgttggtcatgeggecetgctcgggggtgccetctccgccgcccacca gctcgaactccacgccgttcagggtgccggtgatgcggcactcgatctccatggcgggcaggccgctctcgtcgctctccatggtggcgaccggtaccactttgaattcacta gtcgagctcggattggcaattgcttaatgaaccaaattggctacacagttctggacaaactgacttctgacattattactttatccaatttatatttaacgagctgaattaaggacgctt ttggcggccaatccgggggttcgtcgcaacgccattggtgtggaaggaggagtcgagggaccaatacgggcgatgcggtcacgtggacaaggcccgatagggctgcttgt gggaaaatcgggaggattcgggatattcaggaggactggatggtcttgacgcgactgaaaggggtcttgaagcaactgtgcttaaaagactaggattccaaagagccacaca 
tgattggttcaaaaattgcaagacgtgccttgtttatactttttttttatactaaaatggttataaaactctccaaatttaactatatctaaaatctttgttataaaaaatgatgaaaaaaaa cataaataaaacaactttggtcgtgtcttttctcctaaattaatttttctttatatatttatttggatgtggaatgtgggtagtttaagatatttatccacattaatctgttttttacagagggag ttattgttcattttttgtccaatttggatttgaaatattgatttttagatttgttttattggaaaatctattaaacttataggtatttgatagatcgtttctattgaatcattttttttatatctatttagg taaacatttctgaaagctgaatttataacattgtttattatctggttttaaattaaaattacttttgattctggctcaaaaaatgtgctaaattgttaaaaattggaagaaatagtttactttta acctgttgcaattaaattttatgaagagaactttttatctttatttttaaatgaatccttttttggetgtattttcttaattatttgtgetggettgttttggcaaaatctcttaattttagtaatttcat gattcaaaaccaactaaatcacttgttatcttgacaaaaatgtcataatttgggtccaaaactacgattttctgacaaaactaactcattttatttggtcttttccatcatgattttttaact tggtgagtaactgtctgaaaaatggtcagttacatcagtctgaactttgactgaaatttcataattaatttttgttggettgttttggtaaaacttcttaaactatgtaatttctcggctcaaa aaccaactaaatcacttgttatattgacaaaaatatcataatttgggttcaaaactacgattttctgataaaaataacttaaaacacgatagaatcgettgaaatttgctgacctaaatc gaagattttttttgtttttcgcgttgaaatgagtctgtggctcaaaagtaagttaattaataattctttgcaattttggctgattttataattagcataaaggttaaaagaatgaatttttcgg gcttttttggcacaaaagcaaactaaatcactcgaaaacttgactaaaataacattatttacgtctacaatgattattttttacaagttttttcaaaatttcattgcagtgactgaataaatt gettggaattcaaacttaaaacgatgattttatttgatatattcgcttattattttttaatttggtgagtaactgacagaaaaatggtcacttatatcgttcagaattttgactgaaattttata attgattttttgttggtttattttggcaaaacctcttaaactatgtaatttctcggctcaaaaactaactaaatcacttgttatctcgtctaaaataccataatttggtcttttccttaatttttttt gatttggtgtgtctttggcgetgaaaaagtaaattacatcattcgcaactttggctgaaatttcataattgatcttgttgctagcttttttacaaaatcgtttgaactcagtaatttttaa gcaacaaggcaacaaatcagtcgaaatcttgactcaaatcaacgattttttggtcagtttttataaaattttagattaaattactcaaacataatgtaaattaccaggaaataacaacct atatctaaaacaatgagtttctggtctggtcattaatttttttaatttaaaagatgaacttaatgacacggaatagcacaatttgggctcacgatgattttttgtctattattacaaaatttc ttgtagtttgtgtatttctctggacatgtcaaattaattgcttttagctggcgctgacaatatttgttaagaaagaagtgaaaatttgagataaagcaaaaatattttgatatatttttaaatt cttagaataattaatattaataaacctgagacagtcaaaaacgaaaaagatggagagacagacaactgaaaaggattttgattcagcaacagtcatattatgatgtcaaatagtgc gcacatgattggctgatttttttatgaaatcaggatattatttttatttatttatttctatttggatgtcaagtaaaattgacaaaagatcagatttttgtatttttatgtaggtatgcaagtacat acccaatgttaattttaaactttgagtaataataaaaaatcattaccaaaagttaagcagtgtttctctttgtttccttttccttgttctgcatattattattatattatattatattattctgectt atatcatctaatattaatgttaattaagtaatcacagaccactttgatcagaaatttaattgaaaggtagttagtcacagacatacagtagaaagcgtaagctttttcacgttaaaagaa aaaagcaaaatcttgtacgacaccgccttaattataccaaaaaaataaggtcgcctgtacataagcttaatttttttggcgtcaaaccaaattaatatcaaatttatttttaataaattgt gccttgttctataattgggttctataaattctcagatttaaaattaaaaattgtcaagaccagagaacaaaaaaataggattctaaacaaagttttggtcttcttagtgttctaatttttca taatctggccaagttaagaaaaatttttggtcaaaaaaactgattttttttaaatttttgtgcatgtgcttgtaaaaaccgcgcattttcgttgtgcattttaagagtgtaaggcttaa gcgttaagcgcacgcatttgcctcaatcacccataagtgaatttatcaatttacccaatttttgcctattagcgacaatagctattagtgcagctattagtatattagtttaacagccca gattccttaatgcaatccaactcaatattgtccaattgttgctattaaaacatttcacacagtaaatgttcaccagtccaatggagactcttattcgagagggttgtcttggccggagg gtgccettacgctagtgtatacagtgatacgaaagtcgaaccagtgcacaagcaagtgcatcaaacgtcatcaaaaaccgattcaacaagtttcgtcgtcgtcgtcgcatcatca aggattgtgtgaaacgacgcaagtgttagaagtggattttggtttcgccgattgttttgtttaaattaaatcgagtggaaataattaaggaaaaatgtgtgtgatattttcgatggaatc ttgtgttgcagacacttgttgcgaggctgtatcatctcgtcaattgcactcaattaaggatccattagatttaatttactttaactcgctcaattgctggtggcgtaggggtcacatgta ccgacagtgtcatacatgcgggacacgcaaccgttgggatagttcaaacaaaaataataggaattatgggagaggaaatgcccacacttgatcgaattcgagcgtacagtcg ccggaaaatttcggtctggacacaagtgcccaaaatttaattggaaattaaaaaacgattaaaagggtcgaacaaatgtaaaaaagagcaaatatattttgtattttttcgccgca taatttttgatgtcattgaaaatttttttttatttatttttggtttttgaaattgttcttcttaagtaataaaaatttgataataaaattagtgtttttttaaactatgtacgttagttcaaaattttgg tttattattacaaacagaacaagcatatttgtaattttttaatacagtttgagatagtactgtgctgattttaactgcagtatttgttttatagtccattgtggtttgattgcttcaccgtgattt cacaaaggactgacttcgtgaatataaatgcgagtgcgttgtgtaaaaagtctcgtatttaaactcatttagactgctttttcttttatttttttaattaattttaaataatttttggctaattca aagtaataaaaaataattaggtgatagagaaactggaactaacgtaaaatgtaatggaaaaaattcaaaaacccaatgaaacactgctgagacgttacatattgcaaagaaag ccgaattccagcacactggcggecgttactagtggatccgagctctgtacatgtccgcggtcgcgacgtacgegtatcgatggcgccagctgcaggcggccgecatatgcat cctaggectattaatattccggagtatacgtagccggctaacgttaacaaccggtacctctagaactatagctagcatgcgcaaatttaaagcgetgatatcgatcgcgcgcaga tctgtcatgatgatcattgcaattggatccatatatagggecegggttataattacctcaggtcgacgtcceatggccattcgaattcggceggectaggcgegcc 


\subsubsection{Vector map of the 'Tc-six 310 up' construct $^{\prime}$}

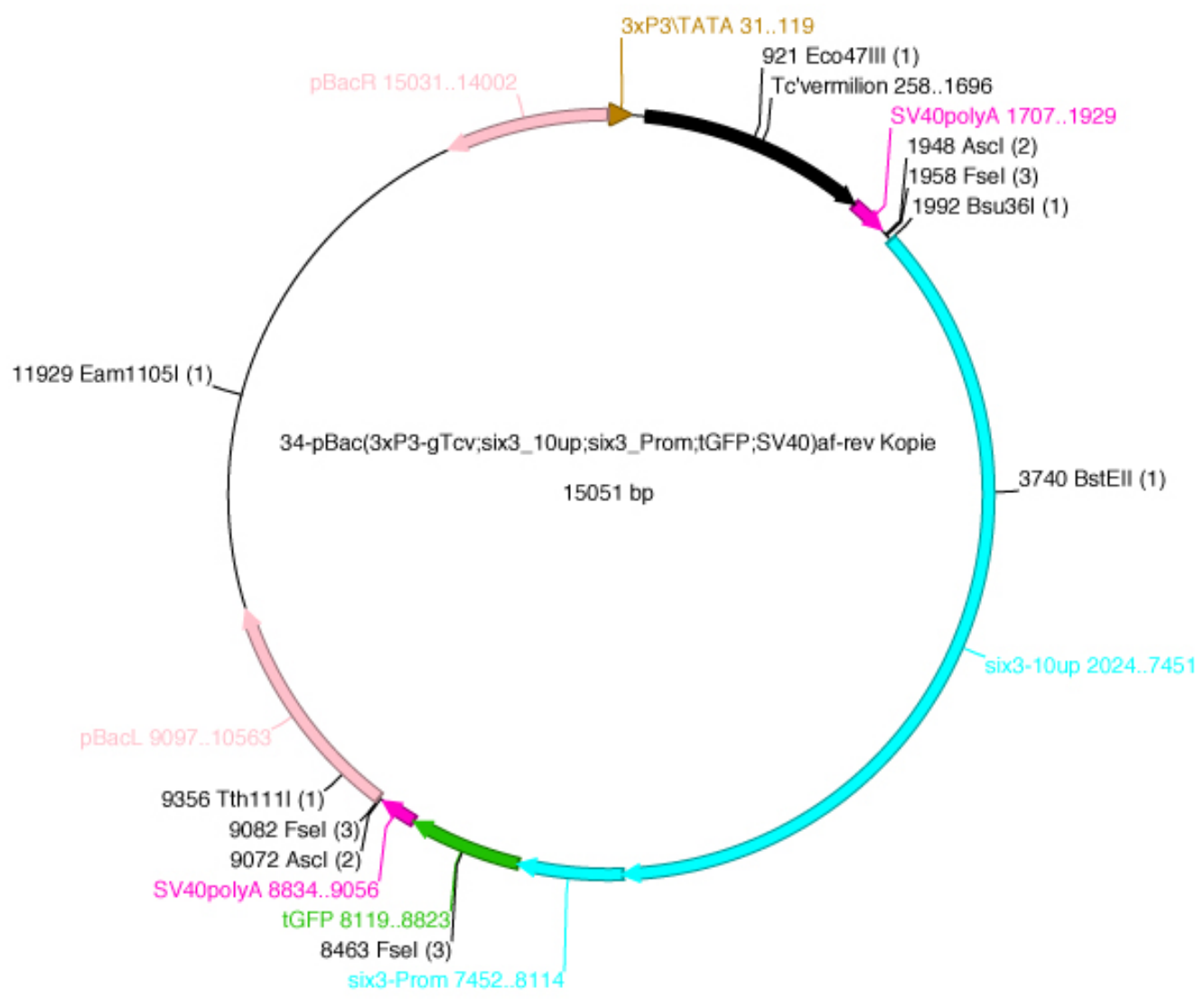

> Tc-six3 10up::tGFP

ggcgcgcctaggccggccgaattcgaatggecatgggacgtcgacctgaggtaattataaccegggecctatatatggatccTCCATGCCCATGGTGAATG GAGCACATGGAGCCCCGGTGTCAATCATCTCCGTAAATCCGCTTTCTCTATTGTGGTTTGTCGGATTAGTCC GGCACTCCCTCCTCAAATCGAAGCTAAAATATGTAGCGTGTACGGACCTTATGCACCGTTGTTTGCATGTTT GTGTTGCAAAGCTCCTTAGTGATGCGATTAAAAAGGTACAGAACGGAAACACGTCCCTAATCCTCTTGGAT TTCCCCCCGATTTTGGAAAAAATCACTCTTGGTCGATTTTGAAGACGAGTAGCGTGTTGTAACCGAATTCGC CAACCCTATACCGAATAAAAGCCGAACGAGCGAGCAACAGGGTATAAAATCGATATTATTCGCATCAAAGAT CAAATTTAGCCTGTAATCGGCCACCTTATTAGCAGGCGGCTCATTTTCCATGGCTTTCTCTAATTTCAAGCGC GCCTGTCTGAGCGCATCGGCCCCCGCGTACGATACTCCTTTGTTACCGAATTATCGCAGGTAACTTGATAGC CAGATTGCAACATTTGGGTTACAAACAAAGTGCAGTCAGAGATGCGTTCGTAATTTTTCTGGCAATTTTGTT TACTGTTTTATTAATATCCAAATCGGGGGATCCGATTTCCAAACCAGAAAAAAGGGACCGCACGGATCGCG GATGCCACGGAACCACCGGACGAGATATAGACACTTACGTAACAAAAAAAATACGCGAAACAGGTGGGCA GTCTGCCAGGTACACCGATTTTTGAAAACAGAAACCAGAGCAAAAAGTGCCCAGGACGGGCTAAGCGGT GGCTGCTCCAAAAATGTTAAATAAGCTAAATTGAACAAATATTAAAAAACCACATTTGTAGCAACTTTCGTT AATTGCATGATTTTAATATTTTTTGTCTTAAAAAATAACTAGTTTTCTTTCTTTTTAACCATTTGGGGACAATT TTTTTGTTTTTCTGGACGTTATAATTTGATTTTAATTTTTAATTTTAATTTTTTTTGGTCTATTTTTAATTTTAAC TGATGAGCATTCATTTTTGAACACATTGTGTTTATTCTTTGCTATCGTTCGGACGTGGTGTTGTCATCTCTGG ATAAGTGTGAACCGTGATGTGGCTTTAATTTCTTACCAGTGCCCATTTTTACGTAAGTTGTAGGATGTCTCAT AGTTGAAGATCTTGTGGCTTCCATATTTGAAATCATAGCAGAGTGTGAATAGAATTGACAATTAAACTCTTG GCCGCAATCACATAAATAGAGGAAACCGTTGACAGTAGTGGTAACACCAATAAGCCATTTAAGTGTCTTGG GTGTCAAGGGTCGTCCGTTTGTGTTTTAAATCAGTCACAAATAATGGGGTGCCGAGGTCATGTGTGGTTTCA GAATCAGTGAATGTAATACCGCAAACATTTAATTTTACTGCGTCGTGTTACTTCTCGGTGTATAGTTATCGGT TATCACTTGAAACCGCGTAATTATCCCGATTTTTGCAAAAAAGTTCCGCTTTACGAGACCCAGATATTCCCC AGTCTCATTTATCGAAACAATGAAAACGTAAATAACGAGCCAACAAAAGCTCGTCGGGGATTCAAAATGAA ATCAAATCTGTGCCGAGGAAAATAAGTGTAAGAGATTGACGTCGGATGAAAATTGAAAAAGGAAATTTCTT TTTAGACTCAATTGTTTCGCTCGGCTGGGCCGTACGGTCACCGGACAGTGTATAGCCAGACTTGATTAGTAA 
GAATGCTTTTTGTCTCCTGTTCGCCTCGTTTGAATGTTGACGCTGGAACGAAGAGGGAATGCAGGGATGAT GTAGGGGAAGTCGACCCGTGATGGAGTGCATACAACGAGATTATGGCACAAACCCTTGTATCTCCACGACT GCGATGCAAACTAAACTCCAGGGACTTGTGTTATTCGATAACCTGGATGGGAACCCAGTGGGAATCGCCCG CTTCTTGCTACTTTCCACAACTGCACAAACACAACGCAAAAATAACGTAGAAAAAAAAAACGTTAAAAAA ATCACAGAAATAACGTTATTTGTAACGAAAACATTACCGTTGCTTTTTAGTAACTATTTAAACGATTTTGTAA CGCAAGAATATAACGTATTATAGAAAATATAAAACCAGTAGTTAAAATCAACAAGCAACGATTTAAAAAGGC TGTAATTCAGTTATTTGCAACATTAATTATACGTTTAAAATAAACGTAAGTAAATACGTTTTCTAAAAACGTTA AAATTTTATAAATTAAAAATTGGTGAAAAAAATAAAGATGGTTTTTAAAATACGTAACATAAACGTTAAAAT ATAACGTAATCATACAACCAAATAACAACGTTGTAAAAACGCGTACTGGAATTTTGCTTTCGTGGAAAATTC AGTAGTCAAAAAATTGGTGGTTGATTGAAAATGATGGCAATAAGGAGGCTCCGGGTCCGTTTGCTCGTCGG CAATCCAAACCTAATATCCCGCAAAGAGGAACAAACAGCACAATAGGTCATAAATGAAGCGTAGGAGGCTA TCGTTGTGCCTTGGGCGATTATATCGACTGTCAAGTGTTGACAGTTGAAGCACGGCGGGAGTCGGATTAGA CCGGTCGTAGAGGGCTTGAGTTTCACGCACGGCCGTCTAGTATCAACATATGTGCCCAAACCTAGTCTTTTA TGTGAATTATTAACGTTCCGATTCGTTTCAAAAGCAACAACACTCGAATGTTGCTCCTCGCTAGTGAGATAC AGAGAGCAGCTTTTTCATTTATTATTGAGCGGAGATTTTGCTGCAGGATCCTGTGGGAGTCGGGTTGGGGG ATGTAATTCGCCACCGACGACCACCATTAGGAACATCAAGTATGCAGGCGCGGTAAGACGGCTTATAAGCA CCTGTGCGCAGGATTAGAAGTAATAAGGGCTAATGACCAAATGTACGTGACGGGGAGCTGGCCGCGGGGAT CTATTCACAAAGTCGGGAAGAAAATCTCACTTTCTTAACTCTTTTTTCAAATAAGATGACAACCCTTCCGTT GAAACTTAATTGGGGTGAATGTTGTATTTTTAAAAATTTATTTAGAAAAAATTCTACTAAATCGTGTGCAACA TTTTTTTGCATTTTCTTCAGAAACTTTAAGACTTAGAGCAAAAACATCAGATTGCGCTTAAAAATATCTATAT TCTTGTCAAAACCCAGAGCCGCTCCGAGCCATGGTTCCGTCTACTGAGGCATTTTAATGTTTTTTTACTAAA AAAATTATATTTGAAAAACTAAAAAACTAAACTGAAAAAAATTCTAGCAAACTTTTATAGACTTTTCTATGC TTTTACAACCATCTCGAGTTGTTAAAAGTACAATACAAGTCATAAGGTTTATTTTTTCAATGTTTAATTTTTCC AATTTCTGCGAAATTTTTTTCGATTTTTGGTACCAGGAAAATTTATCGAGCAATAACTCCGAAAGTTTTAAA GATAACGCTTATCTTCAAAAAAGATCATTATTCTCCGACTAATAGTTTTCAAGAAAATTGCAGATGAATGCA AAAATTTGTAAAATTTTAAAAAATTCATAACTGAAGAAATTATTAAGAATTTGGCGATTTTCTCAACGCTAAT CCATTCCCCAGATTATTTTACATAAGTAAGAGTCAAAAAAAGTTTACTTTTTTGAATAGTATTGCCATAATTTA TTTTACTTTTAATTTGAAAAATGGTGGATGCGCCGAGTTATTTTTCAAAAATTCATAACTCAGAAACTAAAA GTCGTAGAGCAATGCAGTTTGTTTCATTAAATTCAGGGGCTCATTTTCTGTATTATACCAACTTTTAACAAAA TCTAACATTTTAAACTTTATTGCAAAAAACTGAGATATGCATTTCCGATAGTGGAAAATTTTATTCAACAAAA AAAATTCATAACTCAAAAACTAAAAGTCGTGGAGCAATGCGGTTTGTTCCATTGAATTCAGCGGCTCATTTT CTGTATTATCCTAATTTTTCACGAGATTTAAAATTTTCAATTTTTTTGCTCAAATTTCCTGAGTAAAACAATTT ACCCTTCAAAGAGGTGAAAAAAAATATTTTTTTTAAACTAACTTCTGCAAATTTTTATGGACTTCTACATGTC TTAACAACCCACTAAAGTTGTTAAAAGTCCACAAAAAGTCCAAATGTTCGATTTTTTGATGTTTGATTTTTT CAATTTTCGTCGCAGTTTTTGTCGGTTTTGGGTACCCGGAACATTTCTCGAGCAATAGCTCCGGAACTATTA AAGATAACCCTATAAAGTGTACTATCGTTGGAATGCTCTTTAAATTATCTATTTTTTTCTAAAAAGATTATTATT CTCCGACTAATAGTTTTCGAGCAAATTGCTGCTAAATGCAAAAATTGGTAAAATTATAAAAAATTCATAACTA AAAAACTATTGGGAATGTGGCAATTTTCTCGATGCCAATCGATTCCCCGGATCATTTTGCATAGGTGTGGATT AAAACAGTTCCACTTTTTAGAACAGTTTAGCCGTAAATGAGAAAATAAAAAAAAATAAAAAAAACTTACAC TTAGTACCTTCAAAGAGGTGAAAAAAATTTTTTTTTAAACTCACTCCAGTAAATTTTTATAGACTTCTACATG TCTTAACAACCCACTAAAGTGATTAAAAGTTCACAAAAAGTCCAAATGTTCGATTTTTTGATGTTTGATTTT TTCAATTTTCGTCGCAGTTTTTGTCGGTTTTGGGTACCCGGAACATTTCTCGAGCAATAGCTCCGGAACTATT AGAGATAACTCCATAAAGTATACTATCGTTGGAAAGCTCTTTAAATTATCTATTTTTTTCAAAAAAGACTATT ATTCTCCGACTAATAGTTTTCGAGCAAATTGCTGCTAAATGCAAAAATTGGTAAAATTTTAAAAAATTCATAA CTAAAAAACTATTGGGAATTTGGCAATTTTCTCAATGCCAATCGATTCCTCGGATTATTTTGCATAGGCGTGA ATTAAAACAGTTCTACTTTCTAGAATAGTTTAGCCGTAAATGAGAAAATAAAAAAAATTAGAATTATGCAGC AATCACTCTGGACTATTAGAGATAACACTATGGGGATTATTAATTATTATCGTTAGAATGCTCTTTTAATTATCT ATCTCTTTAAAAAAACATTGTTCTAAGACTAATTCTTTTCGTAAAAATAGCAAAATTAAATAAAATAAAAAA ATCTTAAGTCGAATTTTTATTTTCGCTTCGTGTATCAAATTGATTTCGTTCCCACACAAGAATATAATAACTGT TAGGTGAAGTAGGGGTTAATGTGCACTAAATTCGGTCTAGATGTGAAATATATTTCCACATTTAAATGTGGGG TGTGACGTCCCGATTTGTCGTATGATAATAGCGTTGTCGGCACTTGTCAGagatctGTGTACACCGGTCCTAAA GGTTTGAAATGAAATTTAAATAGGTAGATAAGGTGATATTTTTTTGTCATTTATTTATTTGCATGCCTACCACC TTTGTACTAGTATGTAGCTAAAGACATTGTGCGTTTCGATTGGGACATTTATGATCATGTGATACATGGAATA AATTACGTACCTATACCTTCCATTGAGAAAAATTGTACTTTTTTTTCCACTTGAATTATAATAAAGACTTAAA AGTTTCAATAACATGGTTGTTTTTCTAAACCTTGAGCTTTCATCCACACCCCGTGTCTCCTTCGTAAACCCAC 
CATTACCCATTTCTTCCCGAAAAGCGATCCATCCTCGTCCATAAAAAGGCGTATCTCGTCCGCGATTCCGTC GACATCATCTATAAGAGAACACCACTTTATCCATCACTTCTCGTTATCCGGCACAAAGCCTCCACAAGTCCA CACTTTTGAGTGACACCGACTCCTCCCATCGCTCCTCCACTCCCCTCCAATTTCGCTCCCATTGGCCTGGTT GTAACCAAGTAACCAGAGTCCCATCCCCGTCCGCGCCAATGGTCGCCTCGGCAGTCTCGCCTCAGTGTAAC CGCGGCCTCCACCTGGCAAGGTGCGTGTGATGGTGCACTAGCCCAAGCTGGCAACCCATggtaccggtcgecaccat ggagagcgacgagagcggcctgccegccatggagatcgagtgccgcatcaccggcaccctgaacggcgtggagttcgagctggtgggeggeggagagggcacccccg agcagggecgcatgaccaacaagatgaagagcaccaaaggcgecetgaccttcagccctacctgctgagccacgtgatgggetacggcttctaccacttcggcacctacc ccagcggctacgagaacccettcctgcacgccatcaacaacggcggctacaccaacacccgcatcgagaagtacgaggacggcggegtgctgcacgtgagcttcagctac cgctacgaggecggccgegtgatcggcgacttcaaggtgatgggcaccggcttcccegaggacagegtgatcttcaccgacaagatcatccgcagcaacgecaccgtgga gcacctgcaccccatgggegataacgatctggatggcagcttcaccegcacttcagcetgcgegacggeggctactacagctcegtggtggacagccacatgcacttcaa gagcgecatccaccccagcatcetgcagaacgggggecccatgttcgecttcegccgcgtggaggaggatcacagcaacaccgagctgggcatcgtggagtaccagcac gecttcaagaccceggatgcagatgccggtgaagaataaagcggccgcgactctagatcataatcagccataccacatttgtagaggttttacttgctttaaaaacctcccaca cttcccctgaacctgaaacataaaatgaatgcaattgttgttgttaacttgtttattgcagcttataatggttacaaataaagcaatagcatcacaaatttcacaaataaagcatttttt cactgcattctagttgtggtttgtccaaactcatcatgtatcttaagcttggegegcc

\subsubsection{Vector map of the `Tc-six3 5up’ construct}

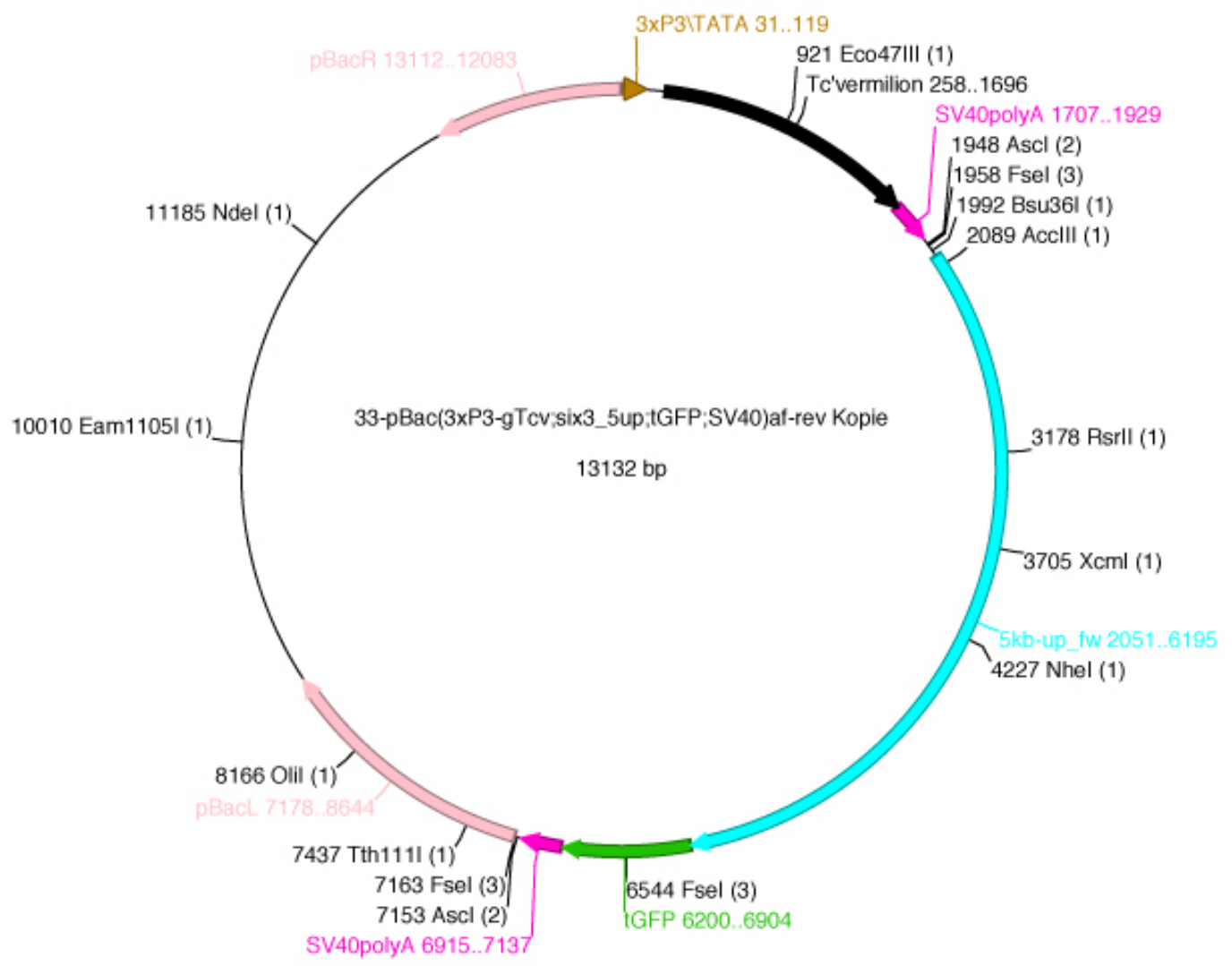

$>$ Tc-six3 5up::tGFP

ggcgegcctaggccggccgaattcgaatggccatgggacgtcgacctgaggtaattataaccegggccctatatatggatccaattgcaatgatcatcatgacagatctTTG GGcACCCGGAACATTTCTCGAGCAATAGCTCCGGAACTATTAGAGATAACTCCATAAAGTATACTATCGTTGG AAAGCTCTTTAAATTATCTATTTTTTTCAAAAAAGACTATTATTCTCCGACTAATAGTTTTCGAGCAAATTGCT GCTAAATGCAAAAATTGGTAAAATTTTAAAAAATTCATAACTAAAAAACTATTGGGAATTTGGCAATTTTCT CAATGCCAATCGATTCCTCGGATTATTTTGCATAGGCGTGAATTAAAACAGTTCTACTTTCTAGAATAGTTTA GCCGTAAATGAGAAAATAAAAAAAATTAGAATTATGCAGCAATCACTCTGGACTATTAGAGATAACACTATG GGGATTATTAATTATTATCGTTAGAATGCTCTTTTAATTATCTATCTCTTTAAAAAAACATTGTTCTAAGACTAA TTCTTTTCGTAAAAATAGCAAAATTAAATAAAATAAAAAAATCTTAAGTCGAATTTTTATTTTCGCTTCGTGT ATCAAATTGATTTCGTTCCCACACAAGAATATAATAACTGTTAGGTGAAGTAGGGGTTAATGTGCACTAAATT CGGTCTAGATGTGAAATATATTTCCACATTTAAATGTGGGGTGTGACGTCCCGATTTGTCGTATGATAATAGC 
GTTGTCGGCACTTGTCAGTCGACTTGGGACAGTTTAGGTGAGGGCGCAAGTGAAGCCTTGCCACGAGGGA GATCCGGAGACAATTTTCCGGTTGGAGGAGCGTCCCGTGGGGGAAATTACAATATTTATGATGTCATTTAGA TCGTCGATCGTCGCTTTGTCAGAATTCAGAGCTCGTTGCGACTATTGTGAGACGCTTTAAACTCCATCAATA ATGTATCGCAGCAATTAGAGCTCATTCAATCAGACGAACGGGTGATCCTGCCACGTGTACGGTCTTATACTC CTAGAGATGACTCCGTAATCAACGCGAAATTACACCTCCAACTCGGGGAATTCGTTGAACTTTCTCAGCAG ATATTGCTTGCAAATTACATCACGTTTTTAACCATTTTTGTCTAAGTAAAAGGACGAGTACCCAAGTTTTTTA ATGAGTTTGGGTTGTTTGTGTCTACCTCGGCGGTCCGTAATCTCCCTAATAGCCTTAACTGGAAATATTAAAA CAAATAAAAGCTCTTGACGTGGATCGAGACGGAGCTGTGATCCGGAGTTTGTTTAGATAGACAGGGCGATG GCGCCAACCACCGCTATAACAAAAATGAGAAAAACGCAAGTGTCTCATGTCACAGTCACGTCCCTTTGTGC ATCTTCATTAGACGCTGTTTCGACAGTTTTATTTTTAGGACACTTAAAAGGTGATTTCTATTAAGCGGTAATT GCTTTGTGTTCGACTTGGAGTGTTTCTGCTCTGCAATTATGTCTTACGTTTAATTATTTTGTTTCCAAATATTG ATACGGGCGAAGAATTTCAAAACTTGTTTTTTCGGCGATTTGCTGACAAAGATATACATGGGTTTTATTATCG AAAATACTTTTTTAATTGATCTGAACCACTTTACGCCGAATGCCATTAAATAACTATTTGAGCGGCAAATGCA CCGGTCAAGGTGATGGCGGCATCGCTTTAACGAAAATGGACTGGAATTGCCAGAGGAATTGTGGAATTATC AACGATGACACATTTTCAAAAATTTTAAGTGCAAAAAGCCCAACAGCCAAATTATTTTTAATTGAACGGAA GAACAAGCAAAACGAAGCAACTCGTTTCATTCATTTAAAAAATGTATGATTACAAGAAAGTGTTTTATGGA GTGATTCCACATTGTCTCGTTTATTTCTGAAATTTCCTAATTGCAACCAAAAATCCTACTTAGGTAAACTTAC CACTTTATGCCACATTTAATATTTAAATAATTTAATTAAATCAAAATTGGTTCGTTCTATCTTTTAAACATTATT ACGTAGTTCATAATTAAATTTTTAAAACAAAAAAATCTCACTCATAAATCAATTTTGAATTAATTTTGCTATTT CTAACAATTACGGATCTGAACAGTTACAATTAGCATATTATTGGATATCTACATCTAAGACAATTTCAGAAAT AACCCATATCTAGGTTAATTGATTCACTCTGTTGTTAGAATTAAATCAATTTAATTGAAAAGTGCTAGCAAAA TAAGCCTTTAAATGAGGCAAAAAAATTATTTAACTGTCCTTTTTCAATATGAAAAATTTATGGTCTCTTGAAT AAAATAATTCATAATTTATTTTTGTTCTCAAAAAATCGTTATAGGTTTCTGCGATTTTGGCTGAGGCTGAAAG TTGAAAAATAAAACATGTGTTAAAATTACATTTTTGGCTATTAGGTATTGGCTATAATTTTAGATTCTTAAAAA TTACTTGAATAATTAATCTTCGATAATAATTTGAATTTCGTGCTAAGCTGAAATGTTATATTTGTTGTTAGCCA AAAATCTGCAGCTTAGATGAAAAATTACATAGGGAGCACACTTGAAATACAGGGTGAGCCTTTTGAATGTA GGTATATTTTTAACATATAATCTGTCTCAAAAACCAATAAAAATACAGAAATCATCAGAAGGGTGGTATCATT TAATTTAATAACAATCAATTTAATTAATAATCAATTAATTTCTAGTGTTCAATAAAAAAATTTACGCGATAAAG CATTATTTATAAACATCGTTTGTTTAACAACGATATTAGATGATTTAATAATAATTAATTAATATTTATTATACAA AATTAACATCGATAGAGAAAAACTTCATTATTACTTAATATTTGACGCTGATTTATGTGTGAATGATTGTATAA TGTGTGTTTTTCATCTAGTCGTCTGTGAATTTCTCATGTAAAATCAGAAATTCCGCTTTATTGAACTAATGAC AGGCATTTAGACACCGAATATTACTATTTTCCATTTATTGAGTCTCCATTTCTGAAATAAACTTCAATAATAAG TGTTTTCTTGCACAGTGTAACAATTTAGTTGTATTTTAACGAACTTTTTAAGATTAATTAATTGACAATTTGTG AATTTCACAAGTGACAGTTTTGAGCAGAGCACATATTTTTACATGTAAACCAATTTCAAAGTTAACTAGATG ACAATCATTTAAAAACACATTGTATTAGTAGGTATTTCAAAGTAAGTATACAGTGAAAGCCAAAGTAAGTCA AGTAAACTGAAAATAAAGCATTTAAAAAAATTTTATACCTAGTACAAAAATATAGCAAAACTTGCAATGGTC AATAACAGAGTCGTTAGTGCAGAACTTAGGTATATTAATTGGCCTTTAAAAAATTTAAAAATGCAGTGTAAT TTTAGAAAATCTAAGAAAATTAAATTTTAATTCGTTGTTAAAAGTTAACAACGCGAATGAACCAAAGTTGGT CATGTGATCAGTTAATCATGGATTTAATTGGGGATTAAATTAAAGACGCTTCTGTGTACACCGGTCCTAAAG GTTTGAAATGAAATTTAAATAGGTAGATAAGGTGATATTTTTTTGTCATTTATTTATTTGCATGCCTACCACCT TTGTACTAGTATGTAGCTAAAGACATTGTGCGTTTCGATTGGGACATTTATGATCATGTGATACATGGAATAA ATTACGTACCTATACCTTCCATTGAGAAAAATTGTACTTTTTTTTCCACTTGAATTATAATAAAGACTTAAAA GTTTCAATAACATGGTTGTTTTTCTAAACCTTGAGCTTTCATCCACACCCCGTGTCTCCTTCGTAAACCCACC ATTACCCATTTCTTCCCGAAAAGCGATCCATCCTCGTCCATAAAAAGGCGTATCTCGTCCGCGATTCCGTCG ACATCATCTATAAGAGAACACCACTTTATCCATCACTTCTCGTTATCCGGCACAAAGCCTCCACAAGTCCAC ACTTTTGAGTGACACCGACTCCTCCCATCGCTCCTCCACTCCCCTCCAATTTCGCTCCCATTGGCCTGGTTG TAACCAAGTAACCAGAGTCCCATCCCCGTCCGCGCCAATGGTCGCCTCGGCAGTCTCGCCTCAGTGTAACC GCGGCCTCCACCTGGCAAGGTGCGTGTGATGGTGCACTAGCCCAAGCTGGCAACCCATggtaccggtcgccaccatg gagagcgacgagagcggectgcccgccatggagatcgagtgccgcatcaccggcaccetgaacggcgtggagttcgagctggtgggeggcggagagggeaccccega gcagggecgcatgaccaacaagatgaagagcaccaaaggcgecetgaccttcagcccctacctgctgagccacgtgatgggctacggettctaccacttcggcacctaccc cagcggctacgagaacccettcctgcacgccatcaacaacggeggctacaccaacaccegcatcgagaagtacgaggacggeggcgtgetgcacgtgagcttcagctacc gctacgaggecggecgegtgatcggegacttcaaggtgatgggcaccggettcccegaggacagcgtgatcttcaccgacaagatcatccgcagcaacgecaccgtggag cacctgcaccccatgggcgataacgatctggatggcagcttcaccegcacettcagcctgcgegacggeggctactacagctccgtggtggacagccacatgcacttcaag agcgecatccaccccagcatcetgcagaacgggggecceatgttcgecttcegecgegtggaggaggatcacagcaacaccgagctgggeatcgtggagtaccagcacg ccttcaagaccccggatgcagatgccggtgaagaataaagcggecgcgactctagatcataatcagccataccacatttgtagaggttttacttgctttaaaaaacctcccacac 
ttcccctgaacctgaaacataaaatgaatgcaattgttgttgttaacttgtttattgcagcttataatggttacaaataaagcaatagcatcacaaatttcacaaataaagcattttttc actgcattctagttgtggtttgtccaaactcatcatgtatcttaagcttggegcgec

\subsubsection{Vector map of the 'Tc-six3 5down' construct}

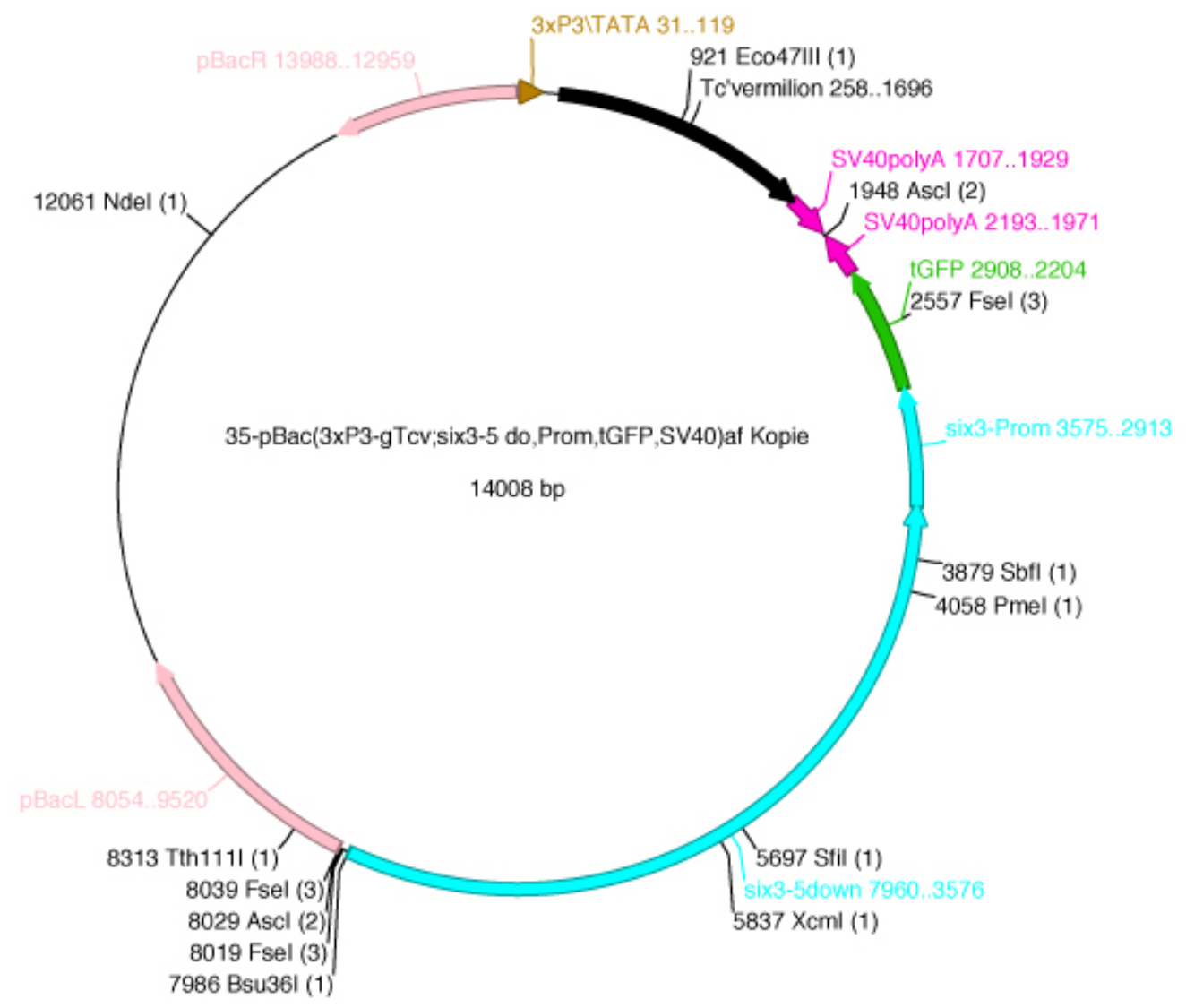

$>$ Tc-six3 5down::tGFP

ggcgcgccaagcttaagatacatgatgagtttggacaaaccacaactagaatgcagtgaaaaaatgctttatttgtgaaatttgtgatgctattgetttatttgtaaccattataagc tgcaataaacaagttaacaacaacaattgcattcattttatgtttcaggttcagggggaagtgtgggaggttttttaaagcaagtaaaacctctacaaatgtggtatggctgattatg atctagagtcgcggecgctttattcttcaccggcatctgcatccggggtcttgaaggcgtgctggtactccacgatgcccagctcggtgttgctgtgatcctcctccacgcggcg gaaggcgaacatggggccccgttctgcaggatgctggggtggatggcgetcttgaagtgcatgtggctgtccaccacggagctgtagtagccgccgtcgcgcaggctgaa ggtgcgggtgaagctgccatccagatcgttatcgcccatggggtgcaggtgctccacggtggcgttgctgcggatgatcttgtcggtgaagatcacgetgtcctcggggaag ccggtgcccatcaccttgaagtcgccgatcacgcggccggectcgtagcggtagctgaagctcacgtgcagcacgccgecgtcctcgtacttctcgatgcgggtgttggtgta gccgccgttgttgatggcgtgcaggaaggggttctcgtagccgctggggtaggtgccgaagtggtagaagccgtagcccatcacgtggctcagcaggtaggggctgaagg tcagggcgectttggtgctcttcatcttgttggtcatgcggccetgctcgggggtgccetctccgecgcccaccagctcgaactccacgccgttcagggtgccggtgatgcggc actcgatctccatggegggcaggecgetctcgtcgetctccatggtggegaccggtaccATGGGTTGCCAGCTTGGGCTAGTGCACCATCACA CGCACCTTGCCAGGTGGAGGCCGCGGTTACACTGAGGCGAGACTGCCGAGGCGACCATTGGCGCGGACG GGGATGGGACTCTGGTTACTTGGTTACAACCAGGCCAATGGGAGCGAAATTGGAGGGGAGTGGAGGAGCG ATGGGAGGAGTCGGTGTCACTCAAAAGTGTGGACTTGTGGAGGCTTTGTGCCGGATAACGAGAAGTGATG GATAAAGTGGTGTTCTCTTATAGATGATGTCGACGGAATCGCGGACGAGATACGCCTTTTTATGGACGAGGA TGGATCGCTTTTCGGGAAGAAATGGGTAATGGTGGGTTTACGAAGGAGACACGGGGTGTGGATGAAAGCT CAAGGTTTAGAAAAACAACCATGTTATTGAAACTTTTAAGTCTTTATTATAATTCAAGTGGAAAAAAAAGTA CAATTTTTCTCAATGGAAGGTATAGGTACGTAATTTATTCCATGTATCACATGATCATAAATGTCCCAATCGAA ACGCACAATGTCTTTAGCTACATACTAGTACAAAGGTGGTAGGCATGCAAATAAATAAATGACAAAAAAATA TCACCTTATCTACCTATTTAAATTTCATTTCAAACCTTTAGGACCGGTGTACACagatctATACTCCTCGCCACTG ACTTGCTCTCTGAGCTTTCTGATCAGGATCTGCATTTACCCTGTCTGCTTTCAAACGAGCAGTCCCCAAACA AAGCCACATTTTTGCCAAAACACTTCGAAAAATTCACTTCAATTGGTTGGGGTTATCGATTGTGTGTGGGGC CGACTGTTGGGAACCTCTGGCCGCCCTAAGCCGGTATTCAGTACGGTGTGCTTTTAGCAAAACACTTCCGA 
GCCCGACGTTAAGCTTCCACCTTTACACCAGCTTTATAAAGTCGTTATTCACGTAATAATATTGTGCCTGCAG GAAGCTAGGCCCAAATTATCGGGTGTAAAATTTCTCATTACGTAATTAAAGACACGGGAAAAGAATCCTTGT TATTTCCGTGCCGAGATCAAGTCTGACAGCGAATTTGTCGAATCCTTCCCGAAATCGGATTAGAAACACTAT CTAGCGGCCAAACTAAGATCGGATCGAGGTTTAAACGAGACGATACGAAGTCTCGCTTCCACTGTGTCGAA ATAAGACGCCCTAACAAGACGGAAGATCCGCGCCGGATTTTTCTAAGCCACAAATAGTTATTTTTGCGCCGA AACATTAGATTACATCGAGCTGATCGATCGATAACGGTTAACACGAAAGCACGCCTTACTTAAGAACTCATT TACAACCGCCTTAATGGCAATTTAGACACCGGATATGATGCCAGGAACTACCACCAGATTTTATCTCCATGTC CGGTCACTTCCACCTCACACCCAGCGCGAGGAATGCAACAAGAAAATAATGCCTGGAGAGCAAATAACTT GTAAATATTGTAGAGGGTGGCGCAAAACGCTCGAAATTAAGTGGCGTAAAGAGCCGCCTGCATGAGTGTTG GCAAACATTTCGGGTGTTAAAACACTCGATGCCCTTTTTTAATTAATAGGGAGGGGAAAATAGTAAAAATAG TCCCGTGTCATTAATCATGTCTGACATAAATGTATTAGTATTTGTAATTTATTTGTTTCATGCATATTGCATACA ATAATCTATTGTTTAGATTTTATCTCCATTGGATACAACAAATATTTTAGTTTTTATCGTAGGTGCTGTTGTGTG TGTTTTTACAAACGCAATTTGCGTTTCAGATTAATTACAAGCTTTTGTAATTACTCTAAGTAATCTGATATAAT TAATTCGTTACATATTTATGAGAATTCAACAAAAGCTGCAATATTTCACGTTTTTTCGCAAATGGCGCATTCG AATCTCATATCCACCCGAGATGAGTACTCATGTCCTGCAGCAATGTGTTAGCGAGGCAAATTGTGTTACCCA AATGTGATTCTTACCCCACAGAGAAGTACATTGTACCGTCGCTTTGTATAACCCTATAATATACTATTACTCTG ATTAAGTTAGGGCTGCTTTCCCATAGTTCACAGAACATGAACACATCAAATCTCCCTCGTCATCTCTCCAGC CTCTGATTTGTTTTTTCGATAAATCCGGCCAAAATCTCCAAGTACGCACTTGAATAAATTAATAACCGAACCG TTAATCGGCTTGTTTATTTATTTAAAGTGTACACAAAGGAATTTTAATTTGGTGGCGTAACGACGTGAAACTT GCCATTTTCACTATTCAGACATTAATCACAATTGCTGATAGCACAACTTTCATTCATAACTAGAACAGGAAAC GCCTGCAACACTCCGATACTTATCGCCGATTTTCCAAATTTTGCGAAACCAGCTAAGCTGCTCTAATCCTAAT CCTTTGTGATCGTCCAAACGATGGGAATAGCCCAAAGTTAAGTCGGATTGGGTTTTCCCAACGAGTTATTAT TTCGACGAAGAAAAAATTTCACGGTGGCATCCGACGAATTAAAGTCTGTTTCACGCTAATAAAATAGAAAC GACGTATAGTAAGCGGCGGATCGCTGCCTATGCTGTAATAATTACACGTTACCGCTTGATTTACGGCCTATTG AACAAGACTACCGGAATCTTTTATTGCCGGTTGGAGCAGCTCTGTTTACATGCTTATCAAAGTATTATATCAC TGTTTATGATGACCGTTTTAGCCGAAGAGGCCAGATTATGTTATTAGGCGAGGAAACACTGCGAGGAAATG GCCAAGTAGGCCTGGTCACGATATGTGACCCATGAAAGTATTTTGACCATTTTGGCTTCTCTACTAACAAAT GATTAAATTACACATTTTTTAAAAAAATTTACATAAGTACCTTCTAAGTACTTTTACTCGTAGTTAGCCAACA CATTCGTGGAAAGCACTGAAAATCAATTTATTCCCAAAAGTATTTTGAAAAAACAAATGTAAGCTCTAATTA ATGATTGTCTATTCATTTTTTTTCAGAGTTTTAAGCTCAGTTTTCTGCAAAAATCAAAAAAATTCCTGAAAAA AATTGAGTTGTGTTAGTACGTATTTTACGAAAAAGAAGAGATTGTCAGTTACAGTCACTGTCTGTTTTGATA GTCTTTCATTTTTGCAAGAATCAAAAAATTTATGATTCTTCTAAAAGTGATCCAAATCGACCAGAATTATTTA ATTCTGGCCCTTATATTTGTATTTAAAATGTATAACGATTTTTTGCTTAAAATCACGCTAAATTGCTTAGGAAA ACAATTTGAGTTATTCTGAAAATGATTTTAAAAATGCTTTTAATCAAAATTGCTTCGGCTTCTTCAAATAAAT AAAGAGTAACAAACTAGAAGTTTGTGGACACAGTGTTTTTTGTCCATTTCTCTAATTATATTTTTTGGGTTTA AGAACTTCTTAGATTTTTTTTTATTGTCAGTCGATTCAAATTCTTTTCGAAACATCTCAAGGATTACAACGCT CTTCATAAAATAATAGAATAATTTTATGCTTCAGATCTTGTATTTCTTCCCATTTTTTAAAACTAGAATGTTTG AATATCTAATAAAAACGGATTAACTATATTTGTGGATTTTTATTTTATTTTTTTTTGTTTCTTATTTTTATTTTTAT TTATGTTTAAGTGACGTTAATCTATTCTTGATTGTGATCAACTTTTCAAAATAAAAATTTTTCTTCATTTTTATT TCTATTTTCATTGTTCTCATTTGTCGCCAGGAGATTAGATATAGGTAGAAGAGCAGCAGACAAGTTGTCCTA GCTGTTGAATGAAACTTTCCTTACTTATTTATTATGTTTATTACTTTTCTGTTAATAAAGACATTACCACCAAA TTTTAATGGATACAATGTACCTAATAAATCGTTTTATTTCGCTATTTTTTTCAGCAACCCTATGATATTTTTATA ATTCAAAAATGGTTCTTACAAGACATTTTATGAAACATGTTTGTAAATATCTATGTTTTCTCTGAAAAAAAAA ACAAAGAAATCAAGCTTACTTATTATTATCTTCCATTTTGTTGGTTTATTAAACTAACTTAAACTTAAATTTAC TTACTAGCTAAGTGATCGCAATATAATTTAATCAACAATAAAAATTAATTATTTTTTAAACGGTTTTCTAGAAA ATAATTCCCAGTGTTGGCACATATACAAATTGTGTTTTTTTTGATAAATTTTATTTTTTTTAAATTCATAAAACT ACTAAAGTCTGATAGTAAAATTAAAATTAATTATTTATCATTGCGTTAGGAAACGATTACTTAGTGTAAAACA TAAAAACATTAAAAAATAATGAAAACTGAAGAAGGAGCTCCAGCAGATTCCTTTTCATTACCTTAAACCTTA AGTTTATTATCCTATCGACATTACATGCTCGTTAAATTGACTTTCAATTGATTATTGAGTTTATTCTATTTGTTT TATGCAGGATAATTGATAATATAAACAAATTCTTAAAGTTCAATATTTTGGGAACTTTATATTGGAAGAAAAA AACAGTCGTATTGTCCAAATCTAATTGTATGTTTTTATTTTTATTTTTAAAATTTTGTATAGGTCTCAACATTAC AGAGATTACAAATAGCACATTTAACTATCTTTTTGCTGTTTAAAATTAATTAAGCGTTTGGCATTTAACAAAT TAAAATTTTAATAAAATTTTGATTTAGCAATTTCACAGGTAATCAACGTTTTACATGAATTTTTGCCATTTAAA TTGAATCAAAACTGTTTTGGCTAATTGCACTGTTATTTTGTACACACAATTTTATACTTTTCAATTTTTCGCTA TTATTTCTCTTGATTGTTTCACAGTAATGTAATTTTGTACTAATTTGAGCTTGTTTAATTGTTTGGTATCTCAAA 
TAGAAACCAGGCCCAGTAGCAGCTACCCGTGTTATCCACGGCCTTCACTTAAAGTTTCCTCCCGAAAGTAA ATATTTCACGTGCGGTAAGCACCTAGTGATTAATCTGCTCCATCTCGCATCggatccatatatagggeccgggttataattacctca ggtcgacgtcccatggecattcgaattcggecggectaggegcgec

\subsubsection{Vector map of the 'Tc-six3 10down’ construct}

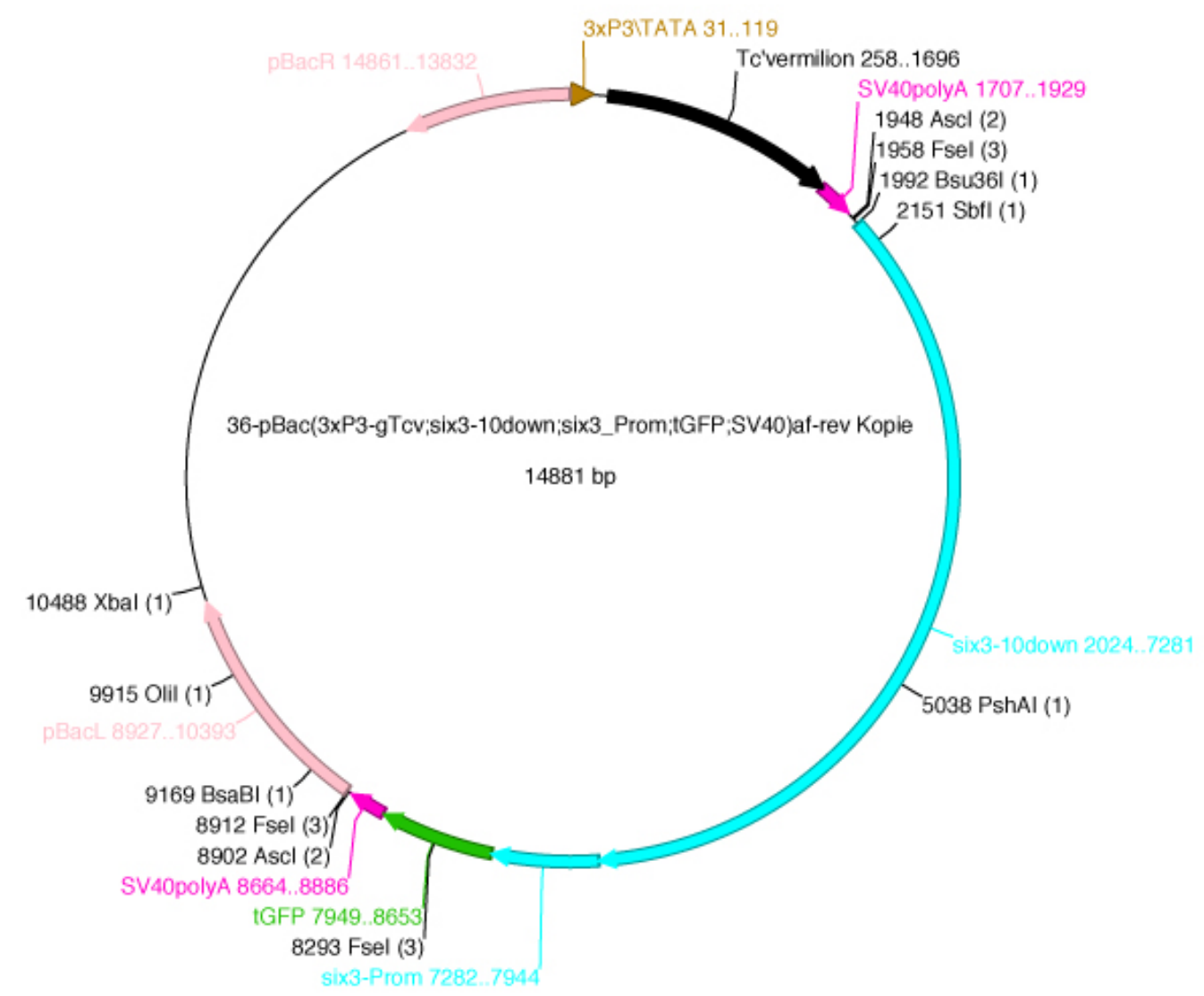

$>$ Tc-six3 10 down::tGFP

ggcgcgectaggccggccgaattcgaatggccatgggacgtcgacctgaggtaattataaccegggecctatatatggatccGGGAAGGATTCGACAAATT CGCTGTCAGACTTGATCTCGGCACGGAAATAACAAGGATTCTTTTCCCGTGTCTTTAATTACGTAATGAGAA ATTTTACACCCGATAATTTGGGCCTAGCTTCCTGCAGGCACAATATTATTACGTGAATAACGACTTTATAAAG CTGGTGTAAAGGTGGAAGCTTAACGTCGGGCTCGGAAGTGTTTTGCTAAAAGCACACCGTACTGAATACCG GCTTAGGGCGGCCAGAGGTTCCCAACAGTCGGCCCCACACACAATCGATAACCCCAACCAATTGAAGTGA ATTTTTCGAAGTGTTTTGGCAAAAATGTGGCTTTGTTTGGGGACTGCTCGTTTGAAAGCAGACAGGGTAAA TGCAGATCCTGATCAGAAAGCTCAGAGAGCAAGTCAGTGGCGAGGAGTATCAAGCAAAAGAGGCGAGAA TCCCCCGACTGCCTCTAGCCCAAGTGGAACTGTATCCCCTGCAGATTTCAATTCTCGCTTCTTTGATCCGCT CCAATCGACCTACTCCAGTATCACACTCCAATAACACTCCTCGCTTCCTAAGAAAGCAACATCTGGACTTGA TGTCAAAGGAAAATACGACGAGAATTTAAATAACTAGTGTTAATGCCGGCGGATTTTCTTCACGCGCTAACG CTGACAAATTTATTGATGATATTGAGCACACAATTTTTAGCGATTCGACAAACTGAGACTTAGCCAAATGTA CAGCTGCGAAAATTTACATAACAAGGACAAAAATTATTTTGAACTACTACACTTGACTAGGTTTTTTACGCG ATAATTTACTTACACAGAATATTAAATGCATGTTTGGCTTAATTTATTATGGAATTTTTTTTTTCTAACCTGTGC CAACAAAGATTTTCAGTAAACTTTCATTCAAATTCGGATGAACTTTATTTTTGAAAATAATAAAACAATTTTG ATGTCCATTTGTGATCGTCCAAAACGCTTATTTTGTGTTATTCTGCCTAAAAATACAATGTACAACCTATTTG ACAATTTTTTGACTAATTTTGAGTATTTAAGTAATTTGTTTTGGCTATAGGAAGATACTATGTGATTCTGTTGG TTACTGATTTTGGATTTTTTTTTGCATTTTTGTTCGCTTTTGACTTCTTCAAATAATTCAGACTAACATAGTTT AGTTATGTATTTTGAAAATGTTGGCTCAGGAAAATACATCTAATAATTGAGAACGATGAAAAAAATATACGA GCTTTATAATAAATACCTAGTACCTACAGGGTGAGTCAACTGTGGGTTATTTCAGAATTTCCCCTAATATGCA ATAATTCTGATTACAGCTGACTAGATCGGTAATCGTTAAAAAAAATCAAAATAATCCACGATTACATTGTTCT GAGTATTACCAAGTTTGTTTTAATAGATTTGCTAAAATTTTATTATGGACTATTATTATTTAAAATTATTTGTTG 
ATTCAATTAAGGTTTTTAAAAATTAAACAAAATCTCCAAGTGGTAAATGCAATTAGTATTTTTGATTGCATCT TAGGACAAGTTAGAAATAACCTAATAACTATTGACTTACCTTGTATACGTATTTTTTGAATATTTTTTTAATTTA TGAAAATCAATATTGGAATTAAATATGCAATTTAAGGAATTTTGAAGAATCCGGCTTCAGGTAATTTTAATGA TTAGTTCCTTTTTTGGTTATTTACAACATTTGTTATGAGGTCTTTGATGTTTTGGGGACATGTTTTTTTATTCAT AGGTATTGTTTCAAAAAATGCTTCTACTGTTAGTAAAAGATTGACGGAATTTTTATTTTATCAGAAGGAAAG ATATAAAACTATAGTAAATGCTTCTTCAAAGACTAATTTTTCAAATTAACTATACAATAGCCCAAAATAGTTT GACTGTAGAAAAAAATGTAGAAAACATATGAAGAAAATTAATATCTCGATGTAAACTCAAGTGAATTTTTTA GTCCATATCTGAGCGATATTTAGTACATACTTGGAAAAAGGCAAAACGAAGTTAAAAATTTGAATGAATCTA AGAAAATTTATTTTTGCTGCTGTAAGACATTTCACAATGCTCTTGCATGTTTTAATTTTTTAAAGAATTTTCCG AGATCTTGAGAACTATTCAGTCTAAATTGTCTTCCCCTGATCTAAAGTCATTTCAAAAAGTCTGTGGTTTGA GTATTACCCTCGTATATAAAAAATTAATGTTACATATTAAAAAATTACTCATGTTATTCATGTAATTTGAAATGA GGAGCGAACTAGCTTTACTTTTGCCTCTCTGATTAAGATTTAATTTGCATAAATTCGCAAAAAATAAATTATA CAATTAATAAATTTAATTAAGCTCCGTCAGGCAATATGTAATGTACTTAATTATACAAACTGGAAGTGCATAG CAAAACCATTTCAACCACCTGAATACTAATTACAACGTATAATTTTAAATATTGTTTTGTACAATGTGTGAGT TTGTAATTGGCTTCAGAAAGAGAAGCTTCTTATGGCGTCATGGCTATAATGTTCGGTCTTATGACAGGTCAAT TTCTCTTAAGCATAATATGACGTATCAATATTTAAATTCGGGGTCCACATTTGGCGCTGGCTCCGTTATCAGCC TTTTAATCTCCTCAAACCGTTTCAAAGCATTTATTAATTCACGAGAGCCATCCGCGTGCCTAATAACCATCGA ATGATCTAACATTTTCTTATCTCCATCATTCGCCTCGTTTTTTAATTTCAGAATCGATTATCCGAGCATGAGTG TAATTTTATTACCCCGGGTATGCATCACATGTTTCAACGCCTTTCAAACCACCTAACATATTCAATTAAGGTC ATCGGTGATTGTCTCAAAATCTGTAGCATCTTCATTTAGATTAGGCCGGAATGACTTGCATCCGCCGCGAAAT AATTAAGAAAATATCAGCGAGATAACATCACAATACGACAAATCCAACTGACGCGCATGTAACGGTAACGA GCCGTGACAAAAGTCCGGACTAGATGTGTCCGGAGCTTTTGTTTCTGGCACCTCCGGAGGGCTTTGTGTGT GTCGTTTATTTGTGTTTGGCGGTTTGTTTGGTTAGCGCTTTAGTGGTCAAGTCGATTGCTGATGGGTTCTTTT GTCCGCGACAGGACGGGTTTCTTCACCAAAACCGATCGAAATAAATAAACAGCGCTGAGAGGCTTATTAGC GAAAATTGCTGATTTGAATTGAAACAGCAAGTGAACAGTCTTTGGAGGTGCTCCACTCTCTGTTTTGTTTAC ATAGGACACCCTCGTTTGTTTAGGAAATCGCCAACCTTGTTGTTCATAAACACGCAGTATGAAAGCACGCG TAATGCTACCATTCTTGGGCTAATAACATTTATTTTTTCAATAAATTTGCCCCTTATAAATGTAACCGAAGTCA AGACTCCCTCGTGACACATGAAAGGTGGACTGTCCGACGCAGTCCGCGCTATTCTGATTGAATCTGAAAGT GATTGCTTTCGAGCCATTCCCAATCATTATGCGCCAGAAATAAAAATTTTACGACACTCTTTAGGGAAAAAT CGCAGTTACGTTACCGTGAACGCTCGTAAACTTTAAAATAACAGCTTGTTGAAGTTACCATAACCGATTTAA ACGTCGTTTAAAATTATTGATAGCGACGATGGAGTTTAACGAGGACACGTCATTGTGTTCAAATCTTTGTTC AATATTTACTGACAGGAAGAATTGTTTCTTGGATAAGACGAGAAAGCAATCTTCTCTAGTAGCAAAACAAA CACAAAACGTGTCGTTTATACACAATAAGGAGGCGACTCGACAACGCCTCGGCTTTGATGACGATGGGAAC GAAAAAATTAATTAATATCGATCAGCGGATGGATTAATAAGCAACGATTTTTGTTTACTAATTAATCATTTTCT TTACTGGTTTATGGTAATAAACTGGCAAGAAGTATGAACGGTTAGGTTTAACGGTAAAGCTCGATGTGTTTT CCGTGTGTGGAGACTGGGGCAACATGTAAATATCACAATTATTGTTTTAATTAGTTTAGTTTTAATATATTATT GACTCCACTCCTTTAAGTACATCTGCTATTTTTTAACCTTCAGGAAGGTGAAATTAAAGTGCCATCAAAACA TTGCCAGGTTTATTACATTCAGAATCACAGTAATTAAGAGAAAAACGTCTTAGGTGTAATGAGTCTCATATGT TTGTGCATAACAGTTGCACAAAAATCATCGAAATTTTATGATTTACAATAGATATTATTTAACAAATTTGTCCC AATTATAAAAAATATTGTTAAAAAGAGAAATGGTTCAAATGATGAAATTTCAGTGAAACAATTCACATTTGA TTATATTTTTATTGTTTTAAAGTTACTACATATATTTCATTGTGATTTTCAAGCAAACGTTATTGTAAAAAACAT CAGCTATTTAAAATAATATTGTGTTTACAACAAAAAATTATATCCAACAATTCTGCTATCTCTAAAGCAGTCTT TTTTTTATTTTCCACTCCACTCACTACTTTATTGGCATAGCAAACATATATTGTTATAAGTATTGCGTGTAAAA ATTGTTGGGATAAAATGGTGTTGGCAAAGAAATATTTTCCTTTACATATGTGTGGAAAAAAATTACTTTGCCT GCTTGTAAATATAGCAAATGATTATTATTTATGGAACGTAATTTCTTGATGAAATACCTAAATTTTTTCGCCAG TCTATCAATAAATATTGTTTTCCACCTTCCAATTATTTTATGAAAGTACGTATTTTCTCTCCCATTTTTCCTCCT CGGGAGGAAATAACTAGGGGATCGGATTTTTAAAAAGTTAAAAACTTTATTTAGGTGTCGGAAAAGGTAAA AAAACAGACATTACATATTAAAATCTGGCCATTTAGGATATATTTTTTTATTAATTCCTACTGCAGAAAAAATA AAAACATAATTTTTAATGCAATAACTTCCATTACGAAAAAAAATAAAACAAAATACGTATTGTAATTACTTAA AATTTAGTCACAAAGAAAAAGCTTTAAAGAAATTAATTAATTGTTGAACTTAAATATTGTTCTAAATTTGCTA ATGTAAAATTATTTTATTTTACCGTTAAAATTTTTCAGCTCTATTTAACAAAAATTTCAGAGGAAAAAAGTAA GTTTTTTTTTCAAAGTGGCTTCATTAAAATAATACAGAGCAAGTCATCGTGAATCTTCAGAAagatctGTGTACA CCGGTCCTAAAGGTTTGAAATGAAATTTAAATAGGTAGATAAGGTGATATTTTTTTGTCATTTATTTATTTGCA TGCCTACCACCTTTGTACTAGTATGTAGCTAAAGACATTGTGCGTTTCGATTGGGACATTTATGATCATGTGA TACATGGAATAAATTACGTACCTATACCTTCCATTGAGAAAAATTGTACTTTTTTTTCCACTTGAATTATAATA 


\begin{abstract}
AAGACTTAAAAGTTTCAATAACATGGTTGTTTTTCTAAACCTTGAGCTTTCATCCACACCCCGTGTCTCCTT CGTAAACCCACCATTACCCATTTCTTCCCGAAAAGCGATCCATCCTCGTCCATAAAAAGGCGTATCTCGTCC GCGATTCCGTCGACATCATCTATAAGAGAACACCACTTTATCCATCACTTCTCGTTATCCGGCACAAAGCCT CCACAAGTCCACACTTTTGAGTGACACCGACTCCTCCCATCGCTCCTCCACTCCCCTCCAATTTCGCTCCCA TTGGCCTGGTTGTAACCAAGTAACCAGAGTCCCATCCCCGTCCGCGCCAATGGTCGCCTCGGCAGTCTCGC CTCAGTGTAACCGCGGCCTCCACCTGGCAAGGTGCGTGTGATGGTGCACTAGCCCAAGCTGGCAACCCATg gtaccggtcgccaccatggagagcgacgagagcggcetgccegccatggagatcgagtgccgcatcaccggcaccetgaacggegtggagttcgagctggtgggcggc ggagagggcacccccgagcagggccgcatgaccaacaagatgaagagcaccaaaggegccctgaccttcagcccetacctgctgagccacgtgatgggctacggcttct accacttcggcacctaccccagcggctacgagaacccttcctgcacgccatcaacaacggcggetacaccaacaccegcatcgagaagtacgaggacggeggegtgct gcacgtgagcttcagctaccgctacgaggccggecgcgtgatcggcgacttcaaggtgatgggcaccggcttcccegaggacagcgtgatcttcaccgacaagatcatccg cagcaacgecaccgtggagcacctgcaccccatgggegataacgatctggatggcagcttcaccegcaccttcagcetgcgegacggeggctactacagctccgtggtgg acagccacatgcacttcaagagcgecatccaccccagcatcctgcagaacgggggecccatgttcgccttccgccgegtggaggaggatcacagcaacaccgagctggg catcgtggagtaccagcacgecttcaagaccceggatgcagatgecggtgaagaataaagcggecgegactctagatcataatcagccataccacatttgtagaggttttactt gctttaaaaaacctcccacacttcccctgaacctgaaacataaaatgaatgcaattgttgttgttaacttgtttattgcagcttataatggttacaaataaagcaatagcatcacaaat ttcacaaataaagcattttttcactgcattctagttgtggtttgtccaaactcatcatgtatcttaagcttggcgegcc
\end{abstract}

\title{
7.3.7. Vector map of the 'Tc-rx 10up’ construct
}

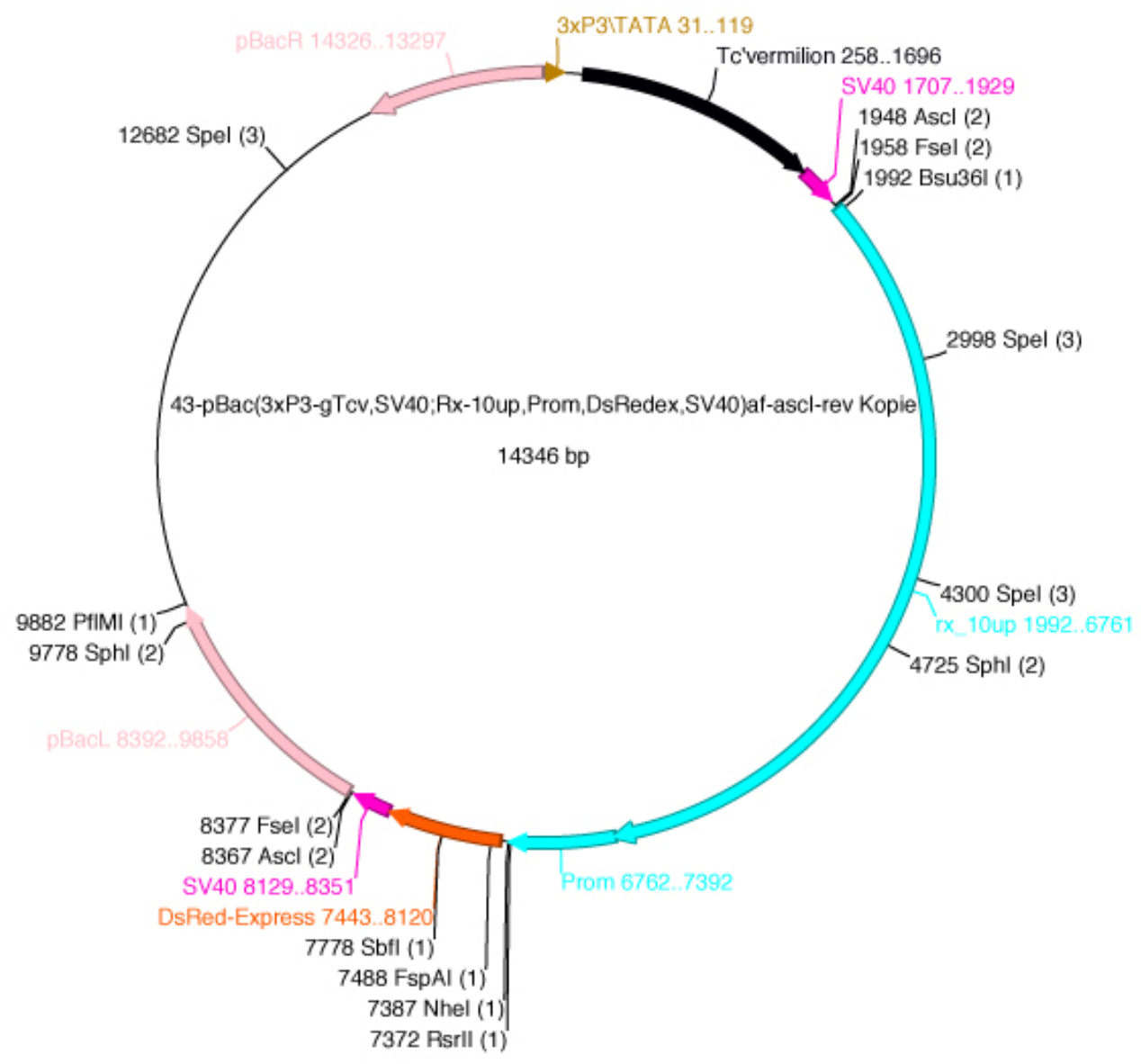

$>$ Tc-rx 10up::DsRedEx

ggcgegcetaggecggecgaattcgaatggccatgggacgtcgacetgaggACAGCAGCGACTCGTCACTCTGATATCAAACTGTTAAC TTAAACACTTACATTATTTATATTTACAATAATAATAATAAATAAAAAATAAAAATTAAATCACCGTTTTTATAC TAATTACCTATACCTAACTCCACATTAATTCCCGTAACTCTAAAGTAATTCAACCTAAGTAATTTTAAAAACTT CCATTAACAACAAAAAATCTATAAAAGCTCTCAGGAAATACAAAAATTGTGTTGAAAATTAAAGCTTTTCA AAGTACAAGAAATATTTATATCGTCTCCATTATCGTTGACATTATTGGTCGCACTAAAGCGATAATCTTCAAAT AAATTTAGTCGTTTTGAAAAAAGGACTCAAAAAAGTGAAAGGAACTATTCCAAGAAAGTTTTGCTATGTTT TTCAACAAGAAATCGTTAATATACCGGTTAATAACTGTTCATTTTTAAACCAGAACACAATACAATATACAAT 
AAAAATATTAGATTTTAACATTTAACATTCGGCATAATTATCTAAACAAAAGGACAATCATATTTTGTAGAAT GTTCGAGTTTGAAACCGATTACATGTTCGAAAAACATTATTTTTGCCAAAAGACTGCTTAAATATAAAAAAA ATTTCTAAATAAAATGGTTCACCAAAAAAATATTTAAGCAAAGCAAGTACGAGAATTGTTGTTTCAACCAAA TAGAGCGAGCTATTTACAACCTATTTGCGCTTTCCAAATGGAAAATCAAGACTTCCAGAAAAATTCATTTAC AATGCAGTGCATCCATAAAAATCTATTAATTCTTAATTAATTAATACTTCCAGATTATTCCAAATAATTTCGATT AAAATCCAAATATCTACGAGTATATATAGTACGTACCTATTTACAAAACTAATTTAACTTTAACGGCTTCCTAA TAAAAGTAGGTACTCGGATGTTGAATATTCAATCATCCACAAAAATGACGAATTCGAAAAGGCATCAAATCA AAAATAGAATTTTACTAGTGTTATGACTAATTTCAAAATATAAAACAGAAAAATTTAAGTTTTTTGTCGTTTT ATGCTTTAAAGCAATGCAAACTATCGCTAAAAAAAACTAGACACGGTAAATTTTAAGGTTGTACGTATATTT TAAAAGGGGACAGTAGAGTTCAAAAAAATTTAAAACAACTTAACACTCAACTTGTTTTTTCCGAATGTTTA GAGGAATTTAGTCAAAGAAACTTAAATTTCTACACAACTTTAAACTTCACCACAAAAAAATGTATAGGAAT GTGATCTCTGAGGTTAGTTTTTTAAAACCTATTACATTGTTTTAAAAACAATAAGCTAAAATTTGCAATAATC ATCATAGAAAAAAAATGTCTTCATATTAAAAAAATTATAAAAAAATTTGCATGTTTTTTTTGTCGAGAATACC ATCAAATTGAATTTCCTCGCATACCAACGACTAATAGCAATGTAAACTTGAGCATAAAGTACTCAAATTACA CAAAATTCGTTTAGATTGCATTTTTTGACGAATTTTTGGACGTTAAATCGGAAGTTTCACTCTCTATATTTTAG AAACTATAAACATTCTGATAAAGCAAGTTGAGTTTTTTTGACTTATTTTGAGCTCCACTATTACCTCTTACAA TAATTGTACAATCTTTAAAAATTCATTTTGTAGTGTAGGTTTTTAGATCACATTTTGACACCTAATACGGTCGT GAACTGAAAAGTATTGTTGGTAGGAAAGTGTATTTCGTTATTTCCAGCAATTTTTTTTTCTGTAAACGATATC ATTTCGTGATCTTTATGGTTTTTAAAACTTAGATTGTAACTTTAAAAACTTTTTAGTTTTTATTGAGGAATTTT AAGGAAATAGAAATATGACGTTAAAAATTTCTTCTTTGATTGTTATTGAGGGAATTTAGTGGTACTTATTGGC TTAGTTATTAAATGATCAAAAGCACATGGTTAGCTTCGATGAAATTATGTGCGTTAACGTTTCCTACGGTTGT TTTTTGCCTGTTCTTCACACTTTATTACCTCCCGACGGTTTCAAATTTTAACATGTAAAAAAATCAGCAGCGC CACTGGCTTCTAAACTCGTTGAAAGTACATCATTAGTCGGATTAAGGAAGCTTTTGCGGTGTGGTGAGACT GTCACATACACAACGTCCCAAAAAGCGGTATTGTTGGCAAAGAATGGCTCAAAGCGGTCAGATACAAATGT AAACAATGGAAGCGAGGCGCCGATTAACCCCAGGGGGGCAATTCAGAGCTCTCCCTTCTTCATCTTGCCGT GCGCTTATAAACTAGTTATACCCTTATGTCATTATCCGTTTGTATTCGCACACATTGGGGGAAGTTGTCTATTT GTTGAACGATGTACGATTATATTGTTTATCGACAGGAAAAAGTAAAAGAAAAATTGGAAGAATCGAATTGTA GCTTCCTGAGTTTAAAAAAACGAGTGTCGTTTCCTGGATTTGAGCAGATAAGTGGAGAATGGCCCACAAAG TCTGATTTCCCCTCCCGTTGAGCCAATTGTATAGGACACAATACAATAACAACCAATAAAGTAATGCAAGGA GTTTGGAGATTGCAGATTAGCAAGCCTGATAGGATTTAACCAAAGGTCGGCACAAAGCACATGTTCTGGAC AGATACGGTCTAATATAAAGCGAACACGGAATTCCTGTGATTGAATTATGGCCACGATAATGATTGGAGGCA ACCGCATGCTCAAACAAAGCAATCACCAATCTATAAAAACCAATTAATTTTTGAACGCCCTTGATGCACTTA TTGATCGCCGGTTCCGCGAGGCACAAACCCAAAAATTCAATCAACCAACGGATTTTTAAATCCACAAAACG ACCATTCGTGTTAATATCGTTAAGTGCCGTTTGTAGCATGTAATCATCCCAGCTATCACAAAGATCAACAGTC ACAAAATCACTCCATTCAATAGCAATAATAACCGATAACGTGCTTTAAAGTGATGCGTGCCATTCTGCATCA CATTACCTAAATTGAATGACGACGACTAGAAATCTCCTCGAAATCTATAGCGATTCTACCACTCTTATCTGCC CTTAATCTTGCCAACCCTAATAGAGACTTAAAAGGGTTCCAGGAAAGGTGTGCTTAAATAAAGCTCGTCAA CGATTTGATGAGATCTGACAAGCCATTATAGAGACACTTTTTAAAATAATTTAGCATTGCGCTAATTTGAACG CTTTTTAATGACAATAAAGCGCCACAGGACGCATACTTTTGATGACATCTGGACTGGAAATTTAGAATGTTA AAGGCACCGCTTTGGCATACCTTAAAAAAGGGAAATAGACGCAGGGATGACGGTTGTACACTATCATTAGG GATGATTGTACACGCCTGCTAGGTCCATTCAAGTTGTTGCAAATTGAATAAATTGCAGGGTTTACATATGGC GGTTTTATTTTTTAAGCCTCGTCAACGTTTACAAAGGTTTCGTTTCCACAATGATTTTGTTAATTATTTATAAA GTGACTGAAATGTTTTAAATTTTTTTTGCCGAATTAAATTAATTGTAAGGTTTTAGCTAGTTTATTTAATTACA AACTAACAAACAAAGTGGAACTTCTTCTTATTTGTGAGCACACGTTTGCAAATCTTTTTATAGGAGTGCGCG TACAAGAGGCATTGTGTTGTGTGTGTTATGATTTTATCTTTTAAACGTAATTAGAGCATACATTTGTGCCCTG AATCGCAATAATGAAGATCATATCTGGCGCATCTGCTGGTGTGGAGCATTATCGATAAAGTCCTCTTATATTTT GTCCGTATGAAGAAAAGAAGGATAGCAATCAGACACGGGCCACGTTCTCAGCGGGATACATCCTAAACCCC CAAACAAACCACAATTACAAAAGCCGATCTTTGCAGAACGTCTTCGAGTAAGGATAAGCCGCCTTTTGATA CTTGTTACAGTCCGAGATCCGCAAAGATACTGTAAATATAGAGGAATGCAAGACTCTTGAAATGCAATATTC CGACGACACGCTATAGGTATAGTGGCGGGGTTATGTTTGCAATCCTGTGGCTCTTTTCAAGTCGACCACAAT GGAAAACAAGCGTATTATCTGCTTATACTCCAGCTTACGTTTTGTTGATTTTTCGGTAGCCTCATTACTAGATT TTTTTCATAAGGGATCAAGCGTAAATGGGACGTCCCATACAATATATGTTTACCAAATACCAAGTGTTTGCAG GATAATAGAATAATAGTCTAAAACGCTTAATGCATTTTCAACTGTAACACCTTTTCGAGTGCTAGGCCGAGC CCACGTTTTATTGTGTGGCCGCGCTTTGTCCGCATAGATTGATACATATCTTCTTTTTCTCGAGCAGACTATGC CAAAATTTTATTTAAAAAAAATCTGACAGATATTTGCAATTGTAATTGGTTTCGGTTGTGATAAAATAAACAA 
TACAAAAGTAAACATTTTTATTTTCAACTTAAAAAAATACAACGTTGGGTACTTGCAATTTGTAATTACGAA CTAACATAGTACAAAGAATGAGGCTGCTGAAAGTATACCAACTTGTTCTGTATCTCAAAAACTAATAATGAT ACAGAAATTAGTGTTTGCTAATAAATATTTTATTTTTCTCATGTAATTGGGTTCATTATACACGAATTGAATAG GTACTATAGTTTATCTACTACAGCATTATTTTTTGACTAAAACGCTTTGTCGACGTATGTTGCCGTTGTTATCA AATggatccTCCTCTAATTATAGCTGAATACAGAGCCGAGTTCTTATACGTAATGGAAAACAAAACTATTACTAA TAATTTGCGTAATATGCTGTGTCAAGTTACATTATTTTAAAAAGTGAACGTTCCCAAAAAATAAGATTTCTTG AGCCAATTTATCGCAATTTAGGTCCAATTTGTCTGACCTACGAATCGATATTTCACCAATTACTCGTATTTCCA GTCCCCAAACCCGCTGTAAAATTAGTTTGAATATCTGAAGTGTGAACGAGGCAACACTTGAGACCCGTTGG TTTCCCAGTAATGGAATAACTGGCAAGCCCAATAACATATCTTATTGGCAGCCCCACTTAAAAACGAGTAGC AATAGCAACATGTACTACTTAATCCAGATTGTTGACACCAATTTTGAAGCTTTTATACTGTGAAATTTCCAGC ACAGACTCTATTACTGTTGGCGGGCATCCAACCTTTCGTCTCTCTCTACCCTCCAGGACTCCATGATGGATG TCCAAAGTGTTTCATTGGTCCTCGACGTATTGAACACGCCCCTGAAAACCGCCCCTAGGTATCCAAGCGTG GAAGCCGACTTCAGTCAGTTGATGTGGCGCGATGGAATCGGACCGTTGTGAAGgctagctatagttctagaggtaccgegg gccegggatcgatccaccggtcgccaccatggectcctccgaggacgtcatcaaggagttcatgcgcttcaaggtgcgcatggagggetccgtgaacggccacgagttcga gatcgagggcgagggegagggccgeccctacgagggcacccagaccgccaagctgaaggtgaccaagggcggccccttgccettcgectgggacatcetgtcccccca gttccagtacggctccaaggtgtacgtgaagcaccccgccgacatccccgactacaagaagctgtccttccccgagggcttcaagtgggagegcgtgatgaacttcgagga cggcggcgtggtgaccgtgacccaggactcctccetgcaggacggctccttcatctacaaggtgaagttcatcggcgtgaacttcccctccgacggccccgtaatgcagaag aagactatgggetgggaggcctccaccgagcgectgtacccccgcgacggegtgctgaagggcgagatccacaaggccetgaagctgaaggacggeggccactacctg gtggagttcaagtccatctacatggecaagaagcccgtgcagctgcceggctactactacgtggactccaagctggacatcacctcccacaacgaggactacaccatcgtgg agcagtacgagcgegecgagggecgccaccacetgttcetgtagcggecgegactctagatcataatcagccataccacatttgtagaggttttacttgctttaaaaacctccc acacttccccctgaacctgaaacataaaatgaatgcaattgttgttgttaacttgtttattgcagcttataatggttacaaataaagcaatagcatcacaaatttcacaaataaagcatt ttttcactgcattctagttgtggtttgtccaaactcatcatgtatcttaagcttggegcgec

\subsubsection{Vector map of the 'Tc-rx 5up' construct}

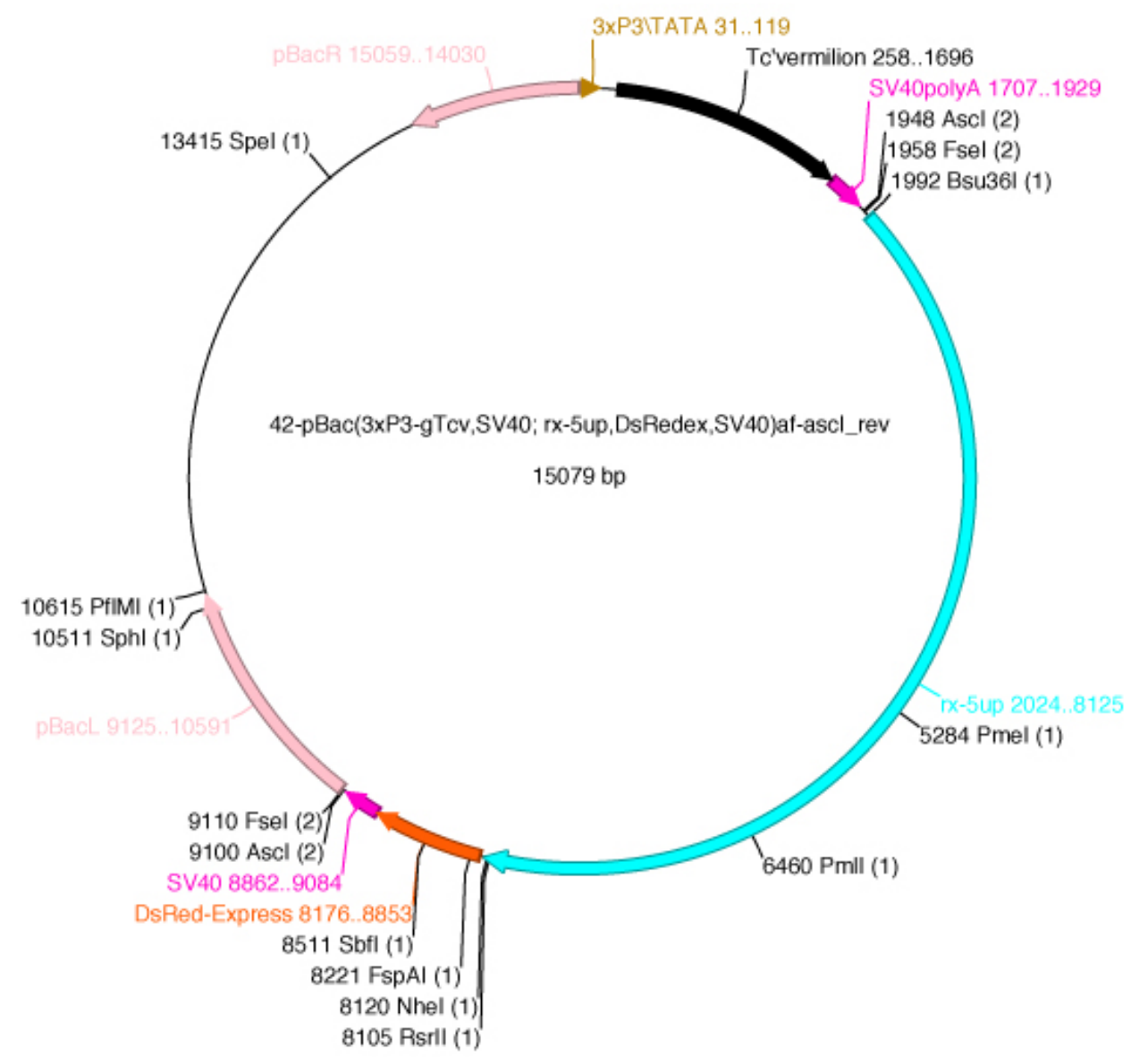




\section{$>$ Tc-rx 5up::DsRedEx}

ggcgcgcctaggccggccgaattcgaatggccatgggacgtcgacctgaggtaattataaccegggccctatatatggatccGGGATCAAGCGTAAATGGG ACGTCCCATACAATATATGTTTACCAAATACCAAGTGTTTGCAGGATAATAGAATAATAGTCTAAAACGCTTA ATGCATTTTCAACTGTAACACCTTTTCGAGTGCTAGGCCGAGCCCACGTTTTATTGTGTGGCCGCGCTTTGT CCGCATAGATTGATACATATCTTCTTTTTCTCGAGCAGACTATGCCAAAATTTTATTTAAAAAAAATCTGACA GATATTTGCAATTGTAATTGGTTTCGGTTGTGATAAAATAAACAATACAAAAGTAAACATTTTTATTTTCAAC TTAAAAAAATACAACGTTGGGTACTTGCAATTTGTAATTACGAACTAACATAGTACAAAGAATGAGGCTGCT GAAAGTATACCAACTTGTTCTGTATCTCAAAAACTAATAATGATACAGAAATTAGTGTTTGCTAATAAATATT TTATTTTTCTCATGTAATTGGGTTCATTATACACGAATTGAATAGGTACTATAGTTTATCTACTACAGCATTATT TTTTGACTAAAACGCTTTGTCGACGTATGTTGCCGTTGTTATCAAATTTGATTTTATCAAACATAATATAAAAC TCAAATAAATAAGTAATTTATGCCTCAAAGCTATTGATTTGATCGAGAATGGATACAGAAGAAGAAAATGAT ATGCAGTGCACACCCCCAGAAATTAGAAACACGCATACACTGTATACTCATTTAATAAAATACTACAGTGGA ACTTGGTTATAACGATCCCGGCTTGTAACGAATTCTCGGATATTATGGGCACTTTTGTTCCATATTGCAGCAA TATTTCGACAGGCAAATAGGCACACAAAATAATCAGAAATTAATAAATTTGAGCATAAAATTAAAAATTATT GAATTGTTAAAAAACTATTGAACATAGGTGTAATAATATGTAATCAAATACTTCTTCATAAATTATGTTAGTCC TTTATTTTTTATGTCTTTTTCAAGCTTTAGAATTTAACTTGAAAATTAATACATAAAGACGACGTTCGGTTATA ATCGAGGTTATAACAAACCAGTTCTAAATTTCGTCGAGTGTTCGTTATAACCGAGTTCAACTGTATTAAAAA AATTAGTTCTAGACTTGTTTTTTCTTATTTGAGTGTGTGTGTGTGTGTGTTATTTAAATACTTAATGCACAGTA GAGTGCCAATTCTGCGAACAAGAGGCAATTACAACTATCTATACCCAATATGAAACAGACGTTACAATAACT ATGTATCGAATATTTCTCGGTATCCATTGTATTTGTGTTTTCTATTTGGAACTGTTTGTCAAACAAAGCTTTGA AGCAGGTAGTGATAATGATGAAGCTTTACTACTTAAAAATGGCCGCCGTGGAATTATAGATACAAATAAATA GATAACATCTTTCGGTATTGAATAGCTAAGCAGCCTAATGCCTGTGTGATGTATTGGTGTGTAAAGTGTATAT TTTCCATAGCAATTTCCAATCAAATTCAATCAGTAAAAGCAGTTGAGTAAATAATAGAGTCTAACTGCGGTC GCAGTCGTGATTAGTTCTCCGTCTGTCCGCCGCAGGGTGGGGGCTCCACCTGCGCGCTAATTACCATAGTCA TGCGGCTGGGCTCCCGGAAATTAAATCGGTTTACGGCGCTCCAATATTTGTGTTACCAACATTTAAACAATT TAGTTTCATTATGGCGCGACTTTATTTGCGCCTTATTAGAGCTAAAGTAAATATGGCGGATGAATTTTTCGGG CGAATGAGTTTTAATGAAAATAACGCTGCGGAAAATCTGCGAGATTATGAGAGTTAATAGGGCCGAAGTCA TTTATTTGCGATTAAAAACACTATCACATCAGCAAATCGAATTAGGGGGTGCACAAAGAGTATAAAACAATC ACGGCGTCCGCACATACATCGCAACAGAGAGTAACAATGTGCCAATTTGGCCTTAAACGGCCCATTGTAGC TGGAAATATAATAAAAGCATTTTTATAGCAGCTCCCATCTGGCGTCCTATCTTGGAACTAATCACATTATGTAC TCCATTTTTGAAGCGCCCTGCCTCCAGCGTACAATTCAATTTAAAAGTCATTTGTACTATGCAGGAATATTGT TAGCATCGTTAATAATAATAATAATTTCATTTAGTACTCAGAGTATAAAAGAAAATTACAACGACGCCGTCGT GTTAAAAATGAGCGTCGTAAGCGCGTTGCGAGCAGGTATTTTATAATTTTTAACACCTTACATAAAAACGAA AAACGCTGCATCAAAGATGAAATTCTGATACGGCAGATATCGCTTAAGTGTTTATAAAGGCAGGGAAATTGA ATGGGGAAATAACATCATTACATTTTATTGCATTTGGCTCTTATAATTTATAATTCCAGACCCAAATGGTTTTT CTTTCGCAAACGCGGCGGTGCCGAAAATCCCAACTTTCACTCGACCCACACTTAATTGATGTTCAATTTTGA TAATGTTCGAGATAAATTGGTGTCACGGCTGAAGAGTTTAATAAAGGGTATATTGAAGGGTGATGTGTAAAT ATCGAGTGCGAATTGATGCATTTATCCTCGCAGCGCTGCAACCACTAATGACACTTCTCGGTCACTAATTTT GTCAACATACTGTTACTTTCATATGACATTATTCTGCCTCATCAGCAGATACTCCAATTTGCACAAACACGAC GAACCATGAGTTTAATTGCGAACCTAAGTACCTAATGCAACAAGCTGACCGAAAGCTTTATTGCAACTGCA AATAATTTCATTAGTCCTTTATGTAACCCAACAAAAACAAACCATTCAAATTAATAAAACTCCAAAAATGAA CTACGCGGAAACGGATCTGCACGTCAAAATATCCGCCATCGATAAATGATGCAAATGAAACGGCTTCCTCAT CCGCAAATGCAAATCATTTCTACATAAAACAAGTTGCAATTTAAATTCAGTAATAAACTAAGAAAAGAAATG ATATTTTTGTAAACACCTTAGTCGACGAGTCGATCGTGAACTCAGTCTTCGAATCATCTCAATCAATTGCTCC ATTGCGGCTAACGCTAACACAATTGAGACCTCGATTGAGACAATCACAATAGATTGGATTTGCAGTTTAGTA TTTTAGACCAACTTTATATTTTTATTTTTTTGAACTCAAAATACGAGTAATCAGTTAAAATTAACAATTATTTG TAAGATGAAAATAACATATAGCTTATTAAAAGAAGAACGAGTTTAAACAACCATTAGGTTTAGTGAAAAAA AGACTAATGCTTTATCAGATCACCATTAGGTATTATTACACATTTGGCTGCTCGAGAATTAAGCTATTAGCCAT TTTAATGAAAACTACTTCGAATATGTAAAATTTCATTTATATCGTCCAGACTTGCCACTTTGGCTTTTGATGTT AATATCTACGTAAATGTATACCTATTATTTATTAAAAGAGTAAAAGATTGTCAAATAGTTCGCATTAAAAAAC AAGTTTTATGAGACAGTCTTTAAGACACGAATTTTTATTTAACAAAACGAGTAAAGAATTATTTAAAAAAAT CAGTAGGTACCTTAAGATCTTATAAACTTTATAATTTGTTTCTTTAATGCAGTTTTATAAATAAAAATTTGAGA TTCTGTTCATTTAAAAACATGGTACTGAAAAAAAATTATATAGTCGTTTTTTACGTTATCTTCTTATTTAAATT TTGTCACTAAAAATCAAAATGAAGTAAAGCTTTTATCTAGTAACTCGCACATCTCGCAACTGTTTTGTCGCT 
AATCTGACAACGTTTTAACTGCGTTTTTTTGGGATAAAACAGTAAATCTGTGGCAGAAATTTACCTACTGAC AAATTTTGGAGCAAAAGAGTTTATTATTTCATAAAAAAAAACATAACAAAAATAATAAAATAGATGCTACAA AATGTATTGATACCTACCTACCTACTTTTCTGGAAATTCGAGAAATTGTTGAACATACTGTCTATTTTAGACA AACGTACCTACTTCTGTAATTAAATTAAAAGAAAAATATTAATTTATATCGAAAATTATGACGTTGCATTAGCT TACTGTATTTTTAAAGAATCAAATAAAAAAATACTAATTTTTTTTGCGCATGAAAAATTGGCCTGCCAAATTT TTGGATTACGATTTATTTTTCCGAGAATCTGTACTTAGAACTAAATCAAAATGATTTTACTCTGACAAAAATT TCTGATTTTTAAACACAGAGAAAAACGATCTCAGTTGAGGTCACGAACAATCAAATTCAAAGTTTTCCCAC TTGGACTCATGCCGTTTGATATTGATCTCATTTTTCTCTGTACTAAAGTCGTATTTGCTATTTTCCGCTTCAAC TATCTGTACTCTCAAAAACTACAAGATTGAAACTACTTTATTTTCTTAAACACGTGGACTGAAGTCGTTTTC CTCCTGTACCTTTCATACTCGTATGTCCTATTTTGCTTTTTCAACCTATATTTCCCAACAATTTTTTGAACTATT ATAATGTGATTTGGTAGGAAAAATTGAGAACAAGTAACTGTTGTGTCTATTCAAGATAAAAAAAAATCTGTT AGTAGGAGGTACCTACTGTTACGTTTTTAAATAATAATCATAAATTATCAGAAATTAGGTTTAAGTTCATGCT GTCCAAAAAGCCGGTAACAACATACTAAAGCACAAGCATTTTATCAGAACTGAAATTTAAAGTGCTAAATA CAATACAGTGTTTTACATAATTTTTCGAGGCCTGTTTCTAATTACGCACCCAAATACATAAATCACTAAGCAC TACATACAAAAAATACACATTTTATCTTTTAATTTCTGGAAGTAGTAGTTGTCAAACTAATCACTGTTTCATTA ATAGTTAAATAAATTGCAGTTTTTTCAAAAACAATTAGGTACCATGTCTTCTATTAAGAGACAGAATAAATAT GATTCTAATCTACATATCTATCTCAATCACTAACTAACATAAAACGTTATTTGAAGAAGTGAACAAATTAAAG AAAACTATTTCAAAATTTGTAATCGAGTTTGTATCGGCGCCGGCTTCGTAAAATTATGTAAAACACCCTGTA AAAAAGTTGGACTAAAAAAGCTGCTGTTTTTCTCCTGTATACACATTCCAACTACTTATGATTGTGAAAAAA CATAATACTCTATTATATTATATTATTATATCCGATTTAAAAAAGTTTGATAAAAAACAATTTTCGTATTTTTTTA AAATGTACTTATTTGATGTTGACTGTAAAAAATATTGAATTTTGTATCATATATGTATATATTTAATTTGGATGT AATTAACAACGAATGAAAGAGCAAATTAAATCGGTATTCCTATTAAAAATTAATAAATATTTGTCAATAAAAG AAATTGAAAATTATTTAAAATTTTAATGGGTAGTGATTATTGCTCTTTTAAATGTGATTATTCCTCTAATTATAG CTGAATACAGAGCCGAGTTCTTATACGTAATGGAAAACAAAACTATTACTAATAATTTGCGTAATATGCTGTG TCAAGTTACATTATTTTAAAAAGTGAACGTTCCCAAAAAATAAGATTTCTTGAGCCAATTTATCGCAATTTAG GTCCAATTTGTCTGACCTACGAATCGATATTTCACCAATTACTCGTATTTCCAGTCCCCAAACCCGCTGTAAA ATTAGTTTGAATATCTGAAGTGTGAACGAGGCAACACTTGAGACCCGTTGGTTTCCCAGTAATGGAATAACT GGCAAGCCCAATAACATATCTTATTGGCAGCCCCACTTAAAAACGAGTAGCAATAGCAACATGTACTACTTA ATCCAGATTGTTGACACCAATTTTGAAGCTTTTATACTGTGAAATTTCCAGCACAGACTCTATTACTGTTGGC GGGCATCCAACCTTTCGTCTCTCTCTACCCTCCAGGACTCCATGATGGATGTCCAAAGTGTTTCATTGGTCC TCGACGTATTGAACACGCCCCTGAAAACCGCCCCTAGGTATCCAAGCGTGGAAGCCGACTTCAGTCAGTTG ATGTGGCGCGATGGAATCGGACCGTTGTGAAGgctagctatagttctagaggtaccgegggeccgggatcgatccaccggtcgccaccatggc ctcctccgaggacgtcatcaaggagttcatgcgcttcaaggtgcgcatggagggctccgtgaacggccacgagttcgagatcgagggegagggcgagggccgcccctac gagggcacccagaccgccaagctgaaggtgaccaagggcggccccctgccettcgectgggacatcctgtccccccagttccagtacggctccaaggtgtacgtgaagca ccccgccgacatccccgactacaagaagctgtccttccccgagggcttcaagtgggagcgegtgatgaacttcgaggacggeggegtggtgaccgtgacccaggactcct ccetgcaggacggctcettcatctacaaggtgaagttcatcggcgtgaacttcccctccgacggccccgtaatgcagaagaagactatgggetgggaggcctccaccgagc gcctgtaccccegcgacggcgtgctgaagggegagatccacaaggecctgaagctgaaggacggeggccactacctggtggagttcaagtccatctacatggecaagaag ccegtgcagctgec ggetactactacgtggactccaagctggacatcacctcccacaacgaggactacaccatcgtggagcagtacgagegegecgagggecgecacca cetgttcctgtagcggccgegactctagatcataatcagccataccacatttgtagaggtttacttgctttaaaaaectcccacacttccccetgaacctgaaacataaaatgaat gcaattgttgttgttaacttgtttattgcagcttataatggttacaaataaagcaatagcatcacaaatttcacaaataaagcattttttcactgcattctagttgtggtttgtccaaactc atcatgtatcttaagcttggegcgec 


\subsubsection{Vector map of the `Tc-rx Intron’ construct}

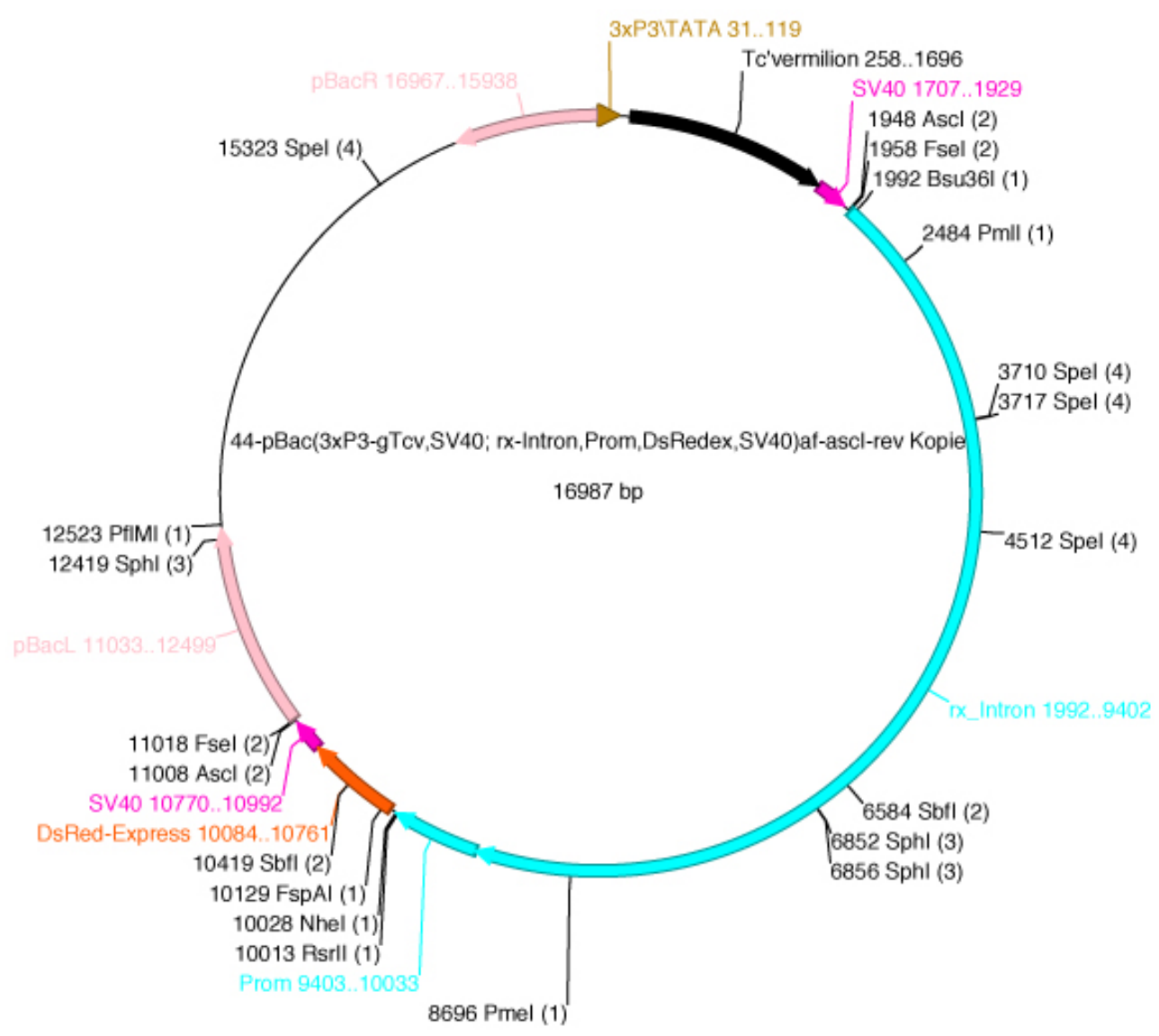

$>T c-r x$ Intron::DsRedEx

ggcgcgcctaggccggccgaattcgaatggccatgggacgtcgacctgaggCACCGTAATTACGCAAAGATAACAGACTCCAGTTACGC CAAAACGAGCAAAATTAATCCAAATTAGAGCCGACTTAACTCGCTATTAATTATGCCAATTATGTGCACGCA ATGTGTGAACTGGGTATAAATTTTTACTTTGTAACGCCTTATTTTTAGTTAAATCTATTGCTTGTACATAAGAT TTAGATTCAACAAACAACGCACTCAAACACGCAATTAAGTCGCCTTCATCCTATTTGTCAATCTCGTAATCC TATCAAATATAATTCCCTGTCAAGTAAGCTGACATCAAATTAGTGCTGCTGTCAATACGGAATAAAGACGGC AAAAACGTCTAAAATCAGAGCTTATTTTTATCTTTGATTTAACAAATAAGCTGATGAATAAAAATAACATCAG CTAACACAAAACTCCAGCTTCAGATGAGGACTGTACTTTAATATTTTTTGTCAAATAAGACTTACCCTGTATA TTTTTTTTCATCACGTGTAAAAATTAGTTTTTTTATTAAAATAATTTTCATTTAAATTTTCAAACAATCAAATAT AAAAATTTGGTTCGTGCTACTTCAAAATATTAAAATACGTGTATGATTTTCCTTAGAGAAGAAAAATAGAGT AAGCCACTTTATTTGCCTTAAAAAATATATTAAACAGAGTAATTAATCTATTTTTTTCAATCAGAAAGCACAA CTGTGAACGATTGTTTCACAAATACCTACTGAAAGCGGGAGACACTCAAGTTAGTTGATAAGTTTAAATTAA ACTTATAAAACTACTTTGTATCTGGTGGTTGTAATGTTAATCAAATGCAAGCTTTATGCTTAGAACGGTTAGT TAAAAGCATTTGGACTGGTCATAGTAAAAGCATTTAGGGAGTGTGGATATTTACCAATCGAGATTTTTTCGG CTCCCGTCCCAAGAAACAATAAATCTGGCTAATGGATTAAGCACTACCGTGACTAAGTTATTTGTTAATTGAT GGGTGTAACTTATCAAATTTTAATTAATTTACTCATTAACATCAACTCAACCACTTATCACGACTTGAAATGG TCGAAACGCACGATTTAGTTAATAATGGTTTTCCAGTGGAAATTTTTAAGAGATTGAAAAATTATTAATCGTA CTTTTCAAGGACACATTGTTAATTGAGAGAGTGTTTTCTTAATTGCTTTATACTTAAAATTATCAATTTATTTA ATTAATTCATTTGTGTAGTTGTATTGAACTGTCGGCTGTTTAATTACCTTCAACTGTTTTAAATTGGTAATTAA GTTGAAGTAGTTATTCATGAGTCGTCTTCTGATAGACACGCCTTCTCTTTTAAACACCATAAAGATGAAGCT TCTGGCTTTCAACTGTGAGAGTCGTAAAAAATTACCGAGAGAACAGCTTCTTAATTAGTAGTAGGTAGGTA GGTGGTATTGGGACTTAATGTTTTATTTTGAAATGAATTAAATCCTACAGCTTCACTCTTTGTTTTTTTTTAAT AAAATGTTTACAATCACTTAGTAATATTCTTAAAAATCTTGCACAATTACTTTTGTTTTGTTTTGCCCCATTTG TTCAGTTCTTGACTACAATTTATTATTTTATGGACAATATACCCAGGTAGGTATAGGTATAGGAATTTTTTAAA TAAAGTCTAATTCTTTATAATCGGCGATTAGCGCATTTTTAAATTTATTTCAGTGTCATAAACTCATTACTAGT CACTAGTTATGGAACAGTAACCATACTGTAGATACAGGAACATAAAAATAAAGCAAAAATCTATAAAAATAC 
ACTGAGTTTTTATAATGGAGTGCATGATTCAGAAGAAATAAACTAGGAAGTCTACATTTTGGTGAATGTTTT TTGCCGACCAAAAGTCAATAATTTATTTGTTACTAATCTGGGAAAACATCTTTTTTAGATGTTTACATATTTCA GATAAATACAAAACAAAGGTCATCATTTTAGACCATTTTTTTTCGATACAAATGGCTTAATTTTTTTATTTTTA CACAGATGTTGGTAAAAAATTACTTTTAAAATACAGAAACTAATTTTTTATTAAAAAAATCAGTAGAAACTA TCACTTTTGGCCTTTTTTCTGTTGTGCTGGAGAACACTATTAAAAGTAAGAAAACAGTCAACAAAATAAATT ACTTTTAGAACTCAATTACCTCAAAATTTTCGTAATAAAAATCAAGAAGGGTTTCTTTGTTTTCAAAAATGC TAAAAATATAAAATACTAGGTACCACATCTTTTCTAGTGTTAAAGAGTCAAGGTGAAAAAAGTGAATTTGCA AATCATTTAAAAATTTCCTGCAGAAGGCAAAAACGACTGCAGTTATTTCCTAAGTCAATGACACCAACTCTT TGGGGACGATTCTAATCTTTATTTTGACCAAATAAGAGCCATAAACTGTCGAGTATTAAGATTTATCATCAAA TTGCATTGTTTGGTCAGTAGTGCCACCTTCTGATTCTTTAATTTATTTCTCAAACTGGCAGTACTAGGTGCAC TAGTGAGACGATAATTTTTTCAGAAATAATTTCAAACTTTGTTTATGAGCAAAGTGTTGGTGCAAAATTTTA GAAAGGTTATTTTGGGTTCATCCAATAAGCCTCAAAAAAAACTGCCTCACCTCAAACACAAAAGACAACA AATTGTCAAAGTACATATAATTTTAAATTTATTATCTTTTAGATAGTAATAGTATGTTTTATGGTAAAAAATTAG GTGTTTTCATCTTCTAAATACCAATTTATGATCTTTTTCCAAATCGACGATATCCTGACTATGCCATATATTTCA CAAAGAAGCAACACAGCTAGAACTACTGTTCTCTTTGTCCTAGTACCGTCATTAAATCAGTTACCTTCTAAA AACATGAATAAATAGGGTGAACAACTGGCCAAGTTTATACAAAAAAAGCGCATTGTTACTTACTCTTTAGAA TAAGTTAATTTTATTAGAAGAATGCAAAAGGTCACCGCTTTATATTCTTCCGAAAATTTCAGCATCATTTTAC TTCTTCTAAATGATTTTACATGAAAATGTAAACTACTAATTATGTAATAATTACTGTTAAACACACACTAATTT ATAGTTTTGTGATAACAATGCAAATGCAATAGTTTGTAAAATTAGCTCAGAACCAGGATCGAAATTGTTTAAT CAAAAGCCGGCGTTTGAAACGTCAATGTGGTACAAACGCAAAGGAAGTTTTCTATTTAACAATCTGAAAGA AAAATTATGCTGTCGTGCTGTTAACGAATTGTACCCAGTTATAATTTTGACAAGTTCTTTTAGCTAATAAACG AAATAAATTTGAATGTAATACGAAATTAACCACAATTCTGCAAACAATGTTAACAAAGCATCTAGACACGAA AAGGCTTCCAGTAAAATGTCCGGTTTGCATGTATATCGCTAGTCCACGCTTCTACCGACTCCGTTTTAGAATC ACAAGCGGTAAAAGGGTAATTACCATCGACAGTCTGTAATATCTAACTCAATTAACGTTTTATGTGCAGTAA AACGGGTACTGGCCATGGAAGGCGGTACAAGCCATCACTGCATTAGCGGCATTTCTAGTTAAATCGAAATA AAAACATCAAGTTAAGTAATCTAAGTGGTGTTCCATTAAACCCAGCGTATCACGGGTGACATGATTATGAAC ACGCGAACCCTGACAGCACAGAATAACTCCTACCTTCACCCACACGAAAATAAACCAAAACACCGGAGCG TATTATTACTACATTAAACGCTGAAATTAGATTTATAAATACGACAAGATGGAAAAGAACATGAAACGCGCC CAATTTCATCGATGGTTTAGATACCTGGTCGAGGCTAGAATAAATTTGGAAATAAAACGAGTCGAAAGTGTC GACTCGGGCGAGATCAGAAGGAAGATATAAGTAGTTGGCTGTCAGACGATTTCTGAGTGCTTGGGTCCGAC TTTATACTTTATTTAATTTGCCAGATCGAAGATAAGCAGGAATTAAAACAAGATATTCACATCTCTGAAACGC GTGTAATCACCATAATCGACTCTGAAATGTCTCTATTTAATTCCACGAACTCAGCTCGGACTTTCAAGTTTAT TATAATCAACTTTTTGTTGTTTGAAGTTAAAACGATTATTCATTTCTCGCCGTCTGAAACTACTTCCAGTAAA TTACTTTTTCTCCGGCGCAATGTAATTAACTATGTTATATAAAACCTCAACGCAGCTACTAATTAGTTGGGTTT ACAAATTAATTATCCTTCAAAAAGTGATTAATTTTTGAACCCGGCCAGAGATTGTCAATCGGTGTAATTTCG GTATTTCGAGATAAAATTAAATTGAATTAAAATAATTAAATTTGTCCACTCGAAAACCTGCTCGGTGGGTGA GTGAATTTTGCACAAAAGCAGATTTTGTTTATTTTGCTCAAGCAGTAAATTAGACCGCTCCAGTGAATTGTT GGGGACCGCTGGCCTCGTGTGCGGCCTCCTGTCGCTTCATAACACGCCTTTAACAAGCCATTGTTTAACAA GAATAGTCTTTTAATTGATGAATTAAGGAAAATTAAGCCGTAGAGCCTGCAGGTGGGATGTTGGGCCACTTA GCGTCGATTGGCAGCACTGATTTTATCGAGCCCATTTCGGCTGAAACCCTGCCGAGCAACATTTGGGTTTTA GGAATTTTCCCGCGAGCGTGTGGGGCAAAACATTAGTAGTTCTTGAGTTAAGCAATTGATTATTTAATTAGG AACATTGATCCGGTCGTTTCGTGCGATCTAATTGCCTTGCATTAATAATAAACACAACAGATTTGCGATAAGC CCGGTAATTGTTAATTGCGTTTTTGCATGCATGCGTCTTTTACACAGGTCTTTAAATGTCCCTTGGTTGTTTA ACATTGTATGCCCCCGTAATCGTGTGCAGTTTCTGCATCGCGTCAAAAAGGCCGTTTATTGCATAATTTGTTA TTTTTCCAAGTTAATTTCGTAAATGTCTGCTGCAATTAAAATTTGCATGTTGGTCGTGTTTTAGCTTCGTCCG AATAATTGCAAATGATTTGTTGAAAGTTTTTCAATGCACACAATGATGGAATTAATTTTGCTGAAACTAAATA AAAACATACAACACAGTCGCTTTGATTATTTTTAATTTCGCCATCATTGTTACAAAAAACTACGGCCTGAAAT TTCCGTTCCTAATTATTCGAATTTTATCGTCCATTCCCATCGGCGTATTTTCCATTTCTTTCTCGCTCACATTAT GTGGCTTTTACATGCAATTAGCGGCAACGTTTCTCGACTGCCGCTTAATAAAATCTCGTGTATGTGCAGTTTT AATTTTTCCAATTTGCATATACGTCAGGTGCGATTAATCAGAGTGTGTTGGGCTTATAATGTAATAGGCAAAG TGCGTTTTTTTAATCATGCAAAATACTAATAAATTTTGTCTATAATAAAAGTCACATTAGATTTGTTTAATGTT ACAAGGGTTGGAATTAAAATGACATATTGCGGACTATAATTAATTGTATATTATTTAAATTAATTCACTTTAAT TGATAACAATTTTTACATAATGCTACATTTCATGATTTATTGCCCCGCAATATAAAACTAATTTCACATACTATT GCAAAACCAATCAACCTCTACTTAGGCAGGCCGGAAGTCTTAACGTGTTATTAGGTTTGCACATTTGTGGTA GTCTTTATAAACAGGATAGGCAGTTTGCAGGTTTTAAACTACATAGTTTTATTTGAATTCATTTGAAGCAATC 
AAATATTAATCTAAAAAAATTATTAAATACTGTTAACAAAGATATTTAAAAATGGTGGATGCGCCGGGGTATT TAAATATGAATATTAAAATTGATTTATTTGCTTCTGTGGGTACTTCCTTTAAACTTACATGCATTTTGATAAAAT GCTTATTATACTTACTACATGAAGGAAATCTTAAAGCAATTTATTTTTAAACAAATATAAATTTGAGTATGAAA ATTCAAATGGAACATGTCTTGATTTTAAATCTACCACAGTTGGTCAGCTTATAAATAAGCCCAGTAGGGTCA CTATTGAAACCAATAAGTGAATATTTTCTCAAGAAAAAATAAACCACGTTCTTGGTCTTATTGCCTTAACCAT GCTTTGAAATCAATACCAAAACTAAAAAAACATATTTTTTTCAAAAATTTTTGACAAGACCTTTGAGCAAAC TAATAAAAAAGACTTTATTGTTTTATTTTGGTTAAAAGTCAAAAAGCAAATTCGGCACTTTTGATAAAAATG TAGATTGGCTTTCAAGACATAGCTTTATAACACAATACTCTTCGAAAGCAAGAACAAGACTAGAAAGCTCA AGACTTTTCGAAACCAAAATCTAGTTTAGGTTTGGTCTTGGGTACACCTCCAATGTACAGAGTTTTCAGGCT TTTGTGTTTAACTATTGTTATTCTACTCATGATATGATACCAAACAGTCTTGATTTTTTTAATTTTTATATGATC CACATCTTTTATATTTCTATATTTTCTGTATGATTTTTTTTGTTTGATCTTATTACATTTGCTGGATGTAGTACTT GTTTCTATTGACGACTTAAATGGAAACAATTTGCCTAACTTCGTTGATAGTAGCCAATTTATCATTATTTATCT TTAACTTCCAAATATTTTTCTTGTTGTTTATAATCGTTTAAACCATTTTTTAACTGAATGCTTTTTTGCCCTAAT TCTATGTCTTGTCTTGGCTTTCATCCAGTTCTATTCAGTTTTCTCTAAGGCATGAACTTTTCGTTGTAGTGTAG GTATCTAGTTTAAATCTTTCTTCATTTATCCAGAGGTATTGTTTCAAAATAACAAATTTAGATGTACCTATCTT TAGAATAGTTATAAACAGTGCATGTTAAAAAAATTTTTCCTTTACATTATACTTCAAGCAAATCACAAAAATG TTTAAGAAATAGGCATAAGTTGTTTGAAATATTGAAATAAAAAAAATGCATTTTTCTGATCGACAATCCCATG TGGGGTCCGTTCTTTGGGGTGATTTTTCGTGCCTGCTTGTAGTTGGTAATCAAGCGTATCAAATTGTCCATTT GAACACATAGTTAATTTCCTAATCGATACAGGATACTGCAATAAATAAATGATTCCGGTAATAACAATTTGGG AGTTTAATAGACTGGTGTTGTTTGAGAACGTGTCGAGATTATGTCTGATAAGAAGGTAATTAATGTGTTTCT GCATTGATAAATAATTGAGGGAGCGTTTGAAAGCGATAGTTGAGGCGATAGTGCGGACATTGCACATTAGGT TATGATATATAATGTGTATCGCGTGTTTGTATTGTATTCTATTAGTTAGGAACATTGTCGGCTTGATTACCGCCC TGggatccTCCTCTAATTATAGCTGAATACAGAGCCGAGTTCTTATACGTAATGGAAAACAAAACTATTACTAAT AATTTGCGTAATATGCTGTGTCAAGTTACATTATTTTAAAAAGTGAACGTTCCCAAAAAATAAGATTTCTTGA GCCAATTTATCGCAATTTAGGTCCAATTTGTCTGACCTACGAATCGATATTTCACCAATTACTCGTATTTCCAG TCCCCAAACCCGCTGTAAAATTAGTTTGAATATCTGAAGTGTGAACGAGGCAACACTTGAGACCCGTTGGT TTCCCAGTAATGGAATAACTGGCAAGCCCAATAACATATCTTATTGGCAGCCCCACTTAAAAACGAGTAGCA ATAGCAACATGTACTACTTAATCCAGATTGTTGACACCAATTTTGAAGCTTTTATACTGTGAAATTTCCAGCA CAGACTCTATTACTGTTGGCGGGCATCCAACCTTTCGTCTCTCTCTACCCTCCAGGACTCCATGATGGATGTC CAAAGTGTTTCATTGGTCCTCGACGTATTGAACACGCCCCTGAAAACCGCCCCTAGGTATCCAAGCGTGGA AGCCGACTTCAGTCAGTTGATGTGGCGCGATGGAATCGGACCGTTGTGAAGgctagctatagttctagaggtaccgegggec cgggatcgatccaccggtcgccaccatggectcctccgaggacgtcatcaaggagttcatgcgcttcaaggtgcgcatggagggctccgtgaacggecacgagttcgagat cgagggcgagggegagggecgcccctacgagggcacccagaccgccaagctgaaggtgaccaagggeggccccetgecettcgectgggacatcctgtccccccagtt ccagtacggctccaaggtgtacgtgaagcaccccgccgacatccccgactacaagaagctgtccttccccgagggcttcaagtgggagcgcgtgatgaacttcgaggacgg cggcgtggtgaccgtgacccaggactcctccetgcaggacggctccttcatctacaaggtgaagttcatcggcgtgaacttcccetccgacggeccegtaatgcagaagaag actatgggctgggaggectccaccgagcgcctgtacccccgcgacggcgtgctgaagggegagatccacaaggccetgaagctgaaggacggeggccactacctggtg gagttcaagtccatctacatggccaagaagcccgtgcagctgeccggctactactacgtggactccaagctggacatcacctcccacaacgaggactacaccatcgtggagc agtacgagcgegecgagggecgccaccacctgttcctgtagcggecgegactctagatcataatcagccataccacatttgtagaggtttacttgctttaaaaaacctcccaca cttccccetgaacctgaaacataaaatgaatgcaattgttgttgttaacttgtttattgcagcttataatggttacaaataaagcaatagcatcacaaatttcacaaataaagcatttttt cactgcattctagttgtggtttgtccaaactcatcatgtatcttaagcttggegcgcc 


\section{Abbreviations}

AL

Antennal Lobe

CB

Central Body

$\mathrm{CC}$

Central Complex

CNS

central nervous system

D

deuterocerebrum

DsRedEx

Discosoma sp. red fluorescent protein Express

dsRNA

double-stranded RNA

gDNA

genomic DNA

HS

heat shock

MB

Mushroom Body

NB

Neuroblast

$\mathrm{P}$

protocerebrum

PB

Protocerebral Bridge

Pe

Pedunculus

PI

pars intercerebralis

RNAi

RNA interference

SB

San Bernardino

StNS

stomatogastric nervous system

$\mathrm{T}$

tritocerebrum

tGFP

turbo green fluorescent protein

TLS

translation start site

TSS

transcription start site

UTR

untranslated region

VW

vermillion white

WT

wild type 


\section{Curriculum vitae}

Name: $\quad$ Nikolaus Dieter Bernhard Koniszewski

Date of birth: $\quad$ September $20^{\text {th }} 1980$

Place of birth: $\quad$ Erlangen, Germany

Nationality: German

Current address: Bertheaustraße 25, App. 73, 37075 Göttingen

E-mail: nikolaus.koniszewski@gmx.de

\section{Phd studies}

since 2007:

Georg-August-University Göttingen

$\mathrm{PhD}$ student under the supervision of

Juniorprofessor Dr. Gregor Bucher at the

Department of Developmental Biology

Title of thesis: Functional analysis of embryonic brain development in

Tribolium castaneum

\section{University studies}

2007:

Diploma in Biology of the Friedrich-Alexander-University

Erlangen/Nürnberg

2006-2007:

Friedrich-Alexander-University Erlangen/Nürnberg

Diploma thesis under the supervision of

Professor Dr. Martin Klingler at the

Department of Developmental Biology

Title of the thesis: Das Terminale System in Tribolium castaneum

2004-2006: Main course in Biology at the Friedrich-Alexander University

Erlangen/Nürnberg

2002-2004: $\quad$ Basic studies in Biology at the Friedrich-Alexander University

Erlangen/Nürnberg

\section{Civil service:}

2001-2002:

Civil service at the "Klinikum Forchheim der Vereinigten Pfründnerstiftung"

\section{High School:}

2001:

Abitur at the Ehrenbürg Gymansium Forchheim, Germany 


\section{Scientific memberships}

- Member of the Göttingen Center for Molecular Biosciences (GZMB)

- Member of the Göttingen Center for Molecular Physiology of the Brain (CMPB)

\section{Publications}

- Schinko, J., Posnien, N., Kittelmann, S., Koniszewski, N., and Bucher, G. (2009). Single and double whole-mount in situ hybridization in red flour beetle (Tribolium) embryos. Cold Spring Harb Protoc 2009

- Posnien, N., Koniszewski, N., and Bucher, G. (2011). Insect Tc-six4 marks a unit with similarity to vertebrate placodes. Dev Biol 350, 208-216

\section{Public presentations}

- Koniszewski N., Bucher G.

What makes neuroblasts different from each other? (Poster and Oral presentation)

3. International Tribolium Meeting, Göttingen (Germany), September 6, 2007

- Koniszewski N., Hein H., Bucher G.

Tools for analyzing neural stem cell identity in the larval brain of Tribolium castaneum (Poster)

From Neurogenesis to Synaptogenesis, Göttingen (Germany); September 25 - 26, 2008

- $\quad$ Koniszewski N., Hein H., Bucher G.

Tools for analyzing neural stem cell identity in the larval brain of Tribolium castaneum (Poster)

The $8^{\text {th }}$ Meeting of the German Neuroscience Society, Göttingen (Germany), July 25 - 29, 2009

- Koniszewski N., Bucher G.

Transgenic imaging lines to analyze Tribolium brain patterning (Poster and Oral presentation)

Cologne's Regional Tribolium Meeting, Cologne (Germany), July 27 - 29, 2009

- Koniszewski N., Bucher G.

Transgenic imaging lines to analyze Tribolium brain patterning (Oral presentation) $20^{\text {th }}$ Neuro Do-Wo Würzburg '09, Würzburg (Germany), July 30 - 31, 2009

- Koniszewski N., Bucher G.

Tc-six3 is required for central complex development (Oral presentation)

International Tribolium Meeting Paris, Paris (France), July 5 - 6, 2010 